Cochrane Database of Systematic Reviews

\title{
Olanzapine for the prevention and treatment of cancer-related nausea and vomiting in adults (Review)
}

Sutherland A, Naessens K, Plugge E, Ware L, Head K, Burton MJ, Wee B

Sutherland A, Naessens K, Plugge E, Ware L, Head K, Burton MJ, Wee B.

Olanzapine for the prevention and treatment of cancer-related nausea and vomiting in adults.

Cochrane Database of Systematic Reviews 2018, Issue 9. Art. No.: CD012555.

DOI: 10.1002/14651858.CD012555.pub2.

www.cochranelibrary.com 
TABLE OF CONTENTS

HEADER

ABSTRACT

PLAIN LANGUAGE SUMMARY

SUMMARY OF FINDINGS

BACKGROUND

OBJECTIVES

METHODS

Figure 1.

RESULTS

Figure 2.

Figure 3.

DISCUSSION

AUTHORS' CONCLUSIONS

ACKNOWLEDGEMENTS

REFERENCES

CHARACTERISTICS OF STUDIES

DATA AND ANALYSES

Analysis 1.1. Comparison 1 Olanzapine vs placebo/no treatment, Outcome 1 No nausea or vomiting over trial period. ............

Analysis 1.2. Comparison 1 Olanzapine vs placebo/no treatment, Outcome 2 Serious adverse events.

Analysis 1.3. Comparison 1 Olanzapine vs placebo/no treatment, Outcome 3 Participant preference - wish to use drug in next treatment.

Analysis 1.4. Comparison 1 Olanzapine vs placebo/no treatment, Outcome 4 Other adverse events.

Analysis 1.5. Comparison 1 Olanzapine vs placebo/no treatment, Outcome 5 Somnolence/fatigue.

Analysis 1.6. Comparison 1 Olanzapine vs placebo/no treatment, Outcome 6 Withdrawals due to all causes.

Analysis 1.7. Comparison 1 Olanzapine vs placebo/no treatment, Outcome 7 Withdrawals due to lack of efficacy.

Analysis 1.8. Comparison 1 Olanzapine vs placebo/no treatment, Outcome 8 Withdrawals due to adverse events.

Analysis 1.9. Comparison 1 Olanzapine vs placebo/no treatment, Outcome 9 Breakthrough nausea/vomiting requiring antiemetics over trial period.

Analysis 1.10. Comparison 1 Olanzapine vs placebo/no treatment, Outcome 10 No nausea over trial period.

Analysis 1.11. Comparison 1 Olanzapine vs placebo/no treatment, Outcome 11 No vomiting over trial period.

Analysis 1.12. Comparison 1 Olanzapine vs placebo/no treatment, Outcome 12 No acute nausea (within $24 \mathrm{~h}$ of chemotherapy).

Analysis 1.13. Comparison 1 Olanzapine vs placebo/no treatment, Outcome 13 No acute vomiting (within $24 \mathrm{~h}$ of chemotherapy).

Analysis 1.14. Comparison 1 Olanzapine vs placebo/no treatment, Outcome 14 No acute nausea or vomiting (within $24 \mathrm{~h}$ of chemotherapy).

Analysis 1.15. Comparison 1 Olanzapine vs placebo/no treatment, Outcome 15 No delayed nausea (1-5 days after chemotherapy).

Analysis 1.16. Comparison 1 Olanzapine vs placebo/no treatment, Outcome 16 No delayed vomiting (1-5 days after chemotherapy).

Analysis 1.17. Comparison 1 Olanzapine vs placebo/no treatment, Outcome 17 No delayed nausea or vomiting (1-5 days after chemotherapy).

Analysis 2.1. Comparison 2 Olanzapine vs NK1 antagonist, Outcome 1 Serious adverse events.

Analysis 2.2. Comparison 2 Olanzapine vs NK1 antagonist, Outcome 2 Somnolence/fatigue.

Analysis 2.3. Comparison 2 Olanzapine vs NK1 antagonist, Outcome 3 Withdrawals due to all causes.

Analysis 2.4. Comparison 2 Olanzapine vs NK1 antagonist, Outcome 4 Withdrawals due to lack of efficacy.

Analysis 2.5. Comparison 2 Olanzapine vs NK1 antagonist, Outcome 5 Withdrawals due to adverse events.

Analysis 2.6. Comparison 2 Olanzapine vs NK1 antagonist, Outcome 6 Withdrawals due to death.

Analysis 2.7. Comparison 2 Olanzapine vs NK1 antagonist, Outcome 7 No nausea over trial period.

Analysis 2.8. Comparison 2 Olanzapine vs NK1 antagonist, Outcome 8 No acute nausea (within $24 \mathrm{~h}$ of chemotherapy). ......... Analysis 2.9. Comparison 2 Olanzapine vs NK1 antagonist, Outcome 9 No delayed nausea (1-5 days after chemotherapy). ..... Analysis 3.1. Comparison 3 Olanzapine vs prokinetic, Outcome 1 Serious adverse events. 
Analysis 3.2. Comparison 3 Olanzapine vs prokinetic, Outcome 2 Withdrawals due to all causes.

Analysis 3.3. Comparison 3 Olanzapine vs prokinetic, Outcome 3 Withdrawals due to lack of efficacy.

Analysis 3.4. Comparison 3 Olanzapine vs prokinetic, Outcome 4 Withdrawals due to adverse events.

Analysis 3.5. Comparison 3 Olanzapine vs prokinetic, Outcome 5 Withdrawals due to death.

Analysis 3.6. Comparison 3 Olanzapine vs prokinetic, Outcome 6 No nausea over trial period.

Analysis 3.7. Comparison 3 Olanzapine vs prokinetic, Outcome 7 No vomiting over trial period.

Analysis 4.1. Comparison 4 Olanzapine vs 5-HT3 antagonist, Outcome 1 Serious adverse events.

Analysis 4.2. Comparison 4 Olanzapine vs 5-HT3 antagonist, Outcome $250 \%$ improvement in nausea at $24 \mathrm{~h}$ on a validated scale.

Analysis 4.3. Comparison 4 Olanzapine vs $5-\mathrm{HT} 3$ antagonist, Outcome $350 \%$ improvement in nausea at $48 \mathrm{~h}$ on a validated scale.

Analysis 4.4. Comparison 4 Olanzapine vs 5-HT3 antagonist, Outcome 4 Withdrawals due to all causes.

Analysis 4.5. Comparison 4 Olanzapine vs 5-HT3 antagonist, Outcome 5 Withdrawals due to lack of efficacy.

Analysis 4.6. Comparison 4 Olanzapine vs 5-HT3 antagonist, Outcome 6 Withdrawals due to adverse events.

Analysis 4.7. Comparison 4 Olanzapine vs 5-HT3 antagonist, Outcome 7 Withdrawals due to death.

Analysis 5.1. Comparison 5 Olanzapine vs dexamethasone, Outcome 1 Serious adverse events.

Analysis 5.2. Comparison 5 Olanzapine vs dexamethasone, Outcome 2 Withdrawals due to all causes.

Analysis 5.3. Comparison 5 Olanzapine vs dexamethasone, Outcome 3 No nausea over trial period.

Analysis 5.4. Comparison 5 Olanzapine vs dexamethasone, Outcome 4 No vomiting over trial period.

Analysis 5.5. Comparison 5 Olanzapine vs dexamethasone, Outcome 5 No acute nausea (within $24 \mathrm{~h}$ of chemotherapy). ......... Analysis 5.6. Comparison 5 Olanzapine vs dexamethasone, Outcome 6 No acute vomiting (within $24 \mathrm{~h}$ of chemotherapy). ..... Analysis 5.7. Comparison 5 Olanzapine vs dexamethasone, Outcome 7 No delayed nausea (1-5 days after chemotherapy). .... Analysis 5.8. Comparison 5 Olanzapine vs dexamethasone, Outcome 8 No delayed vomiting (1-5 days after chemotherapy). .... Analysis 6.1. Comparison 6 Subgroup (dose): olanzapine vs placebo/no treatment, Outcome 1 No nausea or vomiting over trial period.

Analysis 6.2. Comparison 6 Subgroup (dose): olanzapine vs placebo/no treatment, Outcome 2 Serious adverse events. .......... 
[Intervention Review]

\section{Olanzapine for the prevention and treatment of cancer-related nausea and vomiting in adults}

Anna Sutherland 1,2 , Katrien Naessens ${ }^{3}$, Emma Plugge ${ }^{4}$, Lynda Ware ${ }^{4}$, Karen Head 5 , Martin J Burton ${ }^{4}$, Bee Wee 6

1Katharine House Hospice, Banbury, UK. 2Cochrane Fellowship, Cochrane UK, Oxford, UK. ${ }^{3}$ Palliative Care, Oxford Radcliffe Trust/Health Education Thames Valley, Oxford, UK. ${ }^{4}$ Cochrane UK, Oxford, UK. ${ }^{5}$ Cochrane ENT, Nuffield Department of Surgical Sciences, University of Oxford, Oxford, UK. ${ }^{6}$ Nuffield Department of Medicine and Sir Michael Sobell House, Churchill Hospital, Oxford, UK

Contact address: Anna Sutherland, Katharine House Hospice, Mandeville Road, Aynho Road, Adderbury, Banbury, OX17 3NL, UK. annasutherland@doctors.org.uk.

Editorial group: Cochrane Pain, Palliative and Supportive Care Group. Publication status and date: New, published in Issue 9, 2018.

Citation: Sutherland A, Naessens K, Plugge E, Ware L, Head K, Burton MJ, Wee B. Olanzapine for the prevention and treatment of cancer-related nausea and vomiting in adults. Cochrane Database of Systematic Reviews 2018, Issue 9. Art. No.: CD012555. DOI: 10.1002/14651858.CD012555.pub2.

Copyright ( 2018 The Cochrane Collaboration. Published by John Wiley \& Sons, Ltd.

\section{A B S T R A C T}

\section{Background}

Olanzapine as an antiemetic represents a new use of an antipsychotic drug. People with cancer may experience nausea and vomiting whilst receiving chemotherapy or radiotherapy, or whilst in the palliative phase of illness.

\section{Objectives}

To assess the efficacy and safety of olanzapine when used as an antiemetic in the prevention and treatment of nausea and vomiting related to cancer in adults.

\section{Search methods}

We searched CENTRAL, MEDLINE and Embase for published data on 20th September 2017, as well as ClinicalTrials.gov and World Health Organization International Clinical Trials Registry Platform for unpublished trials. We checked reference lists, and contacted experts in the field and study authors.

\section{Selection criteria}

We included randomised controlled trials (RCTs) of olanzapine versus any comparator with or without adjunct therapies for the prevention or treatment, or both, of nausea or vomiting in people with cancer aged 18 years or older, in any setting, of any duration, with at least 10 participants per treatment arm.

\section{Data collection and analysis}

We used standard Cochrane methodology. We used GRADE to assess quality of evidence for each main outcome. We extracted data for absence of nausea or vomiting and frequency of serious adverse events as primary outcomes. We extracted data for patient perception of treatment, other adverse events, somnolence and fatigue, attrition, nausea or vomiting severity, breakthrough nausea and vomiting, rescue antiemetic use, and nausea and vomiting as secondary outcomes at specified time points.

\section{Main results}

We included 14 RCTs (1917 participants) from high-, middle- and low-income countries, representing over 24 different cancers. Thirteen studies were in chemotherapy-induced nausea and vomiting. Oral olanzapine was administered during highly emetogenic (HEC) or 
moderately emetogenic (MEC) chemotherapy (12 studies); chemoradiotherapy (one study); or palliation (one study). Eight studies await classification and 13 are ongoing.

The main comparison was olanzapine versus placebo/no treatment. Other comparisons were olanzapine versus NK1 antagonist, prokinetic, 5-HT3 antagonist or dexamethasone.

We assessed all but one study as having one or more domains that were at high risk of bias. Eight RCTs with fewer than 50 participants per treatment arm, and 10 RCTs with issues related to blinding, were at high risk of bias. We downgraded GRADE assessments due to imprecision, inconsistency and study limitations.

\section{Olanzapine versus placebo/no treatment}

\section{Primary outcomes}

Olanzapine probably doubles the likelihood of no nausea or vomiting during chemotherapy from $25 \%$ to $50 \%$ (risk ratio (RR) $1.98,95 \%$ confidence interval (CI) 1.59 to 2.47; 561 participants; 3 studies; solid tumours; HEC or MEC therapy; moderate-quality evidence) when added to standard therapy. Number needed to treat for additional beneficial outcome (NNTB) was $5(95 \% \mathrm{Cl} 3.3-6.6)$.

It is uncertain if olanzapine increases the risk of serious adverse events (absolute risk difference $0.7 \%$ more, $95 \% \mathrm{Cl} 0.2$ to 5.2 ) (RR 2.46, $95 \% \mathrm{Cl} 0.48$ to $12.55 ; 7$ studies, 889 participants, low-quality evidence).

\section{Secondary outcomes}

Four studies reported patient perception of treatment. One study (48 participants) reported no difference in patient preference. Four reported quality of life but data were insufficient for meta-analysis.

Olanzapine may increase other adverse events (RR 1.71,95\% Cl 0.99 to 2.96; 332 participants; 4 studies; low-quality evidence) and probably increases somnolence and fatigue compared to no treatment or placebo ( $\mathrm{RR} 2.33,95 \% \mathrm{Cl} 1.30$ to 4.18 ; anticipated absolute risk $8.2 \%$ more, $95 \% \mathrm{Cl} 1.9$ to 18.8 ; 464 participants; 5 studies; moderate-quality evidence). Olanzapine probably does not affect all-cause attrition (RR 0.99 , $95 \% \mathrm{Cl} 0.57$ to $1.73 ; 943$ participants; 8 studies; $\left.\right|^{2}=0 \%$ ). We are uncertain if olanzapine increases attrition due to adverse events (RR 3.00, $95 \% \mathrm{Cl} 0.13$ to $70.16 ; 422$ participants; 6 studies). No participants withdrew due to lack of efficacy.

We are uncertain if olanzapine reduces breakthrough nausea and vomiting (RR $0.38,95 \% \mathrm{Cl} 0.10$ to $1.47 ; 501$ participants; 2 studies; $\mathrm{I}^{2}=$ $54 \%$ ) compared to placebo or no treatment. No studies reported $50 \%$ reduction in severity of nausea or vomiting, use of rescue antiemetics, or attrition.

We are uncertain of olanzapine's efficacy in reducing acute nausea or vomiting. Olanzapine probably reduces delayed nausea (RR 1.71, $95 \% \mathrm{Cl} 1.40$ to 2.09 ; 585 participants; 3 studies) and vomiting (RR 1.28, 95\% Cl 1.14 to 1.42; 702 participants; 5 studies).

\section{Subgroup analysis: $5 \mathrm{mg}$ versus $10 \mathrm{mg}$}

Planned subgroup analyses found that it is unclear if $5 \mathrm{mg}$ is as effective an antiemetic as $10 \mathrm{mg}$. There is insufficient evidence to exclude the possibility that $5 \mathrm{mg}$ may confer a lower risk of somnolence and fatigue than $10 \mathrm{mg}$.

\section{Other comparisons}

One study (20 participants) compared olanzapine versus NK1 antagonists. We observed no difference in any reported outcomes.

One study (112 participants) compared olanzapine versus a prokinetic (metoclopramide), reporting that olanzapine may increase freedom from overall nausea (RR 2.95, 95\% Cl 1.73 to 5.02 ) and overall vomiting (RR 3.03, 95\% Cl 1.78 to 5.14).

One study (62 participants) examined olanzapine versus 5-HT3 antagonists, reporting olanzapine may increase the likelihood of 50\% or greater reduction in nausea or vomiting at 48 hours (RR $1.82,95 \% \mathrm{Cl} 1.11$ to 2.97 ) and 24 hours (RR $1.36,95 \% \mathrm{Cl} 0.80$ to 2.34 ).

One study (229 participants) compared olanzapine versus dexamethasone, reporting that olanzapine may reduce overall nausea (RR 1.73, $95 \% \mathrm{Cl} 1.37$ to 2.18 ), overall vomiting ( $\mathrm{RR} 1.27,95 \% \mathrm{Cl} 1.10$ to 1.48 ), delayed nausea ( $\mathrm{RR} 1.66,95 \% \mathrm{Cl} 1.33$ to 2.08 ) and delayed vomiting (RR $1.25,95 \%$ Cl 1.07 to 1.45$)$.

\section{Authors' conclusions}

There is moderate-quality evidence that oral olanzapine probably increases the likelihood of not being nauseous or vomiting during chemotherapy from $25 \%$ to $50 \%$ in adults with solid tumours, in addition to standard therapy, compared to placebo or no treatment. There is uncertainty whether it increases serious adverse events. It may increase the likelihood of other adverse events, probably increasing somnolence and fatigue. There is uncertainty about relative benefits and harms of $5 \mathrm{mg}$ versus $10 \mathrm{mg}$. 
We identified only RCTs describing oral administration. The findings of this review cannot be extrapolated to provide evidence about the efficacy and safety of any injectable form (intravenous, intramuscular or subcutaneous) of olanzapine.

\section{PLAIN LANGUAGE SUMMARY}

\section{Olanzapine for the prevention and treatment of cancer-related nausea and vomiting in adults}

\section{Background}

Olanzapine has been studied to see if it might work as an antisickness (antiemetic) medication and if it is safe. People with cancer may commonly experience distressing nausea and vomiting, despite the current medications available, before, during, and after chemotherapy or radiotherapy, and during a palliative phase of illness (when the aim of treatment is symptom relief rather than cure). Some people still experience problematic nausea and vomiting with chemotherapy even when they are given standard antisickness medication.

Recently research studies have focused on preventing and treating chemotherapy-induced nausea and vomiting.

\section{Review question}

We investigated the benefits and harms of using olanzapine for preventing and treating nausea and vomiting in adults with cancer.

\section{Search date}

We searched for studies in September 2017.

\section{Study characteristics}

We included 14 randomised controlled trials (RCTs) because they provide the most reliable evidence, with 1917 participants in total, from all around the world that investigated the use of oral olanzapine in treating or preventing nausea and vomiting.

All the included studies used olanzapine in combination with other medications, usually antiemetics (antisickness medications). Nine studies compared olanzapine to placebo (a substance with no therapeutic effect) or no treatment. Other studies compared olanzapine to other antiemetics.

\section{Were participants receiving anticancer treatments?}

Thirteen RCTs included participants receiving chemotherapy. Chemotherapy is graded according to how likely it is to provoke nausea and vomiting (i.e. how emetogenic it is). In six RCTs participants received highly emetogenic chemotherapy (HEC) or moderately emetogenic chemotherapy (MEC). In six RCTs participants only received HEC. One RCT did not state whether participants received HEC or MEC.

No RCTs included participants receiving radiotherapy alone. One trial included participants who were receiving both chemotherapy and radiotherapy treatment for their cancer. One trial included participants who were not receiving either chemotherapy or radiotherapy.

\section{Study funding sources}

No included RCTs reported receiving funds from pharmaceutical companies. Five studies stated that they had received funding from cancer foundations, endowments, or universities. Nine studies made no declaration regarding funding.

\section{Key results}

Fifty percent of people who received olanzapine as well as standard treatment probably would not be nauseous or vomit during chemotherapy compared to just $25 \%$ of those who received standard treatment. Olanzapine probably makes unwanted sleepiness more likely. We are uncertain if using $5 \mathrm{mg}$ olanzapine a day instead of $10 \mathrm{mg}$ olanzapine a day reduces the likelihood of being sleepy without reducing the antisickness benefit. We are not certain about the risk of experiencing other side effects or serious side effects, so it is important to be aware that these might happen. There was some suggestion that people who take olanzapine with standard treatment might have an improved quality of life compared to those who used standard treatment alone, but we were very uncertain of this because we were unable to analyse the data. There was not enough evidence to say whether people prefer to use olanzapine compared to not taking it.

There is not enough evidence to say whether olanzapine is as good as, worse or better than other antisickness medications currently in use.

\section{Quality of the evidence}

We rated the quality of the evidence from studies using four levels: very low, low, moderate, or high. 'Very low-quality evidence' means that we are very uncertain about the results. 'High-quality evidence' means that we are very confident in the results. We found moderatequality evidence that olanzapine reduces overall nausea and vomiting but that it also increases unwanted sleepiness when compared to 
placebo or no treatment. There was moderate-quality evidence for patient preference and low-quality evidence for adverse events. The remaining evidence was of low- or very-low quality.

\section{Implications of the review}

Olanzapine is probably an effective antisickness medication. We are unsure which dose is best to use, $5 \mathrm{mg}$ or $10 \mathrm{mg}$, or if $2.5 \mathrm{mg}$ might work just as well. However, this review only found information about giving olanzapine by mouth and did not find any about injecting it. More research is needed to inform practice. 
SUMMARY OF FINDINGS

Summary of findings for the main comparison. Olanzapine compared to placebo/no treatment for the prevention and treatment of cancer-related nausea and vomiting in adults

Olanzapine compared to placebo/no treatment for the prevention and treatment of cancer-related nausea and vomiting in adults

Population: adults with cancer

Setting: hospital or medical institutions; either inpatient or not stated if inpatient or outpatient

Intervention: olanzapine

Comparison: placebo/no treatment

\begin{tabular}{|c|c|c|c|c|c|c|}
\hline \multirow[t]{2}{*}{ Outcomes } & \multirow{2}{*}{$\begin{array}{l}\text { Relative effect } \\
(95 \% \mathrm{CI})\end{array}$} & \multicolumn{3}{|c|}{ Anticipated absolute effects ${ }^{\star}(95 \% \mathrm{Cl})$} & \multirow{2}{*}{$\begin{array}{l}\text { Certainty of } \\
\text { the evidence } \\
\text { (GRADE) }\end{array}$} & \multirow[t]{2}{*}{ Results } \\
\hline & & $\begin{array}{l}\text { Without olan- } \\
\text { zapine }\end{array}$ & $\begin{array}{l}\text { With olanzap- } \\
\text { ine }\end{array}$ & Difference & & \\
\hline \multirow{2}{*}{$\begin{array}{l}\text { No nausea or } \\
\text { vomiting over } \\
\text { trial period }\end{array}$} & \multirow{2}{*}{$\begin{array}{l}\text { RR } 1.98 \text { (1.59 to } \\
2.47 \text { ) }\end{array}$} & \multicolumn{3}{|c|}{ Study population: 561 participants (3 RCTs) } & \multirow{2}{*}{$\begin{array}{l}\oplus \oplus \oplus \ominus \\
\text { Moderate } 1\end{array}$} & \multirow{2}{*}{$\begin{array}{l}\text { Olanzapine probably improves freedom from nau- } \\
\text { sea or vomiting over the trial period when compared } \\
\text { to placebo or no intervention if used in conjunction } \\
\text { with standard therapy } \\
\text { (OR } 5.48 \text { (95\% Cl } 1.35 \text { to } 22.20 \text { ) } I^{2}=85 \% \text { ). }\end{array}$} \\
\hline & & $25.1 \%$ & $\begin{array}{l}49.7 \% \\
(39.9 \text { to } 62.0)\end{array}$ & $\begin{array}{l}24.6 \% \text { more } \\
(14.8 \text { more to } 36.9 \\
\text { more) }\end{array}$ & & \\
\hline \multirow{3}{*}{$\begin{array}{l}\text { Serious ad- } \\
\text { verse events }\end{array}$} & \multirow{3}{*}{$\begin{array}{l}\text { RR } 2.46 \\
\text { (0.48 to } 12.55)\end{array}$} & \multicolumn{3}{|c|}{ Study population: 889 participants (7 RCTs) } & \multirow{3}{*}{$\begin{array}{l}\oplus \oplus \ominus \ominus \\
\text { Low }^{2}\end{array}$} & \multirow{3}{*}{$\begin{array}{l}\text { It is uncertain if olanzapine increases the risk of se- } \\
\text { rious adverse events when compared to placebo or } \\
\text { no intervention if used in conjunction with standard } \\
\text { therapy. Six RCTs reported no serious adverse events } \\
\text { in either arm } \\
\text { (OR } 2.50,95 \% \mathrm{Cl} 0.48 \text { to } 13.04 ;\left.\right|^{2}=0 \% \text { ). }\end{array}$} \\
\hline & & $0.5 \%$ & $\begin{array}{l}1.1 \% \\
(0.2 \text { to } 5.7)\end{array}$ & $\begin{array}{l}0.7 \% \text { more } \\
(0.2 \text { fewer to } 5.2 \\
\text { more })\end{array}$ & & \\
\hline & & & & & & \\
\hline
\end{tabular}

\begin{tabular}{|c|c|}
\hline Participant & RR 1.43 \\
\hline
\end{tabular}

RR 1.43

Study population: 48 participants (1 RCT)

Moderate 3

preference -

(0.97 to 2.09)

$58.3 \%$

$83.4 \%$

$25.1 \%$ more

drug in next

(56.6 to 100.0$)$

(1.8 fewer to 63.6

treatment

more)

when compared to placebo or no intervention if used in conjunction with standard therapy

(OR 3.57, 95\% Cl 0.93 to 13.72 ).

Quality of life Study population: 258 participants (4 RCTs)

Mizukami 2014: "[olanzapine] group experienced a better QOL than the control group, as reported on the Functional Living Index-Emesis questionnaire $(P<0.0004)$ ".

It is unclear whether participants who used olanzapine had an improved quality of life compared to placebo or no intervention, if used with standard therapy due to inter-study heterogeneity in scales used. 
Mukhopadhyay 2016: global health status improved in the olanzapine group using the EORTC

QLQ C30. Control group's global health status deteriorated from $10.08 \pm 0.43$ before treatment

to $8.70 \pm 0.44 \mathrm{P}<0.0001$ after chemotherapy. This decline was not seen in the olanzapine group

(before $9.48 \pm 0.48$ and after treatment $9.60 \pm 0.47 ; \mathrm{P}=0.537$ ).

Navari 2010b 23/39 participants in the megestrol plus olanzapine arm had an improved QOL at 4 and 8 weeks compared to $5 / 37$ who received megestrol alone on the "Functional Assessment of Cancer Therapy-General (version 3)".

Nikbakhsh 2016: "no significant difference was observed between the case and control groups about the patients QOL in ... total QOL score $(\mathrm{P}>0.05)$ " using the WHO-QOL-BREF

\begin{tabular}{|c|c|c|c|c|c|c|}
\hline $\begin{array}{l}\text { Patient Global } \\
\text { Impression of } \\
\text { Change }\end{array}$ & - & - & - & - & - & Outcome was not measured or not reported. \\
\hline \multirow{2}{*}{$\begin{array}{l}\text { Other adverse } \\
\text { events }\end{array}$} & \multirow{2}{*}{$\begin{array}{l}\text { RR } 1.71 \\
\text { (0.99 to } 2.96 \text { ) }\end{array}$} & \multicolumn{3}{|c|}{ Study population: 332 participants (4 RCTs) } & \multirow{2}{*}{$\begin{array}{l}\oplus \oplus \odot \odot \\
\text { Low }^{4}\end{array}$} & \multirow{2}{*}{$\begin{array}{l}\text { Olanzapine may lead to more adverse events when } \\
\text { compared to placebo or no treatment if used in con- } \\
\text { junction with standard therapy } \\
\text { (OR } 2.05,95 \% \mathrm{Cl} 0.99 \text { to } 4.22 ; \mathrm{I}^{2}=0 \% \text { ). }\end{array}$} \\
\hline & & $8.4 \%$ & $\begin{array}{l}14.4 \% \\
(8.3 \text { to } 25.0)\end{array}$ & $\begin{array}{l}6.0 \% \text { more } \\
(0.1 \text { fewer to } 16.5 \\
\text { more) }\end{array}$ & & \\
\hline \multirow{2}{*}{$\begin{array}{l}\text { Somno- } \\
\text { lence/fatigue }\end{array}$} & \multirow{2}{*}{$\begin{array}{l}\text { RR } 2.33 \\
\text { (1.30 to } 4.18)\end{array}$} & \multicolumn{3}{|c|}{ Study population: 464 participants (5 RCTs) } & \multirow{2}{*}{$\begin{array}{l}\oplus \oplus \oplus \ominus \\
\text { Moderate }^{5}\end{array}$} & \multirow{2}{*}{$\begin{array}{l}\text { Olanzapine probably leads to increased risk of som- } \\
\text { nolence or fatigue when compared to placebo or no } \\
\text { intervention if used in conjunction with standard } \\
\text { therapy } \\
\text { (OR } 2.84,95 \% \mathrm{Cl} 1.39 \text { to } 5.78 ;\left.\right|^{2}=0 \% \text { ). }\end{array}$} \\
\hline & & $5.2 \%$ & $\begin{array}{l}13.4 \% \\
(7.0 \text { to } 24.0)\end{array}$ & $\begin{array}{l}8.2 \% \text { more } \\
(1.9 \text { more to } 18.8 \\
\text { more })\end{array}$ & & \\
\hline
\end{tabular}

*The risk in the intervention group (and its $95 \%$ confidence interval) is based on the assumed risk in the comparison group and the relative effect of the intervention (and its $95 \% \mathrm{Cl})$.

CI: confidence interval; EORTC QLQ-C30: European Organisation for Research and Treatment of Cancer quality of life questionnaire; QOL: quality of life; RCT: randomised controlled trial; RR: risk ratio; OR: odds ratio; WHO-QOL-BREF: World Health Organization Quality of Life-BREF

\section{GRADE Working Group grades of evidence}

High certainty: we are very confident that the true effect lies close to that of the estimate of the effect.

Moderate certainty: we are moderately confident in the effect estimate: the true effect is likely to be close to the estimate of the effect, but there is a possibility that it is substantially different.

Low certainty: our confidence in the effect estimate is limited: the true effect may be substantially different from the estimate of the effect.

Very low certainty: we have very little confidence in the effect estimate: the true effect is likely to be substantially different from the estimate of effect.

1Downgraded to moderate quality due to inconsistency $(-1)$. The risk of nausea and vomiting in the control group varied from $19 \%$ to $40 \%$. This variation was probably due to the characteristics of the trials being different (such as type of cancer, chemotherapy treatment given, dose of olanzapine etc). We observed statistical heterogeneity when using odds ratios $\left(1^{2}=85 \%\right)$. 


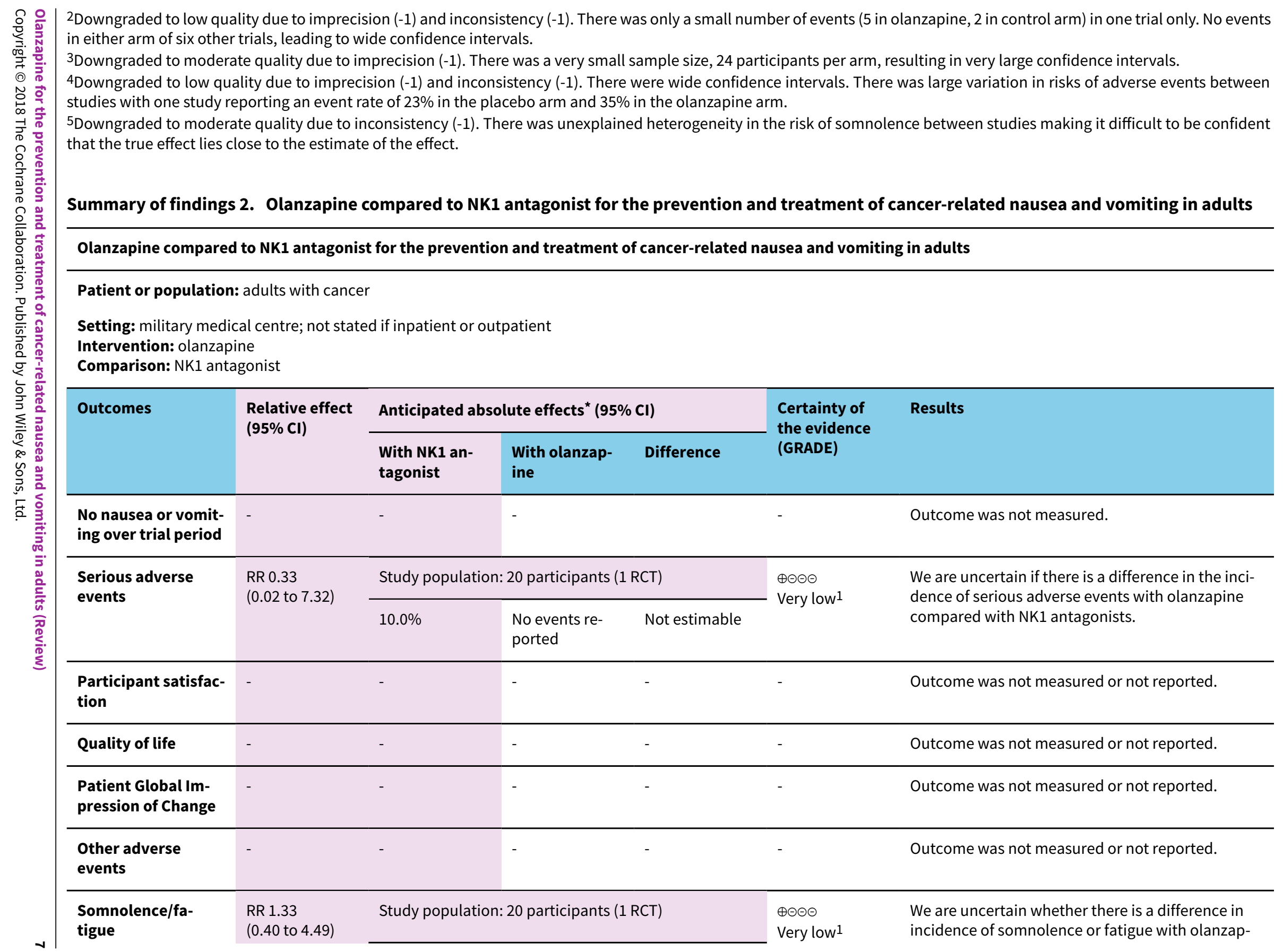




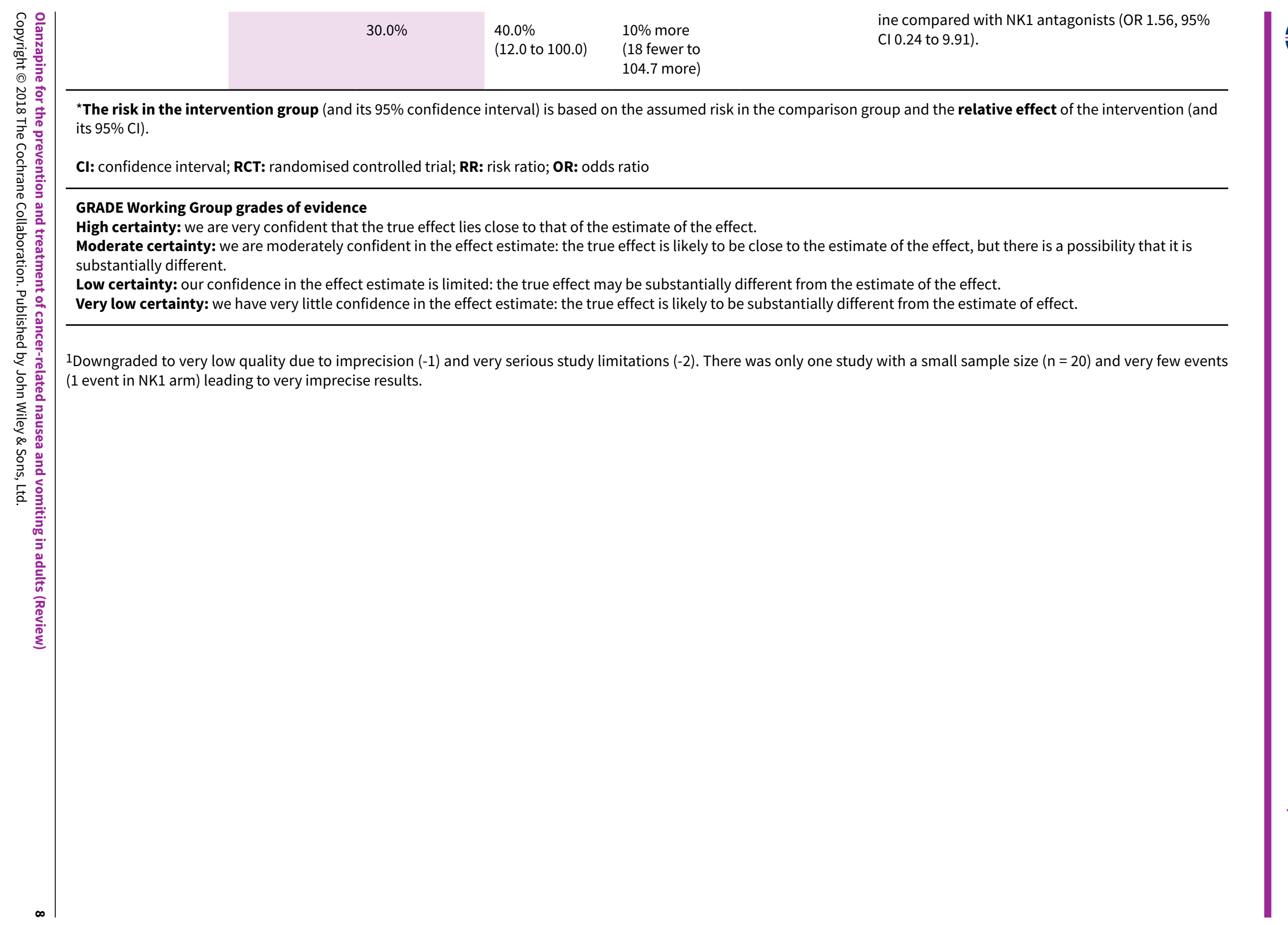




\section{B A C K G R O U N D}

This protocol is based on templates from both Cochrane Pain, Palliative and Supportive Care (PaPaS) and Cochrane Ear, Nose and Throat Disorders.

\section{Description of the condition}

People with cancer may experience nausea and vomiting at different stages of their illness: before, during, and after chemotherapy or radiotherapy; and during a palliative phase of illness.

Glare 2011 defined nausea as, "...an entirely subjective experience... that immediately precede vomiting" [sic). Vomiting is a highly specific physical event and is defined as "the rapid, forceful evacuation of gastric contents in retrograde fashion from the stomach up to and out of the mouth".

Kris 2011 observed that, "one of the largest misconceptions in the field of oncology today is that the problem of ChemotherapyInduced Nausea and Vomiting (CINV), has been solved" whilst noting that "CINV is the most significant side effect of chemotherapy from the patient's perspective". The prevalence of CINV has been reported to be: $36 \%$ for acute CINV (within 24 hours of administration of chemotherapy); 59\% for delayed CINV (two to five days after administration of chemotherapy); and $47 \%$ for both (Cohen 2007). The cost per return hospital visit for CINV to the USA healthcare system, likely to occur if CINV remains uncontrolled, has been stated to be USD 5299 (Burke 2011). Nausea and vomiting, particularly CINV, are also known to have a negative impact on quality of life (Sommariva 2016), even when only moderately emetogenic chemotherapy (MEC), is administered. While the treatment of CINV has greatly improved over time, some people experience nausea and vomiting despite optimal treatment.

The prevalence of nausea in the palliative population has been estimated to range from $6 \%$ to $68 \%$ (Solano 2006). A systematic review of symptom prevalence in people with terminal cancer reported a pooled prevalence of $31 \%$ ( $95 \% \mathrm{Cl} 27$ to 35), for nausea and $20 \%$ ( $95 \% \mathrm{Cl} 17$ to 22 ), for vomiting (Teunissen 2007). Persistent nausea and vomiting in people with palliative conditions can lead to unplanned hospital visits. In one study, gastrointestinal symptoms, including nausea and vomiting, were the most common reason for a participant receiving palliative care to attend the emergency department ( $48 \%$ of participants), despite one third preferring not to be cared for in hospital (Hjermstad 2013).

This review will address the use of olanzapine in adults with cancerrelated nausea and vomiting at each stage of the illness: before, during, and after chemotherapy or radiotherapy; and during the palliative phase of illness.

\section{Description of the intervention}

The use of olanzapine for the prevention and treatment of nausea and vomiting has been reported in the literature in the form of case reports and case series since 2000 (Jackson 2003; Pirl 2000). These articles described its use for CINV and for palliation. Prommer 2012 went on to identify olanzapine as a drug of multi-palliation, "... being useful for the management of several symptoms commonly encountered in palliative care, such as delirium, nausea, vomiting, and pain".
There has been a growing interest in establishing the safety and efficacy of olanzapine as an antiemetic, particularly in the prevention and treatment of CINV, through randomised controlled trials (RCTs). The use of olanzapine as an antiemetic represents a new use of an old second generation atypical antipsychotic drug. Phase I, II, and III trials have been undertaken to assess its efficacy and tolerability in the prevention and treatment of CINV.

Olanzapine is available in many countries, in both branded and generic forms. It is most often administered orally, either as a tablet $(2.5 \mathrm{mg}$ to $20 \mathrm{mg}$ ), velotab, as an oral solution, or orallydisintegrating 'wafer', but it may also be given by intramuscular injection. Olanzapine has also been used in the treatment of cancerrelated cachexia, as it is known to promote weight gain, and in the treatment of delirium (Kishi 2015; Navari 2010).

In this review we have only identified RCTs describing oral administration. Furthermore, the literature has raised serious safety concerns relating to respiratory distress following intravenous administration (Martel 2016). These include hypoxia and the need for airway and ventilatory support. Therefore, the findings of this review cannot be extrapolated to justify use of the injectable form for intravenous, intramuscular or subcutaneous administration for the treatment or prevention of nausea and vomiting at the present time.

\section{How the intervention might work}

Olanzapine is a second generation, atypical thienobenzodiazepine antipsychotic with a broad spectrum of neurotransmitter blockade including serotonin 5-HT2a, 5-HT2c, 5-HT3, 5-HT6 receptors, dopamine D1, D2, D3, D4 brain receptors, $\propto 1$ adrenergic receptors, acetylcholine (ACh), muscarinic receptors, and $\mathrm{H} 1$ histamine receptors. This broad spectrum of action, particularly on the dopamine and serotonin receptors, has led to increasing interest in its use as an antiemetic, as it is likely to act at the vomiting centre and chemoreceptor trigger zone (Navari 2013a).

Olanzapine's broad spectrum of action in the prevention and treatment of nausea and vomiting may be similar to that of levomepromazine.

Olanzapine is known to cause adverse effects. The most relevant of these include weight gain, sedation, loss of control of diabetes mellitus, and blood dyscrasias. Extra-pyramidal side effects and dyskinesias, as well as prolongation of the corrected QT interval, have also been noted within this class of medications (BNF). The QT interval, measured on an Electrodariogram (ECG) as the time between the $\mathrm{Q}$ and $\mathrm{T}$ waves, is a measure of the time taken for the heart to repolarise and is corrected to take account of the heart rate. Prolongation of the QT interval is a risk factor for sudden death due to the increased potential of malignant ventricular tachyarrhythmias.

\section{Why it is important to do this review}

Given the emerging use of olanzapine as an antiemetic in both CINV and palliative care, it is important to establish the evidence base for its use in the prevention and treatment of nausea and vomiting. Nausea and vomiting during a patient's cancer journey is a common symptom. The antiemetics currently available are not effective for all people, particularly for the prevention or treatment of nausea. Providing another treatment option may provide relief to more 
people whose nausea is currently not relieved, and in situations where other antiemetics are unavailable.

Olanzapine is currently listed as an antipsychotic, however, it is not licensed for the prevention or treatment of nausea and vomiting in the UK by the Medicines and Healthcare products Regulatory Agency (MHRA), or in the USA by the Food and Drug Administration (FDA). Its use as an antiemetic is therefore currently an off-licence use in the UK and USA.

\section{OB JECTIVES}

To assess the efficacy and safety of olanzapine when used as an antiemetic in the prevention and treatment of nausea and vomiting related to cancer in adults.

\section{METHODS}

\section{Criteria for considering studies for this review}

\section{Types of studies}

We included randomised controlled trials (RCTs; described as 'randomised' anywhere in the manuscript), of olanzapine for the prevention or treatment of nausea or vomiting, or both, in any setting. We considered open-label studies. We only used the first phase of cross-over studies. We excluded quasi-randomised trials. Included studies required a minimum of 10 participants per treatment arm.

We excluded non-randomised studies, case series and case reports, and clinical observations. Studies were required to be fully published or available as extended abstracts (for example from clinical trials websites); we did not include short (usually conference), abstracts unless we had received satisfactory additional information the author through personal correspondence.

\section{Types of participants}

We included studies of inpatients or outpatients with cancer (of any type or stage), who had nausea and vomiting treated with olanzapine, or where olanzapine was used to prevent nausea and vomiting. Adult participants were male or female, and aged 18 years and older.

We excluded studies in which olanzapine was used for the treatment or prevention of nausea and vomiting in non-cancer patients.

\section{Types of interventions}

We included studies that gave olanzapine for the prevention or treatment of nausea and vomiting and compared it with placebo or any active comparator, by any route, in any dose, frequency, or duration of treatment. For the purpose of this review, we defined prevention as the use of an antiemetic prior to the first cycle of chemotherapy.

The main comparators were placebo, usual care, prokinetics (metoclopramide or domperidone), serotonin (5-HT3), receptor antagonists, and neurokinin 1 (NK1), receptor antagonists.

The main comparison pairs were:

- olanzapine versus placebo;
- olanzapine versus usual care.

Other possible comparison pairs, if data were available, were:

- olanzapine versus prokinetics;

- olanzapine versus 5-HT3 antagonists;

- olanzapine versus NK1 antagonists;

- olanzapine plus dexamethasone versus any possible comparators noted above plus dexamethasone.

\section{Types of outcome measures}

\section{Primary outcomes}

- Absence of nausea or vomiting as measured by the proportion of participants with no nausea or vomiting over the time period studied

- Serious adverse events, specifically, extra pyramidal adverse events, prolonged QTc interval, neutropenia and agranulocytosis, as measured by the proportion of participants experiencing at least one of these events

\section{Secondary outcomes}

- Patient perception of treatment including: patient satisfaction as measured by any scale including Likert; patient preference (as a dichotomous yes/no outcome); validated quality-oflife measures; number of participants with Patient Global Impression of Change (PGIC), of much improved or very much improved (or equivalent wording)

- Other adverse events, as measured by the proportion of participants experiencing at least one of these

- Somnolence and fatigue, as measured by the proportion of participants experiencing at least one of these (Differences between protocol and review)

- Attrition: withdrawals due to all causes (including lack of efficacy, adverse events, and death)

- Number of participants with at least a $50 \%$ reduction in the severity of nausea or vomiting from baseline, as measured by a validated scale

- Number of participants with breakthrough nausea and vomiting, and number of participants requiring rescue antiemetics

- Number of participants with no overall nausea and no overall vomiting, no anticipatory (prior to chemotherapy), nausea or vomiting, no acute (within 24 hours of administration of chemotherapy), nausea or vomiting, and no delayed (two to five days after administration of chemotherapy, nausea or vomiting

We did not exclude studies solely on the basis that the data were not available relating to any of these outcomes.

\section{Search methods for identification of studies}

The Cochrane Pain, Palliative and Supportive Care Information Specialist conducted the search. There were no language, publication year, or publication status restrictions. We arranged translations of papers where necessary.

\section{Electronic searches}

We searched the following databases without language restrictions for published data: 
- the Cochrane Central Register of Controlled Trials (CENTRAL; 2017, issue 9), via the CRSO;

- MEDLINE and MEDLINE in Process (Ovid), 1946 to 19 September 2017;

- Embase (Ovid), 1974 to 2017 week 38.

See Appendix 1 , Appendix 2 and Appendix 3 for the search strategies.

\section{Searching other resources}

We searched the following unpublished databases without language restrictions:

- ClinicalTrials.gov;

- apps.who.int/trialsearch/.

In addition, we checked reference lists of reviews identified in the search and retrieved full-text articles for additional studies on key articles. We contacted experts in the field for unpublished and ongoing trials. We contacted study authors where necessary for additional information.

\section{Data collection and analysis}

\section{Selection of studies}

Using Covidence, at least two review authors (AS, $\mathrm{KH}$ and $\mathrm{KN}$ ), screened the titles and abstracts of all identified studies, and excluded those that clearly did not meet the inclusion criteria. For the remaining studies, we read the full manuscript to assess if it should be included. A minimum of two review authors (AS, KH and $\mathrm{KN}$ ), independently screened all titles, abstracts and full-text manuscripts. We resolved discrepancies between review authors by discussion and consensus; where necessary, we consulted a third review author (BW). We did not anonymise studies before selection.

We have presented a PRISMA flow chart in Figure 1 (Moher 2009). This shows the status of identified studies, as recommended in Part 2, Section 11.2.1 of the Cochrane Handbook of Systematic Reviews of Interventions (Schünemann 2011). We included studies in the review regardless of whether the measured outcome data were reported in a 'usable' way. 


\section{Figure 1. Study flow diagram}

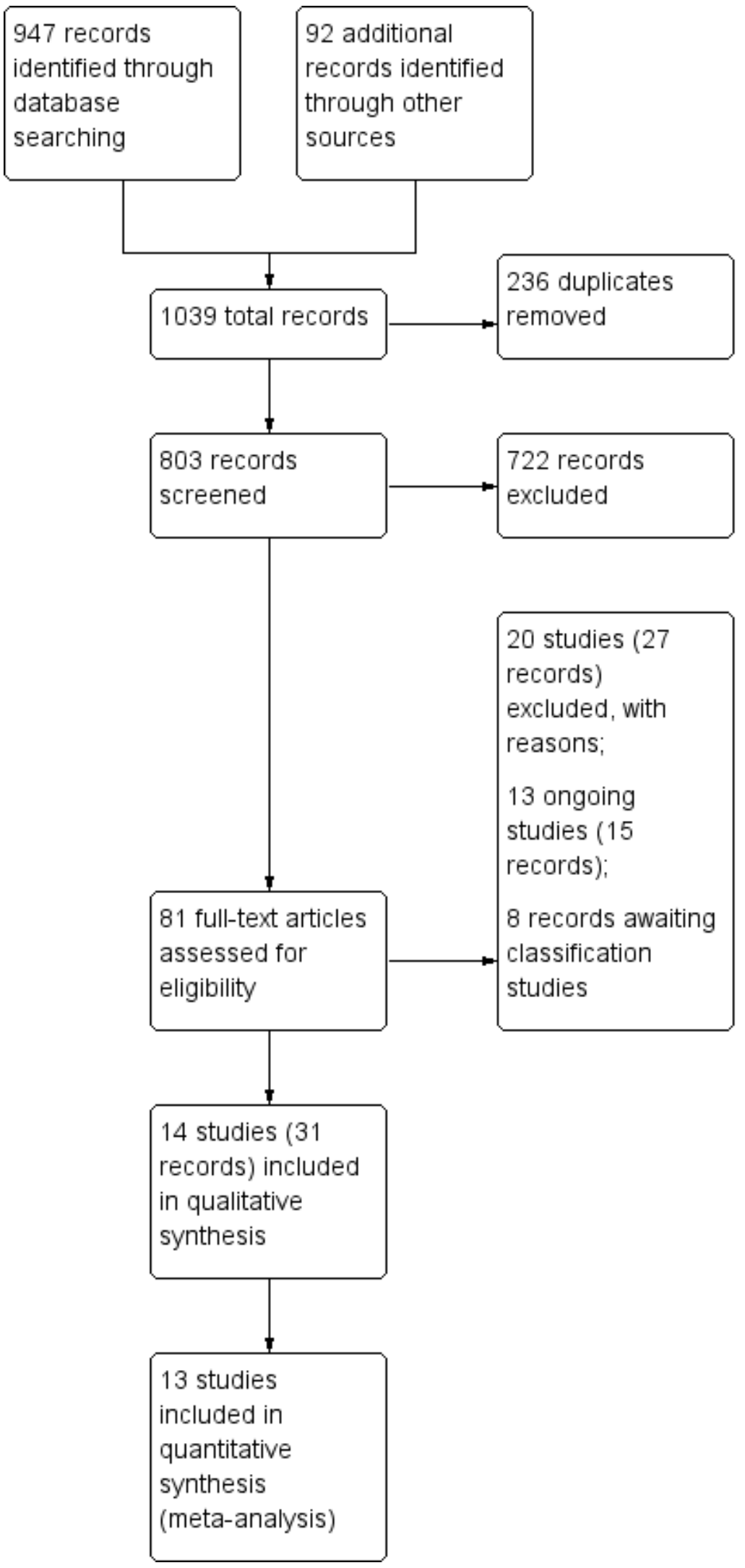




\section{Data extraction and management}

Four review authors (AS, LW, KH and EP), extracted data from the included studies using a standardised data collection form. All included articles were data extracted independently by a minimum of two review authors. Where there were differences in the data extracted by different review authors, we resolved this by reference to the original publications and through discussion and consensus, involving a third review author as necessary before entering them into Review Manager 5 (RevMan 5), (Review Manager 2014). Where a study had more than one publication, we retrieved all publications to ensure complete extraction of data. Where data were missing or unclear, we contacted the original study authors for clarification. We assessed the data using intention-to-treat as the basis for our data analysis. Where data were reported in divided groups following post hoc analysis without stipulating that stratified randomisation had been undertaken, we recombined the groups when extracting the data to mitigate the potential for breaking randomisation (Zhang 2017).

We included key information from each of the included studies in sufficient detail to populate a table of Characteristics of included studies, including: study design (placebo or active control, and cross-over), and methods, sample size, baseline demographic details, type of chemotherapy or radiotherapy received, if any, type of cancer, drug and dosing regimen (including when olanzapine is used in combination with another).

In addition to the pre-specified information about study characteristics and aspects of methodology relevant to risk of bias (see below), we extracted the following summary statistics for each trial and each outcome.

- For continuous data: the mean values, standard deviations, and number of participants for each treatment group. Where endpoint data were not available, we extracted the values for change from baseline.

- For dichotomous data: the numbers of participants experiencing an event and the number of patients randomised.

- For ordinal scale data: if the data appeared to be approximately normally distributed, or if the analysis that the investigators performed suggested parametric tests were appropriate, then we would have treated the outcome measures as continuous data. Alternatively, if data were available, we planned to convert them into dichotomous data.

\section{Assessment of risk of bias in included studies}

Four review authors (AS, KH, LW and EP), independently assessed the risk of bias for each included study using the criteria outlined in the Cochrane Handbook for Systematic Reviews of Interventions, and we used the Cochrane 'Risk of bias' table in RevMan 5, with any disagreements resolved by discussion (Higgins 2017; Review Manager 2014).

We assessed risk of bias as low, high, or unclear for each of the following six domains for each study.

- Random sequence generation (checking for possible selection bias). We assessed the method used to generate the allocation sequence as: low risk of bias (any truly random process, e.g. random number table; computer random number generator); unclear risk of bias (method used to generate sequence not clearly stated). We excluded studies using a non-random process (e.g. odd or even date of birth; hospital or clinic record number).

- Allocation concealment (checking for possible selection bias). The method used to conceal allocation to interventions prior to assignment determines whether intervention allocation could have been foreseen in advance of, or during recruitment, or changed after assignment. We assessed the methods as: low risk of bias (e.g. telephone or central randomisation; consecutively numbered, sealed, opaque envelopes); unclear risk of bias (method not clearly stated). We included studies that did not conceal allocation (e.g. open list), but considered them to have high risk of bias.

- Blinding of participants and personnel (checking for possible performance bias). We assessed the methods used to blind study participants and personnel from knowledge of which intervention a participant received. We assessed methods as: low risk of bias (study states that it was blinded and describes the method used to achieve blinding, such as identical tablets matched in appearance or smell, or a double-dummy technique); unclear risk of bias (study states that it was blinded but does not provide an adequate description of how it was achieved). We considered studies that were not double-blind to have high risk of bias.

- Blinding of outcome assessment (checking for possible detection bias). We assessed the methods used to blind study participants (for participant-reported outcomes), and outcome assessors from knowledge of which intervention a participant received. We assessed the methods as: low risk of bias (study had a clear statement that outcome assessors were unaware of treatment allocation, and ideally described how they achieved this); unclear risk of bias (study stated that outcome assessors were blinded to treatment allocation but lacked a clear statement on how they achieved this). Studies where outcome assessment was not blinded we considered as having a high risk of bias.

- Selective reporting (checking for reporting bias). We assessed whether primary and secondary outcome measures were prespecified and whether these were consistent with those reported. We assessed the methods as: low risk of bias (where prespecified outcome measures were available and reflected the published results); unclear risk of bias (where no protocol was available); and high risk (where published results did not reflect prespecified outcome measures).

- Incomplete outcome data (checking for possible attrition bias due to the amount, nature and handling of incomplete outcome data). We assessed the methods used to deal with incomplete data as: low risk $<10 \%$ of participants did not complete the study and/or used 'baseline observation carried forward' analysis); unclear risk of bias (used 'last observation carried forward' analysis); high risk of bias (used 'completer' analysis).

- Size of study (checking for possible biases confounded by small size). We assessed studies as being at low risk of bias ( $\geq 200$ participants per treatment arm); unclear risk of bias (50 to 199 participants per treatment arm); and high risk of bias $(<50$ participants per treatment arm).

We defined studies at high risk of bias overall as those that had a high risk of bias within the random sequence generation or allocation concealment domains, or both, and a high risk of attrition bias (overall loss to follow-up greater than $20 \%$, differential follow-up observed, or both). If further information was 
not available, this was reflected in a designation of unclear risk of bias overall (Higgins 2017).

\section{Measures of treatment effect}

We summarised the effects of dichotomous outcomes by using risk ratios (RR), with $95 \%$ confidence intervals $(\mathrm{Cl})$, but noted that this appeared to underestimate the heterogeneity in the data set largely due to the differences in the event rates between studies. We therefore decided to also present the data using the odds ratio (OR), in the results and 'Summary of findings' table as this provided a truer reflection of heterogeneity in the $\mathrm{I}^{2}$ value, although we recognise that this is not as easily interpreted as the RR in clinical practice.

For continuous outcomes, we expressed treatment effects as a mean difference (MD), with standard deviation (SD), or as standardised mean difference (SMD), if studies used different scales to measure the same outcome. We provided a clinical interpretation of the SMD values.

For the key outcomes presented in the 'Summary of findings' table, we also expressed the results as absolute numbers, based on the pooled results and compared to the assumed risk. We calculated the number needed to treat for an additional beneficial outcome (NNTB), using the pooled results. The assumed baseline risk was either (a), the median of the risks of the control groups in the included studies (this being used to represent a medium risk population), or alternatively, (b), the average risk of the control groups in the included studies, this being the study population (Deeks 2017).

If a large number of studies had been available, and if it were appropriate, we would have also presented additional data based on the assumed baseline risk in (c), a low-risk population, and (d), a high-risk population.

For unwanted effects, we calculated the number needed to treat for an additional harmful outcome (NNTH), in the same manner.

We used the following terms to describe adverse outcomes in terms of harm or prevention of harm.

- When significantly more participants experienced adverse outcomes with olanzapine compared with control (placebo or active), we used the term, number needed to harm or cause one event.

- If significantly fewer participants had experienced adverse outcomes with olanzapine than with control (placebo or active), we would have used the term number needed to treat to prevent one event (NNTp).

We did not plan to use continuous data for the primary outcome because it was inappropriate where there is an underlying skewed distribution, as is likely to be the case with nausea and vomiting response reporting.

\section{Unit of analysis issues}

The unit of randomisation was the individual participant. We excluded trials comparing differing doses of olanzapine in this review. However, in future updates we intend to include these. In these studies comparing differing doses of olanzapine, if data were not combined for analysis, we planned to split the control treatment arm between active treatment arms. Where only combined data for different periods (such as anticipatory, acute or delayed), were reported, we would treat the study as if it were a parallel study, drawing attention to the potential bias that this confers, and interpreting the results accordingly.

\section{Dealing with missing data}

We used an intention-to-treat (ITT), analysis, that is, participants who were randomised, took the study medication, and gave a minimum of one post-baseline assessment. Where there were missing participants or information, we assigned them to a zero improvement category, where possible, for the efficacy outcomes (e.g. assigned them to having nausea or vomiting). We also looked for information about how data from withdrawals and dropouts were handled. In original studies, participants might have been analysed using last observation carried forward (LOCF, that is, their level of nausea or vomiting when stopping the medication), or returned to their baseline observation (BOCF, baseline observation carried forward).

Where there were substantial numbers (more than 10\%), of participants missing from analyses, we commented on this.

Where data relating to an outcome of interest were not reported, but the methods of the study suggested that the outcome had been measured, we attempted to contact study authors by email to obtain this information. We also did this if some of the data required for meta-analysis were unreported, unless the missing data were SDs.

If SD data were not available, we approximated these using the standard estimation methods from $\mathrm{P}$ values, standard errors, or 95\% Cls, if these were reported, as detailed in the Cochrane Handbook for Systematic Reviews of Interventions (Deeks 2017). If it was impossible to estimate these, we contacted the study authors.

We did not make any imputations apart from those for missing standard deviations. We extracted and analysed all data using the ITT method.

\section{Assessment of heterogeneity}

We assessed both clinical and statistical heterogeneity. Clinical heterogeneity might be present even in the absence of statistical heterogeneity. We anticipated that there might be an effect of differences between participants, environment (inpatient versus outpatient), and outcome measures. We examined the included trials for evidence of differences in the types of participants recruited, setting, dose, and route of interventions, controls, or outcomes measured.

We assessed statistical heterogeneity by visually inspecting the forest plots and by considering the $\mathrm{I}^{2}$ statistic (Higgins 2003). This calculates the percentage of variability that is not due to chance. $I^{2}$ statistic values over $50 \%$ suggest substantial heterogeneity (Deeks 2017).

If heterogeneity $\left(I^{2}\right)$, was high, we investigated this. We explored this with subgroup and sensitivity analyses where there were sufficient data. 


\section{Assessment of reporting biases}

We assessed two aspects of reporting bias: between-study publication bias and within-study outcome reporting bias.

\section{Publication bias (between-study reporting bias)}

If we had suspected publication bias, we would have conducted a more formal investigation using the methods proposed by Egger 1997.

\section{Outcome reporting bias (within-study reporting bias)}

We assessed within-study reporting bias by comparing the outcomes reported in the published report with those listed in the study protocol whenever possible, or, if the protocol was not available, with those listed in the methods section. If results were mentioned in the protocol or methods section, but were not reported in a way that allowed analysis, we sought further information from the study authors in order to try and reduce bias in the meta-analysis.

\section{Data synthesis}

We used RevMan 5 to carry out meta-analyses (Review Manager 2014). Where possible, we analysed data to give a summary measure of effect. If no or minimal heterogeneity was seen, we used a fixed-effect model for meta-analysis to measure the effect. Where considerable heterogeneity was observed, we used a randomeffects model.

For dichotomous data, we analysed treatment differences as a risk ratio (RR), calculated using the Mantel-Haenszel methods. If we had found any time-to event data we would have analysed them using the generic inverse variance method.

For continuous outcomes, if all the data were from the same scale, we pooled mean values obtained at follow-up (endpoint data), with change outcomes, and reported this as a MD. If the SMD had to be used as an effect measure, we did not pool endpoint and change data.

When statistical heterogeneity was low, the differences in treatment effects seen when using methods based on a randomeffects versus a fixed-effect model were trivial. When statistical heterogeneity was high, we used the random-effects method, as this provides a more conservative estimate of the difference.

\section{GRADE and 'Summary of findings' table}

We used the GRADE approach to rate the overall quality of evidence (Guyatt 2011). We used the GRADEpro GDT tool listed in the Types of interventions section (GRADEpro GDT 2015, accessed 30th October 2017). The quality of evidence reflects the extent to which we are confident that an estimate of effect is correct, and we applied this in the interpretation of results. There are four possible ratings: high, moderate, low, and very low. A rating of high-quality evidence implies that we are very confident that the true effect lies close to that of the estimate of the effect. A rating of very low-quality implies that we have very little confidence in the effect estimate and the true effect is likely to be substantially different from the estimate of effect.

The GRADE approach rates evidence from RCTs that do not have any serious limitations as high quality. However, several factors can lead to the downgrading of the evidence to moderate, low, or very low. The degree of downgrading was determined by the seriousness of these factors:

- study limitations (risk of bias);

- inconsistency;

- indirectness of evidence;

- imprecision;

- publication bias.

We decreased the grade rating by one $(-1)$ or two (- 2) (up to a maximum of - 3 to 'very low') if we identified:

- serious (-1) or very serious (- 2) limitation to study quality;

- important inconsistency (-1);

- some (-1) or major (-2) uncertainty about directness;

- imprecise or sparse data (-1);

- high probability of reporting bias $(-1)$.

We have included two 'Summary of findings' tables to present the main findings in a transparent and simple tabular format according to the recommendations described in the Cochrane Handbook for Systematic Reviews of Interventions (Schünemann 2017). In particular, we have included key information concerning the quality of evidence, the magnitude of effect of the interventions examined, and the number of participants in which the effect was observed from the available data on the following outcomes:

- absence of nausea or vomiting, as measured by the proportion of participants with no nausea or vomiting over the time period studied;

- serious adverse events (specifically extra-pyramidal adverse events, prolonged QTc, neutropenia, and agranulocytosis), as measured by the proportion of participants experiencing at least one of these events;

- participant satisfaction or preference;

- quality-of-life measures;

- number of participants with Patient Global Impression of Change (PGIC), of much improved or very much improved (or equivalent wording);

- other adverse events (e.g. somnolence), as measured by the proportion of participants experiencing at least one of these.

\section{Subgroup analysis and investigation of heterogeneity}

We conducted some subgroup analyses even if we did not observe statistical heterogeneity. We planned these analyses as the factors indicated are suspected to be potential effect modifiers. This was particularly the case when comparing participants with a cancer diagnosis who were receiving active treatment to those in a palliative phase of their illness.

We have presented the main analyses of this review without dividing by subgroup. Where subgrouping was possible, we have presented all of the results of the subgroup analyses in the Effects of interventions section of the review, and as forest plots. We have discussed the clinical importance of the analyses in the discussion section.

Where there were sufficient data, we used subgroup analysis to establish: 
- the effect of dose (2.5 mg versus $5 \mathrm{mg}$ versus $10 \mathrm{mg}$ twice daily), on efficacy and adverse events. This was in order to establish the dose response effect of olanzapine in the context of nausea and vomiting, in order to identify the lowest effective dose;

- the efficacy of olanzapine in different clinical settings, such as patients undergoing chemotherapy, patients undergoing radiotherapy, or no active oncology treatment. This was because the mechanisms of nausea and vomiting are thought to differ in each of these settings, and therefore, may affect the efficacy of olanzapine as an antiemetic;

- the efficacy of olanzapine in moderately versus highly emetogenic chemotherapy. The proportion of participants expected to suffer from nausea and vomiting are different in MEC versus HEC, and therefore, the degree of emetogenicity of the chemotherapy may affect the efficacy of olanzapine as an antiemetic.

\section{Sensitivity analysis}

Where necessary we carried out sensitivity analyses to determine whether the findings were robust, based on the decisions made in undertaking the review. We planned analyses for the following factors:

- model chosen: fixed-effect versus random-effects;

- risk of bias of included studies (excluding studies with high risk of bias);

- methods of outcome measurement (evaluating the impact of including data where the validity of the measurement was unclear)

\section{RES U LTS}

\section{Description of studies}

\section{Results of the search}

The planned searches of published databases identified 947 potentially relevant records and 92 additional records were identified through other sources. Of these, 41 were unpublished records found in clinical trials registries. We screened titles and abstracts, screening the full texts where necessary. We excluded 722 records during the screening process.

We assessed full texts of 81 potentially relevant records. We included 13 published studies (30 records), and one unpublished study. We excluded 20 studies (27 records), with reasons, including four RCTs in paediatric populations. We identified 13 ongoing studies (15 records), and eight studies that are awaiting classification.

A flow chart of study retrieval and selection is provided in Figure 1.

\section{Included studies}

We included 14 RCTs, with a total of 1917 participants (Clemons 2016; Liu/Tan 2015; Lu 2013; Mizukami 2014; Mukhopadhyay 2016; Nakagaki 2017; Navari 2010b; Navari 2013b; Navari 2016a; Nikbakhsh 2016; Shumway 2015; Wang 2015; Zhang 2017; Zhao 2014). Thirteen of these reported at least one of our prespecified outcome measures and contributed data to the meta-analysis. One RCT was included in our qualitative synthesis but did not contribute data to the quantitative analysis (Clemons 2016). See Characteristics of included studies.

\section{Trial design}

All of the studies were RCTs ranging in size from 20 to 401 participants, were blinded or unblinded, parallel-arm design. Most trials had two comparator arms, whilst one had three arms (Nakagaki 2017). We were able to obtain data for all included RCTs except for one study, which did not contribute data to our meta-analysis as it compared a risk stratification model of care to physician choice, rather than directly assessing the olanzapine versus a comparator (Clemons 2016).

\section{Comparison pairs}

The comparisons in the included studies varied. Nine studies compared olanzapine to placebo or no treatment. Four studies compared olanzapine to other antiemetics: NK1 antagonists (one study Shumway 2015); 5-HT3 antagonists (one study Nakagaki 2017); prokinetics (one study Navari 2013b); and one study compared olanzapine to steroids (Liu/Tan 2015). All participants in all of the trials had treatment in conjunction with various other medications, chiefly antiemetics.

\section{Population}

The participants were both male and female with ages ranging from 18 to 81 years. Participants had a range of histologically diagnosed cancer types. Solid and haematological malignancies were represented within the included trials. These included bladder, breast, cervical, colorectal, gingival, glioblastoma, head and neck cancer, laryngeal, lung, leukaemia, lymphoma (including Hodgkins lymphoma), malignant melanoma, oesophageal, oropharyngeal, osteosarcoma, ovarian, pancreatic, pharynx, sarcoma, stomach, teratoma and thymus.

We did not identify any RCTs that included participants who were receiving radiotherapy without concurrent chemotherapy. One RCT included participants who were receiving both chemotherapy and radiotherapy treatment for their cancer (Nakagaki 2017). Participants in Nakagaki 2017 were undergoing hematopoietic stem cell transplantation. They received, "conditioning regimens [that) included high-dose melphalan $(200 \mathrm{mg} / \mathrm{m2})$ and BEAM for autologous HSCT and $\mathrm{Cy} / \mathrm{TBI}$ and fludarabine/melphalan (melphalan $120 \mathrm{mg} / \mathrm{m} 2$ ) for allogeneic protocols" and some also received total body irradiation.

One RCT included participants who were not receiving either chemotherapy or radiotherapy for their cancer (Navari 2010b), and instead received olanzapine with megestrol or megestrol alone.

\section{Setting}

The countries of origin represent both high-, middle- and lowincome countries, including: Australia (Nakagaki 2017); Canada (Clemons 2016); China (Liu/Tan 2015; Lu 2013; Wang 2015; Zhang 2017; Zhao 2014); Iran (Nikbakhsh 2016); India (Mukhopadhyay 2016); Japan (Mizukami 2014); and the USA (Navari 2010b; Navari 2013b; Navari 2016a; Shumway 2015). The included articles were published in English and Chinese. Non-English language papers were translated and data extracted when necessary.

The trials were conducted in hospital settings including university hospitals, a military medical centre, oncology centres and a bone marrow transplant centre. Trials recruited both outpatients and inpatients. The number of centres where the trials were conducted ranged from single centres to multicentre recruitment models, with 
the largest trial recruiting participants from 46 different centres around the USA (Navari 2016a).

\section{Primary outcomes}

Only two (Lu 2013; Mukhopadhyay 2016), out of 13 studies contributing to the meta-analyses reported the primary outcome of the number of participants who were free from both nausea and vomiting over the whole time period. Instead, most studies reported the number of participants with no vomiting and no use of breakthrough antiemetic medication, which they defined as "complete response". Additionally, two studies did not report serious adverse events (Liu/Tan 2015; Navari 2013b).

Most studies reported adverse effects of treatment using defined standards. When reviewing the studies we noted that somnolence or fatigue were the most frequently reported adverse events. We felt that these have a large impact on patients and so we decided, as a change from protocol, to investigate this adverse event separately from other adverse events (see Differences between protocol and review).

\section{Funding}

None of the included RCTs declared having received funding from pharmaceutical companies. Of the studies that reported having received funding support, two were supported by universities (Zhang 2017; Nikbakhsh 2016), one by a hospital foundation Nakagaki 2017, one by an endowed fund (Navari 2013b), one by a cancer foundation (Clemons 2016), and one study received funding from both an endowed fund and a cancer foundation (Navari 2010b). Seven RCTs did not provide information as to how the studies were funded (Liu/Tan 2015; Lu 2013; Mizukami 2014; Navari 2016a; Shumway 2015; Wang 2015; Zhao 2014), and one study reported that "the authors have no financial disclosure, and the study was not a sponsored study" (Mukhopadhyay 2016).

\section{Interventions}

All included studies administered olanzapine via the oral route only.

The most frequently reported dose was $10 \mathrm{mg}$ daily (as a single dose or divided doses of $5 \mathrm{mg}$ twice daily), reported in eight RCTs. four RCTs reported a $5 \mathrm{mg}$ daily dose (as a single dose or divided doses of $2.5 \mathrm{mg}$ twice daily). One trial reported a range of doses according to participant tolerance from $2.5 \mathrm{mg}$ daily up to $10 \mathrm{mg}$ daily (Nikbakhsh 2016), although the data are not presented in a way to make analysis of the efficacy of the $2.5 \mathrm{mg}$ dose possible. One trial used $2.5 \mathrm{mg}$ of olanzapine daily but could not be included in the meta-analysis (Clemons 2016).

Olanzapine can be administered orally, either as a tablet, an oral solution, or as an orally-disintegrating 'wafer' or 'velotab'. 10 RCTs did not state which oral preparation they used, one study used the tablet (Nikbakhsh 2016), two studies used the oral wafer (Mizukami 2014; Nakagaki 2017), and one study had "black gel capsule prepared by the central oncology pharmacy" so that they were indistinguishable from the black gel capsules used as a placebo (Shumway 2015).

\section{Standard adjuvant antiemetic regimens}

All included studies used olanzapine in conjunction with 'treatment as usual' (chiefly, antiemetics, although one study used megestrol), versus 'treatment as usual' plus placebo or no additional treatment.

Reported standard regimens used in the context of chemotherapyor chemoradiotherapy-induced nausea and vomiting included:

- a 5-HT3 antagonist (Wang 2015);

- a 5-HT3 antagonist and an NK1 antagonist (Nakagaki 2017);

- dexamethasone and a 5-HT3 antagonist (Mukhopadhyay 2016; Liu/Tan 2015; Shumway 2015);

- dexamethasone, an NK1 antagonist and a 5-HT3 antagonist (Mizukami 2014; Navari 2013b; Navari 2016a; Zhang 2017)

- dexamethasone, an antihistamine and a 5-HT3 antagonist (Lu 2013; Zhao 2014).

- standard regimen not stated (Nikbakhsh 2016)

These standard treatment regimens were used in both arms of the studies, therefore the presence or absence of olanzapine was the only difference between each intervention and control arm, enabling us to undertake meta-analysis. However, Clemons 2016 compared a "risk model-guided (RMG) antiemetic prophylaxis with physician's choice (PC)" resulting in the standard adjuvant antiemetic regimens varying between participants within each arm of the trial. This meant that the data from Clemons 2016 did not contribute to our quantitative analysis.

The study that contributed to the meta-analysis in adults with cancer not receiving chemotherapy or radiotherapy used olanzapine and megestrol versus megestrol alone to investigate the effect of olanzapine on cachexia and anorexia, in addition to its effect on nausea and vomiting (Navari 2010b).

\section{Possible confounding factors}

There were several issues with the interventions used in some of the included studies, either as standard treatment regimens or as comparators, that inhibited the certainty with which the observed effects could be attributed solely to olanzapine.

Mukhopadhyay 2016 used differing doses of dexamethasone for participants receiving MEC to those receiving HEC, however, this approach was consistent between the two comparator arms.

In Mizukami 2014 participants in both arms received a 5-HT3 antagonist. However a range of medications, doses and regimens were used from across the class of 5-HT3 antagonists including: day one to three granisetron, day one to two ondansetron, day one to three ramosetron, or day one palonosetron. It is not known if participants receiving each of these were matched pairs across the arms or if a consistent number of participants were randomised to each of the four different 5-HT3 antagonists.

Nikbakhsh 2016 failed to state the nature of the standard regimen.

Nakagaki 2017 used high doses of ondansetron (ondansetron 32 mg intravenous over 24 hours in one study arm and ondansetron eight $\mathrm{mg}$ intravenous three times day as standard therapy in the other arms), beyond the doses recommended by the manufacturer (Medicines.org a).

Lu 2013 and Zhao 2014 used diphenhydramine (Benadryl), in both comparator arms, which has a potent sedative effect when used alone. In addition to its anticholinergic and antimuscarinic effects, 
it is known to "potentiate the sedative effects of alcohol and other central nervous system (CNS), depressants (e.g. tranquillizers, hypnotics and anxiolytics)" (Medicines.org). It is plausible that diphenhydramine may exacerbate the somnolence effects of olanzapine when used in combination.

\section{Studies awaiting classification}

We identified eight RCTs that are awaiting classification. We are awaiting further information from the authors for four RCTs that were only published as abstracts (Chasick 2012; Jeon 2017; Mukesh 2017; Nguyen 2017).

Additionally, we are awaiting information from authors regarding three RCTs that meet our inclusion criteria:

- CTRCC-14004093 a completed RCT with no published or unpublished results available;

- Mao 2011 is a cross-over study where only the combined phase one and two data have been published; and

- Meng 2016 a study where the age range of participants has not been published.

One study was unobtainable despite multiple searches (Wang 2015). See Characteristics of studies awaiting classification.

\section{Ongoing studies}

We identified a further 13 ongoing studies (Abe 2017; ChiCTRTTRCC-14004093; Hashimoto 2017; JPRN-UMIN000010317; Mukhopadhyay 2017a; Nagashima et al 2015; NCT02290470; NCT02400866; NCT02635984; NCT02939287; NCT02970643; NCT03079219; NCT03137121). See Characteristics of ongoing studies.

\section{Excluded studies}

We excluded 20 studies. The reasons for exclusion were as follows.

- Three did not fit our definition of a RCT on full-text review, such as cohorts, case reports and case series (wrong study design), (EUCTR2015-002294-38-DK; Nakashima 2015; Slimano 2016).

- Two used the wrong intervention (Hashimoto 2016; Yanai 2015).

- Four studied olanzapine in the wrong population (paediatric), (Flank 2015a; Flank 2015b; Fountaine 2010; Long 2017).

- Four studies terminated early, for example due to low recruitment (NCT01148264; ISRCTN58624349; Guntsch 2012; NCT00124930).

- Five studies used the wrong comparison, comparing differing doses of olanzapine (Babu 2016; Navari 2009a; Navari 2010a; Navari 2011; Navari 2016b).

- Two used olanzapine for the wrong indication (Kwatra 2013; Mukhopadhyay 2012).

Five of these excluded RCTs used a higher dose of dexamethasone in the olanzapine arm than in the comparison arm and we excluded them as they did not assess the effect of olanzapine alone (Babu 2016, Navari 2011, Navari 2016b, Navari 2009a, Navari 2010a). Instead they used olanzapine in conjunction with dexamethasone $20 \mathrm{mg}$, alongside standard therapy, on day one in the intervention group and compared this to standard therapy using $12 \mathrm{mg}$ dexamethasone on day one in the control arm. See Characteristics of excluded studies.

\section{Risk of bias in included studies}

Figure 2 and Figure 3 present a summary of our assessment of the risk of bias in the included studies. 
Figure 2. Risk of bias summary: review authors' judgements about each risk of bias item for each included study

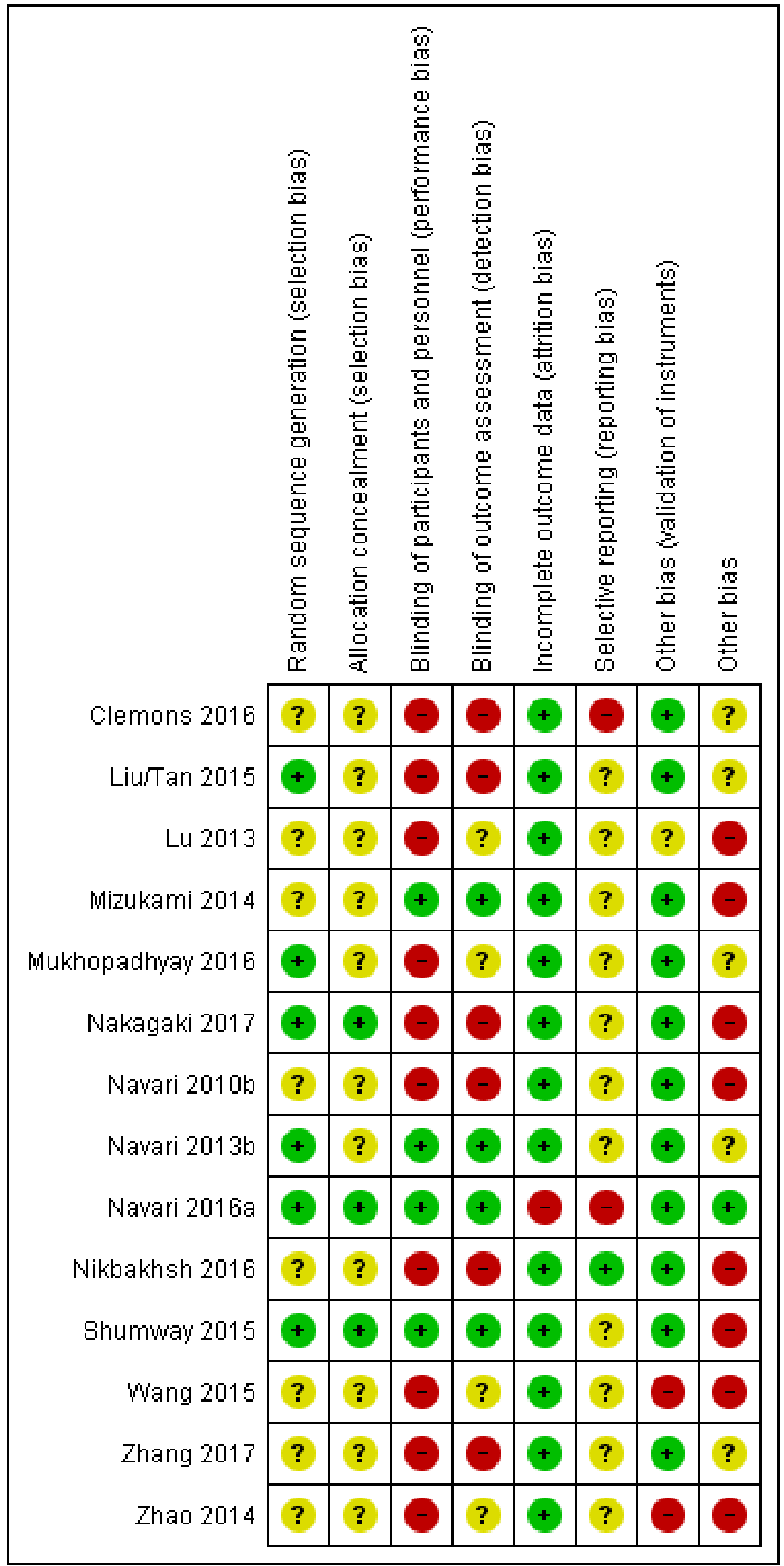


Figure 3. Risk of bias graph: review authors' judgements about each risk of bias item presented as percentages across all included studies

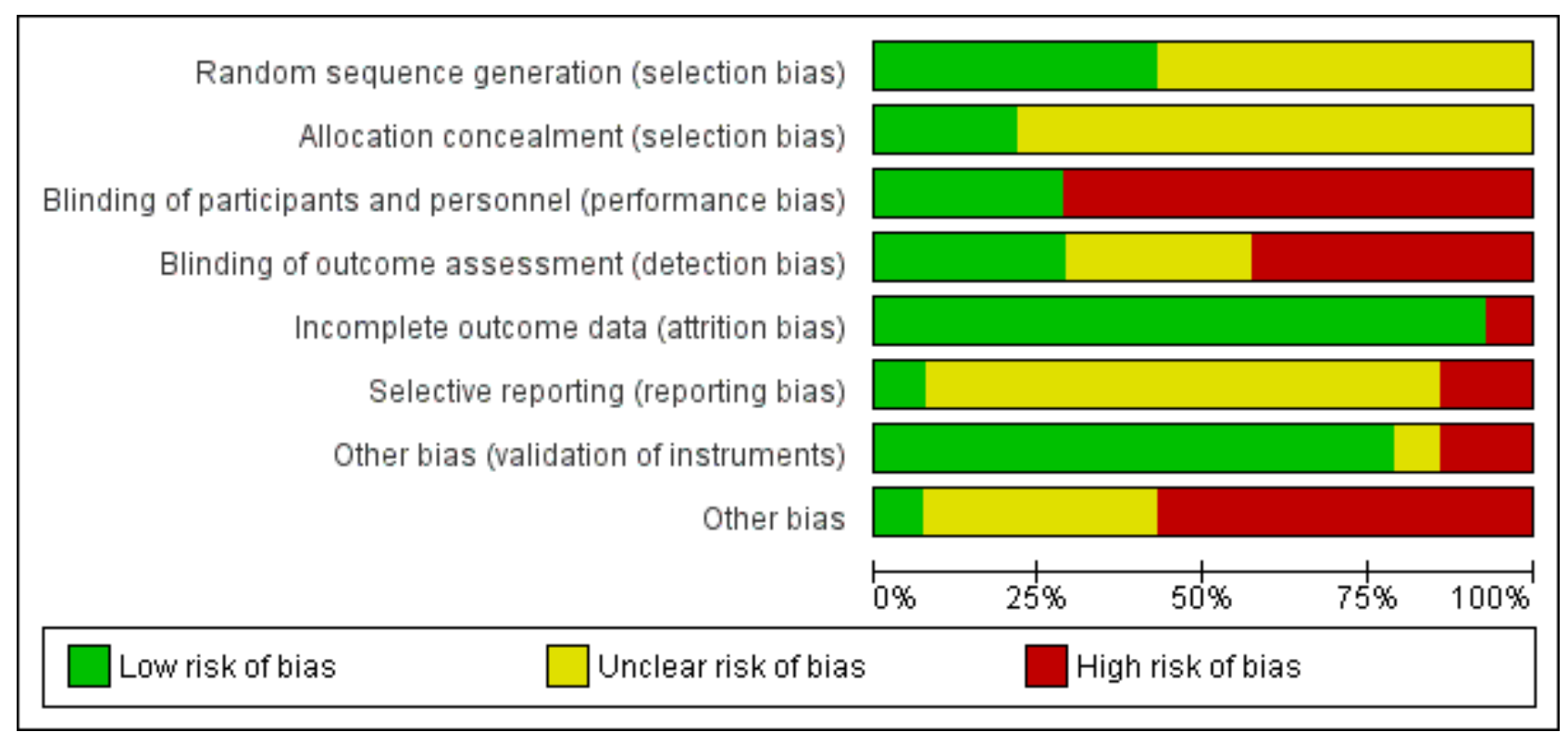

\section{Allocation}

\section{Random sequence bias}

In six RCTs, investigators used a table of random digits, computergenerated random numbers, or simple randomisation methods such as shuffling opaque envelopes to generate the allocation and we assessed them to be at low risk of bias (Liu/Tan 2015; Mukhopadhyay 2016; Nakagaki 2017; Navari 2013b; Navari 2016a; Shumway 2015). However, eight studies did not adequately describe their randomisation method and we therefore assessed them to be at unclear risk of bias (Clemons 2016; Lu 2013; Mizukami 2014; Navari 2010b; Nikbakhsh 2016; Wang 2015; Zhang 2017; Zhao 2014). We did not judge any studies at high risk of random sequence bias.

\section{Allocation concealment}

Three RCTs clearly described adequately concealing the allocation assigned to participants (Nakagaki 2017; Navari 2016a; Shumway 2015). However this was one of the least well reported areas of the 'Risk of bias' assessment, with 11 studies giving insufficient accounts or not making reference to it, hence we judged them to be at unclear risk of bias for this domain (Clemons 2016; Liu/Tan 2015; Lu 2013; Mizukami 2014; Mukhopadhyay 2016; Navari 2010b; Navari 2013b; Nikbakhsh 2016; Wang 2015; Zhang 2017; Zhao 2014).

\section{Blinding}

\section{Performance bias}

We assessed whether the personnel running the trials and the participants were blinded as to which intervention arm participants were allocated to. We assessed 10 studies as high risk (Clemons 2016; Liu/Tan 2015; Lu 2013; Mukhopadhyay 2016; Nakagaki 2017; Navari 2010b; Nikbakhsh 2016; Wang 2015; Zhang 2017; Zhao 2014), as they were unblinded studies. Only four studies described procedures used to blind participants and healthcare professionals and so we assessed them to be at low risk of performance bias (Mizukami 2014; Navari 2013b; Navari 2016a; Shumway 2015).

\section{Detection bias}

We assessed whether the person reporting the outcome was blinded as to which arm the participants were in.

Some trials asked the participants to self-report, typically through use of a daily diary. We assessed this method to be at low risk of bias if the participants were blinded and at high risk if they were not. Other trials used personnel to assess the outcome face to face or over the phone. Again, we assessed this method to be at low risk of bias if the personnel assessing the outcome were blinded and at high risk if they were not.

We assessed four studies to be at low risk of detection bias (Mizukami 2014; Navari 2013b; Navari 2016a; Shumway 2015); six at high risk (Clemons 2016; Liu/Tan 2015; Nakagaki 2017; Navari 2010b; Nikbakhsh 2016; Zhang 2017); and felt it was unclear in a further four studies (Lu 2013; Mukhopadhyay 2016; Wang 2015; Zhao 2014).

\section{Incomplete outcome data}

We assessed attrition of $10 \%$ of participants or more due to any reason as causing a study to be at high risk of attrition bias. We assessed all included studies to be at low risk of bias.

\section{Selective reporting}

We assessed studies that failed to report all outcomes set out in their study protocol (if available), or methods as being at high risk of selective reporting bias and those that reported all planned outcomes at low risk of selective reporting bias. Two trials were at high risk of reporting bias (Clemons 2016; Navari 2016a). We assessed Nikbakhsh 2016 to be at low risk of reporting bias. All other included studies were at unclear risk of selective reporting bias, as pre-planned outcomes were not available (Liu/Tan 2015; Lu 2013; Mizukami 2014; Mukhopadhyay 2016; Nakagaki 2017; Navari 2010b; Navari 2013b; Shumway 2015; Wang 2015; Zhao 2014). 
We assessed Zhang 2017 to be at unclear risk of bias because it is an unpublished study, where both the clinical study protocol and some of the results were available to us at clinicaltrials.gov (Protocol 02484911). It was clear that the outcomes had changed during the trial process. Some of the original outcomes that they had initially planned to report had not been reported (e.g. quality of life). In addition, the initial plan was not to analyse HEC and MEC populations separately, and this change appeared to be made after the trial had started. We await the full publication in order to assess this further.

\section{Other potential sources of bias}

\section{Use of a validated scale}

We assessed whether any scaled data was recorded using an appropriate validated scale. Validated scales reported in the trials that we assessed to be at low risk of bias included:

- visual analogue scale (VAS);

- Functional Living Index-Emesis (FLIE);

- European Organisation for Research and Treatment of Cancer Quality-of-Life questionnaire (EORTC QLQ-C30);

- MD Anderson Symptom Inventory (MDASI);

- Functional Assessment of Cancer Therapy - General (FACT-G), version 3;

- tool for assessing control of nausea and vomiting (The Multinational Association for Supportive Care in Cancer (MASCC)) scale;

- Hospital Anxiety and Depression Scale (HADS);

- World Health Organization Quality of Life Instruments (WHOQOL-BREF); and

- the Rhodes Index.

We were unable to find data relating to the validation of the WHO anticancer drug toxicity, WHO digestive tract reaction classification standard of anticancer drugs, grading criteria and Common Terminology Criteria for Adverse Events (CTCAE), version 3.0, which we therefore assessed to be at high risk of bias. We assessed Lu 2013 to be at unclear risk of bias; Wang 2015 and Zhao 2014 at high risk of bias; and 11 other studies at low risk of bias, based on the scales they reported using (Clemons 2016; Liu/Tan 2015; Mizukami 2014; Mukhopadhyay 2016; Nakagaki 2017; Navari 2010b; Navari 2013b; Navari 2016a; Nikbakhsh 2016; Shumway 2015; Zhang 2017).

\section{Size of study}

We also assessed the risk of bias according to the number of participants per arm. As is convention in Cochrane Pain, Palliative and Supportive Care (PaPaS) reviews, we assessed studies to be at low risk of bias if they had 200 or more participants in each treatment arm. We assessed studies to be at unclear risk of bias if they randomised 50 to 199 participants to each treatment arm, and we assessed trials with fewer than 50 participants in each treatment arm to be at high risk of bias.

Eight studies had a small sample size and therefore were at high risk of bias (Lu 2013; Mizukami 2014; Nakagaki 2017; Navari 2010b; Nikbakhsh 2016; Shumway 2015; Wang 2015; Zhao 2014). Five studies were of moderate size and were therefore at unclear risk of bias (Clemons 2016; Liu/Tan 2015; Mukhopadhyay 2016; Navari 2013b; Zhang 2017). Only one trial, which recruited 401 participants in total across the two arms, was at low risk of bias (Navari 2016a).

\section{Effects of interventions}

See: Summary of findings for the main comparison Olanzapine compared to placebo/no treatment for the prevention and treatment of cancer-related nausea and vomiting in adults; Summary of findings 2 Olanzapine compared to NK1 antagonist for the prevention and treatment of cancer-related nausea and vomiting in adults

Please see Summary of findings for the main comparison for the main comparison olanzapine versus placebo or no treatment.

Please see Summary of findings 2 for olanzapine versus NK1 antagonists.

\section{Olanzapine versus placebo or no treatment}

Nine studies ( $N=1000$ participants) compared olanzapine with placebo or no treatment (Lu 2013; Mizukami 2014; Mukhopadhyay 2016; Navari 2010b; Navari 2016a; Nikbakhsh 2016; Wang 2015; Zhang 2017; Zhao 2014).

Seven studies used olanzapine for the length of the chemotherapy cycle (typically five days), and two used it for a longer duration (eight weeks). Of the longer-duration studies, Nikbakhsh 2016 used it for participants receiving chemotherapy whilst Navari 2010b used it in participants not undergoing chemotherapy or radiotherapy. Instead Navari 2010b gave megestrol as a treatment for anorexia and cachexia as the standard treatment regimen, with the addition of olanzapine in the intervention arm.

We investigated the results for this comparison using subgroup analyses with respect to the dose of olanzapine. The results are presented at the end of the 'Effects of interventions' section.

\section{Absence of nausea or vomiting over the time period studied}

There is moderate-quality evidence that oral olanzapine increases the likelihood of being free from nausea or vomiting over the whole time period of the study when compared to placebo or no treatment (RR 1.98, 95\% Cl 1.59 to 2.47; participants = 561; studies $=3 ; I^{2}=0 \%$ ), or (OR $5.48,95 \% \mathrm{Cl} 1.35$ to $22.20 ; \mathrm{I}^{2}=85 \%$ ). The anticipated absolute effect was $24.6 \%$ more $(95 \% \mathrm{Cl} 14.8 \%$ more to $36.9 \%$ more), participants being free from nausea or vomiting. We calculated the number needed to treat for an additional beneficial outcome (NNTB), in order for one additional participant to be free from nausea and vomiting during the course of their treatment over standard therapy to be $5(95 \% \mathrm{Cl} 3.3-6.6)$, using an online calculator tool (GraphPad).

We downgraded the quality of this evidence to moderate due to inconsistency. The risk of nausea and vomiting in the control group varied from $19 \%$ to $40 \%$. This variation was probably due to the characteristics of the trials being different (such as type of cancer, chemotherapy treatment given, dose of olanzapine etc). We observed statistical heterogeneity when we assessed this using odds ratios $\left(I^{2}=85 \%\right)$, and therefore we used a random-effects model in this analysis. We identified a high risk of bias for one or two key domains for these studies. See Analysis 1.1.

One of these studies, Navari 2016a, reported absence of nausea overall and absence of vomiting overall as two separate outcomes. We chose to include the 'absence of nausea overall' data in the data contributing to our primary outcome of 'absence of nausea or vomiting over the time period studied'. This was because 
CINV is thought to be centrally mediated, rather than due to a mechanical cause leading to partial or complete obstruction of the gut. Nausea, therefore, most frequently precedes vomiting in participants with CINV. We acknowledge that this may slightly underestimate the incidence of nausea or vomiting in this study, should any participants have experienced vomiting unheralded by nausea, but recognise that this would be an infrequent occurrence. Additionally, when we added the data from Navari 2016a to the data from Mukhopadhyay 2016 and Lu 2013, the size of the effect reduced leading us to report a more conservative assessment of the efficacy of olanzapine in preventing overall nausea and vomiting rather than inflating it. This is because Mukhopadhyay 2016 and Lu 2013 reported relatively small numbers of participants when compared to Navari 2016a.

\section{Serious adverse events (extra pyramidal adverse events, prolonged QTc interval, neutropenia and agranulocytosis), as measured by the proportion of participants experiencing at least one of these events)}

We are uncertain whether olanzapine increases the likelihood of experiencing a serious adverse event when compared to placebo or no treatment $(\mathrm{RR} 2.46,95 \% \mathrm{Cl} 0.48$ to 12.55 ; participants = 889 ; studies $=7$ (Analysis 1.2), or OR $2.50,95 \% \mathrm{Cl} 0.48$ to 13.04), with an anticipated absolute difference of $0.7 \%$ more $(\mathrm{Cl} 0.2 \%$ fewer to $5.2 \%$ more; low-quality evidence). Of these seven studies, only one reported any serious adverse events (Navari 2016a). Five of these events were in the olanzapine arm. Two of these serious adverse events were haematological, although the exact nature of these was not stated. Three serious adverse events were not defined. Olanzapine is known to increase the risk of agranulocytosis, although the type of haematological abnormality found when monitoring the participants was not elucidated in the paper (Navari 2016a).

We downgraded the quality of this evidence to low due to imprecision and inconsistency. There was only a small number of events (5 in olanzapine, 2 in control arm), in one trial only and no events in either arm of six other trials, leading to wide confidence intervals. Additionally, we identified a high risk of bias for one domain for Mukhopadhyay 2016 and Mizukami 2014. We identified a high risk of bias for two domains for Lu 2013, Navari 2016a, and Zhang 2017, and we identified a high risk of bias in three domains for Navari 2013b and Zhao 2014. The proportion of information from trials at high risk of bias is sufficient to affect the interpretation of results.

\section{Participant's perception of treatment}

\section{Participant satisfaction measured by any scale, including Likert}

No studies reported participant satisfaction as an outcome measure.

\section{Participant preference}

One placebo-controlled RCT (Mizukami 2014), asked participants if they wished to use the drug they had been allocated to in the next chemotherapy cycle. In the olanzapine group, $83 \%(20 / 24)$, stated that they would like to use the drug compared to $58 \%$ (14/24), in the group receiving placebo (RR $1.43,95 \% \mathrm{Cl} 0.97$ to 2.09; participants $=48$; studies $=1$; low-quality evidence). See Analysis 1.3 .

We downgraded the quality of the evidence to moderate-quality due to imprecision. There was a very small sample size, 24 participants per arm, resulting in very large confidence intervals. We identified a high risk of bias in one key domain for this study.

\section{Quality of life}

Four studies assessed quality of life using different instruments. We felt it was not appropriate to attempt meta-analysis of these results as the domains measured by the scales were too heterogeneous. Three studies suggested that quality of life improved, although Nikbakhsh 2016 reported "no significant difference".

Mizukami $2014(\mathrm{~N}=48)$, reports that the "[olanzapine) group experienced a better QOL than the control group, as reported on the Functional Living Index-Emesis questionnaire $(P<0.0004)$ " and that "all patients in the (olanzapine) group indicated that CINV did not affect their daily activities (FLI-E score $<36 \mathrm{~mm}$ ), whereas $36 \%$ of the patients in the control group had their daily life activities affected by CINV".

Mukhopadhyay $2016(\mathrm{~N}=100)$, assessed global health status using the EEORTC QLQ C30 scale (Global Health Status range 2 to 14 , higher scores equal better quality of life). The global health status in the control group deteriorated from $10.08 \pm 0.43$ before treatment to $8.70 \pm 0.44 ; \mathrm{P}<0.0001$ after treatment, whereas the global health status improved in the olanzapine group (before $9.48 \pm 0.48$ and after treatment $9.60 \pm 0.47 ; \mathrm{P}=0.537$ ).

Navari 2010b ( $\mathrm{N}=80$ ), reported that $58 \%$ (23 of 39), of participants in the megestrol plus olanzapine arm had an "improved" quality of life at four and eight weeks compared to $13.5 \%$ (5/37), who received megestrol alone, on the FACT-G (version 3) scale. However, the criteria for a participant to be considered to have an 'improved' quality of life was not defined.

Nikbakhsh $2016(\mathrm{~N}=30)$, reported that "no significant difference was observed between the case and control groups about the patients QOL in ... total QOL score $(\mathrm{P}>0.05)$ " when olanzapine plus standard therapy was compared to standard therapy alone using the WHO-OOL-BREF scale.

Number of participants with Patient Global Impression of Change (PGIC), of much improved or very much improved (or equivalent wording)

No studies reported this outcome.

\section{Other adverse events}

We found low-quality evidence that olanzapine may increase the risk of other adverse events when compared to placebo or no treatment (RR 1.71, 95\% Cl 0.99 to 2.96; participants = 332; studies $\left.=4 ; I^{2}=0 \%\right)$. However, there is some uncertainty about this result. See Analysis 1.4.

We downgraded the quality of the evidence to low due to imprecision and inconsistency. There were wide confidence intervals and there was large variation in risks of adverse events between studies, with one study reporting an event rate of $23 \%$ in the placebo arm and $35 \%$ in the olanzapine arm. We identified a high risk of bias for one key domain for Mizukami 2014, two key domains for Zhang 2017, and three key domains for the remaining studies.

Zhang 2017 reported the highest rates of adverse events $(21 / 60$ in the olanzapine arm and $14 / 60$ in the control arm). In the olanzapine 
arm these included abdominal distension $(\mathrm{N}=6)$, dry mouth $(\mathrm{N}=$ $1)$, dizziness $(N=2)$, headache $(N=1)$, and drowsiness $(N=11)$. The reason for the high instance of adverse events in the intervention and control arms is not clear. Mizukami 2014 reported that one participant experienced drowsiness with olanzapine. Navari 2010b reported two participants who experienced mild sedation with olanzapine. Wang 2015 reported that one participant experienced "mild drowsiness" with olanzapine.

\section{Somnolence or fatigue}

We found moderate-quality evidence that the risk of participants experiencing somnolence or fatigue probably increases with olanzapine (RR 2.33, $95 \% \mathrm{Cl} 1.30$ to 4.18; participants $=464$; studies $\left.=5 ; I^{2}=17 \%\right)$, when compared to placebo or no treatment. See Analysis 1.5 .

We downgraded the quality of the evidence to moderate due to inconsistency. There was unexplained heterogeneity in the risk of somnolence between studies making it difficult to be confident that the true effect lies close to the estimate of the effect.

However, the reported incidence of somnolence in the olanzapine group varied greatly between studies from 1 out of $42(2.4 \%)$, to 13 out of $40(32.5 \%)$. The study with highest incidence of somnolence and fatigue (Zhao 2014), also administered a sedating antihistamine in its standard treatment regimen, which is known to interact with antipsychotic medication, exacerbating somnolence (Medicines.org).

When we excluded this study from the analysis, the risk of somnolence increased (RR 5.29, 95\% Cl 1.73 to 16.18; participants $=384$; studies $=4 ;\left.\right|^{2}=0 \%$ ).

\section{Withdrawals due to all causes}

We did not find evidence of a difference in the likelihood of withdrawal from studies between the olanzapine and no treatment/placebo groups although the overall withdrawal rate in both arms was low (RR 0.99, 95\% $\mathrm{Cl} 0.57$ to 1.73; participants = 943; studies $\left.=8 ; I^{2}=0 \%\right)$. See Analysis 1.6 .

\section{Withdrawals due to lack of efficacy}

Six RCTs (422 participants), reported that there were no withdrawals due to lack of efficacy in either arm. See Analysis 1.7.

\section{Withdrawals due to adverse events}

Of the six RCTs with data for withdrawals, only one RCT reported one withdrawal due to an adverse event in the olanzapine arm. No withdrawals due to adverse events were reported in the placebo/ no treatment arms in any study (RR $3.00,95 \% \mathrm{Cl} 0.13$ to 70.16 ; participants $=422$; studies $=6$ ). We are uncertain if olanzapine increases the risk of withdrawal due to an adverse event. See Analysis 1.8 .

\section{Withdrawals due to death}

Seven RCTs (542 participants), reported that there were no deaths during the study period.
$50 \%$ reduction in the severity of nausea or vomiting from baseline, as measured by a validated scale

No RCTs reported the number of participants with a $50 \%$ or greater reduction in the severity of nausea or vomiting from baseline, using a validated scale.

\section{Incidence of breakthrough nausea and vomiting and use of rescue antiemetics}

Olanzapine may reduce the need for breakthrough antiemetics (RR $0.38,95 \% \mathrm{Cl} 0.10$ to 1.47 ; participants $=501$; studies $=2 ; \mathrm{I}^{2}=54 \%$ ). Due to the high heterogeneity observed, we used a random-effects model for this analysis. See Analysis 1.9.

\section{No nausea or vomiting at different time points}

\section{No overall nausea or overall vomiting}

Olanzapine reduced nausea over the whole trial period compared to placebo or no treatment but the results are from only one study (RR 1.67, 95\% Cl 1.18 to 2.35; participants = 401; studies $=1$ ). See Analysis 1.10.

Olanzapine increased the likelihood of being free from vomiting over the whole trial period when we consider it alone compared to placebo or no treatment (RR $1.30,95 \% \mathrm{Cl} 1.12$ to 1.51 ; participants $=220$; studies $\left.=2 ;\left.\right|^{2}=0 \%\right)$. See Analysis 1.11 .

\section{No anticipatory (prior to chemotherapy), nausea or vomiting}

No trials reported anticipatory nausea or vomiting as an outcome.

No acute (within 24 hours of administration of chemotherapy), nausea or vomiting

We are uncertain if the likelihood of being free from acute nausea increases with olanzapine compared to no treatment/placebo (RR $1.37,95 \% \mathrm{Cl} 0.95$ to 1.98 ; participants $=585$; studies $=3 ; \mathrm{I}^{2}=89 \%$ ). See Analysis 1.12 .

We are uncertain if freedom from acute vomiting increases with olanzapine administration compared to placebo or no treatment when analysed using a random-effects model due to the high heterogeneity (RR $1.11,95 \% \mathrm{Cl} 0.97$ to 1.28 ; participants $=702$; studies $\left.=5 ; I^{2}=80 \%\right)$. See Analysis 1.13 .

We are uncertain if freedom from acute nausea and vomiting increases with olanzapine administration compared to placebo or no treatment when analysed using a random-effects model due to the high heterogeneity $(\mathrm{RR} 1.21,95 \% \mathrm{Cl} 0.86$ to 1.69 ; participants = 164 ; studies $\left.=2 ;\left.\right|^{2}=58 \%\right)$. See Analysis 1.14 .

No delayed (two to five days after administration of chemotherapy), nausea or vomiting

Olanzapine increased the likelihood of being free from delayed nausea in comparison to placebo or no treatment (RR 1.71, 95\% Cl 1.40 to 2.09; participants $=585$; studies $=3 ; I^{2}=0 \%$ ). See Analysis 1.15 .

Olanzapine increased the likelihood of being free from delayed vomiting in comparison to placebo or no treatment (RR 1.28, 95\% $\mathrm{Cl} 1.14$ to 1.42 ; participants $=702$; studies $=5 ; I^{2}=2 \%$ ). See Analysis 1.16 .

Olanzapine almost doubles the likelihood of being free from delayed nausea and vomiting compared to placebo or no treatment 
(RR 1.98, 95\% Cl 1.61 to 2.44; participants $=264$; studies $=3 ; I^{2}=$ 25\%). See Analysis 1.17 .

\section{Olanzapine versus NK1 antagonists}

Only one study (Shumway 2015), with 20 participants compared olanzapine with an NK1 antagonist (aprepitant), therefore we were unable to draw conclusions with certainty for this comparison.

\section{Absence of nausea or vomiting over the time period studied}

Shumway 2015 did not report this outcome.

Serious adverse events (extra pyramidal adverse events, prolonged QTc interval, neutropenia and agranulocytosis), as measured by the proportion of participants experiencing at least one of these events

The study reported serious adverse events in participants receiving olanzapine. We are uncertain whether this may favour the NK1 antagonist arm (RR $0.33,95 \% \mathrm{Cl} 0.02$ to 7.32 very low-quality evidence). See Analysis 2.1.

We downgraded the quality of evidence to very low due to imprecision and study limitations. There was only one study with a small sample size $(n=20)$, and very few events (one event in NK1 arm), leading to very imprecise results. The study states "for the first 2 cycles of chemotherapy", however, the length of each cycle was not defined.

\section{Participant's perception of treatment}

Shumway 2015 did not report participant satisfaction, participant preference, quality of life, or number of participants with Patient Global Impression of Change (PGIC), of much improved or very much improved.

\section{Other adverse events}

Shumway 2015 did not report this outcome.

\section{Somnolence or fatigue}

We are uncertain whether olanzapine increases somnolence and fatigue when compared to an NK1 antagonist (RR 1.33, 95\% Cl 0.40 to 4.49; participants $=20$; studies $=1$; very low-quality evidence). See Analysis 2.2.

We downgraded the evidence to very low quality due to imprecision and study limitations. There was only one study with a small sample size $(n=20)$, leading to very imprecise results. The study states "for the first 2 cycles of chemotherapy", however, the length of each cycle was not defined.

\section{Withdrawals due to all causes}

One participant in the olanzapine arm of Shumway 2015 lost their daily diary and therefore had to be withdrawn (RR 3.00, 95\% Cl 0.14 to 65.90; participants $=20$; studies $=1$ ). See Analysis 2.3.

There were no reported withdrawals due to lack of efficacy, adverse events or death.

\section{$50 \%$ reduction in the severity of nausea or vomiting from baseline, as measured by a validated scale}

Shumway 2015 did not report this outcome.
Incidence of breakthrough nausea and vomiting and use of rescue antiemetics

Shumway 2015 did not report this outcome.

\section{No nausea or vomiting at different time points}

We are uncertain if olanzapine is less effective than NK1 antagonists in preventing overall nausea ( $\mathrm{RR} 0.67,95 \% \mathrm{Cl} 0.14$ to 3.17 ; participants $=20$ ), due to data from a single study with a small sample size. See Analysis 2.7.

We are uncertain if olanzapine is less effective than NK1 antagonists in preventing acute nausea ( $\mathrm{RR} 0.60,95 \% \mathrm{Cl} 0.19$ to 1.86 ; participants $=20$; studies $=1$ ) (Analysis 2.8), or in preventing delayed nausea (RR 1.33, 95\% Cl 0.40 to 4.49; participants $=20$; studies $=1$ ) (Analysis 2.9), as there is insufficient evidence.

The study did not report the outcomes no vomiting, no anticipatory (prior to chemotherapy) nausea or vomiting, or no delayed (two to five days after administration of chemotherapy) nausea or vomiting.

\section{Olanzapine versus prokinetic}

Only one study compared olanzapine with prokinetic (metoclopramide), as treatment for breakthrough nausea and vomiting (Navari 2013b; 280 participants), therefore we were unable to draw conclusions with certainty for this comparison. All participants in both study groups also received background treatment including a 5-HT3 antagonist, NK1 antagonist and dexamethasone. Of the 280 participants, 112 had breakthrough CINV and received the treatment.

\section{Absence of nausea or vomiting over the time period studied}

Navari 2013b did not report this outcome.

Serious adverse events (extra pyramidal adverse events, prolonged QTc interval, neutropenia and agranulocytosis), as measured by the proportion of participants experiencing at least one of these events

Navari 2013b reported that no participants in either the olanzapine or the metoclopramide arm experienced any serious adverse event, including extra pyramidal features. See Analysis 3.1.

\section{Participant's perception of treatment}

Navari 2013b did not report participant satisfaction, participant preference, quality of life, or number of participants with Patient Global Impression of Change (PGIC), of much improved or very much improved.

\section{Other adverse events}

Navari 2013b did not report this outcome.

\section{Somnolence or fatigue}

Navari 2013b did not report this outcome.

\section{Withdrawals due to all causes}

It is uncertain whether olanzapine leads to a difference in withdrawal rates in the olanzapine arm over metoclopramide (RR $0.93,95 \% \mathrm{Cl} 0.14$ to 6.38 ; participants $=112$; studies $=1$ ). As only two 
participants withdrew from each arm the events rate is too low to assess with certainty. See Analysis 3.2.

\section{Withdrawals due to lack of efficacy}

One participant withdrew from the olanzapine arm and two from the metoclopramide arm due to lack of efficacy. We are uncertain whether this may favour the olanzapine arm (RR $0.47,95 \% \mathrm{Cl} 0.04$ to 4.99; participants $=112$; studies $=1$ ). See Analysis 3.3.

Withdrawals due to adverse events

No participants withdrew due to adverse events in either arm. See Analysis 3.4.

\section{Withdrawals due to death}

There were no withdrawals due to death were reported in either arm. See Analysis 3.5.

\section{$50 \%$ reduction in the severity of nausea or vomiting from baseline, as measured by a validated scale}

Navari 2013b did not report this outcome.

\section{Incidence of breakthrough nausea and vomiting and use of rescue antiemetics}

Navari 2013b did not report this outcome.

\section{No nausea or vomiting at different time points}

Navari 2013b did not report no anticipatory (prior to chemotherapy) nausea or vomiting, no acute (within 24 hours of administration of chemotherapy) nausea or vomiting, or no delayed (two to five days after administration of chemotherapy) nausea or vomiting.

\section{No overall nausea or overall vomiting}

Olanzapine may increase the likelihood of being free from nausea over the whole period of a study when compared with metoclopramide (RR 2.95, $95 \% \mathrm{Cl} 1.73$ to 5.02 ; participants $=112$; studies $=1$; Analysis 3.6), and of being free from vomiting (RR 3.03, $95 \% \mathrm{Cl} 1.78$ to 5.14 ; participants $=112$; studies $=1$ ). We assessed this study as being at low or unclear risk of bias for all key domains. See Analysis 3.7.

\section{Olanzapine versus 5-HT3 Antagonists}

Nakagaki 2017 ( $N=62$ ), was the only included RCT that compared olanzapine to 5-HT3 antagonists, therefore we were unable to draw conclusions with certainty for this comparison. This was a three-arm, parallel-group study that compared olanzapine to ondansetron and palonosetron. To avoid the risk of double counting the olanzapine data we decided to combine the two 5$\mathrm{HT} 3$ arm results rather than reporting the data as olanzapine versus palonosetron and olanzapine versus ondansetron.

\section{Absence of nausea or vomiting over the time period studied}

Nakagaki 2017 did not report this outcome.

Serious adverse events (extra pyramidal adverse events, prolonged QTC interval, neutropenia and agranulocytosis), as measured by the proportion of participants experiencing at least one of these events

Nakagaki 2017 reported no serious adverse events in either arm. See Analysis 4.1.

\section{Participant's perception of treatment}

Nakagaki 2017 did not report participant satisfaction, participant preference, quality of life, or number of participants with Patient Global Impression of Change (PGIC), of much improved or very much improved.

\section{Other adverse events}

Nakagaki 2017 did not report this outcome.

\section{Somnolence or fatigue}

Nakagaki 2017 did not report this outcome.

\section{Withdrawals due to all causes}

Nakagaki 2017 reported two withdrawals in the olanzapine arm (22 participants), and one in the combined 5-HT3 arm (44 participants), (RR 3.64, 95\% Cl 0.35 to 37.88; participants $=62$; studies $=1$ ). With so few events reported it is unclear whether there is a difference between the treatment groups. See Analysis 4.4.

\section{Withdrawals due to lack of efficacy}

The two withdrawals in the olanzapine arm were due to lack of efficacy compared with one in the 5-HT3 antagonists arm (RR 3.64, $95 \% \mathrm{Cl} 0.35$ to 37.88 ; participants $=62$; studies $=1$ ). With so few events reported it is unclear whether there is a difference between the treatment groups. See Analysis 4.5.

Nakagaki 2017 reported no withdrawals due to adverse events or death in either arm.

\section{$50 \%$ reduction in the severity of nausea or vomiting from baseline, as measured by a validated scale}

It is uncertain whether the use of olanzapine leads to more participants experiencing a $50 \%$ reduction in the severity of nausea or vomiting in the acute phase compared to $5-\mathrm{H} 3$ antagonists (RR 1.36, $95 \% \mathrm{Cl} 0.80$ to 2.34; participants $=62$; studies $=1$; Analysis 4.2). Olanzapine may have led to more participants with a $50 \%$ reduction in severity of nausea or vomiting in the delayed phase when assessed at 48 hours (RR 1.82, 95\% Cl 1.11 to 2.97; participants $=62$; studies $=1$; Analysis 4.3), compared to palonosetron and ondansetron.

\section{Incidence of breakthrough nausea and vomiting and use of rescue antiemetics}

Nakagaki 2017 did not report this outcome.

\section{No nausea or vomiting at different time points}

Nakagaki 2017 did not report overall, anticipatory (prior to chemotherapy), acute (within 24 hours of administration of chemotherapy), or delayed (two to five days after administration of chemotherapy) nausea or vomiting.

\section{Olanzapine versus dexamethasone}

Only one unblinded study (two reports, Liu/Tan 2015), with 229 participants, compared oral olanzapine $10 \mathrm{mg}$ daily for five days to 
$10 \mathrm{mg}$ of intravenous dexamethasone each day, on day 2 to day 5 , therefore we were unable to draw conclusions with certainty for this comparison.

\section{Absence of nausea or vomiting over the time period studied}

Liu/Tan 2015 did not report this outcome.

Serious adverse events (extra pyramidal adverse events, prolonged QTc interval, neutropenia and agranulocytosis), as measured by the proportion of participants experiencing at least one of these events

Liu/Tan 2015 reported no serious adverse events in either arm. See Analysis 5.1.

\section{Participant's perception of treatment}

Liu/Tan 2015 did not report participant satisfaction, participant preference, or Patient Global Impression of Change (PGIC).

\section{Quality of life}

Liu/Tan 2015 used the EORTC QLQ C30 version 3.0 scale to measure quality of life. They reported the Global Health Status domain (range: 0 to 100, higher scores equal better quality of life), and found that the change in Global Health Status score was greater for participants in the olanzapine group (improved by 3.25 points), than the dexamethasone group (decreased by 1.92 points). The study authors compared the results of the two groups using a $T$ test and reported the $P$ value as 0.005 , although they did not comment on the clinical significance of this change.

\section{Other adverse events}

Liu/Tan 2015 did not report this outcome.

\section{Somnolence or fatigue}

Liu/Tan 2015 report that 88 out of the 121 participants in the olanzapine arm experienced somnolence or fatigue but did not report the incidence of somnolence or fatigue in the comparison arm. It was therefore not possible to analyse these data.

\section{Withdrawals due to all causes}

The study did not report withdrawals due to lack of efficacy, adverse events or death in either arm (Liu/Tan 2015). See Analysis 5.2.

\section{$50 \%$ reduction in the severity of nausea or vomiting from} baseline, as measured by a validated scale

Liu/Tan 2015 did not report this outcome.

\section{Incidence of breakthrough nausea and vomiting and use of rescue antiemetics}

Liu/Tan 2015 did not report this outcome.

\section{No nausea or vomiting at different time points}

No overall nausea or overall vomiting

Olanzapine may increase the likelihood of being free from nausea (RR 1.73, 95\% Cl 1.37 to 2.18; participants $=229$; studies = 1 ; Analysis 5.3), and may slightly improve being free from vomiting (RR 1.27, $95 \% \mathrm{Cl} 1.10$ to 1.48 ; participants $=229$; studies $=1$; Analysis 5.4), over the whole period of the study when compared to dexamethasone.
No anticipatory (prior to chemotherapy), nausea or vomiting Liu/Tan 2015 did not report this outcome.

No acute (within 24 hours of administration of chemotherapy), nausea or vomiting

Olanzapine may lead to little or no difference in rates of acute nausea (RR 1.07, 95\% Cl 0.99 to 1.14; participants = 229; studies $=1$; Analysis 5.5), or acute vomiting (RR $1.01,95 \% \mathrm{Cl} 0.94$ to 1.08 ; participants $=229$; studies $=1$; Analysis 5.6 ), when compared to dexamethasone.

No delayed (two to five days after administration of chemotherapy), nausea or vomiting

Olanzapine may slightly improve the likelihood of being free from delayed nausea (RR 1.66, 95\% Cl 1.33 to 2.08; participants = 229; studies $=1$; Analysis 5.7), and delayed vomiting (RR 1.25, 95\% Cl 1.07 to 1.45 ; participants $=229$; studies $=1$; Analysis 5.8), when compared to dexamethasone.

\section{Subgroup analyses}

We were only able to complete subgroup analysis for the comparison of olanzapine versus placebo or no treatment, as this was the only comparison with sufficient data. Methods for the subgroup are presented in the methods section: Subgroup analysis and investigation of heterogeneity.

\section{Effect of dose on efficacy and adverse events}

We assessed the effect of dose $(2.5 \mathrm{mg}$ versus $5 \mathrm{mg}$ versus $10 \mathrm{mg}$ twice daily), on efficacy and adverse events. This was to establish whether there could be a dose response effect of olanzapine in the context of nausea and vomiting, in order to identify the lowest effective dose and the dose associated with the fewest adverse events.

\section{Doses analysed}

There were sufficient data to undertake subgroup analysis to assess $5 \mathrm{mg}$ and $10 \mathrm{mg}$ daily doses. Some studies gave these as daily doses, and others as divided doses (i.e. $2.5 \mathrm{mg}$ twice a day or $5 \mathrm{mg}$ twice a day). Although one study (Nikbakhsh 2016), used $2.5 \mathrm{mg}$ daily the data were not presented in a way that we could extract only the effect of the $2.5 \mathrm{mg}$ dose.

\section{Efficacy}

\section{No nausea or vomiting over the trial period}

The subgroup analysis results found that, compared to placebo/ no treatment the $5 \mathrm{mg}$ and $10 \mathrm{mg}$ dose may have similar efficacies in preventing nausea and vomiting over the whole trial period. The test for subgroup differences did not indicate that there were differences between the groups $\left(P=0.86 ; I^{2}=0 \%\right)$. Analysis 6.1.

- 5 mg olanzapine: RR 2.00, 95\% Cl 1.19 to 3.36; participants = 60; studies $=1$

- 10 mg olanzapine: $\mathrm{RR} 1.98,95 \% \mathrm{Cl} 1.40$ to 2.80 ; participants = $501 ;$ studies $=2 ; 1^{2}=50 \%$ 


\section{Adverse events}

\section{Serious adverse events}

$5 \mathrm{mg}$ olanzapine: none of the five studies included $(\mathrm{N}=388$ ) reported any serious adverse events in either the olanzapine or placebo/no treatment arm.

$10 \mathrm{mg}$ olanzapine: two studies $(\mathrm{N}=501)$ reported serious adverse events. Only one study found any adverse events and reported five serious adverse events in the olanzapine arm and two in the comparison arm (RR 2.46, 95\% Cl 0.48 to 12.55 ; participants = 501 ; studies $=2$; Analysis 6.2). The nature of all seven serious adverse events was not described, however, the paper stated that two of the serious adverse events in the olanzapine arm were "haematologic". Olanzapine is known to increase the risk of agranulocytosis, although the type of haematological abnormality found when monitoring the participants was not elucidated in the paper (Navari 2016a).

Whilst this might represent an increased risk of serious adverse events with the $10 \mathrm{mg}$ daily dose of olanzapine, this evidence is of low quality and when considering it is a subgroup analysis is not advisable to take this as a causal link.

\section{Other adverse events}

We are uncertain whether other adverse events are increased more with a $5 \mathrm{mg}$ olanzapine dose (RR 4.00, 95\% Cl 0.46 to 34.85 ; participants $=128 ;$ studies $\left.=2 ; I^{2}=0 \%\right)$, compared with a $10 \mathrm{mg}$ olanzapine dose (RR 1.55, 95\% Cl 0.88 to 2.73; participants = 204; studies $=2 ; 1^{2}=0 \%$; Analysis 6.3). The test for subgroup differences did not indicate there were significant differences between the groups $\left(P=0.41, I^{2}=0 \%\right)$.

\section{Somnolence or fatigue}

The use of $10 \mathrm{mg}$ olanzapine may be associated with an increased risk of somnolence or fatigue (RR $5.33,95 \% \mathrm{Cl} 1.60$ to 17.81 ; participants $=304$; studies $=3 ; \mathrm{I}^{2}=0 \%$, when compared to the $5 \mathrm{mg}$ dose (RR $1.48,95 \% \mathrm{Cl} 0.75$ to 2.91 ; participants $=160$; studies $=2$; $\mathrm{I}^{2}=0 \%$ ). However, the test for subgroup differences indicates that there is no significant difference between the two groups $(P=0.07$; $\mathrm{I}^{2}=69.8 \%$; Analysis 6.4).

\section{Serious adverse events when administered for longer than five days}

It should be noted that, apart from two RCTs (Navari 2010b; Nikbakhsh 2016), the data identified in this review only describe the short-term administration of olanzapine for nausea and vomiting, usually up to a maximum of five days' duration. Navari 2010b (N $=80$ ) administered $5 \mathrm{mg}$ olanzapine daily for eight weeks and reported no serious adverse events. Nikbakhsh $2016(\mathrm{~N}=30)$ used olanzapine doses ranging from $2.5 \mathrm{mg}$ to $10 \mathrm{mg}$ daily, for eight weeks, according to individual participant tolerance. The study did not report any adverse events, serious or otherwise.

\section{Summary}

From the pre-specified but observational subgroup analysis, there is an indication that the $5 \mathrm{mg}$ dose might achieve as good an antiemetic effect as the $10 \mathrm{mg}$ dose with less somnolence and fatigue. It is uncertain whether there is an increased risk of serious or other adverse events with $10 \mathrm{mg}$ compared to $5 \mathrm{mg}$ of olanzapine.

\section{Other planned subgroups}

There was insufficient evidence to undertake the planned subgroup analysis to assess the impact of the different clinical settings (chemotherapy, radiotherapy, chemoradiotherapy or no active cancer treatment), on the efficacy of olanzapine. Equally, there was insufficient evidence to undertake the planned subgroup analysis to assess the impact of MEC or HEC, on its efficacy.

\section{DISCUSSION}

\section{Summary of main results}

We included 14 RCTs (1917 participants), from high-, middleand low-income countries, representing over 24 different cancers. Nine studies compared olanzapine with placebo/no treatment for the prevention of CINV (Summary of findings for the main comparison). All participants also received standard antiemetic treatment. Four RCTs each compared olanzapine with one of the following active comparisons: NK1 antagonists (participants $=20$ ), 5-HT3 antagonists (participants $=62$ ), prokinetics (participants $=$ 112 ), and dexamethasone (participants $=229$ ). The comparison against NK1 antagonists is presented in Summary of findings 2 . We included a further RCT in our qualitative synthesis but this study did not contribute data to the quantitative analysis (Clemons 2016).

\section{Efficacy}

\section{Absence of nausea or vomiting over the whole time period studies}

Three out of nine studies contributed data to our primary outcome of 'Absence of nausea or vomiting over the time period studied'. There is moderate-quality evidence that olanzapine probably almost doubles the likelihood of being free from nausea and vomiting over the trial period when compared to placebo or no treatment.

The primary efficacy outcome of no nausea or vomiting over the trial period was not reported in any of the trials comparing olanzapine against an active treatment.

\section{Efficacy for no nausea or no vomiting over different time periods}

\section{Efficacy for the overall period of the study}

When compared against no treatment or placebo olanzapine probably increases the likelihood of not having nausea or not having vomiting over the whole time period studied. When olanzapine was compared against prokinetics and dexamethasone, there may have been a slight reduction in nausea and a slight reduction in vomiting. No other comparisons reported this outcome.

\section{Efficacy for the acute phase of the study}

Compared to no treatment or placebo it is uncertain whether olanzapine impacts greatly on acute nausea or acute vomiting. When compared against active comparators, there was little or no difference between acute nausea versus NK1 antagonists and dexamethasone. No other comparisons reported this outcome.

\section{Efficacy for the delayed phase of the study}

When compared to no treatment or placebo, olanzapine probably increases the likelihood of freedom from delayed nausea and delayed vomiting. There was little or no difference in delayed 
nausea when olanzapine was compared against NK1 antagonists but there may have been a slight reduction in both nausea and vomiting in the delayed phase with olanzapine compared against dexamethasone. No other comparisons reported this outcome.

The trial comparing olanzapine with 5-HT3 antagonists reported that, although there was little or no difference in the percentage of participants with a $50 \%$ improvement in nausea at 24 hours, they may have a slight improvement with olanzapine at 48 hours.

Whilst olanzapine almost doubles the likelihood of being free from nausea and vomiting overall and in the delayed phase, it does not appear to impact greatly, if at all, acute nausea and vomiting when compared to placebo/no treatment, NK1 antagonists or dexamethasone. The studies that compared olanzapine to prokinetics or 5-HT3 antagonists did not report incidence of acute nausea and vomiting. The reason for its delayed onset of antiemetic action is not clear from our analysis. It is possible that this may be related to when olanzapine achieves steady state as its "halflife ranges from 21 to 54 hours (5th to 95th percentile; mean of 30 hr)" (FDA).

\section{Adverse events}

\section{Serious adverse events with oral olanzapine}

It is uncertain whether olanzapine increases the risk of serious adverse events compared with no treatment or placebo, as only one trial out of seven reported any serious adverse events. Postmarketing adverse events surveillance may be more reflective of the true incidence of these rare events.

\section{Serious adverse events with long-term oral olanzapine}

Only two RCTs administered olanzapine for nausea and vomiting, for more than five days (Navari 2010b; Nikbakhsh 2016). Navari 2010b administered $5 \mathrm{mg}$ daily for eight weeks and reported no serious adverse events. Nikbakhsh 2016 used doses ranging from $2.5 \mathrm{mg}$ to $10 \mathrm{mg}$ daily for eight weeks, according to individual participant tolerance, but provided no report of adverse event data, serious or otherwise. We are therefore very uncertain as to the risks of serious adverse events when olanzapine is administered for longer than five days. Whilst the only frequently identified adverse event across all the included trials was somnolence, and serious adverse effects were infrequently identified, RCTs are not the best way of identifying adverse events, particularly those that occur infrequently. In view of the paucity of data relating to long-term administration of olanzapine, clinicians should be aware of and monitor for adverse events, including extra pyramidal features, prolonged QTC interval, neutropenia and agranulocytosis, and diabetes mellitus if olanzapine is administered for longer than five days (Anonymous 2017).

\section{Somnolence and fatigue}

There is moderate-quality evidence that olanzapine increases the risk of somnolence and fatigue, although this may be partially attenuated when $5 \mathrm{mg}$ of olanzapine daily is administered compared to $10 \mathrm{mg}$ daily.

\section{Quality of life}

There is a suggestion that olanzapine may slightly improve overall quality of life, or prevent the reduction in quality of life seen in the control groups when chemotherapy is administered, although it was not possible to analyse these data as studies used a wide range of scores to assess them and the reporting was poor.

\section{Overall completeness and applicability of evidence}

\section{Evidence gaps}

Additional published data and studies are required to assess the impact of $2.5 \mathrm{mg}, 5 \mathrm{mg}$ and $10 \mathrm{mg}$ doses on the efficacy and safety of olanzapine.

There was only one trial that studied participants who were not receiving active anticancer treatment with chemotherapy, radiotherapy or chemoradiotherapy (Navari 2010b). One trial included participants receiving chemoradiotherapy (Nakagaki 2017), and there was none where participants only received radiotherapy. This limits the applicability of the results of this review in participants receiving radiotherapy alone predominantly and with less certainty to those receiving no active treatment. Further research is required in these populations.

There was only one study that compared olanzapine head to head against an NK1 antagonist and this study was underpowered with only 10 participants per arm (Shumway 2015), therefore we believe that further research is needed to assess whether olanzapine is superior, non-inferior or inferior to NK1 antagonists.

No RCT followed participants receiving olanzapine for more than eight weeks. Longer term, larger studies are needed to improve our understanding of the risk of serious adverse events (particularly extrapyramidal side effects, hyperglycaemia, agranulocytosis and QTc prolongation), when participants require olanzapine over longer time periods.

Finally, despite searching for RCTs that administered olanzapine by any route, we found only studies that used the oral route. Further studies are needed to evaluate the administration of olanzapine via non-oral routes, as this lack of evidence currently limits its clinical use as an antiemetic.

\section{Palliation of other important clinical symptoms}

\section{Anxiety and depression}

Nikbakhsh 2016 assessed anxiety and depression on the validated HADS, over the participants' course of chemotherapy. They reported that anxiety and depression increased in the control group with the administration of chemotherapy. In the olanzapine group they noted an improvement in both anxiety and depression.

\section{Anorexia and cachexia}

Three of the included RCTs reported an improvement in anorexia and cachexia in the olanzapine compared to the control group (Mizukami 2014; Navari 2010b; Nikbakhsh 2016).

Further systematic reviews are therefore needed to establish the role of olanzapine in the palliation of cachexia, anxiety, depression and delirium.

\section{Serious adverse events and common drug interactions when olanzapine is injected}

The review authors noted that, whilst we could not identify any RCTs that reported using an injectable form of olanzapine, these have been reported in case series. These reports note that 
respiratory depression occurs when olanzapine is administered via the intravenous route in doses of $1.25 \mathrm{mg}, 2.5 \mathrm{mg}$ and $5 \mathrm{mg}$. Severe respiratory depression leading to hypoxia requiring airway stimulation, repositioning or intubation has been reported (Martel 2016). In light of these serious safety concerns and in the absence of any RCTs in which the intravenous route or any other injectable route has been used, we caution against extrapolation of the oral data to the intravenous route, as there is currently insufficient data to support the use of intravenous olanzapine.

Additionally, it is important to note that if olanzapine is injected intramuscularly, there is also known to be a risk of cardiovascular and respiratory depression. It is advised that blood pressure, heart rate and respiratory rate are monitored for a minimum of four hours following intramuscular administration and that at least one hour has elapsed following intramuscular administration before parenteral benzodiazepines are administered (BNF). This is of particular relevance to Palliative Medicine Specialists, as is the caution in patients with ileus (BNF).

\section{Quality of the evidence}

There is moderate-quality evidence that olanzapine probably improves nausea and vomiting when compared to placebo or no control, but probably leads to somnolence and fatigue. However, the quality of evidence for all other outcomes was low or very low. We therefore cannot interpret these results of these outcomes with certainty and further research is needed to address these.

Whilst there is moderate-quality evidence to support olanzapine in CINV when added to standard antiemetic therapy, further research is needed to establish olanzapine's role in chemoradiotherapy, radiotherapy and in participants not receiving active anticancer treatment.

Downgrading of evidence was primarily for the imprecision of the results due to the small sample sizes and low event rates (adverse events). There were also large variations between some of the trials in the base rates of some outcomes. This may have been due to different doses of olanzapine, different population characteristics and cancer treatments, or differences in the standard adjuvant antiemetic regimens between trials. The individual trials were generally judged to be at an 'unclear' or 'low' risk of bias. Only six of the studies presented clear information on randomisation, and only three had clear data on allocation concealment. This is perhaps surprising given the age of the studies.

\section{Potential biases in the review process}

\section{Publication bias}

We did not detect any serious publication bias and there were insufficient studies in each comparison to produce funnel plots for any outcome.

We could only adequately assess full-text RCTs, abstracts where additional information was provided by the study author, or unpublished trials where the full results were made available to us. We therefore excluded four RCTs that had only been published as abstracts, despite requesting additional information from the study authors. We identified two terminated unpublished RCTs and one completed unpublished RCT CTRCC-14004093, for which we were unable to obtain results.
One RCT was unobtainable (Wang 2012). Lu 2013 and Zhao 2014 were translated from Chinese and data extracted by one translator. Additionally, full texts in German, Dutch and other Chinese language papers were translated and reviewed at the full-text screening stage before exclusion. Given the number of foreign language studies identified, a search of the foreign language databases may have identified more studies.

Additionally, given the high number of studies that are ongoing or yet to be published as full-text articles, it will be important to update this review when these become available.

\section{Potential biases within the data analysis}

As discussed above, we elected to include the data from Navari 2016a for 'overall absence of nausea' in our analysis of 'overall nausea and vomiting' in an attempt to reduce the risk of overestimating the relative risk of being free from nausea and vomiting over the whole trial period that is present when only data from studies with small sample size is considered. This resulted in a reduction in the size of anticipated absolute effect and the size of relative risk reported.

It is important to remain vigilant for these, as RCTs are not the most effective way of monitoring adverse events, particularly serious but rare adverse events, such as extra pyramidal adverse events, prolonged QTC interval, neutropenia and agranulocytosis, and diabetes mellitus, which are likely to be underreported in RCT data due to the small numbers of participants and short trial duration.

Instead of reporting overall absence of nausea and vomiting, most studies reported 'complete response' as their primary outcome. This was most frequently defined as no vomiting or use of breakthrough antiemetic medications over the course of the trial. We acknowledge that this outcome is likely to be more easily measured as it removes any concern over the possible subjectivity of nausea assessment and self-reporting, as any breakthrough medication would lead to a documented administration. However, the review authors were concerned that some participants might have felt nauseous but not received breakthrough medication for any number of reasons, whilst still being documented as having completely responded to the intervention. We were concerned that using this surrogate outcome might lead to an over estimation of the efficacy of olanzapine, when in fact, participants had experienced nausea but had not been treated with breakthrough medication. We felt that being entirely free from nausea or vomiting would be of greater importance to patients than whether their nausea reached a threshold severe enough to require breakthrough medication and whether they were able to receive the medication. We considered that freedom from nausea and vomiting was a 'hard' outcome, meaning that we were less likely to overestimate the efficacy of olanzapine in our analysis.

\section{Agreements and disagreements with other studies or reviews}

\section{Compared to Cochrane Reviews of the antiemetic efficacy of antipsychotics}

Two other antipsychotics are frequently used as antiemetics.

Haloperidol is a dopamine receptor antagonist. It is administered via the oral, subcutaneous, intravenous or intramuscular route and may be administered via the intranasal route. Murray-Brown 2015 
found only one RCT of 22 participants, who were given haloperidol as a gel on the wrist, which is "reported not to be absorbed by this route" and concluding that there was insufficient RCT evidence to determine its effectiveness in their Cochrane systematic review.

Levomepromazine is another antipsychotic that is currently used as an antiemetic. As with olanzapine, it is known to act on a range of neurotransmitter receptors including dopaminergic, cholinergic, serotonin and histamine. Unfortunately, Cox 2015 found no eligible RCTs in their Cochrane systematic review.

This is, therefore, the first Cochrane systematic review to find RCT-level evidence to support the theory that an antipsychotic medication improves nausea and vomiting in participants with a cancer diagnosis.

\section{Compared to other systematic reviews and meta-analyses}

The findings of this Cochrane systematic review of olanzapine for the prevention and treatment of nausea and vomiting in adults are in keeping with all nine independent systematic reviews and metaanalyses on the topic.

- Wang 2014 found that olanzapine increased the odds of being free from nausea and vomiting in both the overall (OR 1.95, Cl 1.17 to $3.23 ; \mathrm{P}=0.01$ ), and delayed phases (OR 2.65, $\mathrm{Cl} 1.36$ to $5.15, P=0.004)$.

- Kumar 2017 reported that "olanzapine was statistically superior for five primary endpoints except for no nausea in acute period. In the non-steroids cohort, olanzapine was superior for no emesis in all three periods but statistically significant only for delayed period."

- Hocking 2014 found that "regimens including olanzapine were associated with significant improvements in CINV prevention with both HEC and MEC."

- In a narrative systematic review van der Vorst 2015 reported that "olanzapine...show[ed) highly effective complete response (CR), rates."

- Equally, Yoodee 2017 concluded that "olanzapine is effective and safe at reducing during the delayed and overall phase of the CINV prevention".

- Recently, Yang 2017 also concluded that "olanzapine is an excellent alternative for the prophylaxis of CINV. Olanzapine 5 $\mathrm{mg}$ per day should be recommended as the initial dose because of equivalent efficacy to a $10 \mathrm{mg}$ dose but a lower potential risk of side effects. Further studies are needed to explore the optimal combination of medicines."

- Chiu 2016a again found that "olanzapine is more efficacious than other standard antiemetics for the rescue of CINV and its inclusion improves control in the prevention setting. Given the possible reduction in side effects, the use of a 5-mg dose of olanzapine should be considered. Future RCTs should compare the 5-mg versus the 10-mg dosages further and report on the efficacy and percentage of patients developing side effects."

- Chow 2016 analysed the data from phase one and phase two clinical trials of olanzapine as an antiemetic. They concluded that "olanzapine is efficacious and safe when used as a prophylaxis for CINV."

- Chelkeba 2017 summarised the results of their meta-analysis by concluding that "the bottom line is that olanzapine containing regimen is statistically superior to non-olanzapine regimen in preventing CINV in all endpoints and phases."

\section{Compared to cost-effectiveness studies}

Chanthawong 2017 performed a cost-effectiveness analysis across four countries in South East Asia. They used "a decision tree model, clinical and economic outcomes" evaluate management of CINV following HEC. The study found that "the addition of olanzapine is cost-effective and viable to prevent CINV" in Thailand, Malaysia, Indonesia, and Singapore when olanzapine was added to standard doublet (dexamethasone and ondansetron), and triplet (dexamethasone and palonosetron and aprepitant), antiemetic therapy.

In the UK in 2017 the cost of a five-day course of $5 \mathrm{mg}$ orodispersible olanzapine to prevent CINV during a standard cycle of chemotherapy is GBP 7.30. This can be compared to the cost of a three-day course of aprepitant oral capsules at GBP 100.64 or a course of a single intravenous injection ampoule of fosaprepitant at GBP 47.42, with the additional nursing cost of intravenous administration (BNF). However, it is yet to be established whether olanzapine is superior, non-inferior or inferior to NK1 antagonists.

\section{AUTHORS' CONCLUSIONS}

\section{Implications for practice}

\section{For adults with cancer-related nausea and vomiting}

Olanzapine probably reduces the number of people with cancer who experience chemotherapy-induced nausea and vomiting (CINV), when given by mouth in addition to a standard antisickness treatment, compared to the standard antisickness treatment alone. Olanzapine probably almost doubles the likelihood that there will be no nausea or vomiting during a course of chemotherapy. The evidence we reviewed found that with standard antisickness treatment about $75 \%$ of participants experienced some nausea and may have vomited during chemotherapy. When olanzapine was added to standard antisickness treatment this fell to $50 \%$.

Unfortunately, olanzapine probably increases somnolence. This may be more likely with $10 \mathrm{mg}$ a day than $5 \mathrm{mg}$. The risk of other adverse events may be increased by olanzapine when used with other antisickness treatment compared to using the antisickness treatment alone.

Olanzapine is unlicensed for this indication.

\section{For clinicians}

Oral olanzapine is probably an effective antiemetic when used with a standard antiemetic regimen compared to the standard antiemetic regiment alone in the treatment of people experiencing CINV with solid tumours. Whilst $5 \mathrm{mg}$ may have a similar antiemetic effect to $10 \mathrm{mg}$, we did not include studies directly comparing doses in this review. There is a lack of randomised controlled trial (RCT), evidence to support the use of $2.5 \mathrm{mg}$ olanzapine as an antiemetic or to support the use of olanzapine administration by any route other than oral. There may be an increase in adverse events with olanzapine, and somnolence is probably more likely and may limit its clinical use, although $5 \mathrm{mg}$ may cause less somnolence than $10 \mathrm{mg}$. We are less certain about the risk of serious adverse events, particularly hyperglycaemia, agranulocytosis, QTc prolongation and extra-pyramidal adverse events.

There is limited information regarding the efficacy and safety of olanzapine compared to other antiemetics. 
Safety concerns have been raised in the literature that intravenous olanzapine may lead to serious respiratory depression. Cardiovascular and respiratory depression have also been noted when it is administered intramuscularly. The results of this review therefore cannot be extrapolated to support the use of olanzapine via the intravenous, intramuscular or subcutaneous route.

Olanzapine is currently listed as an antipsychotic, however, it is not licensed for the prevention or treatment of nausea and vomiting in the UK by the Medicines and Healthcare products Regulatory Agency (MHRA), or in the USA by the Food and Drug Administration (FDA). Its use as an antiemetic is therefore currently an off-licence use in the UK and USA.

\section{For policy makers}

Oral olanzapine is probably an effective antiemetic when added to standard treatment regimens for adults with CINV. Olanzapine is a low-cost, once-daily antiemetic that is available as an oral dispersible wafer, with infrequent adverse events. Olanzapine is currently listed as an antipsychotic, however, it is not licensed for the prevention or treatment of nausea and vomiting in the UK by the MHRA, or in the USA by the FDA. Its use as an antiemetic is therefore currently an off-licence use in the UK and USA.

Additionally, there remains limited evidence for use of olanzapine instead of other antiemetics. In particular there is insufficient evidence to support its use in preference to NK1 antagonists for the treatment of chemotherapy related nausea and vomiting.

\section{For funders}

There is moderate-quality evidence that oral olanzapine is an effective antiemetic in CINV. Further research is urgently needed to assess the optimal dose regimen. Additionally, further research is needed to assess the relative efficacies of olanzapine and NK1 antagonists, as olanzapine may be a more cost-effective option. Studies are also needed to look at which settings it is most effective in, particularly in patients receiving radiotherapy or no active anticancer treatment, to address the current paucity of evidence in these groups.

Future trials should be adequately powered to detect a difference and should have an explicit method for reporting adverse effects, including serious adverse events and somnolence.

\section{Implications for research}

\section{General implications}

Large, high-quality RCTs, with patient-centred outcomes are needed in order to establish the optimal effective dose regimen of olanzapine that achieves the greatest antiemetic effect with the least incidence of adverse events, particularly somnolence. We await the outcomes of the 13 ongoing studies, and the 8 studies awaiting classification, with interest.

One published abstract (Hashimoto 2016), one unpublished study (Yanai 2015), and two ongoing studies (Mukhopadhyay 2017a; Nagashima et al 2015), address $10 \mathrm{mg}$ olanzapine compared to 5 $\mathrm{mg}$. The review authors are not aware of any studies exploring the efficacy of $2.5 \mathrm{mg}$ olanzapine as an antiemetic.

\section{Design}

In future RCTs, investigators should ensure that the dosing regimen of the standard therapy is the same in both arms so that any observed effect is attributable to the intervention of interest, olanzapine, rather than its combined effect with the variation in the standard therapy administered. In particular, the dose of dexamethasone, which is known to be an effect modifier, should be the same in the intervention and comparator arms. However, it is also important that the regimen of NK1 antagonist or 5-HT3 antagonist are identical in each comparison arm.

RCTs with a longer intervention and follow-up period are needed to establish olanzapine's efficacy and safety when used for more than five days.

The parenteral administration of olanzapine is yet to be studied in an RCT. This is of clinical importance as inherent in vomiting is the risk of failure to ingest and absorb it when the oral route is used, although counterbalanced by the known serious respiratory and cardiovascular adverse events associated with the intravenous and intramuscular routes.

Further research is needed to establish its non-inferiority or otherwise when compared to other antiemetics currently used, including NK1 antagonists, 5-HT3 antagonists, prokinetics, steroids, and when compared directly to current standard care. We await the full published results of Nguyen 2017, an RCT comparing olanzapine and omeprazole directly to standard care and omeprazole without any other standard therapy. The published abstract states that olanzapine $10 \mathrm{mg}$ oral and omeprazole 20 $\mathrm{mg}$, once daily for three days is "simple, cheap cost, safe, and effective in preventing vomiting, reducing nausea, and preserving QoL [quality of life)" when compared to dexamethasone $4 \mathrm{mg}$ twice daily, omeprazole $20 \mathrm{mg}$ twice daily and metoclopramide $20 \mathrm{mg}$ three times daily, all for five days.

\section{Measurement}

We encourage investigators to use the 'hard' outcome of 'no nausea, no vomiting' rather than the surrogate outcome 'no vomiting, no breakthrough medication' when reporting primary outcomes. When reporting serious adverse events it is important that the nature of these are fully defined. Additionally, if consensus were reached in the future to enable one quality-of-life measure to be universally adopted this would allow comparison of data across studies more easily, and therefore aid the applicability of this data in the clinical setting.

\section{Other}

Studies are urgently needed to explore impact of the different anticancer treatment on the efficacy and safety of olanzapine during different phases of illness, particularly in radiotherapy, chemoradiotherapy or no active cancer treatment. We are aware of two ongoing studies (JPRN-UMIN000010317 and NCT03137121), addressing the use of olanzapine in participants with advanced cancer who are not receiving any active anticancer therapy. In JPRN-UMIN000010317 participants have malignant bowel obstruction. 


\section{ACKN OWLEDGEMENTS}

AS was a Cochrane UK Fellow, funded by Health Education Thames Valley (HETV), during the period the systematic review was undertaken.

Cochrane Review Group funding acknowledgement: this project was supported by the National Institute for Health Research, via Cochrane Infrastructure funding to Cochrane Pain, Palliative and Supportive $\mathrm{Care}(\mathrm{PaPaS})$. The views and opinions expressed therein are those of the authors and do not necessarily reflect those of the Systematic Reviews Programme, NIHR, NHS or the Department of Health.
We would like to thank Sheena Derry and Phil Wiffen for their advice and support throughout the process of developing the title and protocol.

We would also like to thank Anne Eisinga (Cochrane UK), for her advice and assistance throughout the review process and particularly for assisting translating a Dutch language paper.

We would like to thank Aleksandra Brett, Robert Thomson, Judith Ames and Eli Harriss for the time and energy they dedicated to tirelessly searching for papers at the Knowledge Centre, Bodleian Health Care Libraries, University of Oxford, without whom this systematic review would not have been possible.

We would like to thank Shanshan Wu (China), Yu-Tian Xiao (China), and Aidan Tan (USA), for assisting by translating and data extracting Chinese language papers. 


\section{R E F E R E N C E S}

\section{References to studies included in this review}

\section{Clemons 2016 \{published data only\}}

Clemons M, Bouganim N, Smith S, Mazzarello S, Vandermeer L, Segal R, et al. Risk model-guided antiemetic prophylaxis vs physician's choice in patients receiving chemotherapy for earlystage breast cancer: a randomized clinical trial. JAMA Oncology 2016;2(2):225-31.

Dranitsaris G, Mazzarello S, Smith S, Vandermeer L, Bouganim N, Clemons M. Measuring the impact of guidelinebased antiemetic therapy on nausea and vomiting control in breast cancer patients with multiple risk factors. Supportive Care in Cancer 2016;24(4):1563-9.

\section{Liu/Tan 2015 \{published data only\}}

* Liu J, Tan L, Zhang H, Li H, Liu X, Yan Z, et al. QoL evaluation of olanzapine for chemotherapy-induced nausea and vomiting comparing with 5-HT3 receptor antagonist. European Journal of Cancer Care 2015;24(3):436-43.

Tan L, Liu J, Liu X, Chen J, Yan Z, Yang H, et al. Clinical research of olanzapine for prevention of chemotherapy-induced nausea and vomiting. Journal of Experimental and Clinical Cancer Research 2009; Vol. 28:131.

\section{Lu 2013 \{published data only\}}

Lu Y L, Liu W, Du Y J, Feng L, Wang Y D, Wang L. Antiemetic effect of low dose olanzapine in solid tumor chemotherapy. Chinese Journal of Cancer Prevention and Treatment 2013;20(7):544-54.

\section{Mizukami 2014 \{published data only\}}

Mizukami N, Yamauchi M, Koike K, Watanabe A, Ichihara K, Masumori $\mathrm{N}$, et al. Olanzapine for the prevention of chemotherapy-induced nausea and vomiting in patients receiving highly or moderately emetogenic chemotherapy: a randomized, double-blind, placebo-controlled study. Journal of Pain and Symptom Management 2014;47(3):542-50.

Mukhopadhyay 2016 \{published and unpublished data\}

* Mukhopadhyay S, Kwatra G, Alice K, Badyal D. Role of olanzapine in chemotherapy-induced nausea and vomiting on platinum-based chemotherapy patients: a randomized controlled study. Supportive Care in Cancer 2017;25(1):145-54.

Mukhopadhyay S, Kwatra G, Alice P, Badyal D. Role of olanzapine in chemotherapy-induced nausea and vomiting on platinum-based chemotherapy patients: a randomized controlled study. Supportive Care in Cancer 2016;25:1-10.

Mukhopadhyay S, Kwatra G, Badyal D, Kingsley P. Olanzapine, concurrent chemo-radiation patients and chemotherapy induced nausea and vomiting: the surprise continues. Supportive Care in Cancer 2014;22(1 suppl. 1):S99.

Mukhopadhyay S, Kwatra G, Jeyaraj P, Badyal D. Role of olanzapine in chemotherapy induced nausea and vomiting on platinum based chemotherapy patients; a randomized controlled study. Supportive Care in Cancer 2015;23(1 suppl. 1):S126-7.
Mukhopadhyay S, Kwatra G, Kingsley P, Badyal D, Mukhopadhyay B. Olanzapine, an effective and affordable adjuvant in prophylaxis for chemotherapy induced nausea and vomiting on platinum based chemotherapy patients. Clinical Therapeutics 2015;1:e108.

Mukhopadhyay S, Kwatra G, Pamela A, Badyal D. Erratum to: Role of olanzapine in chemotherapy-induced nausea and vomiting on platinum-based chemotherapy patients: a randomized controlled study. Supportive Care in Cancer 2017;25(1):155-6.

Nakagaki 2017 \{published and unpublished data\}

Nakagaki M, Barras M, Curley C, Butler J, Kennedy G. A randomized trial of olanzapine and palonosetron versus infused ondansetron for the treatment of chemotherapy induced nausea and vomiting in patients undergoing hematopoietic stem cell transplantation. Blood 2015;126(23):1910.

* Nakagaki M, Barras M, Curley C, Butler J, Kennedy G. A randomized trial of olanzapine versus palonosetron versus infused ondansetron for the treatment of breakthrough chemotherapy-induced nausea and vomiting in patients undergoing hematopoietic stem cell transplantation. Supportive Care in Cancer 2017;25(2):607-13.

\section{Navari 2010b \{published data only\}}

Navari R. Treatment of cancer related anorexia with megestrol acetate and olanzapine. Psycho-Oncology 2009;18:S47-8.

Navari R, Brenner M. Treatment of cancer related anorexia with olanzapine and megestrol acetate. Supportive Care in Cancer 2009;17(7):971.

Navari R, Brenner M. Treatment of cancer-related anorexia with olanzapine and megestrol acetate. Journal of Clinical Oncology 2008;26(15 suppl):9576.

* Navari R, Brenner M. Treatment of cancer-related anorexia with olanzapine and megestrol acetate: a randomized trial. Supportive Care in Cancer 2010; Vol. 18, issue 8:951-6.

\section{Navari 2013b \{published data only\}}

Navari R, Nagy C, Gray S. Olanzapine versus metoclopramide for the treatment of breakthrough chemotherapy-induced nausea and vomiting (CINV) in patients receiving highly emetogenic chemotherapy. Supportive Care in Cancer 2012;20:S47.

Navari R, Nagy C, Gray S. The use of olanzapine versus metoclopramide for the treatment of breakthrough chemotherapy-induced nausea and vomiting (CINV) in patients receiving highly emetogenic chemotherapy. Journal of Clinical Oncology 2012;30(15 suppl 1):-.

Navari R, Nagy C, Gray S. The use of olanzapine versus metoclopramide for the treatment of breakthrough chemotherapy-induced nausea and vomiting in patients receiving highly emetogenic chemotherapy. Supportive Care in Cancer 2013; Vol. 21, issue 6:1655-63. 


\section{Navari 2016a \{published data only\}}

Ahlborn M. Olanzapine: extension of preventive options for nausea and vomiting after highly emetogenic chemotherapy [Olanzapin: erweiterung der praventionsmoglichkeiten von ubelkeit und erbrechen nach hoch emetogener]. Chemotherapie Onkologe 2016;22(12):992-3.

Mammatas L, Konings I. Olanzapine prevents nausea and vomiting from chemotherapy [Olanzapine voorkomt misselijkheid en braken bij chemotherapie]. Nederlands Tijdschrift voor Geneeskunde 2016;160(41):43.

* Navari R, Qin R, Ruddy J, Liu H, Powell S, Bajaj M, et al. Olanzapine for the prevention of chemotherapy-induced nausea and vomiting. New England Journal of Medicine. NEJM, 2016; Vol. 375:134-42.

Navari R, Qin R, Ruddy K, Liu H, Powell S, Bajaj M, et al. Olanzapine for the prevention of chemotherapy-induced nausea and vomiting (CINV) in patients receiving highly emetogenic chemotherapy (HEC): Alliance A221301, a randomized, double-blind, placebo-controlled trial. Journal of Clinical Oncology 2015;33((29 suppl. 1)):176.

Nikbakhsh 2016 \{published data only\}

Nikbakhsh N, Sadeghi M, Ramzani E, Moudi S, Bijani A, Yousefi R, et al. Efficacy of olanzapine in symptom relief and quality of life in gastric cancer patients receiving chemotherapy. Journal of Research in Medical Sciences 2016;21:88.

\section{Shumway 2015 \{published data only\}}

Shumway N, Terrazzino S, Jones C. A randomized pilot study comparing aprepitant to olanzapine for treatment of chemotherapy-induced nausea and vomiting [abstract no. 9633]. Journal of Clinical Oncology 2009;27((15S Part I)):516.

* Shumway N, Terrazzino S, Jones C. A randomized pilot study comparing olanzapine (Zyprexa) to aprepitant (Emend) for treatment of chemotherapy induced nausea and vomiting. Journal of Pain Management 2015;8(3):233-41.

\section{Wang 2015 \{published data only\}}

Wang $\mathrm{X}$, Wang L, Wang $\mathrm{H}$, Zhang $\mathrm{H}$. Effectiveness of olanzapine combined with ondansetron in the prevention of chemotherapy-induced nausea and vomiting of non-small cell lung cancer. Cell Biochemistry and Biophysics 2015;72:471-3.

\section{Zhang 2017 \{unpublished data only\}}

Zhang D. Aprepitant, olanzapine, palonosetron and dexamethasone for the prevention of chemotherapy-induced nausea and vomiting (AOPDPCINV). clinicaltrials.gov/ct2/show/ NCT02484911 (accessed 28 September 2017).

\section{Zhao 2014 \{published data only\}}

Zhao J, Li X, Jinghua G, Li Y, Li J, Shi Y, et al. Clinical observation of olanzapine for prevention of high and moderate emetic risk chemotherapy - induced nausea and vomiting. Modern Oncology. Modern Oncology, 2014; Vol. 22, issue 8:1941-3.

\section{References to studies excluded from this review}

Babu 2016 \{published data only\}

Babu G, Saldanha S, Kuntegowdanahalli C, Jacob L, Mallekavu S, Dasappa L, et al. The efficacy, safety, and cost benefit of olanzapine versus aprepitant in highly emetogenic chemotherapy: a pilot study from South India. Chemotherapy Research and Practice 2016;-:(no pagination).

EUCTR2015-002294-38-DK \{unpublished data only\}

EUCTR2015-002294-38-DK. DANSAC-pilot: an open label pilot study to investigate the tolerability and efficacy of olanzapine in patients with advanced cancer not receiving chemotherapy or irradiation. apps.who.int/trialsearch/ Trial2.aspx?TrialID=EUCTR2015-002294-38-DK (accessed 28 September 2017).

\section{Flank 2015a \{published data only\}}

Flank J, Robinson P, Holdsworth M, Portwine C, Gibson P, Phillips R, et al. Guideline for the prevention of breakthrough and treatment of refractory chemotherapy-induced nausea and vomiting in pediatric cancer patients. Canadian Journal of Hospital Pharmacy 2015;68(1):87-8.

\section{Flank 2015b \{published data only\}}

Flank J, Thackray J, Nielson D, August A, Schechter T, Alexander $\mathrm{S}$, et al. Olanzapine for treatment and prevention of acute chemotherapy-induced vomiting (CIV) in children: a retrospective, multi-centre review. Supportive Care in Cancer 2014;22(1 Suppl. 1):S157.

* Flank J, Thackray J, Nielson D, August A, Schechter T, Alexander $\mathrm{S}$, et al. Olanzapine for treatment and prevention of acute chemotherapy-induced vomiting in children: a retrospective, multi-center review. Pediatric Blood and Cancer 2015;62(3):496-501.

Flank J, Thackray J, Nielson D, August A, Schechter T, Alexander $\mathrm{S}$, et al. Olanzapine for treatment and prevention of acute chemotherapy-induced vomiting in children: a retrospective, multi-centre review. Pediatric Blood and Cancer 2014;61:S241.

\section{Fountaine 2010 \{published data only\}}

Fountaine R, Taylor A, Mancuso J, Greenway F, Byerley L, Smith $S$, et al. Increased food intake and energy expenditure following administration of olanzapine to healthy men. Obesity (Silver Spring, Md.) 2010;18(8):1646-51.

\section{Guntsch 2012 \{published data only\}}

Guntsch F, Jahn F, Stein A, Russel J, Timo B, Wass M, et al. COMFORT trial-comparison of olanzapin and metoclopramid for treatment of breakthrough emesis: first experiences of this trial in progress. Onkologie 2012;35:76.

\section{Hashimoto 2016 \{published data only\}}

Hashimoto H, Yanai T, Nagashima K, Tsuda N, Horinouchi H, Takiguchi T, et al. A double-blind randomized phase II study of 10 versus $5 \mathrm{mg}$ olanzapine for emesis induced by highly emetogenic chemotherapy with cisplatin. Journal of Clinical Oncology 2016;34:(no pagination). 
ISRCTN58624349 \{unpublished data only\}

ISRCTN58624349. Olanzapine (ZYPREXA) versus haloperidol (Novo-Peridol) for the relief of nausea and vomiting (N\&V) in patients with advanced cancer. www.isrctn.com/ ISRCTN58624349?q=\&filters=conditionCategory:Signs\%20and $\% 20$ Symptoms,trialStatus:Stopped\&sort=relevance\&offset=23\&totalR search (accessed 28 September 2017).

Kwatra 2013 \{published data only (unpublished sought but not used)\}

Kwatra G, Badyal D K, Alice K, Mukhopadhyay P S. Olanzapine for post-chemotherapy dysgeusia and anorexia: The unidentified saviour?. Supportive Care in Cancer 2013;21:S79.

Long 2017 \{published and unpublished data\}

* Long C, Knoderer H, Mueller E. A pilot study comparing the addition of olanzapine or aprepitant in an antiemetic regimen for highly emetogenic chemotherapyPediatric Blood and Cancer. American Society of Pediatric Hematology/Oncology, ASPHO 2015;62:S104.

NCT02097823. Pilot study of olanzapine and aprepitant to prevent nausea and vomiting in children receiving chemotherapy NCT02097823. www.clinicaltrials.gov (accessed 28 September 2017).

\section{Mukhopadhyay 2012 \{published and unpublished data\}}

Mukhopadhyay S, Kwatra G, Badyal D K, Alice K. Anorexia in concurrent chemoradiation patients: Can olanzapine calm down the monster?. Indian Journal of Physiology and Pharmacology 2012;56(5 SUPPL. 1):219-20.

\section{Nakashima 2015 \{unpublished data only\}}

Nakashima K, Murakami H. A phase II study of aprepitant, palonosetron, dexamethasone and olanzapine for the prevention of cisplatin-based hemotherapy-induced nausea and vomiting for thoracic malignancy. upload.umin.ac.jp/cgi-open-bin/ctr/ctr.cgi? function=brows\&action=brows\&recptno=R000020267\&type=summar 2015.

Navari 2009a \{published data only (unpublished sought but not used)\}

Navari R, Gray S. Treatment of chemotherapy-induced breakthrough nausea and vomiting. Journal of Clinical Oncology 2009;1(27(supp)):e20536.

Navari 2010a \{published data only (unpublished sought but not used)\}

Navari R, Gray S. Treatment of chemotherapy-induced breakthrough nausea and vomiting. Psycho-oncology 2010;19(supp):S1-102.

\section{Navari 2011 \{published data only\}}

Navari R, Gray S, Kerr A. Olanzapine versus aprepitant for the prevention of chemotherapy induced nausea and vomiting: a randomized Phase III trial. Psycho-oncology 2012;21:111-2.

Navari R, Gray S, Kerr A. Olanzapine versus aprepitant for the prevention of chemotherapy-induced nausea and vomiting
(CINV): a randomized phase III trial. Journal of Clinical Oncology 2010;28((15 SUPPL. 1)):-.

* Navari R, Gray S, Kerr A. Olanzapine versus aprepitant for the prevention of chemotherapy-induced nausea and vomiting: a randomized phase III trial. The Journal of Supportive Oncology 2011; Vol.9, issue 5.188-95.

Navari R, Gray S, Kerr, A. Olanzapine versus aprepitant for the prevention of chemotherapy induced nausea and vomiting (CINV): a randomized trial. Supportive Care in Cancer 2010;18:S81-2.

\section{Navari 2016b \{published data only\}}

Navari R, Nagy C. Olanzapine versus fosaprepitant for the prevention of nausea and vomiting in patients receiving concurrent chemoradiation treatment. Journal of Clinical Oncology 2015;33(15 suppl. 1):-.

* Navari R, Nagy C, Le-Rademacher J, Loprinzi C. Olanzapine versus fosaprepitant for the prevention of concurrent chemotherapy radiotherapy-induced nausea and vomiting. The Journal of Community and Supportive Oncology 2016;14(4):141-7.

\section{NCT00124930 \{unpublished data only\}}

NCT00124930. Study comparing olanzapine with haloperidol for the relief of nausea and vomiting in patients with advanced cancer. clinicaltrials.gov/ct2/show/NCT00124930 (first received 29 July 2005).

\section{NCT01148264 \{unpublished data only\}}

NCT01148264. Comparison of olanzapine and metoclopramide for treatment of breakthrough emesis (COMFORT). www.clinicaltrials.gov (accessed 28 September 2017).

Slimano 2016 \{published and unpublished data\}

Slimano F, Netzer F, Borget I, Lemare F, Besse B. Is the addition of olanzapine effective in restoration of CINV prevention in nony sespormages= to standard antiemetic therapy? A retrospective controlled study. International Journal of Clinical Pharmacy 2016;38(6):491.

Yanai 2015 \{unpublished data only\}

Yanai T, Yamamoto N. A double-blind randomized phase II study of olanzapine $10 \mathrm{mg}$ versus $5 \mathrm{mg}$ for highly emetogenic chemotherapy-induced nausea and vomiting. rctportal.niph.go.jp/en/detail?trial_id=UMIN000014214 2015.

\section{References to studies awaiting assessment}

Chasick 2012 \{published data only\}

Chasick A, DeFreitas E, Edwards M. Olanzapine for the prevention of chemotherapy-induced nausea and vomiting in patients receiving highly or moderately emetogenic antineoplastic chemotherapy. Journal of the American Pharmacists Association 2012;52(5):681. 
CTRCC-14004093 \{published data only (unpublished sought but not used)\}

ChiCTR-TTRCC-14004093. Olanzapine versus aprepitant for the prevention of high dose cisplatin-induced nausea and vomiting: a randomized phase III trial. apps.who.int/trialsearch/ Trial2.aspx?TrialID=ChiCTR-TTRCC-14004093 (accessed 28 September 2017).

\section{Jeon 2017 \{published data only\}}

Jeon S, Han H, Bae W, Park M, Lee S, Go S, et al. A randomized, double-blind, placebo-controlled study of the safety and efficacy of olanzapine for the prevention of chemotherapyinduced nausea and vomiting in patients receiving moderately emetogenic chemotherapy. Journal of Clinical Oncology 2017;35(15 Supplement 1):(no pagination).

Mao 2011 \{published data only (unpublished sought but not used)\} Mao W-K, Peng L. Clinical observation of olanzepine combined with granisterom and hexadecadrol prevent nausea vomit induced by chemoradiotherapy. Chinese Journal of Medicinal Guide 2011;13(3):452-4.

\section{Meng 2016 \{published data only (unpublished sought but not used)\}}

Meng Q, Chen G, Guo P. Olanzapine combined with normal antiemetic drugs in patients on solid tumor chemotherapy: antiemetic effect and impact on quality of life. World Chinese Journal of Digestology 2016;24(7):1117-23.

Mukesh 2017 \{published data only (unpublished sought but not used)\}

Mukesh S, Sathya M, Akshay J. Olanzapine versus aprepitant in the prevention of chemotherapy induced nausea and vomiting (CINV) in breast cancer patients. Journal of Clinical Oncology 2017;35(15 Supplement 1):-.

\section{Nguyen 2017 \{published data only (unpublished sought but not} used)\}

Nguyen N, Pham V, Nguyen T, Tran T. Olanzapine and omeprazole combination is simple, safe and effective for delayed nausea and vomiting control in adjuvant chemotherapy for early stage breast cancer. Supportive Care in Cancer 2017;25(2 Supplement 1):S72.

Wang 2012 \{published data only\}

Wang, Wang. Effectiveness of olanzapine in prevention of chemotherapy induced nausea and vomiting. Clin J Clin (full journal title not available) 2012;6:7406-7.

\section{References to ongoing studies}

\section{Abe 2017 \{unpublished data only\}}

Abe M, Yanai T, Nakashima K, Hirashima Y, Murakami H, Hamauchi S, et al. A randomized, double-blind, placebocontrolled phase iii study evaluating olanzapine $5 \mathrm{mg}$ combined with standard antiemetic therapy for the prevention of CINV in patients receiving cisplatin-based chemotherapy. Supportive Care in Cancer. Conference: 2017 International MASCC/ISOO Symposium 2017;25((2 Supplement 1)):S632017.

\section{ChicTR-TTRCC-14004093 \{unpublished data only\}}

ChiCTR-TTRCC-14004093. Olanzapine versus aprepitant for the prevention of high-dose cisplatin-induced nausea and vomiting: a randomised phase III trial. apps.who.int/trialsearch/ Trial2.aspx?TrialID=ChiCTR-TTRCC-14004093 (accessed 28 September 2017) (first received 08 January 2014).

Hashimoto 2017 \{published data only (unpublished sought but not used)\}

Hashimoto $\mathrm{H}$, Abe M. A randomized, double-blind, placebocontrolled phase III trial evaluating olanzapine $5 \mathrm{mg}$ combined with standard antiemetic therapy for the prevention of chemotherapy-induced nausea and vomiting in patients receiving cisplatin-based highly emetogenic chemotherapy: J-SUPPORT 1604. rctportal.niph.go.jp/en/detail? trial_id=UMIN000024676 2017;-:-

Hashimoto H, Iwasa S, Abe M, Yanai T, Zenda S, Yamaguchi T, et al. 1607TiP - J-FORCE study: a randomized, double-blind, placebo-controlled phase III study evaluating olanzapine $(5 \mathrm{mg})$ combined with standard antiemetic therapy. Annals of Oncology 2017;28(suppl_5):v543-67.

\section{JPRN-UMIN000010317 \{unpublished data only\}}

JPRN-UMIN000010317. Efficacy of olanzapine for relief of nausea with incomplete bowel obstruction in advanced cancer patient: pragmatic randomized controlled trial. apps.who.int/ trialsearch/Trial2.aspx?TrialID=JPRN-UMIN000010317 (first received 26 March 2013).

Mukhopadhyay 2017a \{published data only (unpublished sought but not used)\}

Mukhopadhyay S, Dutta P, Bhattacharya B, Banerjee S, Biswas S, Mukhopadhyay-Samanta B. Low dose vs. standard dose adjuvant olanzapine in chemotherapy induced nausea and vomiting: a prospective, randomized, double blinded, controlled study. Clinical Therapeutics 2017;39(8 Supplement 1):e17.

Mukhopadhyay S, Dutta P, Bhattacharya B, Banerjee S, Biswas S, Samanta B. Comparison of efficacy, safety and sedation with two doses of add on olanzapine in chemotherapy induced nausea and vomiting: a randomized controlled pilot study. Supportive Care in Cancer 2016;24(1 Supplement 1):S248-9.

Mukhopadhyay S, Dutta P, Bhattacharya B, Das D, Biswas S. Low dose olanzapine in chemotherapy induced nausea and vomiting: a balance game between sedation and nausea?. Supportive Care in Cancer: International MASCC/ISOO Symposium 2017;25(2 Supplement 1):S69.

\section{Nagashima et al 2015 \{published data only\}}

Nagashima K, Iwasa S, Yanai T, Hashimoto H, Suzuki K, Ohyanagi $F$, et al. A double-blind randomized phase II study of olanzapine $10 \mathrm{mg}$ versus $5 \mathrm{mg}$ for emesis induced by highly emetogenic chemotherapy. Japanese Journal of Clinical Oncology 2015;45(2):229-31.

\section{NCT02290470 \{unpublished data only\}}

NCT02290470. Olanzapine against delayed nausea and vomiting in women receiving carboplatin plus paclitaxel. 
clinicaltrials.gov/ct2/show/NCT02290470 (first received 14 November 2014).

\section{NCT02400866 \{unpublished data only\}}

NCT02400866. A randomized study of olanzapine for the prevention of CINV in patients receiving moderately emetogenic chemotherapy. clinicaltrials.gov/ct2/show/NCT02400866 (first received 27 March 2015).

\section{NCT02635984 \{unpublished data only\}}

NCT02635984. Study of FOND versus FOND+O for the prevention of CIV in hematology patients receiving highly emetogenic chemotherapy regimens [Randomized, placebo controlled study of FOND (fosaprepitant, ondansetron, dexamethasone) versus FOND+O (FOND plus olanzapine) for the prevention of chemotherapy induced nausea and vomiting in hematology patients receiving highly emetogenic chemotherapy regimens]. clinicaltrials.gov/ct2/show/NCT02635984 (first received 21 December 2015).

\section{NCT02939287 \{unpublished data only\}}

NCT02939287. Aprepitant versus olanzapine with high dose melphalan [Aprepitant versus olanzapine for prevention of acute and delayed nausea and vomiting associated with high dose melphalan and BEAM in autologous stem dell transplant patients]. clinicaltrials.gov/ct2/show/NCT02939287 (first received 20 October 2016).

\section{NCT02970643 \{unpublished data only\}}

NCT02970643. Proof-of-concept trial of palonosetron and olanzapine without dexamethasone for the prevention of CIN [Proof-of-concept trial of palonosetron and olanzapine without dexamethasone for the prevention of chemotherapy-induced nausea and vomiting]. clinicaltrials.gov/ct2/show/NCT02970643 (first received 22 November 2016).

\section{NCT03079219 \{unpublished data only\}}

NCT03079219. Olanzapine for the prevention of chemotherapyinduced nausea and vomiting in Chinese breast cancer patients [A randomized study to determine the efficacy and tolerability of olanzapine for the prevention of chemotherapy-induced nausea and vomiting in Chinese breast cancer patients]. clinicaltrials.gov/ct2/show/NCT03079219 (first received 14 March 2017).

\section{NCT03137121 \{unpublished data only\}}

NCT03137121. Olanzapine for the treatment of chronic nausea and/or vomiting in advanced cancer patients [Olanzapine for the treatment of chronic nausea and/or vomiting, unrelated to chemotherapy or radiation, in advanced cancer patient - a pilot, dose-finding trial]. clinicaltrials.gov/ct2/show/NCT03137121 (first received 2 May 2017).

\section{Additional references}

\section{Anonymous 2017}

Anonymous. Zyprexa $2.5 \mathrm{mg}, 5 \mathrm{mg}, 7.5 \mathrm{mg}, 10 \mathrm{mg}, 15 \mathrm{mg}$, and $20 \mathrm{mg}$ coated tablets. Zyprexa Velotab $5 \mathrm{mg}, 10 \mathrm{mg}, 15 \mathrm{mg}$, and $20 \mathrm{mg}$ orodispersible tablets. www.medicines.org.uk/emc/ medicine/614 (accessed 29 September 2017).

\section{BNF}

British National Formulary. www.evidence.nhs.uk/formulary/ bnf/current/4-central-nervous-system/42-drugs-used-inpsychoses-and-related-disorders/421-antipsychotic-drugs/ second-generation-antipsychotic-drugs/olanzapine (accessed 28 September 2017).

\section{Burke 2011}

Burke T, Wisniewski T, Ernst F. Resource utilization and costs associated with chemotherapy-induced nausea and vomiting (CINV) following highly or moderately emetogenic chemotherapy administered in the US outpatient hospital setting. Supportive Care in Cancer 2011;19(1):131-40.

\section{Chanthawong 2017}

Chanthawong S, Lim Y H, Subongkot S, Chan A, Andalusia R, Bustamam R S, et al. Cost-effectiveness analysis of olanzapinecontaining antiemetic therapy for managing highly emetogenic chemotherapy in south east Asia: a multinational study. Supportive Care in Cancer 2017;25(2 Supplement 1):S64-5.

\section{Chelkeba 2017}

Chelkeba L, Gidey K, Mamo A, Yohannes B, Matso T, Melaku T. Olanzapine for chemotherapy-induced nausea and vomiting: systematic review and meta-analysis. Pharmacy Practice 2017;15(1):877.

\section{Chiu 2016a}

Chiu L, Chow R, Popovic M, Navari R, Shumway N, Chiu N, et al. Efficacy of olanzapine for the prophylaxis and rescue of chemotherapy-induced nausea and vomiting (CINV): a systematic review and meta-analysis. Supportive Care in Cancer 2016;24(5):2381-92.

\section{Chow 2016}

Chow R, Chiu L, Navari R, Passik S, Chiu N, Popovic M, et al. Efficacy and safety of olanzapine for the prophylaxis of chemotherapy-induced nausea and vomiting (CINV) as reported in phase I and II studies: a systematic review. Supportive Care in Cancer 2016;24(2):1001-8.

\section{Cohen 2007}

Cohen L, de Moor C, Eisenberg P, Ming E, Hu H. Chemotherapyinduced nausea and vomiting: incidence and impact on patient quality of life at community oncology settings. Supportive Care in Cancer 2007;15(5):497-503.

\section{Covidence [Computer program]}

Veritas Health Innovation. Covidence. Version 30 September 2017. Melboune, Australia: Veritas Health Innovation, 2017.

\section{Cox 2015}

Cox L, Darvill E, Dorman S. Levomepromazine for nausea and vomiting in palliative care. Cochrane Database of Systematic Reviews 2015, Issue 11. [DOI: 10.1002/14651858.CD009420.pub3]

\section{Deeks 2017}

Deeks J J, Higgins J P, Altman D G (editors) on behalf of the Cochrane Statistical Methods Group. Chapter 9: Analysing data and undertaking meta-analyses. In: Higgins J P T, 
Churchill R, Chandler J, Cumpston M S (editors), Cochrane Handbook for Systematic Reviews of Interventions version 5.2.0 (updated June 2017), Cochrane, 2017. Available from www.training.cochrane.org/handbook.

\section{Egger 1997}

Egger M, Davey Smith G, Schneider M, Minder C. Bias in meta-analysis detected by a simple, graphical test. $B M J$ 1997;315(7109):629-34.

\section{FDA}

US Food, Drug Administration (FDA). ZYPREXA ${ }^{\circledR}$ olanzapine tablets ZYPREXA ${ }^{\circledast}$ ZYDIS $^{\circledast}$ olanzapine orally disintegrating tablets ZYPREXA ${ }^{\circledR}$ intramuscular olanzapine for Injection. www.fda.gov/ ohrms/dockets/ac/08/briefing/2008-4399b1-05\%20(Zyprexa \%20(olanzapine)\%20Labeling).pdf accessed 23 October 2017.

\section{Glare 2011}

Glare P, Miller J, Nikolova T, Tickoo R. Treating nausea and vomiting in palliative care: a review. Clinical Interventions in Aging 2011;6:243-59.

\section{GRADEpro GDT 2015 [Computer program]}

MCMaster University (developed by Evidence Prime). GRADEpro GDT. Hamilton (ON): McMaster University (developed by Evidence Prime), 2015.

\section{GraphPad [Computer program]}

GraphPad Software Inc. GraphPad. Version accessed 26 October 2017. GraphPad Software Inc, 2017.

\section{Guyatt 2011}

Guyatt G H, Oxman A D, Schunemann H J, Tugwell P, Knottnerus A. GRADE guidelines: a new series of articles in the Journal of Clinical Epidemiology. Journal of Clinical Epidemiology 2011;64:380-2.

\section{Higgins 2003}

Higgins J P, Thompson S G, Deeks J J, Altman D G. Measuring inconsistency in meta-analyses. BMJ 2003;327:557-60.

\section{Higgins 2017}

Higgins J P, Altman D G, Sterne J A C (editors). Chapter 8: Assessing risk of bias in included studies. In: Higgins J P T, Churchill R, Chandler J, Cumpston M S (editors), Cochrane Handbook for Systematic Reviews of Interventions version 5.2.0 (updated June 2017), Cochrane, 2017. Available from www.training.cochrane.org/handbook.

\section{Hjermstad 2013}

Hjermstad M, Kolflaath J, Løkken A, Hanssen S, Normann A, Aass N. Are emergency admissions in palliative cancer care always necessary? Results from a descriptive study. BMJ Open 2013;3(5):e002515.

\section{Hocking 2014}

Hocking C, Kichenadasse G. Olanzapine for chemotherapyinduced nausea and vomiting: a systematic review. Supportive Care in Cancer 2014;22(4):1143-51.

\section{Jackson 2003}

Jackson W, Tavernier L. Olanzapine for intractable nausea in palliative care patients. Journal of Palliative Medicine 2003;6(2):251-5.

\section{Kishi 2015}

Kishi T, Hirota T, Matsunaga S, Iwata N. Antipsychotic medications for the treatment of delirium: a systematic review and meta-analysis of randomised controlled trials. Journal of Neurology, Neurosurgery, and Psychiatry 2016;87(7):767-74.

\section{Kris 2011}

Kris M. Impact of CINV. Clinical Advances in Hematology and Oncology 2011;9(1):9-13.

\section{Kumar 2017}

Kumar R, Singh N, Thekkekara R, Singh S, Harrington S, Shah M. Efficacy of olanzapine for prevention of chemotherapyinduced nausea and vomiting: A systematic review and metaanalysis. Journal of Clinical Oncology. Conference 2017;35(15 Supplement 1):-.

\section{Martel 2016}

Martel M, Klein L, Rivard R, Colev J. A large retrospective cohort of patients receiving intravenous olanzapine in the emergency department. Society for Academic Emergency Medicine 2016;23:29-33.

\section{Medicines.org}

EMC. Nytol One-A-Night. www.medicines.org.uk/emc/ medicine/19778 (accessed 1 November 2017).

\section{Medicines.org a}

EMC. Zofran Tablets $4 \mathrm{mg}$ and $8 \mathrm{mg}$. www.medicines.org.uk/ emc/medicine/17650/spc (accessed 1 November 2017).

\section{Moher 2009}

Moher D, Liberati A, Tetzlaff J, Altman D. Preferred reporting items for systematic reviews and meta-analyses: the PRISMA statement. PLoS Medicine 2009;6(7):e1000097.

\section{Murray-Brown 2015}

Murray-Brown F, Dorman S. Haloperidol for the treatment of nausea and vomiting in palliative care patients. Cochrane Database of Systematic Reviews 2015, Issue 11. [DOI: 10.1002/14651858.CD006271.pub3]

\section{Navari 2013a}

Navari R. Management of chemotherapy-induced nausea and vomiting: focus on newer agents and new uses for older agents. Drugs 2013;73(3):249-62.

\section{Pirl 2000}

Pirl W F, Roth A J. Remission of chemotherapy-induced emesis with concurrent olanzapine treatment: a case report. Psychooncology 2000;9(1):84-7.

Prommer 2012

Prommer E. Olanzapine: palliative medicine update. American Journal of Hospice \& Palliative Medicine 2012;30(1):75-82. 


\section{Review Manager 2014 [Computer program]}

Nordic Cochrane Centre, The Cochrane Collaboration. Review Manager 5 (RevMan 5). Version 5.3. Copenhagen: Nordic Cochrane Centre, The Cochrane Collaboration, 2014.

\section{Schünemann 2011}

Schünemann H J, Oxman A D, Higgins J P, Vist G E, Glasziou P, Guyatt G H. Chapter 11: Presenting results and 'Summary of findings' tables. In: Higgins J P T, Green S (editors), Cochrane Handbook for Systematic Reviews of Interventions Version 5.1.0 (updated March 2011). The Cochrane Collaboration, 2011. Available from www.handbook.cochrane.org.

\section{Schünemann 2017}

Schünemann H J, Oxman A D, Higgins J P, Vist G E, Glasziou P, Akl E, et al. on behalf of the Cochrane GRADEing Methods Group and the Cochrane Statistical Methods Group. Chapter 11: Completing 'Summary of findings' tables and grading the confidence in or quality of the evidence. In: Higgins J P T, Churchill R, Chandler J, Cumpston M S (editors), Cochrane Handbook for Systematic Reviews of Interventions version 5.2.0 (updated June 2017). Cochrane, 2017. Available from www.training.cochrane.org/handbook.

\section{Solano 2006}

Solano J, Gomes B, Higginson I. A comparison of symptom prevalence in far advanced cancer, AIDS, heart disease, chronic obstructive pulmonary disease and renal disease. Journal of Pain and Symptom Management 2006;31(1):58-69.

\section{Sommariva 2016}

Sommariva S, Pongiglione B, Tarricone R. Impact of chemotherapy-induced nausea and vomiting on health-related quality of life and resource utilization: a systematic review. Critical Reviews in Oncology/Hematology 2016;99:13-36.

\section{CHARACTERISTICS OF STUDIES}

\section{Characteristics of included studies [ordered by study ID]}

\section{Teunissen 2007}

Teunissen S, Wesker W, Kruitwagen C, De Haes H, Voest E, De Graeff A. Symptom prevalence in patients with incurable cancer: a systematic review. Journal of Pain and Symptom Management 2007;31(1):94-104.

\section{van der Vorst 2015}

van der Vorst M, Neefjes E, Konings I, Verheul H. Prophylactic treatment for delayed chemotherapy-induced nausea and vomiting after non-AC based moderately emetogenic chemotherapy: a systematic review of randomized controlled trials. Supportive Care in Cancer 2015;23(8):2499-506.

\section{Wang 2014}

Wang S, Yang Z, Zhang L. Olanzapine for preventing nausea and vomiting induced by moderately and highly emetogenic chemotherapy. Asian Pacific Journal of Cancer Prevention 2014;15(22):9587-92.

\section{Yang 2017}

YangT, Liu Q, Lu M, Ma L, Zhou Y, Cui Y. Efficacy of olanzapine for the prophylaxis of chemotherapy-induced nausea and vomiting: a meta-analysis. British Journal of Clinical Pharmacology 2017;83(7):1369-79.

\section{Yoodee 2017}

Yoodee J, Permsuwan U, Nimworapan M. Efficacy and safety of olanzapine for the prevention of chemotherapy-induced nausea and vomiting: A systematic review and meta-analysis. Critical Reviews in Oncology/Hematology 2017;112:-.

* Indicates the major publication for the study

Clemons 2016

$\begin{array}{ll}\text { Methods } & \text { Trial method: RCT } \\ & \text { Randomised: yes } \\ & \text { Blinding: } \\ \text { Arms: } 2 & \\ & \text { MEC or HEC: HEC + MEC } \\ & \text { Multicentre/single-centre: } 2 \\ & \text { Dates: April 2012-September } 2014 \\ & \text { N }=324 \\ & \text { Age: } 26-76 \text { years } \\ \text { Gender: Female } \\ \text { Inclusion criteria: newly diagnosed stage I-III breast cancer and scheduled to receive chemotherapy } \\ \text { with a cyclophosphamide- and anthracycline-containing regimen } \\ \text { Exclusion criteria: } \\ \text { Participant (baseline) characteristics: main diagnosis: breast cancer } \\ \text { Setting and location: hospitals, Canada } \\ \text { Number of people screened: } 328\end{array}$


Number of participants randomised - all: 324

Number randomised to each group: 154 risk-model guided, 170 physicians' choice

Number receiving treatment as allocated: 324

Number not receiving treatment as allocated: 0

Number dropped out: 5 from physician's choice, 7 from risk-model guided

(no follow-up data for any outcome available)

Number excluded from analysis (for all outcomes): 0

Interventions $\quad \mathrm{N}=\mathbf{1 5 4}$ in intervention group $\mathrm{A}, \mathrm{B}, \mathrm{C}$

Intervention group $\mathrm{A}$ : ( $\mathrm{N}=$ ?) Low risk: day 1 , IV dexamethasone, $10 \mathrm{mg}$, and oral ondansetron, $8 \mathrm{mg}$, before chemotherapy; oral dexamethasone, $4 \mathrm{mg}$, and oral ondansetron, $8 \mathrm{mg}, 8$ hours later; day 2 and 3 , oral dexamethasone, $4 \mathrm{mg}$ twice daily, and oral ondansetron, $8 \mathrm{mg}$ twice daily

Intervention group $\mathrm{B}$ : ( $\mathrm{N}=$ ?) high risk: day 1 , IV dexamethasone, $12 \mathrm{mg}$, oral ondansetron, $8 \mathrm{mg}$, and oral aprepitant, $125 \mathrm{mg}$, before chemotherapy; and oral ondansetron, $8 \mathrm{mg}$, 8 hours later; day 2 and 3 , oral aprepitant, $80 \mathrm{mg}$ once daily, alone

Intervention group C: ( $\mathrm{N}=$ ?) ongoing high risk: additional dexamethasone and $2.5 \mathrm{mg} / \mathrm{d}$ olanzapine as well as day 1, IV dexamethasone, $12 \mathrm{mg}$, oral ondansetron, $8 \mathrm{mg}$, and oral aprepitant, $125 \mathrm{mg}$, before chemotherapy; and oral ondansetron, $8 \mathrm{mg}, 8$ hour later; day 2 and 3, oral aprepitant, $80 \mathrm{mg}$ once daily, alone

Control Group C: ( $N=170)$ physician's choice - some participants also received olanzapine

Use of additional treatments if any (co-interventions, additional analgesia): prochlorperazine and methotrimeprazine for the control of protracted nausea and vomiting, IV fluids, metoclopramide, and non-prescribed antiemetic drugs

Outcomes of interest in the review:
Primary outcomes
- complete control of nausea and vomiting in the acute and delayed periods after chemotherapy
Secondary outcomes
- day 5 after each chemotherapy cycle consisted of overall patient satisfaction
- QOL was assessed before the start of chemotherapy, at 24 hour, and at day 5 using the FLIE index; both
nausea and vomiting control using a 4-point Likert scale

Other outcomes reported by the study: none

\begin{tabular}{ll}
\hline Funding & Canadian Breast Cancer Research Foundation-Ontario Chapter \\
\hline Declarations of Interest & None declared \\
\hline Notes & Meets inclusion criteria but no data to use
\end{tabular}

\section{Risk of bias}

\begin{tabular}{lll}
\hline Bias & Authors' judgement & Support for judgement \\
\hline $\begin{array}{l}\text { Random sequence genera- } \\
\text { tion (selection bias) }\end{array}$ & Unclear risk & $\begin{array}{l}\text { Quote: "Eligible and consented patients were randomized (one to one) to } \\
\text { the RMG [risk-model group) group or to the PC [physician's choice) control } \\
\text { group..." } \\
\text { Comment: method of randomisation was not described }\end{array}$ \\
\hline $\begin{array}{l}\text { Allocation concealment } \\
\text { (selection bias) }\end{array}$ & Unclear risk & Comment: method of allocation was not described \\
\hline $\begin{array}{l}\text { Blinding of participants } \\
\text { and personnel (perfor- } \\
\text { mance bias) }\end{array}$ & High risk & $\begin{array}{l}\text { Quote: "For patients randomized to the PC control group, the treating oncolo- } \\
\text { gist could choose any combination, dose, and duration of antiemetic therapy } \\
\text { he or she wished for each cycle of chemotherapy." }\end{array}$ \\
\hline
\end{tabular}


Clemons 2016 (Continued)

All outcomes
Comment: participants and personnel were therefore aware if the participant was in the intervention or control arm

\section{Blinding of outcome as- High risk} sessment (detection bias)

All outcomes
Quote: "Patients were provided with a diary to record the number of episodes as well as the intensity and duration of nausea and vomiting during the first 24 hours and during days 2 to 5 following chemotherapy. This was supplemented with a telephone call by the study coordinator on $\mathrm{d}$ one and 5 after chemotherapy."

Comment: low-risk methods but in the context of unblinded participants and personnel this represents a high risk of bias

\begin{tabular}{|c|c|c|}
\hline $\begin{array}{l}\text { Incomplete outcome data } \\
\text { (attrition bias) }\end{array}$ & Low risk & $\begin{array}{l}\text { Comment: } 12 \text { participants had no evaluable data out of } 324 \text { randomised par- } \\
\text { ticipants }(3.7 \%) \text {. See CONSORT diagram (Figure } 1 \text { ) }\end{array}$ \\
\hline
\end{tabular}

Comment: 12 participants had no evaluable data out of 324 randomised par-

\begin{tabular}{lll}
\hline $\begin{array}{l}\text { Selective reporting (re- } \\
\text { porting bias) }\end{array}$ & High risk & $\begin{array}{l}\text { Comment: reported primary and secondary outcomes were different from } \\
\text { those stipulated in the protocol (clinicaltrials.gov NCT01913990) }\end{array}$ \\
\hline $\begin{array}{l}\text { Other bias (validation of } \\
\text { instruments) }\end{array}$ & Low risk & Validated FLIE scale used \\
\hline Other bias & Unclear risk & Unclear risk of bias based on number of participants per arm (154 and 170) \\
\hline
\end{tabular}

\title{
Liu/Tan 2015
}

\section{Liu 2015}

Trial method: RCT

Randomised: yes - random digits table

Blinding: unblinded

Arms: 2

\section{MEC or HEC: MEC/HEC}

Multicentre/single-centre: single

Dates: January 2008-August 2008

\section{Tan 2009}

Trial method: RCT randomised: yes

Blinding: unblinded

Arms: 2

MEC or HEC: MEC/HEC

Multicentre/single-centre: single

Dates: January 2008-August 2008

Participants Liu 2005

\author{
$N=229$ \\ Age: $18-74$ years \\ Gender: Male + Female
}

Inclusion criteria: pathological diagnosis of malignant cancer, or previously received chemotherapy, adequate bone marrow, renal and liver function, normal cardiac function, ECOG $\leq 2$ 
Liu/Tan 2015 (Continued)

Exclusion criteria: nausea in previous 24 hour, chemotherapy in previous 24 hour, severe cognitive impairment, CNS disease (including uncontrolled brain metastases, seizures), psychotic medications, concurrent abdominal radiotherapy, hypersensitivity to olanzapine, uncontrolled diabetes mellitus, concurrent medical disease

Participant (baseline) characteristics: main diagnosis lung, stomach, breast, ovarian, lymphoma, oesophageal, colorectal, oropharyngeal, teratoma, gingival, thymus, cervical, laryngeal, malignant melanoma, glioblastoma

Setting and location: hospital, China

Number of people screened: 229

Number of participants randomised - all: 229

Number randomised to each group: 121 olanzapine, 108 control

Number receiving treatment as allocated: 229

Number not receiving treatment as allocated: 229

Number dropped out: 0

Number excluded from analysis (for all outcomes): 0

Number completed: 229

\section{Tan 2009}

$N=229$

Age: $18-74$ years

Gender: Male + Female

Inclusion criteria: pathological diagnosis of malignant disease or previous chemotherapy, receiving MEC or HEC

Exclusion criteria: no history of CNS disease (e.g. uncontrolled brain metastases, seizure disorder) Participant (baseline) characteristics: main diagnosis lung, stomach, breast, ovarian, lymphoma, oesophageal, colorectal, oropharyngeal, teratoma, gingival, thymus, cervical, laryngeal, malignant melanoma, glioblastoma

Setting and location: hospital, China

Number of people screened: 229

Number of participants randomised - all: 229

Number randomised to each group: 121 olanzapine, 108 control

Number receiving treatment as allocated: 229

Number not receiving treatment as allocated: 229

Number dropped out: 0

Number excluded from analysis (for all outcomes): 0

Number completed: 229

Number completed: 121 olanzapine arm, 108 control arm

\section{Liu 2015}

Intervention group $\mathbf{A}(\mathrm{N}=121)$

- day 1 , olanzapine $10 \mathrm{mg}$ oral, azasetron $10 \mathrm{mg}$ IV, dexamethasone $10 \mathrm{mg} \mathrm{IV}$

- day 2-5, olanzapine $10 \mathrm{mg}$ oral daily

Control group $\mathbf{B}(\mathrm{N}=108)$

- day 1 , azasetron $10 \mathrm{mg}$ IV, dexamethasone $10 \mathrm{mg}$ IV

- day 2-5, dexamethason $10 \mathrm{mg}$ IV daily

Use of additional treatments if any: permitted to use other antiemetics - not stated what, oral estazolam for insomnia

Tan 2009

Intervention group A $(\mathrm{N}=121)$

- day 1 , olanzapine $10 \mathrm{mg}$ oral, azasetron $10 \mathrm{mg}$ IV, dexamethasone $10 \mathrm{mg}$ IV 
Liu/Tan 2015 (Continued)

- day 2-5, olanzapine $10 \mathrm{mg}$ oral daily

Control group $\mathbf{B}(N=108)$

- day 1 , azasetron $10 \mathrm{mg}$ IV, dexamethasone $10 \mathrm{mg}$ IV

- day 2-5, dexamethasone $10 \mathrm{mg}$ IV daily

Use of additional treatments if any (co-interventions, additional analgesia): oral estazolam for insomnia

Liu 2015
Outcomes of interest in the review:
Primary outcomes
- Complete response (no nausea, no vomiting, no rescue medications)
Secondary outcomes
- QOL (EORTC QLQ-C30)
- Side effects/AEs/toxicities
Tan 2009
Outcomes of interest in the review:
Primary outcomes:
- Complete response (no nausea, no vomiting, no rescue medications)
Secondary outcomes:
- QOL (EORTC QLQ-C30)
- Side effects/AEs/toxicities

\begin{tabular}{ll}
\hline Funding & No information \\
\hline Declarations of Interest & No information (Tan 2009: none declared) \\
\hline Notes & \\
\hline
\end{tabular}

\section{Risk of bias}

\begin{tabular}{|c|c|c|}
\hline Bias & Authors' judgement & Support for judgement \\
\hline $\begin{array}{l}\text { Random sequence genera- } \\
\text { tion (selection bias) }\end{array}$ & Low risk & $\begin{array}{l}\text { Quote: "All eligible participants were randomised... using a random digits ta- } \\
\text { ble." Liu } 2015\end{array}$ \\
\hline $\begin{array}{l}\text { Allocation concealment } \\
\text { (selection bias) }\end{array}$ & Unclear risk & Comment: method of allocation was not described \\
\hline $\begin{array}{l}\text { Blinding of participants } \\
\text { and personnel (perfor- } \\
\text { mance bias) } \\
\text { All outcomes }\end{array}$ & High risk & $\begin{array}{l}\text { Quote: "On the day of chemotherapy, day } 1 \text {, the test group patients received } \\
\text { the antiemetic regimen consist of olanzapine } 10 \mathrm{mg} \text { p.o., azasetron } 10 \mathrm{mg} \text {, i.v. } \\
\text { and dexamethasone } 10 \mathrm{mg} \text { i.v., the control group patients received a standard } \\
\text { pre-treatment antiemetic regimen consist of azasetron } 10 \mathrm{mg} \text {, i.v. and dexam- } \\
\text { ethasone } 10 \mathrm{mg} \text {, i.v. Day 2-5, the test group patients received olanzapine } 10 \mathrm{mg} \\
\text { p.o., the control group patients received dexamethasone } 10 \mathrm{mg} \text {, i.v." Tan } 2009 \\
\text { Comment: intervention arm got a tablet and IV infusion on d 1, then a tablet } \\
\text { day 2-5. Comparison group got only IV infusions on day } 1 \text { then daily IV infu- } \\
\text { sions day 2-5. Therefore not possible to blind either participants or personnel }\end{array}$ \\
\hline
\end{tabular}


Liu/Tan 2015 (Continued)

Blinding of outcome assessment (detection bias)

All outcomes
High risk

Quote: "At days 1-5 post chemotherapy patients used the observation table of CINV to record the response of the patients (mainly recorded the degree of CINV, as well as whether to take the remedial treatment to relieve nausea and vomiting), at same time patients were instructed to fill the EORTC QLQ-C30 QoL observation table on day 0 and day 6." Tan 2009 Comment: low-risk method but in the context of unblinded participants selfreporting represents a high risk of bias
Incomplete outcome data Low risk (attrition bias)

All outcomes

Quote: "229 patients were randomly enrolled onto the study. All patients were evaluable for efficacy and toxicity." Tan 2009

Comment: No CONSORT diagram available. It is stated that data for all 229 participants was reported. However, both the intervention and control group data are presented as a percentage rather than absolute numbers with each group subdivided into those receiving HEC or MEC. It is therefore not possible to extrapolate the number of participants in the HEC and MEC subgroup of each arm in Tan 2009. However, in Liu 2015 olanzapine and control groups are not subdivided into HEC vs MEC therefore it is possible to account for all participants

\begin{tabular}{lll}
\hline $\begin{array}{l}\text { Selective reporting (re- } \\
\text { porting bias) }\end{array}$ & Unclear risk & $\begin{array}{l}\text { Comment: no protocol available on clincaltrials.gov or Chinese registry of clin- } \\
\text { ical trials (www.chictr.org.cn) }\end{array}$ \\
\hline $\begin{array}{l}\text { Other bias (validation of } \\
\text { instruments) }\end{array}$ & Low risk & $\begin{array}{l}\text { Comment: AEs graded using CTCAE V 3.0, an unvalidated scale but no data } \\
\text { presented. Efficacy presented as dichotomous data and QOL presented on a } \\
\text { validated scale }\end{array}$ \\
\hline Other bias & Unclear risk & $\begin{array}{l}\text { Comment: unclear risk of bias based on number of participants per arm (121 } \\
\text { and 108) }\end{array}$ \\
\hline
\end{tabular}

\section{Lu 2013}

Trial method: RCT randomised: yes
Blinding: unclear
Arms: 2
MEC or HEC: MEC and HEC
Multicentre/single-centre: single
Dates: not stated
N $=60$ (30 per arm)
Age: $31-72$ years
Gender: Male + Female
Inclusion criteria: patients with solid tumour chemotherapy. Diagnosis was based on pathology
or sputum cytology. The relevant inspection found no contraindications to chemotherapy. Each
chemotherapy at least 2 cycles
Exclusion criteria: no information
Participant (baseline) characteristics: main diagnosis: gastric cancer 18 people; lung cancer 17 peo-
ple; colorectal cancer 15 people; breast cancer 10 people
Setting and location: hospital, China
Number of people screened: 60


Lu 2013 (Continued)

\author{
Number of participants randomised - all: 60 \\ Number randomised to each group: 30 \\ Number receiving treatment as allocated: 60 \\ Number dropped out: 0 \\ Number excluded from analysis (for all outcomes): 0 \\ Number completed: 60
}

Interventions

Intervention group $\mathbf{A}(\mathrm{N}=30)$ : day 1 of chemotherapy given diphenhydramine $(20 \mathrm{mg} I \mathrm{M})+$ tropisetron ( $5 \mathrm{mg}$ IV) + dexamethasone (5-10 mg IV) until the end of chemotherapy, at the same time participants in treatment groups were given olanzapine ( $2.5 \mathrm{mg}$, twice/day, oral or $5 \mathrm{mg}$ once/day)

Control group C $(\mathrm{N}=30)$ : day 1 of chemotherapy given diphenhydramine (20 mg IM) + tropisetron (5 $\mathrm{mg}$ IV) + dexamethasone (5-10 mg IV) until the end of chemotherapy

Use of additional treatments if any: no information

\begin{tabular}{ll}
\hline Outcomes & Primary outcomes \\
& - Complete response \\
& Pecondary outcomes \\
& Mild response \\
& Others: compliance \\
\hline Funding & No information \\
\hline Declarations of Interest & No information \\
\hline Notes &
\end{tabular}

\title{
Risk of bias
}

Bias Authors' judgement Support for judgement

\begin{tabular}{lll}
\hline $\begin{array}{l}\text { Random sequence genera- } \\
\text { tion (selection bias) }\end{array}$ & Unclear risk & $\begin{array}{l}\text { Quote: "We adopt the dynamic randomized controlled method, patients were } \\
\text { randomly divided into two groups" } \\
\text { Comment: method of randomisation not described }\end{array}$ \\
\hline $\begin{array}{l}\text { Allocation concealment } \\
\text { (selection bias) }\end{array}$ & Unclear risk & $\begin{array}{l}\text { Quote: "We adopt the dynamic randomized controlled method, patients were } \\
\text { randomly divided into two groups" } \\
\text { Comment: allocation concealment not described }\end{array}$ \\
\hline
\end{tabular}

\begin{tabular}{|c|c|c|}
\hline $\begin{array}{l}\text { Blinding of participants } \\
\text { and personnel (perfor- }\end{array}$ & High risk & $\begin{array}{l}\text { Comment: no record of blinding of participants or assessors, but it is likely that } \\
\text { participants and personnel were not blinded though it was not described }\end{array}$ \\
\hline
\end{tabular}
mance bias)

All outcomes

$\begin{array}{ll}\text { Blinding of outcome as- } & \text { Unclear risk }\end{array}$

All outcomes

Incomplete outcome data Low risk $\quad$ Comment: all participants accounted for
(attrition bias)


Lu 2013 (Continued)

All outcomes

\begin{tabular}{|c|c|c|}
\hline $\begin{array}{l}\text { Selective reporting (re- } \\
\text { porting bias) }\end{array}$ & Unclear risk & $\begin{array}{l}\text { Comment: no protocol available on clincaltrials.gov or Chinese registry of clin- } \\
\text { ical trials (www.chictr.org.cn) }\end{array}$ \\
\hline
\end{tabular}

\begin{tabular}{lll}
\hline $\begin{array}{l}\text { Other bias (validation of } \\
\text { instruments) }\end{array}$ & Unclear risk & Comment: not mentioned \\
\hline Other bias & High risk & $<50$ participants per treatment arm
\end{tabular}

Mizukami 2014

\begin{tabular}{|c|c|}
\hline \multirow[t]{7}{*}{ Methods } & Trial method: RCT \\
\hline & Randomised: yes \\
\hline & Blinding: double-blinded \\
\hline & Arms: 2 \\
\hline & MEC or HEC: MEC/HEC \\
\hline & Multicentre/single-centre: 2 centres \\
\hline & Dates: unknown \\
\hline \multirow[t]{15}{*}{ Participants } & $N=48$ \\
\hline & Age: $22-78$ years \\
\hline & Gender: Male + Female \\
\hline & $\begin{array}{l}\text { Inclusion criteria: various cancers, planned to receive HEC or MEC, ECOG performance status } 0-2 \text {, no } \\
\text { significant nausea or vomiting or retching for } 24 \text { hour prior }\end{array}$ \\
\hline & $\begin{array}{l}\text { Exclusion criteria: vomiting, retching, or significant nausea in the } 24 \text { hours before the start of the trial, } \\
\text { were scheduled to receive concurrent abdominal radiation therapy, had a history of diabetes mellitus, } \\
\text { received treatment with other antipsychotic agents, or showed hypersensitivity to olanzapine }\end{array}$ \\
\hline & $\begin{array}{l}\text { Participant (baseline) characteristics: main diagnosis bladder, lymphoma, pharynx, breast, } \\
\text { leukaemia, others }\end{array}$ \\
\hline & Setting and location: hospital inpatients, Japan \\
\hline & Number of people screened: 48 \\
\hline & $\begin{array}{l}\text { Number of participants randomised all: } 48 \text { ( } 3 \text { had chemotherapy cancelled therefore did not go on to } \\
\text { receive treatment) }\end{array}$ \\
\hline & Number randomised to each group: 22 in control, 23 in intervention \\
\hline & Number receiving treatment as allocated: 45 \\
\hline & Number not receiving treatment as allocated: 0 \\
\hline & $\begin{array}{l}\text { Number dropped out: } 1 \text { from intervention arm due to drowsiness (no follow-up data for any outcome } \\
\text { available) }\end{array}$ \\
\hline & $\begin{array}{l}\text { Number excluded from analysis (for all outcomes): } 1 \text { from intervention arm dropped out of trial due to } \\
\text { drowsiness }\end{array}$ \\
\hline & Number completed: 43 total \\
\hline
\end{tabular}

Interventions

Intervention group A ( $\mathrm{N}=23)$ : dexamethasone, 5-HT3 antagonist and aprepitant prior then oral olanzapine $5 \mathrm{mg}$ daily on day 0 (day before chemotherapy) - 5, with $10 \mathrm{mg}$ IV metoclopramide as needed for rescue maximum 3 times/day

Control group B ( $\mathrm{N}=22)$ : dexamethasone, 5-HT3 antagonist and aprepitant prior then placebo on day 0 (day before chemotherapy)-5, with $10 \mathrm{mg}$ IV metoclopramide as needed for rescue maximum 3 times/ day

Use of additional treatments if any: 
Mizukami 2014 (Continued)

Outcomes
Outcomes of interest in the review

Primary outcomes

- The rate of participants who achieved total control (TC; no vomiting, no use of rescue medications, and maximum nausea of $5 \mathrm{~mm}$ on a $100 \mathrm{~mm}$ VAS) during the overall phase (0-120 hours), acute phase (0-24 hours), and delayed phase (24-120 hours)

Secondary outcomes

- QOL as assessed by the Japanese version of the FLI-E questionnaire, on day 0 and 6

- The satisfaction score (1: dislike; 2: not satisfied; 3: neither; 4: satisfied; 5: well satisfied)

- The rates of complete response (no vomiting and no use of rescue medications) and complete protection (complete response plus maximum VAS score)

Other outcomes reported by the study

The amount of dietary intake during chemotherapy

\begin{tabular}{ll}
\hline Funding & No information \\
\hline Declarations of Interest & None declared \\
\hline Notes &
\end{tabular}

\section{Risk of bias}

\begin{tabular}{lll}
\hline Bias & Authors' judgement & Support for judgement \\
\hline $\begin{array}{ll}\text { Random sequence genera- } \\
\text { tion (selection bias) }\end{array}$ & Unclear risk & $\begin{array}{l}\text { Quote: "After the screening for eligibility and registration in the study, patients } \\
\text { were randomly assigned to receive } 5 \mathrm{mg} / \text { day of oral olanzapine (OL group) or } \\
\text { placebo (control group) daily from the day before the start of chemotherapy } \\
\text { (Day 0) to Day 5." } \\
\text { Comment: method of randomisation not described }\end{array}$ \\
\end{tabular}

$\begin{array}{ll}\begin{array}{l}\text { Allocation concealment } \\ \text { (selection bias) }\end{array} & \text { Unclear risk } \\ & \text { were randomly assigned to receive } 5 \mathrm{mg} / \text { day of oral olanzapine (OL group) or } \\ & \text { placebo (control group) daily from the day before the start of chemotherapy } \\ \text { (Day 0) to Day 5." } & \text { Comment: method of allocation not described }\end{array}$

Blinding of participants Low risk and personnel (performance bias)

All outcomes
Quote: "Patients were blinded to the drug they were receiving." "To assess the antiemetic efficacy in the two groups, an investigator (N. M.), who was blinded to the drug being administered, recorded the following information every 24 hours for the first 120 hours after the initiation of HEC or MEC: use of any rescue medication for $\mathrm{CINV}$, the number and time of any emetic events, and maximum nausea experienced, which was rated using a visual analogue scale (VAS)."

Comment: the study was double-blinded

\section{Blinding of outcome as- Low risk} sessment (detection bias) All outcomes
Quote: "To assess the antiemetic efficacy in the two groups, an investigator ( $\mathrm{N}$. $\mathrm{M}$.), who was blinded to the drug being administered, recorded the following information every 24 hours for the first 120 hours after the initiation of HEC or MEC: use of any rescue medication for CINV, the number and time of any emetic events, and maximum nausea experienced, which was rated using a visual analogue scale (VAS)."
Quote: "48 patients from two institutions in Japan were randomly assigned to receive a six-day oral dose of either olanzapine or placebo. Three patients did not receive treatment (because of the cancellation of chemotherapy), and one 
patient from the OL group dropped out of the study because of drowsiness attributed to the study drug. Thus, 44 patients ( 22 in the OL group and 22 in the control group) were completely assessed."

Comment: no CONSORT diagram

\begin{tabular}{|c|c|c|}
\hline $\begin{array}{l}\text { Selective reporting (re- } \\
\text { porting bias) }\end{array}$ & Unclear risk & $\begin{array}{l}\text { Comment: no protocol available on clinicaltrials.gov or Japanese registry of } \\
\text { clinical trials (rctportal.niph.go.jp) }\end{array}$ \\
\hline
\end{tabular}

Other bias (validation of Low risk Validated VAS and FLIE scales

instruments)

Other bias High risk High risk of bias based on number of participants per arm $(n=22)$

Power calculation done

Trial method: RCT
Randomised: yes - computer-generated, simple randomisation
Blinding: participant and assessor blinded
Arms: 2
MEC or HEC: MEC/HEC
Multicentre/single-centre: single
Dates: not stated

Participants
Age: $55.04+/-1.50$ in control, $53.66+/-1.55$ in intervention
Gender: Male + Female
Inclusion criteria: histologically proven malignancy, due to receive a single day platinum-based
chemotherapy (carboplatin, cisplatin and oxaliplatin), chemotherapy-naive, $\geq 18-80$ years, acceptable
hepatic and renal function, no nausea or vomiting in 24 hours prior to starting treatment, neutrophil
count $>1500 / d L$
Exclusion criteria: seizures, unstable brain metastases, serious cardiac arrhythmia or dysfunction,
congestive heart failure, recent Ml, uncontrolled concurrent illness, uncontrolled diabetes, gastric out-
let obstruction, intestinal obstruction, known hypersensitivity to study drug, any chemotherapy within
previous 3 weeks, antiemetic steroid or other drug use that might affect study within previous 24 hours,
any antipsychotic use within previous 30 days, any other investigational drug use within previous 30
days, participation in any other study within 30 days prior to enrolment in study

Participant (baseline) characteristics: main diagnosis head and neck cancer, oesophageal cancer, cervical cancer, pancreatic cancer, ovarian cancer or other type

Setting and location: hospital, North West India

Number of people screened: 116

Number of participants randomised - all: 100

Number randomised to each group: 50

Number receiving treatment as allocated: 100

Number not receiving treatment as allocated: 0

Number excluded from analysis (for all outcomes): 0

Number completed: 100 
Mukhopadhyay 2016 (Continued)

Interventions

Intervention group $\mathbf{A}(\mathrm{N}=50)$ : olanzapine $10 \mathrm{mg}$ once/day, day 1-5, palonosetron $0.25 \mathrm{mg}$ IV once/day, dexamethasone IV $8 \mathrm{mg}$ in MEC or $16 \mathrm{mg} \mathrm{HEC}$ day 1 , then dexamethasone $8 \mathrm{mg}$ once/day, day $2+3$ in MEC or 8 mg twice/day, day $2+3$ in HEC

Control group $\mathbf{B}(\mathrm{N}=50)$ : palonosetron $0.25 \mathrm{mg}$ IV once/day, dexamethasone IV $8 \mathrm{mg}$ in MEC or $16 \mathrm{mg}$ HEC day 1 , then dexamethasone $8 \mathrm{mg}$ once/day, day $2+3$ in MEC or $8 \mathrm{mg}$ twice/day, day $2+3$ in HEC

Use of additional treatments if any: metoclopramide $10-20 \mathrm{mg}$ as needed oral or IV for breakthrough nausea and vomiting

Outcomes of interest in the review
Primary outcomes
- Complete response at day 1, day 2-5 and overall day 1-5
Secondary outcomes
- Control of nausea at day 1, day 2-5 and overall day 1-5 based on MASCC MAT score 2. Complete control
day 2-5
- Use of rescue medication day 1-5
- Q QOL measured day 8-10 using EORTC QLQ-C30

Other outcomes reported by the study: none

\begin{tabular}{ll}
\hline Funding & None declared \\
\hline Declarations of Interest & None declared \\
\hline Notes & \\
\hline
\end{tabular}

\section{Risk of bias}

\begin{tabular}{lll}
\hline Bias & Authors' judgement & Support for judgement \\
\hline $\begin{array}{ll}\text { Random sequence genera- } \\
\text { tion (selection bias) }\end{array}$ & Low risk & $\begin{array}{l}\text { Quote: "Patients were randomized by computer-generated simple randomiza- } \\
\text { tion." } \\
\text { Comment: from personal communication with AS. Adequate sequence gener- } \\
\text { ation }\end{array}$ \\
& &
\end{tabular}

$\begin{array}{ll}\begin{array}{l}\text { Allocation concealment } \\ \text { (selection bias) }\end{array} & \begin{array}{l}\text { Comment: although the text implies that the randomisation was done by com- } \\ \text { puter, it also notes that the clinicians weren't blind to the treatment group. It } \\ \text { is unclear whether they could have influenced the allocation }\end{array}\end{array}$

\begin{tabular}{|c|c|c|}
\hline $\begin{array}{l}\text { Blinding of participants } \\
\text { and personnel (perfor- } \\
\text { mance bias) }\end{array}$ & High risk & $\begin{array}{l}\text { Quote: "The enrolled patients ... were blinded about the treatment." } \\
\text { "The clinician was not blinded and therefore possibility of bias could not be } \\
\text { excluded." }\end{array}$ \\
\hline
\end{tabular}

All outcomes

Comment: from personal communication with AS. Although the study comments that the participants were blinded to treatment group there was no placebo treatment given in the control arm. In addition the paper notes that clinicians were not blinded to the treatment group. This could have possibly affected the treatment given

\begin{tabular}{|c|c|c|}
\hline $\begin{array}{l}\text { Blinding of outcome as- } \\
\text { sessment (detection bias) } \\
\text { All outcomes }\end{array}$ & Unclear risk & $\begin{array}{l}\text { Quote: "The enrolled patients ... blinded about the treatment." } \\
\text { "Vomiting diary was used by the patients to report number and time of emetic } \\
\text { episodes in the first } 24 \text { hours and then everyday up to } 5 \text { days." } \\
\text { "Patients were reassessed between } 8 \text { th and } 10 \text { th days after chemotherapy and } \\
\text { data collection was done by a blinded assessor, a trained nurse blinded of the } \\
\text { treatment." }\end{array}$ \\
\hline
\end{tabular}


"The clinician was not blinded and therefore possibility of bias could not be excluded."

Comment: although the paper states that assessors were blinded to treatment group the diaries were kept by the participants who were stated as blinded but potential for bias exists as there was no placebo given in the control group. It was unclear if these methods would have influenced the assessment of the outcomes.

\begin{tabular}{ll}
$\begin{array}{l}\text { Incomplete outcome data } \\
\text { (attrition bias) }\end{array}$ & $\begin{array}{l}\text { Comment: no participants were lost to follow-up. All participants were } \\
\text { All outcomes }\end{array}$ \\
\hline
\end{tabular}

All outcomes

Comment: no protocol available on clinicaltrials.gov or Indian clinical trials registry (ctri.nic.in)

Selective reporting (re- Unclear risk porting bias)

Comment: paper discusses validation of the EORTC QLQ-C30 and tool for as-

Other bias (validation of Low risk instruments) sessing control of nausea and vomiting (MASCC) scale

Other bias Unclear risk Unclear risk of bias based on number of participants per arm $(n=50)$

Nakagaki 2017

\begin{tabular}{|c|c|}
\hline \multirow[t]{7}{*}{ Methods } & Trial method: RCT \\
\hline & Randomised: yes \\
\hline & Blinding: no, open-label \\
\hline & Arms: 3 \\
\hline & MEC or HEC: HEC \\
\hline & Multicentre/single-centre: single \\
\hline & Dates: not stated \\
\hline \multirow[t]{13}{*}{ Participants } & $N=62$ \\
\hline & Age: $20-68$ years \\
\hline & Gender: Male + Female \\
\hline & $\begin{array}{l}\text { Inclusion criteria: HSCT patients suffering breakthrough CINV during allogenic or autogenic trans- } \\
\text { plant following chemotherapy, despite standard prophylaxis with IV ondansetron } 8 \mathrm{mg} 3 \text { times/day } \\
\text { plus a single dose of oral aprepitant } 165 \mathrm{mg} \text {, age }>18 \text { and }<70 \text {, breakthrough nausea and vomiting was } \\
\text { defined as a VAS } \geq 3 \text {, or requiring }>1 \times \text { as needed/day for nausea or vomiting }\end{array}$ \\
\hline & $\begin{array}{l}\text { Exclusion criteria: "Patients were excluded if they were allergic to any of the study medications, tak- } \\
\text { ing olanzapine as a regular medication, at risk for an adverse drug event from the study drugs (e.g. pa- } \\
\text { tients with QT prolongation), had nausea or vomiting before HSCT, or did not have an adequate under- } \\
\text { standing of written and spoken English." }\end{array}$ \\
\hline & Participant (baseline) characteristics: main diagnosis: all undergoing autologous or allogeneic HSCT \\
\hline & $\begin{array}{l}\text { Other important effect modifiers, if applicable (e.g. radiotherapy): all participants received chemother- } \\
\text { apy but some also received total body irradiation (TBI) }\end{array}$ \\
\hline & Setting and location: inpatient bone marrow transplant centre, Australia \\
\hline & Number of people screened: 94 \\
\hline & $\begin{array}{l}\text { Number of participants randomised - all: } 62 \text { at the point where breakthrough nausea or vomiting oc- } \\
\text { curred }\end{array}$ \\
\hline & $\begin{array}{l}\text { Number randomised to each group: 18, } 22 \text { and } 22 \text { patients were randomised to the ondansetron, olan- } \\
\text { zapine and palonosetron arms }\end{array}$ \\
\hline & Number receiving treatment as allocated: 62 \\
\hline & Number not receiving treatment as allocated: 0 \\
\hline
\end{tabular}


Nakagaki 2017 (Continued)

Number dropped out: 0 (no follow-up data for any outcome available)

Number excluded from analysis (for all outcomes): 0

Number completed: 62

$\begin{array}{ll}\text { Interventions } & \text { Intervention group A }(\mathrm{N}=22) \text { : olanzapine } 10 \mathrm{mg} \text { once/day with IV ondansetron } 8 \mathrm{mg} 3 \text { times/day } \\ & \text { Intervention group B ( }=18) \text { : ondansetron } 32 \mathrm{mg} \text { in } 250 \mathrm{~mL} \text { normal saline as a continuous infusion } \\ \text { over } 24 \text { hours } \\ \text { Intervention group C }(\mathrm{N}=22) \text { : palonosetron } 0.25 \mathrm{mg} \mathrm{IV} \mathrm{immediately,} \mathrm{no} \mathrm{further} \mathrm{ondansetron} \mathrm{for} 3 \\ \text { days }\end{array}$

Use of additional treatments if any: all groups were allowed as-needed metoclopramide $10 \mathrm{mg}$ oral or IV and/or lorazepam $1 \mathrm{mg}$ sublingual as rescue antinausea medication. "immunosuppressants, anti-infectives, nutritional supplementation, and a proton pump inhibitor. Steroids were not used as antiemetics but allowed to be prescribed to prevent hypersensitivity with drugs and blood products."

\begin{tabular}{|c|c|c|}
\hline Outcomes & $\begin{array}{l}\text { Outcomes of interest } t \\
\text { Primary outcomes } \\
\text { - } 1 . \text { SAEs } \\
\text { Secondary outcomes } \\
\text { - No emesis, no use } \\
48 \text { hours } \\
\text { - AEs } \\
\text { - Withdrawals }\end{array}$ & rescue medication, and nausea score reduction of $>50 \%$ on VAS at 24 hours and \\
\hline Funding & \multicolumn{2}{|c|}{$\begin{array}{l}\text { "This study was financially supported in part by the Royal Brisbane and Women's Hospital Founda- } \\
\text { tion." }\end{array}$} \\
\hline Declarations of Interest & \multicolumn{2}{|c|}{ "It was also conducted as dissertation in Master of Oncology, Newcastle University, UK." } \\
\hline Notes & \multicolumn{2}{|c|}{ We combined the ondansetron and palonosetron arms together to create one '5-HT3 antagonist' arm } \\
\hline \multicolumn{3}{|l|}{ Risk of bias } \\
\hline Bias & Authors' judgement & Support for judgement \\
\hline $\begin{array}{l}\text { Random sequence genera- } \\
\text { tion (selection bias) }\end{array}$ & Low risk & $\begin{array}{l}\text { Quote: from personal correspondence between AS and the study author: } \\
\text { "...patients were randomized in a 1:1:1 fashion into one of the three treatment } \\
\text { arms..." "...we used opaque sealed envelopes with indication of one arm in it } \\
\text { and it was randomly picked by treating nurses." "The opaque envelops were } \\
\text { shuffled and placed at random. The nurses were instructed to pick at random } \\
\text { from the box." } \\
\text { Comment: method of randomisation as the envelopes randomly placed within } \\
\text { the box }\end{array}$ \\
\hline $\begin{array}{l}\text { Allocation concealment } \\
\text { (selection bias) }\end{array}$ & Low risk & $\begin{array}{l}\text { Quote: From personal correspondence between AS and the author: "...we used } \\
\text { opaque sealed envelopes with indication of one arm in it and it was randomly } \\
\text { picked by treating nurses." } \\
\text { Comment: envelopes picked randomly from box without knowledge of study } \\
\text { arm }\end{array}$ \\
\hline $\begin{array}{l}\text { Blinding of participants } \\
\text { and personnel (perfor- } \\
\text { mance bias) } \\
\text { All outcomes }\end{array}$ & High risk & Quote: "A randomized open-label prospective study ..." \\
\hline
\end{tabular}


Nakagaki 2017 (Continued)

Blinding of outcome assessment (detection bias) All outcomes
High risk

Quote: "Assessment of emesis events and severity of nausea were documented by patients, while rescue antiemetic usage was obtained from the medication administration record."

"To quantify the severity of nausea, patients were asked to use the $100 \mathrm{~mm}$ VAS on a data collection sheet..."

Comment: low-risk method but in the context of unblinded participants and personnel represents a high risk of bias

Incomplete outcome data Low risk Comment: data reported for 62 out of 64 participants who were randomised
(attrition bias)

All outcomes

\begin{tabular}{lll}
\hline $\begin{array}{l}\text { Selective reporting (re- } \\
\text { porting bias) }\end{array}$ & Unclear risk & $\begin{array}{l}\text { Comment: no protocol available or found on ClinicalTrials.gov or Australian } \\
\text { clinical trials registry (www.anzctr.org.au/) }\end{array}$ \\
\hline $\begin{array}{l}\text { Other bias (validation of } \\
\text { instruments) }\end{array}$ & Low risk & Validated VAS score used \\
\hline
\end{tabular}

Other bias High risk High risk of bias based on number of participants per arm $(18,22,22)$

Navari 2010b

$\begin{array}{ll}\text { Methods } & \text { Trial method: RCT } \\ & \text { Randomised: consecutive randomisation } \\ \text { Blinding: unblinded } & \text { Arms: } 2 \\ \text { MEC or HEC: } \text { not receiving chemotherapy } \\ \text { Multicentre/single-centre: single } \\ \text { Dates: } 31 \text { March 2005-31 December } 2007\end{array}$

Participants $\quad \mathrm{N}=80$

Age: $39-81$ years

Gender: Male + Female

Inclusion criteria: > 18 years, histologically or cytologically proven gastrointestinal or lung cancer stage III-IV, anorexia, cancer-related loss of appetite, $\geq 5 \%$ weight loss, stable renal function, hepatic function and neutrophil count $\geq 1500 \mathrm{~mm}^{2}$, agree to use of contraception if of childbearing age, negative pregnancy test for women of childbearing age, could use prochlorperazine or phenothiazines as antiemetics if needed

Exclusion criteria: no chemotherapy, radiotherapy or surgery in last 4 weeks, no dysphagia or gastrointestinal obstruction, no thrombophlebitis, no steroid us in past 4 weeks, no severe cognitive impairment, no antipsychotic use 30 days prior or during protocol, no alcoholism, no use of ethyol, no concurrent abdominal radiotherapy, no quinolone antibiotics, no hypersensitivity to olanzapine, no cardiac arrhythmia, CCF or MI in past 6 months, no uncontrolled diabetes mellitus

Participant (baseline) characteristics: main diagnosis lung cancer stage III or IV, colon cancer stage III or IV

Setting and location: cancer centre, USA

Number of people screened: 80

Number of participants randomised - all: 80 
Navari 2010b (Continued)

Number randomised to each group: 40

Number receiving treatment as allocated: 37 in MA arm, 39 in MA + olanzapine

Number not receiving treatment as allocated: 4 withdrawals 1 of these died in MA arm

- reason 1 - MI, CCF

- reason 2 - use of quinolone, non-compliance went on to die 2 weeks later of disease progression

Number dropped out: 0

Number excluded from analysis (for all outcomes): 0

Number completed: 76

\begin{tabular}{|c|c|}
\hline Interventions & $\begin{array}{l}\text { Intervention group } \mathbf{A}(\mathrm{N}=40) \text { : megestrol acetate } 800 \mathrm{mg} / \text { day and olanzapine } 5 \mathrm{mg} / \text { day for } 8 \text { weeks } \\
\text { Intervention group } \mathbf{B}(\mathrm{N}=40) \text { : megestrol acetate } 800 \mathrm{mg} / \text { day for } 8 \text { weeks } \\
\text { Use of additional treatments if any (co-interventions, additional analgesia) }\end{array}$ \\
\hline Outcomes & $\begin{array}{l}\text { Outcomes of interest in the review } \\
\text { Primary outcomes } \\
\text { - 3-point improvement in nausea on VAS } \\
\text { - QOL at } 4 \text { and } 8 \text { weeks } \\
\text { Other outcomes reported by the study: } \\
\text { - } \geq 5 \% \text { weight gain } \\
\text { - 3-point improvement in appetite on VAS }\end{array}$ \\
\hline Funding & Walther Cancer Foundation and Reich Family Endowment \\
\hline Declarations of Interest & No information \\
\hline
\end{tabular}

\section{Risk of bias}

\begin{tabular}{|c|c|c|}
\hline Bias & Authors' judgement & Support for judgement \\
\hline $\begin{array}{l}\text { Random sequence genera- } \\
\text { tion (selection bias) }\end{array}$ & Unclear risk & $\begin{array}{l}\text { Quote: "Patients eligible for the study were consecutively randomised..." } \\
\text { Comment: no randomisation method stated }\end{array}$ \\
\hline $\begin{array}{l}\text { Allocation concealment } \\
\text { (selection bias) }\end{array}$ & Unclear risk & Quote: "Patients eligible for the study were consecutively randomised..." \\
\hline $\begin{array}{l}\text { Blinding of participants } \\
\text { and personnel (perfor- } \\
\text { mance bias) } \\
\text { All outcomes }\end{array}$ & High risk & $\begin{array}{l}\text { Quote: "Patients...receive oral MA at a dose of } 800 \mathrm{mg} / \mathrm{day} \text { or oral MA at } 800 \\
\mathrm{mg} / \text { day plus OLN at } 5 \mathrm{mg} / \text { day." } \\
\text { "...the treatment arms were not blinded..." "....the treatment arms were not } \\
\text { blinded, and a placebo effect cannot be eliminated in the study." } \\
\text { Comment: different number of tablets per arm }\end{array}$ \\
\hline $\begin{array}{l}\text { Blinding of outcome as- } \\
\text { sessment (detection bias) } \\
\text { All outcomes }\end{array}$ & High risk & $\begin{array}{l}\text { Quote: "...patients were asked to record..." } \\
\text { "A nurse/research coordinator contacted each patient each week to remind } \\
\text { the patient to complete the forms and to query toxicities. Patients were seen } \\
\text { and examined by their physician every } 2 \text { weeks during the study period." } \\
\text { Comment: low-risk method but in the context of unblinded participants self- } \\
\text { reporting and unblinded personnel this represents a high risk of bias }\end{array}$ \\
\hline
\end{tabular}


Navari 2010b (Continued)

Incomplete outcome data Low risk Comment: 80 participants randomised, 76 participants evaluable, 4 partici(attrition bias) pants lost due to attrition but clear reasons for each

All outcomes

Selective reporting (re- Unclear risk Comment: no protocol available on clinicaltrials.gov
porting bias)

Other bias (validation of Low risk Used validated scales: VAS, MD Anderson, FACT-3

instruments)

Other bias High risk High risk of bias based on number of participants per arm $(n=40)$

Navari 2013b

Trial method: RCT
Randomised: computer-generated random assignment schedule created by a statistician not involved
with the study immediately after chemotherapy
Blinding: double-blind
Arms: 2
MEC or HEC: HEC
Multicentre/single-centre: 3
Dates: not stated

Participants

$N=280$

Age: $38-79$

Gender: Male + Female

Inclusion criteria: eligible patients were $>18$ years of age with histologically or cytologically confirmed malignant disease that were chemotherapy naive and scheduled to receive HEC (cisplatin, $\geq 70 \mathrm{mg} /$ $\mathrm{m} 2$; cyclophosphamide, $\geq 600-1000 \mathrm{mg} / \mathrm{m} 2$; and doxorubicin, $\geq 50-60 \mathrm{mg} / \mathrm{m} 2$ ). Serum creatinine of $\leq 2.0 \mathrm{mg} / \mathrm{dL}$, serum bilirubin of $\leq 2.0 \mathrm{mg} / \mathrm{dL}$, SGOT or SGPT values of $\leq 3$ times the upper limits of normal, and absolute neutrophil count of $\geq 1,500 \mathrm{~mm} 3$. Participants of childbearing potential (men and women) had to consent to use adequate contraception throughout protocol therapy. Women of childbearing potential had to have a negative urine pregnancy test, may receive prochlorperazine and other phenothiazines as rescue antiemetic therapy

Exclusion criteria: no nausea and vomiting for 24 hours, no severe cognitive compromise, no known history of CNS disease (e.g. brain metastases, seizure disorder), no treatment with other antipsychotic agents such as risperidone, quetiapine, clozapine, phenothiazine or butyrophenone for 30 days prior to or during protocol therapy. Patients on chronic phenothiazine administration as an antipsychotic agent not allowed. No concurrent use of ethyol, no concurrent abdominal radiotherapy, no concurrent use of quinolone antibiotic therapy, no chronic alcoholism (as determined by the investigator), no known hypersensitivity to olanzapine, no known cardiac arrhythmia, uncontrolled CHF, or acute MI within the previous 6 months, and no history of uncontrolled diabetes mellitus

Participant (baseline) characteristics: main diagnosis bladder cancer, breast cancer, non-small cell lung cancer and lymphoma

Setting and location: outpatient oncology centres, USA

Number of people screened: 280

Number of participants randomised - all: 276

Number randomised to each group: 138 per arm 
Number not receiving treatment as allocated: 80 in olanzapine and 84 in metoclopramide group as did not develop breakthrough CINV

Number dropped out: 4; 3 discontinued intervention, 1 lost to follow-up (no follow-up data for any outcome available)

Number excluded from analysis (for all outcomes): 0

Number completed: 56 olanzapine, 52 metoclopramide

Interventions Intervention group A ( $\mathrm{N}=58)$ : olanzapine $10 \mathrm{mg}$ once/day, day 1-3 from point of developing breakthrough CINV

Intervention group B ( $\mathrm{N}=54)$ : metoclopramide $10 \mathrm{mg} 3$ times/day, day 1-3 from point of developing breakthrough CINV

Use of additional treatments if any: day 1 dexamethasone $12 \mathrm{mg} \mathrm{IV}$, palonosetron $0.25 \mathrm{mg}$ IV, fosaprepitant $150 \mathrm{mg} \mathrm{IV}$, day 2-4 $8 \mathrm{mg}$ oral dexamethasone once/day but this was stopped if breakthrough nausea or vomiting occurred and the intervention commenced instead

Outcomes Outcomes of interest in the review

Primary outcomes

- Number of participants with no emetic episodes in the 72 hour observation

- Number of participants with no nausea in the 72 hour observation period

Secondary outcomes:

- SAES

- Other AEs

Other outcomes reported by the study: none

\begin{tabular}{ll}
\hline Funding & Reich Family Endowment for Care of the Whole Patient \\
\hline Declarations of Interest & None declared \\
\hline Notes & \\
\hline
\end{tabular}

\section{Risk of bias}

\begin{tabular}{lll}
\hline Bias & Authors' judgement & Support for judgement \\
\hline $\begin{array}{ll}\text { Random sequence genera- } \\
\text { tion (selection bias) }\end{array}$ & Low risk & $\begin{array}{l}\text { Quote: "All patients eligible for the study were randomized to either the olan- } \\
\text { zapine treatment regimen or the metoclopramide treatment regimen accord- } \\
\text { ing to a computer-generated random assignment schedule created by a statis- } \\
\text { tician not involved with the study." }\end{array}$ \\
& &
\end{tabular}

\begin{tabular}{lll}
\hline $\begin{array}{l}\text { Allocation concealment } \\
\text { (selection bias) }\end{array}$ & Unclear risk & Comment: method of allocation was not described \\
\hline $\begin{array}{l}\text { Blinding of participants } \\
\begin{array}{l}\text { and personnel (perfor- } \\
\text { mance bias) }\end{array}\end{array}$ & Low risk & $\begin{array}{l}\text { Quote: "The treatment packages were identical with neither the patients nor } \\
\text { the investigators knowing which treatment the patients were assigned." } \\
\text { All outcomes }\end{array}$ \\
\hline
\end{tabular}

\begin{tabular}{lll}
\hline Blinding of outcome as- & Low risk & $\begin{array}{l}\text { Quote: "Beginning with the initiation of the breakthrough treatment regimen } \\
\text { and continuing for } 72 \text { hours, patients were asked to record episodes of vom- } \\
\text { sessment (detection bias) }\end{array}$ \\
All outcomes & iting/retching (number and time) and the daily intensity of symptoms utiliz-
\end{tabular}


Navari 2013b (Continued)

ing the MDASI. Patients were also asked to record daily episodes of nausea using a visual analogue scale from 0 to 10 , with 0 indicating no nausea and $10 \mathrm{in}-$ dicating a maximal level of nausea. A nurse/research coordinator contacted each patient every 24 hours to remind the patient to complete the forms and to query toxicities."

Comment: low-risk method, blinded participants and personnel

$\begin{array}{ll}\begin{array}{l}\text { Incomplete outcome data } \\ \text { (attrition bias) }\end{array} & \text { Low risk } \\ \text { All outcomes } & \begin{array}{l}\text { Comment: randomised before chemotherapy but only those participants who } \\ \text { developed breakthrough nausea and vomiting were eligible to receive inter- } \\ \text { vention. Unusual design but only attrition following this point analysed due to } \\ \text { design. }\end{array}\end{array}$

Data were not available for 4/112 (4\%) participants.

\begin{tabular}{lll}
\hline $\begin{array}{l}\text { Selective reporting (re- } \\
\text { porting bias) }\end{array}$ & Unclear risk & Comment: no protocol available on ClinicalTrials.gov \\
\hline $\begin{array}{l}\text { Other bias (validation of } \\
\text { instruments) }\end{array}$ & Low risk & Comment: study used VAS and MD Anderson \\
\hline Other bias & Unclear risk & $\begin{array}{l}\text { Comment: unclear risk of bias based on number of participants per arm (54 } \\
\text { and 58) }\end{array}$ \\
\hline
\end{tabular}

Navari $2016 a$

\begin{tabular}{ll}
\hline Methods & Trial method: RCT \\
& Randomised: yes using Pocock and Simon dynamic randomisation procedure, which balances the mar- \\
ginal distributions of the stratification factors between study groups, not stated if computer-generat- \\
ed/remote, etc \\
Blinding: double-blind \\
Arms: 2 \\
MEC or HEC: HEC \\
Multicentre/single-centre: 46 \\
Dates: August 2014 - March 2015
\end{tabular}

Participants $\quad \mathrm{N}=401$

Age: $28-89$ years

Gender: Male + Female

Inclusion criteria: patients $\geq 18$ years with malignant disease who had not received previous chemotherapy, HEC, ECOG performance status of 0,1 , or 2 , creatinine level of $\leq 2.0 \mathrm{mg} / \mathrm{dL}(177 \mu \mathrm{mol} / \mathrm{L})$, an aspartate or alanine aminotransferase level that was $\leq 3$ times the upper limit of the normal range, and an absolute neutrophil count of $\geq 1500 / \mathrm{mm} 3$, women of childbearing age had to have a negative result of a pregnancy test performed within 7 day before enrolment and agree to use appropriate birth control throughout their participation in the study.

Exclusion criteria: nausea or vomiting in the 24 hours before enrolment, severe cognitive compromise; no known history of CNS disease (e.g. brain metastases or a seizure disorder), treatment with another antipsychotic agent such as risperidone, quetiapine, clozapine, a phenothiazine, or a butyrophenone within 30 days before enrolment or plans for such treatment during the study period, long-term use of a phenothiazine as an antipsychotic agent, concurrent use of amifostine, concurrent abdominal radiotherapy; concurrent use of quinolone antibiotic therapy; chronic alcoholism; known hypersensitivity to olanzapine; known cardiac arrhythmia, uncontrolled CHF, or acute MI within the previous 6 months; history of uncontrolled diabetes mellitus 
Participant (baseline) characteristics: main diagnosis: breast cancer, lung cancer, other

Setting and location: 46 academic or community practice institutions in the USA

Number of people screened: 401

Number of participants randomised - all: 401

Number randomised to each group: 202 olanzapine, 199 placebo

Number receiving treatment as allocated:192 olanzapine, 188 placebo

Number not receiving treatment as allocated:

- olanzapine arm: 10 were excluded; 8 withdrew, 2 had major violations

- placebo arm: 11 were excluded; 10 withdrew, 1 had a major violation

Number dropped out: (no follow-up data for any outcome available )

Number excluded from analysis (for all outcomes):

- olanzapine arm: 6 were excluded owing to lack of nausea data

- placebo arm: 5 were excluded owing to a lack of nausea data

Number completed:

- olanzapine arm: 186

- placebo arm: 183

Intervention group A ( N = 202): $10 \mathrm{mg}$ of olanzapine orally
Control group B $(\mathrm{N}=199)$ : placebo
Use of additional treatments if any (co-interventions, additional analgesia): all participants received
a 5-HT3-receptor antagonist (palonosetron IV at a dose of $0.25 \mathrm{mg}$, granisetron IV at a dose of $1 \mathrm{mg}$ or
orally at a dose of $2 \mathrm{mg}$, or ondansetron IV or orally at a dose of $8 \mathrm{mg}$, with the specific agent chosen by
the primary clinician) on day 1 of chemotherapy, dexamethasone (12 mg orally on day 1 , and $8 \mathrm{mg}$ oral-
ly on day 2, 3, and 4), and an NK1-receptor antagonist on day 1 . Rescue antiemetics for breakthrough
nausea and vomiting were at the physician's choice. Since this is a double-blind trial it is possible the
additional doses of olanzapine may have been given

Outcomes Primary outcomes

- Complete response (no emetic episodes and no use of rescue medication) on the basis of the participants' daily records during the overall, early, and later assessment periods

Secondary outcomes

- No nausea, was defined as a response of 0 on the VAS for nausea during the overall assessment period (0-120 hours), the early assessment period (0-24 hours), and the later assessment period (25-120 hours)

- AEs

\begin{tabular}{ll}
\hline Funding & No information \\
\hline Declarations of Interest & No information \\
\hline Notes & \\
\hline Risk of bias & Authors' judgement $\quad$ Support for judgement \\
\hline Bias & \\
\hline
\end{tabular}


Navari 2016a (Continued)

Random sequence genera- Low risk tion (selection bias)
Quote: "Patients were assigned to a study group with the use of the Pocock and Simon dynamic randomization procedure, which balances the marginal distributions of the stratification factors between study groups. The stratification factors were sex, chemotherapy regimen (cisplatin-containing regimen vs. anthracycline plus cyclophosphamide), and the specific 5-HT3-receptor antagonist used (palonosetron, ondansetron, or granisetron)."

\begin{tabular}{|c|c|c|}
\hline $\begin{array}{l}\text { Allocation concealment } \\
\text { (selection bias) }\end{array}$ & Low risk & $\begin{array}{l}\text { Quote: "The patients and the medical professionals who cared for them were } \\
\text { unaware of the assigned study regimen." }\end{array}$ \\
\hline
\end{tabular}

\begin{tabular}{|c|c|c|}
\hline $\begin{array}{l}\text { Blinding of participants } \\
\text { and personnel (perfor- } \\
\text { mance bias) } \\
\text { All outcomes }\end{array}$ & Low risk & $\begin{array}{l}\text { Quote: "... patients received olanzapine ( } 10 \mathrm{mg} \text { per day orally) or a matching } \\
\text { placebo on days } 1 \text { through } 4 . " \\
\text { "The patients and the medical professionals who cared for them were un- } \\
\text { aware of the assigned study regimen." } \\
\text { Comment: this trial was double-blinded }\end{array}$ \\
\hline $\begin{array}{l}\text { Blinding of outcome as- } \\
\text { sessment (detection bias) } \\
\text { All outcomes }\end{array}$ & Low risk & $\begin{array}{l}\text { Quote: "Patients were asked to complete daily records of episodes of vomit- } \\
\text { ing or retching (number and time) and the use of rescue therapy from the first } \\
\text { day of chemotherapy (day } 1 \text { ) through day } 5 \text {. Patients were also asked to record } \\
\text { daily levels of nausea according to a visual-analogue scale } 24 \text { ranging from } 0 \\
\text { ("no nausea at all") to } 10 \text { ("nausea as bad as it can be"). A study nurse contact- } \\
\text { ed each patient daily on days } 2 \text { through } 5 \text { to ask about toxic effects and remind } \\
\text { the patient to complete forms." } \\
\text { Comment: self-reporting is a low-risk method in the context of blinded partici- } \\
\text { pants }\end{array}$ \\
\hline
\end{tabular}

\begin{tabular}{|c|c|c|}
\hline $\begin{array}{l}\text { Incomplete outcome data } \\
\text { (attrition bias) }\end{array}$ & High risk & $\begin{array}{l}\text { Comment: } 32 \text { participants had no evaluable data out of a total of } 401 \text { partici- } \\
\text { pants who were randomised ( } 17.9 \% \text { attrition rate) }\end{array}$ \\
\hline
\end{tabular}
All outcomes

Comment: 32 participants had no evaluable data out of a total of 401 partici-

Selective reporting (re- High risk
porting bias)

Comment: all intended outcomes were not reported. "Frequency of Rescue Medication (Time Frame: day 2 to day 6 after chemotherapy)" stipulated in the protocol NCT02116530 (ClinicalTrials.gov) was not reported. Additionally, there was discrepancy between CONSORT participant numbers and numbers in results table

\begin{tabular}{lll}
\hline $\begin{array}{l}\text { Other bias (validation of } \\
\text { instruments) }\end{array}$ & Low risk & Validated VAS scale used \\
\hline Other bias & Low risk & Low risk of bias based on number of participants per arm (202 and 199) \\
\hline
\end{tabular}

Nikbakhsh 2016

\begin{tabular}{ll}
\hline Methods & Trial method: $\mathrm{RCT}$ \\
& Randomised: yes \\
& MEC or HEC: unclear \\
& Blinding: unblinded \\
Arms: 2 \\
Multicentre/single-centre: single centre \\
Dates: not stated \\
N=30 \\
Age: mean 61.57 years \\
Gender: not provided
\end{tabular}


Nikbakhsh 2016 (Continued)

Inclusion criteria: gastric cancer was diagnosed in the previous month. Referring to the Oncology Department affiliated to Babol University of Medical Sciences. Physician's planning on chemotherapy treatment protocol for the patient. Informed consent of the patient for participation in the study Exclusion criteria: major psychiatric disorders such as schizophrenia, bipolar disorder, and dementia. Use of other antipsychotic drugs such as risperidone, clozapine, and phenothiazine in 30 day before the protocol beginning. History of severe neurologic problems such as brain metastases, convulsion, and mental retardation. Serum creatinine level $>2 \mathrm{mg} / \mathrm{dL}$. Serum bilirubin level $>2 \mathrm{mg} / \mathrm{dL}$. Serum glutamic oxaloacetic transaminase and serum glutamic pyruvic transaminase $>3$ times above the normal range ("normal range of our laboratory was $<30 \mathrm{U} / \mathrm{L}$ in women and $<40 \mathrm{U} / \mathrm{L}$ in men"). Neutrophil count $<1500 / \mathrm{mm} 3$. Pregnancy. Uncontrolled diabetes mellitus (fasting blood glucose $\geq 126 \mathrm{mg} / \mathrm{dL}$ ). Uncontrolled severe cardiac problems such as arrhythmias, CHF, and acute MI during the preceding 6 months Baseline characteristics:

Setting and location: University hospital, Babol, Iran

Number of people screened: 30

Number of participants randomised - all: 30

Number randomised to each group: Group A: 15; Group B: 15

Number receiving treatment as allocated: Group A: 15; Group B: 15

Number not receiving treatment as allocated: Group A: 0; Group B: 0

Number dropped out: Group A: 0; Group B: 0 (no follow-up data for any outcome available)

Number excluded from analysis (for all outcomes): Group A: 0; Group B: 0

Number completed: Group A: 15; Group B: 15

Intervention group $\mathbf{A}(\mathrm{N}=15)$ : day 1 - 8 weeks olanzapine $2.5 \mathrm{mg}$ once/day up to maximum dose of olanzapine $10 \mathrm{mg}$ once/day, based on participant tolerance and usual care

Control group $\mathbf{B}(\mathrm{N}=15)$ : usual care

Use of additional treatments if any (co-interventions, additional analgesia): routine treatment (not stated what this was)

\begin{tabular}{ll}
\hline Outcomes & Primary outcomes \\
& - Nausea and vomiting evaluated by Rhodes Index. day 0-5 \\
& Secondary outcomes \\
& - Patient perspective of treatment. HADS during 1st, 4th and 8th week WHO-QOL-BREF questionnaire \\
\hline Funding & "This study was supported by Babol University of Medical Sciences, Iran (Research Project Number: \\
\hline Declarations of Interest & "There are no conflicts of interest" \\
\hline Notes &
\end{tabular}

\section{Risk of bias}

\begin{tabular}{lll}
\hline Bias & Authors' judgement & Support for judgement \\
\hline $\begin{array}{l}\text { Random sequence genera- } \\
\text { tion (selection bias) }\end{array}$ & Unclear risk & $\begin{array}{l}\text { Quote: "The patients were allocated to two groups by simple random sam- } \\
\text { pling." } \\
\text { Comment: the paper does not provide any further information }\end{array}$ \\
\hline $\begin{array}{l}\text { Allocation concealment } \\
\text { (selection bias) }\end{array}$ & Unclear risk & $\begin{array}{l}\text { Quote: "The patients were allocated to two groups by simple random sam- } \\
\text { pling." } \\
\text { Comment: there is no information about the allocation concealment in the } \\
\text { paper }\end{array}$ \\
\hline
\end{tabular}

Blinding of participants and personnel (performance bias)
High risk

Comment: it does not appear that any attempts were made to blind participants or healthcare professionals to the treatment group 
Nikbakhsh 2016 (Continued)

All outcomes

Blinding of outcome as- High risk sessment (detection bias) All outcomes
Quote: "The patients were followed from the 1 st ( 0 day) to the 5 th day after chemotherapy for detection of N/V; in the 1st, 4th, and 8th week after intervention for Hospital Anxiety and Depression Scale (HADS) assessment; and 8 weeks after chemotherapy for patient's tolerance and adverse reactions. In these follow-ups, the patients' appetite and other physical symptoms were evaluated with a physician visit."

Comment: it is unclear whether participants self-reported any of the outcomes or whether the physician assessed the outcomes. However, as neither were blinded the risk of detection bias is high

$\begin{aligned} & \text { Incomplete outcome data Low risk } \\ & \begin{array}{l}\text { (attrition bias) } \\ \text { All outcomes }\end{array}\end{aligned}$
trial

\begin{tabular}{ll}
\hline $\begin{array}{l}\text { Selective reporting (re- } \\
\text { porting bias) }\end{array}$ & Low risk \\
& $\begin{array}{l}\text { Comment: clinical trial protocol is available through the Iranian Registry of } \\
\text { Clinical Trials (IRCT2015070822991N2). The outcomes presented in the proto- } \\
\text { col are reported in the paper }\end{array}$
\end{tabular}

Other bias (validation of Low risk Good description of the validation of the instruments used in the trial.
instruments)

Other bias High risk 15 participants per treatment arm

Shumway 2015

\begin{tabular}{ll}
\hline Methods & Trial method: RCT \\
& Randomised: yes \\
& MEC or HEC: HEC \\
Blinding: double-blind \\
Arms: 2 \\
Multicentre/single-centre: single \\
Dates: not stated
\end{tabular}

Participants

\author{
$\mathrm{N}=20(10$ per $\mathrm{arm})$ \\ Age: median, olanzapine: 54 years; aprepitant: 60.5 years \\ Gender: Male + Female
}

Inclusion criteria: "Inclusion criteria required patients $\geq 18$ years of age with histologically or cytologically confirmed malignant disease who were chemotherapy naive and scheduled to receive HEC as defined by the NCCN guidelines."

Exclusion criteria: "Patients were excluded if they had documented nausea or vomiting in the 24 hours prior to beginning antiemetic therapy (Day -2). Other Inclusion criteria included serum creatinine $\geq 2 \mathrm{mg} / \mathrm{dL}$; serum bilirubin $\geq 2 \mathrm{mg} / \mathrm{dL}$; SGOT or SGPT $\geq 3$ times the upper limit of normal, and absolute neutrophil count $\leq 1500 \mathrm{~mm} 3$. Participants of childbearing age (male and female) must have consented to use of adequate contraception throughout protocol therapy; women of childbearing age must have had a negative serum pregnancy test; no severe cognitive compromise; no history of CNS disease (e.g. uncontrolled brain metastases, seizure disorder); no treatment with another antipsychotic agent such as risperidone (Risperdal), quetiapine (Seroquel), clozapine (Clozaril), phenothiazine, or butyrophenone for 30 day prior to or during protocol therapy. No chronic phenothiazine administration as an antipsychotic agent was allowed, but participants may have been receiving prochlorperazine (Compazine) and other phenothiazines as rescue antiemetic therapy. No concurrent use of amifostine 
(Ethyol); no concurrent abdominal radiotherapy; no concurrent use of quinolone antibiotic therapy; no chronic alcoholism (as determined by the investigator); no known hypersensitivity to olanzapine; no known cardiac arrhythmia, uncontrolled CHF, or acute MI within the previous 6 months and no history of uncontrolled diabetes, no history of stroke, and no dementia related psychoses."

Baseline characteristics: cancer type: breast; lung; Hodgkins; head and neck; sarcoma, $63 \%$ of the participants were women. In the olanzapine group 56\% were women compared to $70 \%$ in the aprepitant group. Median age was 54 in the olanzapine group compared to 60 in the aprepitant group

Setting and location: San Antonia Military Medical Centre, USA

Number of people screened: 20

Number of participants randomised - all: 20

Number randomised to each group: olanzapine: 10; aprepitant: 10

Number receiving treatment as allocated: olanzapine: 10; aprepitant: 10

Number not receiving treatment as allocated: olanzapine: 0; aprepitant: 0

Number dropped out: olanzapine: 1; aprepitant: 0 (no follow-up data for any outcome available)

Number excluded from analysis (for all outcomes): olanzapine: 1 aprepitant: 0

- reason 1 - lost diary

Number completed: olanzapine: 9; aprepitant: 9

Interventions

Intervention group $\mathbf{A}(\mathrm{N}=10)$ : day 2-4: olanzapine $10 \mathrm{mg}$, oral, once/day

Intervention group $\mathbf{B}(\mathrm{N}=10)$ : day - 1: placebo, oral, once/day

day 1-3: aprepitant $125 \mathrm{mg}$, oral, once/day

day 4: placebo, oral, once/day

Use of additional treatments if any (co-interventions, additional analgesia):

day 1: dexamethasone $12 \mathrm{mg} \mathrm{IV}$, palonosetron $0.25 \mathrm{mg} \mathrm{IV}$

day 2-5: dexamethasone $4 \mathrm{mg}$ oral

"Breakthrough or rescue medicines were prescribed by the treating physician at their discretion" (N.B. olanzapine or placebo was given for the 2 days prior to giving chemo)

\section{Outcomes}

Primary outcomes

- Proportion of participants with complete response, defined as no emesis and no use of rescue medicines - acute period (day 1), the delayed period (day 2-4), and the total period (day 1-4)

- Complete response rates for the anticipatory period (day 2, day 1 for the second cycle of chemotherapy only)

Secondary outcomes

- Nausea was recorded as present if participants answered $\geq 1$ for question 3 on the MDASI

- Significant nausea was recorded as present if participants answered $\geq 3$ on the MDASI

Funding No information

Declarations of Interest

"All of the other authors have no conflicts of interest relevant to the contents of this manuscript."

\section{Notes}

\section{Risk of bias}

Bias Authors' judgement Support for judgement


Shumway 2015 (Continued)

Random sequence genera- Low risk tion (selection bias)
Quote: "Randomization was computer generated and performed by the central oncology pharmacy."

Comment: good description of how the randomisation sequence was generated

\begin{tabular}{ll}
\hline $\begin{array}{l}\text { Allocation concealment } \\
\text { (selection bias) }\end{array}$ & Quote: "Randomization was computer generated and performed by the cen- \\
& tral oncology pharmacy." \\
& Comment: it is assumed that the participants were allocated to groups by the \\
central oncology pharmacy and so the treating physicians were not aware of \\
the treatment group
\end{tabular}

\begin{tabular}{|c|c|c|}
\hline $\begin{array}{l}\text { Blinding of participants } \\
\text { and personnel (perfor- } \\
\text { mance bias) } \\
\text { All outcomes }\end{array}$ & Low risk & $\begin{array}{l}\text { Quote: "The standard (aprepitant) arm received placebo for Day - } 2 \text { and Day - } \\
1 \text { prior to each cycle of chemotherapy given as a black gel capsule prepared by } \\
\text { the central oncology pharmacy." } \\
\text { Comment: attempts were made to maintain blinding through the use of place- } \\
\text { bo capsules which were identical to the active treatment and the comparison } \\
\text { treatment }\end{array}$ \\
\hline
\end{tabular}

Blinding of outcome as- Low risk sessment (detection bias) All outcomes
Quote: "Patients were given a daily diary to record episodes of emesis/retching and use of rescue medicines. In addition, patients were asked to fill out the MD Anderson Symptom Inventory (MDASI)."

"Daily queries from the investigators were performed to remind patients to fill out forms and ask about toxicities."

Comment: as the outcomes were primarily participant-reported and the participants were blinded to treatment, it can be assumed that there was adequate blinding of outcome assessment

Only one participant was lost to follow-up from either arm $(1 / 20=5 \%)$. The reason for the participant not being analysed was given

$\begin{array}{ll}\begin{array}{l}\text { Incomplete outcome data } \\ \text { (attrition bias) }\end{array} & \text { Low risk } \\ \text { All outcomes } & \begin{array}{l}\text { Only one participant was lost to follow-up from either arm }(1 / 20=5 \%) . \text { The } \\ \text { reason for the participant not being analysed was given }\end{array}\end{array}$

All outcomes

No protocol available on ClinicalTrials.gov

Selective reporting (re- Unclear risk $\quad$ No protocol available on ClinicalTrials.gov
porting bias)

\begin{tabular}{lll}
\hline $\begin{array}{l}\text { Other bias (validation of } \\
\text { instruments) }\end{array}$ & Low risk & Main outcomes were dichotomous. MDASI scale used for AEs \\
\hline Other bias & High risk & 10 participants per treatment group \\
\hline
\end{tabular}

\section{Wang 2015}

\begin{tabular}{ll} 
Methods & Trial method: RCT \\
& Randomised: yes \\
& Blinding: nil stated, likely unblinded \\
Arms: 2 & \\
& MEC or HEC: HEC \\
& Multicentre/single-centre: 1 \\
& Dates: February 2010 - June 2012 \\
\hline
\end{tabular}

Participants $\quad \mathrm{N}=84$ (42 per arm)

Age: $39-76$ years

Gender: Male + Female 
Wang 2015 (Continued)

Inclusion criteria: pathologically and/or cytologically confirmed NSCLC, in preoperative/postopera-

tive/or inoperable phase of illness, receiving cisplatin-gemcitabine

Exclusion criteria: brain metastases and gastrointestinal obstruction excluded

Participant (baseline) characteristics: main diagnosis NSCLC (squamous cell or adenocarcinoma)

Setting and location: hospital, China

Number of people screened: 84

Number of participants randomised - all: 84

Number randomised to each group: 42

Number receiving treatment as allocated: 84

Number not receiving treatment as allocated: nil reported

Number dropped out: nil reported (no follow-up data for any outcome available)

Number excluded from analysis (for all outcomes): nil reported

Number completed: 84

Interventions Intervention group A ( $=42)$ : olanzapine $10 \mathrm{mg}$ oral daily for 8 days with ondansetron $8 \mathrm{mg}$ IV pre-

chemotherapy

Control group B ( $\mathrm{N}=42)$ : ondansetron $8 \mathrm{mg}$ IV pre-chemotherapy

Use of additional treatments if any (co-interventions, additional analgesia): nil

\begin{tabular}{|c|c|c|}
\hline Outcomes & \multicolumn{2}{|c|}{$\begin{array}{l}\text { Outcomes of interest in the review } \\
\text { Primary outcomes } \\
\text { - Number of participants with no nausea and no vomiting at } 24 \text { and } 48 \text { hours (Grade } 0 \text { CINV) } \\
\text { Secondary outcomes } \\
\text { - } 1 \text { AEs } \\
\text { Other outcomes reported by the study: } \\
\text { Severity of CINV on WHO anticancer drug toxicity grading criteria } 1998 \text { (unvalidated score) }\end{array}$} \\
\hline Funding & No information & \\
\hline Declarations of Interest & No information & \\
\hline Notes & & \\
\hline Risk of bias & & \\
\hline Bias & Authors' judgement & Support for judgement \\
\hline $\begin{array}{l}\text { Random sequence genera- } \\
\text { tion (selection bias) }\end{array}$ & Unclear risk & $\begin{array}{l}\text { Quote: "These } 84 \text { patients were equally randomized into intervention group } \\
\text { and control group." } \\
\text { Comment: method of randomisation was not described }\end{array}$ \\
\hline $\begin{array}{l}\text { Allocation concealment } \\
\text { (selection bias) }\end{array}$ & Unclear risk & $\begin{array}{l}\text { Quote: "These } 84 \text { patients were equally randomized into intervention group } \\
\text { and control group." } \\
\text { Comment: method of allocation was not described }\end{array}$ \\
\hline $\begin{array}{l}\text { Blinding of participants } \\
\text { and personnel (perfor- } \\
\text { mance bias) } \\
\text { All outcomes }\end{array}$ & High risk & $\begin{array}{l}\text { Quote: "The control group was intravenously administered with ondansetron } \\
8 \text { mg } 30 \text { minutes before chemotherapy. In the intervention group, however, } \\
\text { the patients were intravenously administered with ondansetron } 8 \mathrm{mg} 30 \mathrm{~min} \text { - } \\
\text { utes before chemotherapy, then olanzapine } 10 \mathrm{mg} \text { was orally administered for } \\
8 \text { days, beginning from the first morning of chemotherapy." } \\
\text { Comment: no blinding described and intervention group received tablets } \\
\text { when the control group did not }\end{array}$ \\
\hline
\end{tabular}


Wang 2015 (Continued)

Blinding of outcome as- Unclear risk $\quad$ Quote: "The CINV was evaluated after one chemotherapy cycle." sessment (detection bias) Comment: method of outcome assessment was not described All outcomes

\begin{tabular}{lll}
$\begin{array}{l}\text { Incomplete outcome data } \\
\text { (attrition bias) } \\
\text { All outcomes }\end{array}$ & Low risk & $\begin{array}{l}\text { Comment: results are reported for all } 84 \text { participants who were randomised. } \\
\text { No CONSORT diagram is available. }\end{array}$ \\
\hline $\begin{array}{l}\text { Selective reporting (re- } \\
\text { porting bias) }\end{array}$ & Unclear risk & $\begin{array}{l}\text { Comment: no protocol available on www.clinicaltrials.gov or Chinese registry } \\
\text { of clinical trials (www.chictr.org.cn) }\end{array}$
\end{tabular}

\begin{tabular}{|c|c|c|}
\hline $\begin{array}{l}\text { Other bias (validation of } \\
\text { instruments) }\end{array}$ & High risk & $\begin{array}{l}\text { Comment: WHO anticancer drug toxicity grading criteria (1998), an unvalidat- } \\
\text { ed scale was used, therefore we only incorporated dichotomous data from the } \\
\text { paper in the review }\end{array}$ \\
\hline
\end{tabular}

\begin{tabular}{ll}
\hline Other bias $\quad$ High risk $\quad$ High risk of bias based on number of participants per arm $(\mathrm{N}=42)$ \\
\hline
\end{tabular}

\section{Zhang 2017}

\begin{tabular}{|c|c|}
\hline Methods & $\begin{array}{l}\text { Trial method: RCT, parallel-group } \\
\text { MEC or HEC: MEC and HEC } \\
\text { Randomised: yes } \\
\text { Dates: not stated }\end{array}$ \\
\hline Participants & $\begin{array}{l}\mathrm{N}=120 \text { ( } 60 \text { per arm) } \\
\text { Age: } \geq 18 \text { years, } 54.18 \pm 10.24 \\
\text { Gender: Female M male } \\
\text { Inclusion criteria: histologically or cytologically confirmed malignant disease. Accept chemotherapy } \\
\text { for the first time } \\
\text { Patients who will receive HEC (cisplatin } \geq 70 \mathrm{mg} / \mathrm{m} 2 \text {, adriamycin in combination with cyclophos- } \\
\text { phamide, cyclophosphamide } \geq 1500 \mathrm{mg} / \mathrm{m} 2 \text {, adriamycin }>60 \mathrm{mg} / \mathrm{m} 2 \text {, epirubicin }>90 \mathrm{mg} / \mathrm{m} 2, \text { dacar- } \\
\text { bazine, ifosfamide } \geq 2 \mathrm{~g} / \mathrm{m} 2 \text { ) or MEC (carboplatin } \geq 300 \mathrm{mg} / \mathrm{m} 2, \mathrm{cyclophosphamide} \geq 600-1000 \mathrm{mg} / \mathrm{m} 2 \text {, } \\
\text { adriamycin }>50 \mathrm{mg} / \mathrm{m} 2 \text { ). } \\
\text { Exclusion criteria: pregnant or breast-feeding. Uncontrolled psychosis history. Inability or unwilling- } \\
\text { ness to understand or co-operate with study procedures. CNS tumours primary or secondary. Concur- } \\
\text { rent abdominal radiotherapy } \\
\text { History of uncontrolled diabetes mellitus. Patients of prostatic hyperplasia, paralytic ileus, narrow } \\
\text { feet glaucoma. Known cardiac arrhythmia, uncontrolled CHF, or acute MI within the previous } 6 \text { months. } \\
\text { Pre-existing nausea or vomiting. Inadequate haematological function and abnormal liver and renal } \\
\text { function. History of sensitivity to olanzapine. Concurrent application of quinolone antibiotic therapy. } \\
\text { Treatment with another antipsychotic agent such as risperidone,quetiapine, clozapine, phenothiazine, } \\
\text { or butyrophenone for } 30 \text { days prior to or during the chemotherapy. Cytochrome P450 } 3 \text { A4 substrates } \\
\text { within } 7 \text { days (terfenadine, cisapride, astemizole, pimozide). Concurrent application of systemic corti- } \\
\text { costeroids. Active infection or gastrointestinal dysfunction } \\
\text { Baseline characteristics: } \\
\text { Setting and location: First Affiliated Hospital of Harbin Medical University, Harbin, Heilongjiang, China, } \\
150000 \\
\text { Number of people screened: not reported } \\
\text { Number of participants randomised - all: } 120 \\
\text { Number randomised to each group: olanzapine: } 60 \text {; control: } 60 \\
\text { Number receiving treatment as allocated: olanzapine: } 60 ; \text { control: } 60\end{array}$ \\
\hline
\end{tabular}

Interventions

Intervention group A ( $\mathrm{N}=60)$ : day 1-4: olanzapine, $5 \mathrm{mg}$, orally, twice/day orally

Control group $B(N=60)$ : no additional treatment 
Use of additional treatments if any (co-interventions, additional analgesia):

day 1: aprepitant: $125 \mathrm{mg}$ capsule orally, palonosetron: $0.25 \mathrm{mg}$ IV dexamethasone: $6 \mathrm{mg}$ IV; 30-60 minutes before chemotherapy

day 2-3: aprepitant $80 \mathrm{mg}$ capsule daily in the morning and dexamethasone $3.75 \mathrm{mg}$ IV

day 4: dexamethasone $3.75 \mathrm{mg}$ IV

Primary outcomes
- Proportion of participants receiving HEC/MEC with complete response in overall phase
- SAEs
Secondary outcomes
- Proportion of participants receiving $\mathrm{HEC} / \mathrm{MEC}$ with complete response in the acute phase
- Proportion of participants receiving $\mathrm{HEC} / \mathrm{MEC}$ with complete response in the delayed phase
- Proportion of participants receiving $\mathrm{HEC} / \mathrm{MEC}$ with no vomiting in the overall phase
- Proportion of participants receiving $\mathrm{HEC} / \mathrm{MEC}$ with no vomiting in the acute phase
- Proportion of participants receiving $\mathrm{HEC} / \mathrm{MEC}$ with no vomiting in the delayed phase
- Other AEs

Funding

First Affiliated Hospital of Harbin Medical University Harbin Medical University

\section{Declarations of Interest}

"All Principal Investigators ARE employed by the organization sponsoring the study."

\section{Notes}

\section{Risk of bias}

Bias Authors' judgement Support for judgement

Random sequence genera- Unclear risk tion (selection bias)

Quote: "Eligible patients will be randomized to receive different antiemetic regimens."

Comment: states that study is randomised by no information on the clinical trials website of methods for generation of randomisation sequence used. In addition, the results are reported separating out those who had HEC and MEC. However, it is not clear if these groups were stratified at randomisation or if they represent a post-hoc subgroup

\begin{tabular}{lll}
\hline $\begin{array}{l}\text { Allocation concealment } \\
\text { (selection bias) }\end{array}$ & Unclear risk & $\begin{array}{l}\text { Comment: method of allocation was not described. No information regarding } \\
\text { allocation concealment is given on the clinical trials web site }\end{array}$
\end{tabular}

\begin{tabular}{|c|c|c|}
\hline $\begin{array}{l}\text { Blinding of participants } \\
\text { and personnel (perfor- } \\
\text { mance bias) } \\
\text { All outcomes }\end{array}$ & High risk & $\begin{array}{l}\text { Comment: the clinical trials website indicates that the trial was 'open' and so } \\
\text { participants and healthcare professionals would have been aware of the treat- } \\
\text { ment group. }\end{array}$ \\
\hline $\begin{array}{l}\text { Blinding of outcome as- } \\
\text { sessment (detection bias) } \\
\text { All outcomes }\end{array}$ & High risk & $\begin{array}{l}\text { Quote: "During the treatment, any grade of nausea and vomiting should be } \\
\text { recorded in order to evaluate the complete response rate of CINV,nausea pa- } \\
\text { tients will be measured by a visual analogue scale (VAS), other AEs should be } \\
\text { recorded as well." } \\
\text { Comment: no information on whether participant self-reported or if asses- } \\
\text { sor reported, however in the context of both unblinded participants and un- } \\
\text { blinded assessors either represents a high risk of bias. The clinical trials web- } \\
\text { site indicates that the trial was 'open' and so outcome assessors (mainly par- } \\
\text { ticipants) would have been aware of the treatment group. }\end{array}$ \\
\hline
\end{tabular}


Zhang 2017 (Continued)

Incomplete outcome data Low risk (attrition bias)

All outcomes
Comment: $6 / 120(5 \%)$ participants did not complete the study. The number of participants is not significantly different between the two groups $(6.7 \% \mathrm{vs}$ $3.3 \%$ ). Basic reasons are provided and are not different between the groups.
Selective reporting (re- Unclear risk porting bias)
Comment: the clinical trial protocol is available at ClinicalTrials.gov (Protocol 02484911). Some of the results are posted here. It is clear from the protocol that the outcomes changed during the trial process. Some of the original outcomes that were planned to be reported initially were not reported (e.g. QOL). In addition, the initial plan was not to analyse HEC and MEC populations separately and this change appeared to be made after the trial had started.

Other bias (validation of Low risk Validated VAS scale. Only dichotomous data are presented
instruments)

instruments)

Other bias Unclear risk $\quad 60$ per arm

Zhao 2014

$\begin{array}{ll}\text { Methods } & \text { Trial method: RCT } \\ & \text { Randomised: yes } \\ & \text { MEC or HEC: MEC and HEC } \\ \text { Blinding: nil } \\ \text { Arms: } 2 \\ \text { Multicentre/single-centre: single } \\ \text { Dates: not stated }\end{array}$

\section{Participants}

$\mathrm{N}=80$ (40 per arm)

Age: average age was $55.9 \pm 8.27$; control group: average age was $56.8 \pm 9.23$

Gender: Male + Female

Inclusion criteria: patients with HEC/MEC drugs. All patients diagnosed as malignant tumours with cytology and pathology; ECOG physical stamina score $\geq 2$, the count of neutrophil count $\geq 1.5^{\star} 109 / \mathrm{L}, \mathrm{Hb} \geq$ $100 \mathrm{~g} / \mathrm{L}$, platelet $\geq 80^{\star} 109 / \mathrm{L}$, serum creatinine $\leq 45 \mu \mathrm{mol} / \mathrm{L}$, serum bilirubin $<20 \mu \mathrm{mol} / \mathrm{L}$; normal cardiac function according to ultrasonic; without cognitive impairment

Exclusion criteria: concurrent chemoradiotherapy; partial or complete intestinal obstruction;Vestibular dysfunction; brain metastasis; electrolyte disturbances; uremia; continuous application of opioid drugs; gastric dynamic disorders; physical factors such as anxiety

\section{Baseline characteristics:}

Main diagnosis: intervention group: lung cancer 17 people; colorectal cancer 11 people; breast cancer 12 people. Control group: lung cancer 16 people; colorectal cancer 13 people; breast cancer 11 people Other important effect modifiers, if applicable (e.g. radiotherapy): radiotherapy excluded

Setting and location: hospital, China

Number of people screened: 80

Number of participants randomised - all: 80

Number randomised to each group: 40

Number receiving treatment as allocated: all 
Zhao 2014 (Continued)

Number not receiving treatment as allocated: nil

Number dropped out: nil

Number excluded from analysis (for all outcomes): nil

Number completed: 80

Interventions

Intervention group $\mathbf{A}(\mathrm{N}=40)$ : day 1-end of chemotherapy: diphenhydramine $(20 \mathrm{mg} \mathrm{IM})+$ on-

dansetron (16 mg IV) + dexamethasone (10 mg IV) + olanzapine $5 \mathrm{mg}$ oral at night

Control group B $(\mathrm{N}=40)$ : day 1-end of chemotherapy: diphenhydramine (20 mg IM) + ondansetron (16

$\mathrm{mg}$ IV) + dexamethasone (10 mg IV)

Use of additional treatments if any: no information

\begin{tabular}{ll}
\hline Outcomes & Primary outcomes \\
& - Efficacy \\
& Secondary outcomes \\
& - AEs \\
\hline Funding & No information \\
\hline Declarations of Interest & No information \\
\hline Notes &
\end{tabular}

\section{Risk of bias}

\begin{tabular}{lll}
\hline Bias & Authors' judgement & Support for judgement \\
\hline $\begin{array}{l}\text { Random sequence genera- } \\
\text { tion (selection bias) }\end{array}$ & Unclear risk & $\begin{array}{l}\text { Quote: "patients were randomly divided into two groups..." } \\
\text { Comment: method of randomisation not described }\end{array}$ \\
\hline $\begin{array}{l}\text { Allocation concealment } \\
\text { (selection bias) }\end{array}$ & Unclear risk & $\begin{array}{l}\text { Quote: "patients were randomly divided into two groups..." } \\
\text { Comment: allocation concealment not described }\end{array}$ \\
\hline $\begin{array}{l}\text { Blinding of participants } \\
\text { and personnel (perfor- } \\
\text { mance bias) } \\
\text { All outcomes }\end{array}$ & High risk & $\begin{array}{l}\text { Comment: no record of blinding of participants or assessors, but it is likely that } \\
\text { participants and personnel were not blinded though it was not described }\end{array}$ \\
\hline
\end{tabular}

\begin{tabular}{ll}
\hline Blinding of outcome as- & Unclear risk
\end{tabular}

\begin{tabular}{lll}
\hline $\begin{array}{l}\text { Incomplete outcome data } \\
\text { (attrition bias) } \\
\text { All outcomes }\end{array}$ & Low risk & Quote: "All patients were successfully completed treatment..." \\
\hline $\begin{array}{l}\text { Selective reporting (re- } \\
\text { porting bias) }\end{array}$ & Unclear risk & Comment: no protocol available on clinicaltrials.gov \\
\hline $\begin{array}{l}\text { Other bias (validation of } \\
\text { instruments) }\end{array}$ & High risk & $\begin{array}{l}\text { WHO digestive tract reaction classification standard of anticancer drugs, an } \\
\text { unvalidated scale }\end{array}$ \\
\hline Other bias & High risk & 40 per treatment arm \\
\hline
\end{tabular}


AE: adverse event; CCF: congestive cardiac failure; CHF: congestive heart failure; CINV: chemotherapy-induced nausea and vomiting; CNS: central nervous system; CTCAE: Common Toxicity Criteria for Adverse Events; ECOG: European Cooperative Oncology Group; EORTC QLQC30: European Organisation for Research and Treatment of Cancer quality of life questionnaire; FACT-G: Functional Assessment of Cancer Therapy - General version 3; FLIE: Functional Living Index-Emesis; HADS: Hospital Anxiety and Depresssion Scale; Hb: haemoglobin; HEC: highly emetogenic chemotherapy; HSCT: hematopoietic stem cell transplantation; IM: intramuscular; IV: intravenous; MEC: moderately emetogenic chemotherapy; MASCC MAT: Multinational Association for Supportive Care in Cancer Antiemetic Tool; MDASI: MD Anderson Symptom Inventory; MI: myocardial infarction; NSCLC: non-small-cell lung carcinoma; P.O.: per oras (meaning "by mouth"); QOL: quality of life; RCT: randomised controlled trial; SAE: serious adverse event; SGOT: serum glutamic oxaloacetic transaminase; SGPT: serum glutamic pyruvic transaminase; VAS: visual analogue scale; WHO: World Health Organization; WHO-QOL-BREF: World Health Organization Quality of Life-BREF

\section{Characteristics of excluded studies [ordered by study ID]}

\begin{tabular}{ll}
\hline Study & Reason for exclusion \\
\hline Babu 2016 & $\begin{array}{l}\text { RCT studying olanzapine as an antiemetic in adults with cancer. Excluded due to intervention. } \\
\text { Different doses and regimen of dexamethasone administration in olanzapine arm compared to } \\
\text { aprepitant arm, leading us to be unable to attribute study results with certainty to the sole action } \\
\text { of olanzapine }\end{array}$ \\
\hline
\end{tabular}

EUCTR2015-002294-38-DK W Wrong study design. Non-randomised, single-arm, open-label study

\begin{tabular}{ll}
\hline Flank 2015a & Wrong patient population (paediatric) \\
\hline Flank 2015b & Wrong patient population (paediatric) \\
\hline Fountaine 2010 & Wrong patient population (paediatric) \\
\hline
\end{tabular}

\begin{tabular}{|c|c|}
\hline Guntsch 2012 & Early termination 'poor enrolment' \\
\hline Hashimoto 2016 & Wrong intervention, dose comparison trial, $10 \mathrm{mg}$ versus $5 \mathrm{mg}$, only brief abstract available \\
\hline ISRCTN58624349 & Terminated due to poor enrolment \\
\hline Kwatra 2013 & $\begin{array}{l}\text { Abstract only, information received from study author, who confirmed nausea and vomiting not an } \\
\text { outcome. Wrong indication }\end{array}$ \\
\hline Long 2017 & Wrong patient population (paediatric),$<10$ participants per arm \\
\hline Mukhopadhyay 2012 & $\begin{array}{l}\text { Abstract only, information received from study author, who confirmed nausea and vomiting not an } \\
\text { outcome }\end{array}$ \\
\hline Nakashima 2015 & Wrong study design, trial protocol \\
\hline Navari 2009a & $\begin{array}{l}\text { RCT studying olanzapine as an antiemetic in adults with cancer. Excluded due to intervention. Dif- } \\
\text { ferent doses and regimen of dexamethasone administration in olanzapine arm versus comparator } \\
\text { arm, leading us to be unable to attribute study results with certainty to the sole action of olanzap- } \\
\text { ine }\end{array}$ \\
\hline Navari 2010a & $\begin{array}{l}\text { RCT studying olanzapine as an antiemetic in adults with cancer. Excluded due to intervention. Dif- } \\
\text { ferent doses and regimen of dexamethasone administration in olanzapine arm versus comparator } \\
\text { arm, leading us to be unable to attribute study results with certainty to the sole action of olanzap- } \\
\text { ine }\end{array}$ \\
\hline Navari 2011 & $\begin{array}{l}\text { RCT studying olanzapine as an antiemetic in adults with cancer. Excluded due to intervention. Dif- } \\
\text { ferent doses and regimen of dexamethasone administration in olanzapine arm versus comparator } \\
\text { arm, leading us to be unable to attribute study results with certainty to the sole action of olanzap- } \\
\text { ine }\end{array}$ \\
\hline
\end{tabular}




\begin{tabular}{ll}
\hline Study & Reason for exclusion \\
\hline Navari 2016b & $\begin{array}{l}\text { RCT studying olanzapine as an antiemetic in adults with cancer. Excluded due to intervention. Dif- } \\
\text { ferent doses and regimen of dexamethasone administration in olanzapine arm versus comparator } \\
\text { arm, leading us to be unable to attribute study results with certainty to the sole action of olanzap- } \\
\text { ine }\end{array}$ \\
\hline NCT00124930 & Early termination 'due to low enrolment' \\
\hline NCT01148264 & Terminated, no results, no contact details \\
\hline Slimano 2016 & $\begin{array}{l}\text { Wrong study design. Abstract only, information received from study author, who confirmed } 2 \text { co- } \\
\text { horts, not an RCT }\end{array}$ \\
\hline Yanai 2015 & Wrong intervention, dose comparison trial, 10 mg vs 5 mg, trial completed, no results available \\
\hline
\end{tabular}

mg: milligram;RCT: randomised controlled trial; vs: versus

Characteristics of studies awaiting assessment [ordered by study ID]

Chasick 2012

\begin{tabular}{|c|c|}
\hline Methods & $\begin{array}{l}\text { Trial method: RCT } \\
\text { MEC or + HEC: MEC or HEC } \\
\text { Randomised: yes } \\
\text { Blinding: double-blind } \\
\text { Arms: } 2 \\
\text { Multicentre/single-centre: single } \\
\text { Dates: not stated }\end{array}$ \\
\hline Participants & Not stated \\
\hline Interventions & $\begin{array}{l}\text { Intervention group A ( } \mathrm{N}=\text { ?): day } 1 \text { olanzapine } 10 \mathrm{mg} \text { oral (blinded), dexamethasone } 12 \mathrm{mg} \text { oral, } \\
\text { ondansetron } 16 \mathrm{mg} \text { oral; day 2-3 olanzapine } 10 \mathrm{mg} \text { oral daily, dexamethasone } 4 \mathrm{mg} \text { oral, twice/day } \\
\text { Intervention group B ( } \mathrm{N}=\text { ?): day } 1 \text { aprepitant } 125 \mathrm{mg} \text { oral (blinded), dexamethasone } 12 \mathrm{mg} \text { oral, } \\
\text { ondansetron } 16 \mathrm{mg} \text { oral; day 2-3 aprepitant } 80 \mathrm{mg} \text { oral daily, dexamethasone } 4 \mathrm{mg} \text { oral twice/day } \\
\text { Use of additional treatments if any (co-interventions, additional analgesia): Rescue therapy for } \\
\text { both arms - prochlorperazine } 10 \mathrm{mg} \text { oral every } 4-6 \text { hours as needed }\end{array}$ \\
\hline Outcomes & $\begin{array}{l}\text { Primary outcomes } \\
\text { - Nausea score using a 10-cm NVAS } \\
\text { Secondary outcomes } \\
\text { - Complete response in the acute period } \\
\text { - Complete response in the delayed period }\end{array}$ \\
\hline Notes & $\begin{array}{l}\text { Funding: no information } \\
\text { Declaration of interest: no information } \\
\text { Conference abstract only. Awaiting further information from study authors }\end{array}$ \\
\hline
\end{tabular}




\section{CTRCC-14004093}

\section{Methods}

Trial method: randomised cross-over control

MEC or HEC: not stated

Randomised: yes

Blinding: not stated

Arms: not stated

Multicentre/single-centre: not stated

Dates: January 2014 - June 2016

\begin{abstract}
Participants $\quad \mathrm{N}=320$
Age: $18-70$ years

Gender: Male + Female

Inclusion criteria: malignant tumour patients can be treated with chemotherapy, disease is unlimited; chemotherapy drugs including cisplatin in $60 \mathrm{mg} / \mathrm{m} 2$. Sequential two cycles of chemotherapy drugs, dose, dosing sequence and dosing method must be completely consistent; patients on chemotherapy cycle don't accept any other chemotherapy drugs during day 2-4, don't accept experimental design other antinausea, composed, psychotropic drugs and hormonal therapy during day 1-5; patients' ages from 18-75 years, any gender, Karnoffsky Performance Score $\geq 60$, is expected to survive > 3 months; blood picture, liver and kidney function, ECG basic normal, comply with the indications of chemotherapy; it should be > 2 weeks after the last chemotherapy; participants should sign the informed consent

Exclusion criteria: nursing and pregnant women; digestive tract obstruction; severe heart disease, kidney disease; people with epilepsy or the use of psychotropic drugs and narcotics; people used antinausea drugs or within 24 hours before chemotherapy; people have brain metastasis and intracranial pressure caused by vomiting, or influence the vomiting; allergic to olanzapine; chemotherapy contraindications; people is in 3 months or other clinical subjects.
\end{abstract}

\section{Baseline characteristics:}

Setting and location: not stated

Number of people screened: not stated

Number of participants randomised: all

Number randomised to each group: not stated

Number receiving treatment as allocated: not stated

Number not receiving treatment as allocated: not stated
Interventions
Intervention group $\mathbf{A}(\mathrm{N}=$ ?): olanzapine

Intervention group $\mathbf{B}(\mathrm{N}=$ ? $)$ : not stated

Control group $\mathrm{C}(\mathrm{N}=$ ?): not stated

Use of additional treatments if any (co-interventions, additional analgesia): not stated

\begin{tabular}{ll} 
Outcomes & Primary outcomes \\
- Not stated \\
Secondary outcomes \\
- Not stated \\
\hline Notes & $\begin{array}{l}\text { Completed study on unpublished trials database. No published or unpublished results. Awaiting } \\
\text { further information from study authors }\end{array}$ \\
\hline
\end{tabular}


Jeon 2017 (Continued)

\section{Arms: 2}

Multicentre/single-centre: not stated

Dates: not stated

Participants $\quad$ Age: not stated
Gender: not stated
Inclusion criteria: not stated
Exclusion criteria: not stated
Baseline characteristics: not stated
Setting and location: not stated
Number of people screened: not stated
Number of participants randomised - all: 56
Number randomised to each group: not stated
Number receiving treatment as allocated: not stated
Number not receiving treatment as allocated: not stated
Number dropped out: 2 (no follow-up data for any outcome available)
Number excluded from analysis (for all outcomes): not stated
Number completed: 54 (29 in olanzapine arm and 25 in placebo arm)

Interventions

Intervention group $\mathbf{A}(\mathrm{N}=$ ?): $10 \mathrm{mg}$ of olanzapine orally

Control group $\mathrm{C}(\mathrm{N}=$ ?): matching placebo daily on day 1 to 4

Use of additional treatments if any (co-interventions, additional analgesia): palonosetron and dexamethasone

\section{Outcomes}

Primary outcomes

- Complete response (no emesis and no use of rescue medication) for the acute phase (0 - 24 hours after chemotherapy)

Secondary outcomes

- 1. Complete responses for the delayed (24-120 hours)

- 2. Complete responses for overall phase (0-120 hours)

- 3. Proportion of significant emesis (VAS $>=25 \mathrm{~mm}$ ) for overall phase

- 4. Use of rescue medications

Others: effect on QOL by Functional Living Index-Emesis (FLIE) questionnaire

$\begin{array}{ll}\text { Notes } & \text { Funding: no information } \\ \text { Declaration of interest: no information } \\ \text { Conference abstract only. Awaiting further information from study authors }\end{array}$

Mao 2011

Methods Trial method: randomised cross-over study

MEC or HEC: not stated

Randomised: yes

Blinding: no description given

Arms: 2

Multicentre/single-centre: single centre

Dates: June 2008-December 2009

$\begin{array}{ll}\text { Participants } & \mathrm{N}=46 \\ & \text { Age: } 38-78 \text { (average } 52.6 \text { years) }\end{array}$


Mao 2011 (Continued)

Gender: Male + Female

Inclusion criteria:

"All patients are diagnosed and confirmed via ......" and "All participants must be able to tolerate at least 2 weeks or more of chemotherapy"

Lung cancer; Oesophageal cancer; Stomach cancer/colorectal; Breast cancer; Ovarian cancer; Multiple myeloma; Nasaopharyngeal cancer

Some treated with radiotherapy

Exclusion criteria: not explicitly stated

Baseline characteristics:

"20 had lung cancer, 14 with oesophageal, 3 stomach, 3 with breast and 2 with colorectal cancer, 2 with ovarian cancer and 1 with multiple myeloma, 1 with nasopharyngeal cancer."

Setting and location: not stated

Not explicitly described

Number of people screened: not described

Number of participants randomised - all: 46

Number randomised to each group: 46 (number in group A and B is not described; the paper regrouped them in analysis as Intervention/Control)

Number receiving treatment as allocated: 46

Number not receiving treatment as allocated: 0 ("All participants completed 2 weeks of

chemotherapy")

Number dropped out: 0

Number excluded from analysis (for all outcomes): 0

Number completed: 46

Interventions

Intervention group A + B; olanzapine + granisetron \& hexadecrol $(\mathrm{N}=46)$ :

Participants sorted into the 2 groups $A$ and $B$, and regrouped into Intervention/Control for analysis A: 1st week of chemotherapy, granisetron $(3 \mathrm{mg} / 50 \mathrm{~mL})$ and Hexadecrol 5 - $10 \mathrm{mg}$ via IV route ; and olanzapine ( $5 \mathrm{mg}$ per tablet) $5 \mathrm{mg}$ twice/d; given pre- and postchemotherapy 2nd-week of chemotherapy, granisetron $(3 \mathrm{mg} / 50 \mathrm{~mL}$ ) and hexadecrol 5-10 $\mathrm{mg}$ IV

B: 1st week of chemotherapy, granisetron $(3 \mathrm{mg} / 50 \mathrm{~mL})$ and hexadecrol 5-10 $\mathrm{mg}$ given via IV route; no olanzapine given; given pre- and postchemotherapy

2nd week of chemotherapy granisetron $(3 \mathrm{mg} / 50 \mathrm{~mL}$ ) and hexadecrol 5-10 $\mathrm{mg}$ IV + olanzapine $5 \mathrm{mg}$ twice/day

Control group: granisetron \& hexadecrol without olanzapine $(\mathrm{N}=46)$ : participants sorted into the 2 groups $\mathrm{A}$ and $\mathrm{B}$, and regrouped into Intervention/control for analysis

Use of additional treatments if any (co-interventions, additional analgesia): none

Outcomes

Primary outcomes

- Effectiveness of treatment for up to 7 day post-chemo measurement using:

* WHO Anti-Cancer Acute/Non-Acute Gastrointestinal Adverse Reaction Grading,

$\square 0$ - no nausea/vomiting

$\square$ I - nausea with no vomiting

$\square$ II - daily vomiting 1-2 times, no affecting normal oral intake and life

$\square$ III - daily vomiting 3-5 times, affecting oral intake and life, needing treatment

$\square$ IV - uncontrollable vomiting, daily > 5 times, needing hospitalisation

* To categorise into the following effectiveness categories:

$\square$ complete response (CR) - no nausea/vomiting (grade 0), good appetite

$\square$ partial response (PR) - daily vomiting 1-2 times (grade I ${ }^{\sim}$ II), slight reduced oral intake

$\square$ mild response (MR) - daily vomiting 2-5 times (II-III grade), obvious reduced oral intake

$\square$ no response (NC) - daily > 5 times vomiting, no appetite or thoughts of eating (grade III-IV)

- 2. Time of first vomiting episode (in days)

Secondary outcomes

Olanzapine for the prevention and treatment of cancer-related nausea and vomiting in adults (Review) 
Mao 2011 (Continued)

\section{- $\mathrm{AES}$}

Notes

Full paper translated and data extracted. However, this is a cross-over trial where participants sorted into the 2 groups $A$ and $B$, but regrouped into Intervention vs Control for analysis and outcome reporting. We were therefore unable to only extract first phase data. Awaiting further information from study authors

\begin{tabular}{ll}
\hline Methods & Trial method: RCT \\
& Randomised: yes \\
MEC or HEC: HEC or MEC \\
Blinding: Nil \\
Arms: 2 \\
Multicentre/single-centre: single \\
Dates: not stated
\end{tabular}

\section{Participants}

$$
\mathrm{N}=120(60 \text { per arm) }
$$

Age: 2 subgroups: $<60$ and $\geq 60$.

Number of people (Intervention group) vs (Control group) ( 27 vs 29 in < 60 group, 33 vs 31 in $\geq 60$ ). Paper does not state if all participants were over 18 years Gender: Male + Female

Inclusion criteria: $\mathrm{ECOG} \geq 2$; neutrophil count $\geq 1500 / \mu \mathrm{L}, \mathrm{Hb} \geq 10 \mathrm{~g} / \mathrm{L}$, platelet $\geq 80000 / \mu \mathrm{L}$, serum creatinine $\leq 1.5 \mathrm{mg} / \mathrm{dL}$, serum bilirubin was $<1.5 \mathrm{mg} / \mathrm{dL}$

Exclusion criteria: no brain metastases, no digestive tract obstruction and other primary disease that could cause nausea and vomiting. Participants without breast cancer. No history of mental illness. No abdominal radiation therapy at the same time. No known history of olanzapine allergy. No serious heart disease and diabetes. No other medical disease

Baseline characteristic: solid tumour

Other important effect modifiers, if applicable (e.g. radiotherapy): no abdominal radiotherapy Setting and location: hospital, China

Number of people screened: 120

Number of participants randomised - all: 120

Number randomised to each group: 60

Number receiving treatment as allocated: all

Number not receiving treatment as allocated: nil

Number dropped out: nil (no follow-up data for any outcome available)

Number excluded from analysis (for all outcomes): nil

Number completed: 120 the 1st day of chemotherapy until the end of chemotherapy. Olanzapine ( $2.5 \mathrm{mg}$, twice/day, oral) from day 1-5 of chemotherapy

Control group $\mathbf{B}(\mathrm{N}=60)$ : gamla SiQiong ( $3 \mathrm{mg}$ daily) + dexamethasone (5 mg daily IV) since the 1st day of chemotherapy until the end of chemotherapy

Use of additional treatments if any (co-interventions, additional analgesia): nil

\section{Outcomes}

\section{Primary outcomes}

- Complete response (CR) in the acute and delayed periods

- Effective response (ER) in the acute and delayed periods 
Meng 2016 (Continued)

Secondary outcomes

1. QOL during chemotherapy as measured by EORTC QLQ-C30

2. AEs

Notes

Awaiting information from authors regarding age of participants

Mukesh 2017

\begin{tabular}{|c|c|}
\hline Methods & $\begin{array}{l}\text { Trial method: RCT } \\
\text { MEC or HEC: HEC } \\
\text { Randomised: yes } \\
\text { Blinding: not stated } \\
\text { Arms: } 2 \\
\text { Multicentre/single-centre: not stated } \\
\text { Dates: not stated }\end{array}$ \\
\hline Participants & $\begin{array}{l}\mathrm{N}=84 \text { ( } 42 \text { in each arm) } \\
\text { Age: } 29-80 \\
\text { Gender: Female } \\
\text { Inclusion criteria: "breast cancer patients receiving doxorubicin } 60 \mathrm{mg} / \mathrm{m} 2 \text { and cyclophos- } \\
\text { phamide } 600 \mathrm{mg} / \mathrm{m} 2 \text { chemotherapy... chemotherapy naive; no nausea/ vomiting in the past } 24 \\
\text { hours were included" } \\
\text { Exclusion criteria: "patients with seizure disorder, brain metastasis, prior use of antipsychotic } \\
\text { agents and hypersensitivity to olanzapine were excluded." } \\
\text { Baseline characteristics: } \\
\text { Setting and location: not stated } \\
\text { Number of people screened: not stated } \\
\text { Number of participants randomised - all: not stated } \\
\text { Number randomised to each group: not stated } \\
\text { Number receiving treatment as allocated: not stated } \\
\text { Number not receiving treatment as allocated: not stated } \\
\text { Number dropped out: not stated } \\
\text { (no follow-up data for any outcome available) } \\
\text { Number excluded from analysis (for all outcomes): not stated } \\
\text { Number completed: not stated }\end{array}$ \\
\hline
\end{tabular}

Interventions

Intervention group $\mathbf{A}(\mathrm{N}=$ ?): olanzapine $10 \mathrm{mg}$ on day $1-3$, oral tablet

Intervention group $\mathbf{B}(\mathrm{N}=$ ?): aprepitant $125 \mathrm{mg}$ on day 1 and $80 \mathrm{mg}$ on days $2-3$, oral tablet

Use of additional treatments if any (co-interventions, additional analgesia): injection palnosteron $0.25 \mathrm{mg}$, injection dexamethasone $8 \mathrm{mg}$ on day 1 . Use of rescue therapy for nausea or vomiting was permitted

\section{Outcomes}

Primary outcomes

- Complete response (CR) for nausea that is no nausea in the acute (within 24 hours), delayed (day 2-5), and overall periods (0-120 hours)

Secondary outcomes

- CR for vomiting and no use of rescue drugs in all periods

Notes

Abstract only available. Awaiting further information from study authors

Funding: no information

Declaration of interest: no information 


\section{MEC or HEC: HEC}

Randomised: yes

Blinding: not stated

Arms: 2

Multicentre/single-centre: not stated

Dates: Jan 2013 - December 2015

\begin{tabular}{ll}
\hline Participants & N $=478$ \\
Age: (mean $=45.7$ years) \\
Gender: not stated \\
Inclusion criteria: not stated \\
Exclusion criteria: not stated \\
Baseline Characteristics: not stated \\
Setting and location: Vietnam, outpatients \\
Number of people screened: 358 \\
Number of participants randomised - all: 478 \\
Number randomised to each group: 239 \\
Number receiving treatment as allocated: not stated \\
Number not receiving treatment as allocated: not stated \\
Number dropped out: 0 \\
(no follow-up data for any outcome available) \\
Number excluded from analysis (for all outcomes): not stated \\
Number completed: not stated
\end{tabular}

Interventions

Intervention group $\mathbf{A}(\mathrm{N}=$ ?): olanzapine $10 \mathrm{mg}$ once/day + omeprazole $20 \mathrm{mg}$ once/day for 3 day Intervention group $\mathbf{B}(\mathrm{N}=$ ?): dexamethasone $4 \mathrm{mg}$ oral twice/day omeprazole $20 \mathrm{mg}$ oral twice/ day and metoclopramide $20 \mathrm{mg} 3$ times/day for 5 days

Use of additional treatments if any (co-interventions, additional analgesia):

\begin{tabular}{ll}
\hline Outcomes & Primary outcomes \\
& - Not stated \\
Secondary outcomes & Not stated \\
\hline Notes & Funding: no information \\
& Declaration of interest: no information \\
& Conference abstract only. Awaiting further information from study authors \\
\hline
\end{tabular}

Wang 2012

\begin{tabular}{ll}
\hline Methods & Not available \\
\hline Participants & Not available \\
\hline Interventions & Not available \\
\hline Outcomes & Not available \\
\hline \hline
\end{tabular}


Wang 2012 (Continued)

Notes

This article was unobtainable despite multiple attempts to source it

AEs: adverse events; ECG: electrocardiogram; ECOG: Eastern Cooperative Oncology Group; EORTC QLQ-C30: European Organisation for Research and Treatment of Cancer quality of life questionnaire; FLIE: Functional Living Index-Emesis; HEC: highly emetogenic chemotherapy; IV: intravenous; MEC: moderately emetogenic chemotherapy; NVAS: nausea visual analogue scale; QOL: quality of life

Characteristics of ongoing studies [ordered by study ID]

Abe 2017

Trial name or title

A randomised, double-blind, placebo-controlled phase iii study evaluating olanzapine $5 \mathrm{mg}$ combined with standard antiemetic therapy for the prevention of CINV in patients receiving cisplatin-based chemotherapy

\begin{tabular}{|c|c|}
\hline Methods & $\begin{array}{l}\text { Trial method: RCT } \\
\text { MEC or HEC: unclear } \\
\text { Randomised: yes } \\
\text { Blinding: not stated } \\
\text { Arms: } 2 \\
\text { Multicentre/single - centre: multi } \\
\text { Dates: Feb } 2017 \text { - not stated }\end{array}$ \\
\hline Participants & $\begin{array}{l}\mathrm{N}=\text { not stated } \\
\text { Age: not stated } \\
\text { Gender: not stated } \\
\text { Inclusion criteria: not stated } \\
\text { Exclusion criteria: not stated } \\
\text { Baseline Characteristics: not stated } \\
\text { Setting and location: Japan } \\
\text { Number of people screened: not stated } \\
\text { Number of participants randomised - all: not stated } \\
\text { Number randomised to each group: not stated } \\
\text { Number receiving treatment as allocated: not stated } \\
\text { Number not receiving treatment as allocated: not stated } \\
\text { Number dropped out: not stated } \\
\text { (no follow-up data for any outcome available) } \\
\text { Number excluded from analysis (for all outcomes): not stated } \\
\text { Number completed: not stated }\end{array}$ \\
\hline Interventions & $\begin{array}{l}\text { Intervention group } \mathbf{A}(\mathrm{N}=\text { ?): } 5 \mathrm{mg} \text { of olanzapine } \\
\text { Control group } \mathbf{B}(\mathrm{N}=\text { ?): placebo } \\
\text { Use of additional treatments if any (co-interventions, additional analgesia): palonosetron (PALO), } \\
\text { aprepitant (APR), and dexamethasone (DEX) }\end{array}$ \\
\hline Outcomes & $\begin{array}{l}\text { Primary outcomes } \\
\text { - Complete response rate in the delayed phase } \\
\text { Secondary outcomes } \\
\text { - Complete response during acute ( } 0-24 \text { hours) and overall phases ( } 0-120 \text { hours) are complete and } \\
\text { total control rates during each phase } \\
\text { - Complete response during overall phases ( } 0 \text {-120 hours) } \\
\text { - Total control during acute ( } 0-24 \text { hours) }\end{array}$ \\
\hline
\end{tabular}


Abe 2017 (Continued)

Starting date

Feb 2017

Contact information ma.abe@scchr.jp

Notes

\section{ChicTR-TTRCC-14004093}

Trial name or title

Olanzapine versus aprepitant for the prevention of high-dose cisplatin-induced nausea and vomiting: a randomised phase III trial

\begin{tabular}{ll}
\hline Methods & Not stated \\
\hline Participants & Not stated \\
\hline Interventions & Not stated \\
\hline Outcomes & Not stated \\
\hline Starting date & Not stated \\
\hline Contact information & Not stated \\
\hline Notes & \\
\hline
\end{tabular}

Hashimoto 2017

Trial name or title

1607TiP - J-FORCE study: a randomized, double-blind, placebo-controlled phase III study evaluating olanzapine ( $5 \mathrm{mg}$ ) combined with standard antiemetic therapy

\begin{tabular}{ll}
\hline Methods & Trial method: RCT \\
MEC or HEC: HEC \\
Randomised: yes \\
Blinding: not stated \\
Arms: 2 \\
Multicentre/single-centre: not stated \\
Dates: not stated \\
N $=690$ \\
Age: 20 -75 years \\
Gender: not stated \\
Inclusion criteria: ECOG performance status between $0-2$, and have malignant disease, sched- \\
uled to receive HEC with cisplatin at a dose $\geq 50 \mathrm{mg} / \mathrm{m} 2$ \\
Exclusion criteria: having diabetes mellitus or being treated with antipsychotic agents within 48 \\
hours before enrolment \\
Baseline Characteristics: not stated \\
Setting and location: not stated \\
Number of people screened: not stated \\
Number of participants randomised - all: not stated \\
Number randomised to each group: not stated \\
Number receiving treatment as allocated: not stated \\
Number not receiving treatment as allocated: not stated
\end{tabular}


Hashimoto 2017 (Continued)

Number dropped out: not stated

(no follow-up data for any outcome available)

Number excluded from analysis (for all outcomes): not stated

Number completed: not stated

\begin{tabular}{|c|c|}
\hline Interventions & $\begin{array}{l}\text { Intervention group } \mathbf{A}(\mathrm{N}=\text { ?): } 5 \mathrm{mg} \text { olanzapine } \\
\text { Control group } \mathbf{B}(\mathrm{N}=\text { ?): placebo olanzapine } \\
\text { Use of additional treatments if any (co-interventions, additional analgesia): Aprepitant (125 mg } \\
\text { oral on day } 1,80 \mathrm{mg} \text { oral on day } 2-3) \text {, palonosetron ( } 0.75 \mathrm{mg} \text { IV on day } 1 \text { ) and dexamethasone (9.9 } \\
\mathrm{mg} \text { IV on day } 1 \text { and } 6.6 \mathrm{mg} \text { IV on day } 2-4)\end{array}$ \\
\hline Outcomes & $\begin{array}{l}\text { Primary outcomes } \\
\text { - Complete response, defined as no emetic episodes and without the use of rescue medications in } \\
\text { the delayed phase (24-120 hours) } \\
\text { Secondary outcomes } \\
\text { - Complete response during acute (0-24 hours) } \\
\text { - Complete response overall phases (0-120 hours) } \\
\text { - Complete and total control rates } \\
\text { - Level of nausea, appetite and somnolence }\end{array}$ \\
\hline
\end{tabular}

\begin{tabular}{ll}
\hline Starting date & Not stated \\
\hline Contact information & winnie@clo.cuhk.edu.hk
\end{tabular}

Notes

\section{JPRN-UMIN000010317}

\section{Trial name or title}

Efficacy of olanzapine for relief of nausea with incomplete bowel obstruction in advanced cancer patient: pragmatic randomized controlled trial, JPRN-UMIN000010317

$\begin{array}{ll}\text { Methods } & \text { Trial method: RCT } \\ \text { MEC or HEC: not applicable } \\ \text { Randomised: yes } \\ \text { Blinding: not stated } \\ \text { Arms: } 2 \\ \text { Multicentre/single-centre: not stated } \\ \text { Dates: not stated }\end{array}$

Participants

$\mathrm{N}=$ not stated

Age: 20 and older

Gender: Male + Female

Inclusion criteria: metastatic or locally advanced cancer; incomplete bowel obstruction due to malignant tumour; NRS of nausea $\geq 4$; nausea not due to hypercalcaemia, brain hypertension or drugs; able to take oral medication; aged $\geq 20$ years at the time of obtaining informed consent; agree to participate with written consent under a sufficient explanation and understanding. Hospitalised patients

Exclusion criteria: received chemotherapy or radiation therapy within 28 day; diagnosed diabetes mellitus or having history of diabetes mellitus (possibility of worsening diabetes mellitus). Diagnosis of diabetes mellitus is on the basis of 1 . fasting blood sugar $\geq 126 \mathrm{mg} / \mathrm{dL}, 2$. 2-hour blood sugar level after 75 g glucose tolerance test $\geq 200 \mathrm{mg} / \mathrm{dL}$ or 3 . random blood sugar $\geq 200 \mathrm{mg} / \mathrm{dL}$ and $\mathrm{HbAlC} \geq 6.5 \%$. Patients administrated with dopaminergic drugs such as phenothiazine, buty- 
rophenone and atypical antipsychotic within 3 days; with nasogastric tube, percutaneous endoscopic gastrostomy and percutaneous trans-oesophageal gastro-tubing; with indication of surgery or chemotherapy; with bowel obstruction due to non-malignant disease; with complete bowel obstruction; with signs of gastrointestinal tract perforation or sepsis; with incidence of colic pain (paroxysmal and repetitive abdominal pain) within 48 hours; with high risk of colic pain according to metoclopramide; judged to be inappropriate for the study by the responsible researcher; lacking in ability to make suitable decisions; judged to be inappropriate for the study by the physician Baseline characteristics: not stated Setting and location: not stated Number of people screened: not stated Number of participants randomised - all: not stated Number randomised to each group: not stated Number receiving treatment as allocated: not stated Number not receiving treatment as allocated: not stated Number dropped out: not stated (no follow-up data for any outcome available) Number excluded from analysis (for all outcomes): not stated Number completed: not stated

Intervention group $\mathbf{A}(\mathrm{N}=$ ?): olanzapine $(5 \mathrm{mg})$ or tablet $5 \mathrm{mg} /$ day (evening) orally Intervention group $\mathbf{B}(\mathrm{N}=$ ?): 1) metoclopramide $(5 \mathrm{mg})$ tablet $30 \mathrm{mg} /$ day (morning/noon/ evening) orally or 2) metoclopramide $(10 \mathrm{mg}$ ) injectable solution $20 \mathrm{mg} /$ day (intermittent or continuous) subcutaneous or drip infusion

Use of additional treatments if any (co-interventions, additional analgesia): not stated

\begin{tabular}{ll}
\hline Outcomes & Primary outcomes \\
& C Changes in the average value of nausea for 24 hours with NRS \\
Secondary outcomes & $\begin{array}{l}\text { Proportion of participants with average value of nausea reduced }>30 \% \text { in NRS, number of vomit, } \\
\text { satisfaction rating and intention to continue treatment }\end{array}$ \\
\hline Starting date & Not stated \\
\hline Contact information & kaneishi@tkn-hosp.gr.jp \\
\hline Notes & \\
\hline
\end{tabular}

\section{Mukhopadhyay 2017a}

\begin{tabular}{ll}
\hline Trial name or title & $\begin{array}{l}\text { Low dose vs. standard dose adjuvant olanzapine in chemotherapy induced nausea and vomiting: a } \\
\text { prospective, randomized, double blinded, controlled study }\end{array}$ \\
\hline Methods & Trial method: RCT, parallel-arm \\
& MEC or HEC: MEC and HEC \\
& Randomised: yes \\
& Blinding: double \\
& Arms: 2 \\
& Multicentre/single-centre: not stated \\
& Dates: not stated \\
\hline Participants & $\mathrm{N}=100$ \\
& Age: not stated
\end{tabular}


Mukhopadhyay 2017a (Continued)

Gender: not stated

Inclusion criteria: not stated

Exclusion criteria: not stated

Baseline characteristics: not stated

Setting and location: not stated

Number of people screened: not stated

Number of participants randomised - all: not stated

Number randomised to each group: not stated

Number receiving treatment as allocated: not stated

Number not receiving treatment as allocated: not stated

Number dropped out: not stated

(no follow-up data for any outcome available)

Number excluded from analysis (for all outcomes): not stated

Number completed: not stated

Interventions

Intervention group A ( $\mathrm{N}=$ ?): olanzapine $5 \mathrm{mg}$ ondansetron $16 \mathrm{mg}$ on day 1 and dexamethasone 8 $\mathrm{mg}$ from day $1-3$

Intervention group $\mathbf{B}(\mathrm{N}=$ ?): olanzapine $10 \mathrm{mg}$ ondansetron $16 \mathrm{mg}$ on day 1 and dexamethasone 8 $\mathrm{mg}$ from day $1-3$

Use of additional treatments if any (co-interventions, additional analgesia):

\section{Outcomes}

Primary outcomes

- CINV in acute, delayed and overall 1 - 5 day was measured along with day-time sedation

- QOL before and after chemotherapy was measured by FACT questionnaire

Secondary outcomes

- Not stated

\begin{tabular}{ll}
\hline Starting date & Not stated \\
\hline Contact information & sandipcmcl@gmail.com \\
\hline Notes & Funding: no information \\
& Declaration of interest: no information \\
\hline
\end{tabular}

Nagashima et al 2015

\begin{tabular}{ll}
\hline Trial name or title & $\begin{array}{l}\text { A double-blind randomized Phase II study of olanzapine } 10 \mathrm{mg} \text { versus } 5 \text { mg for emesis induced by } \\
\text { highly emetogenic chemotherapy }\end{array}$ \\
\hline Methods & Trial method: RCT \\
& MEC or HEC: HEC \\
& Randomised: yes \\
& Blinding: double blind \\
& Arms: 2 \\
& Multicentre/single-centre: 9 centre \\
& Dates: ongoing \\
\hline Participants & Age: 150 \\
Gender: 20 Male + Female \\
Inclusion criteria: solid malignant tumour
\end{tabular}


Exclusion criteria: no previous HEC, ECOG performance status 0-2, brain metastases excluded Baseline characteristics: not stated

Setting and location: hospitals in Japan

Number of people screened: not stated

Number of participants randomised - all: not stated

Number randomised to each group: not stated

Number receiving treatment as allocated: not stated

Number not receiving treatment as allocated: not stated

Number dropped out: not stated

(no follow-up data for any outcome available)

Number excluded from analysis (for all outcomes): not stated

Number completed: not stated

\begin{tabular}{|c|c|}
\hline Interventions & $\begin{array}{l}\text { Intervention group } \mathbf{A}(\mathrm{N}=\text { ?): olanzapine } 5 \mathrm{mg} \text { oral daily crushed in a powder with lactose on day } \\
1-4 \\
\text { Intervention group } \mathbf{B}(\mathrm{N}=\text { ?): olanzapine } 10 \mathrm{mg} \text { oral daily crushed in a powder with lactose on day } \\
1-4 \\
\text { Use of additional treatments if any (co-interventions, additional analgesia): Not stated }\end{array}$ \\
\hline Outcomes & $\begin{array}{l}\text { Primary outcomes } \\
\text { - Complete Response (no vomiting and no rescue medications in delayed phase (day 2-5)) } \\
\text { Secondary outcomes } \\
\text { - Complete Response (no vomiting and no rescue medications in acute phase (day } 1 \text { )) } \\
\text { - Complete Response (no vomiting and no rescue medications in overall phase (day 1-5)) } \\
\text { - Complete control (no vomiting, no rescue medication, no nausea or mild nausea) in acute, de- } \\
\text { - layed and overall phases }\end{array}$ \\
\hline Starting date & Not stated \\
\hline Contact information & nbryamam@ncc.go.jp \\
\hline
\end{tabular}

Notes

\section{NCT02290470}

Trial name or title

Olanzapine against delayed nausea and vomiting in women receiving carboplatin plus paclitaxel NCT02290470

Methods

Trial method: RCT

MEC or HEC: HEC

Randomised: yes

Blinding: not stated

Arms: not stated

Multicentre/single-centre: not stated

Dates: not stated
$\mathrm{N}=$ not stated

Age: $\geq 18$ years

Gender: Female

Inclusion criteria: histologically or cytologically documented gynaecologic cancer 
patients who are chemotherapy naive and scheduled to receive 1-day MEC (carboplatin Area under Curve (AUC) 5 plus paclitaxel). ECOG Performance Status of 0-2. Adequate organ system function, defined as follows: bone marrow: absolute neutrophil count $\geq 1,500 / \mathrm{L}$, platelets $\geq 100,000 / \mathrm{L}$ liver: bilirubin 1.5 x upper limit of normal (ULN); transaminases $\leq 2.5 \times$ ULN kidney: creatinine $<=1.5$ $x$ ULN. Able to take oral medications

Exclusion criteria: psychiatric illness or social situation that would preclude study compliance. History of CNS (e.g. brain metastases, seizure disorder) Positive pregnancy test just before registration.

Treatment with any antiemetic medication from 24 hours -5 days after treatment. Treatment with another antipsychotic agent such as risperidone, quetiapine, clozapine, phenothiazine, or butyrophenone for 30 days before or during protocol therapy. Concurrent abdominal radiation therapy. Concurrent quinolone antibiotic therapy. Known hypersensitivity to olanzapine. Vomiting and/ or significant nausea ( $\geq$ Common Toxicity Criteria for Adverse Events (CTCAE) grade 2 ) within the 24 hours before beginning chemotherapy. Another organic cause for nausea or vomiting unrelated to chemotherapy administration. Chronic alcoholism (as determined by the investigator). Known cardiac arrhythmia, uncontrolled congestive heart failure or acute myocardial infarction within the previous 6 months. History of uncontrolled diabetes mellitus

Baseline characteristics: not stated

Setting and location: not stated

Number of people screened: not stated

Number of participants randomised - all: not stated

Number randomised to each group: not stated

Number receiving treatment as allocated: not stated

Number not receiving treatment as allocated: not stated

Number dropped out: not stated

(no follow-up data for any outcome available)

Number excluded from analysis (for all outcomes): not stated

Number completed: not stated
Intervention group A ( $\mathrm{N}=$ ?): dexamethasone (16 mg IV on the day of chemotherapy), plus olanzapine (10 mg orally on the day of chemotherapy and $10 \mathrm{mg}$ orally on day 2, 3 post chemotherapy) Intervention group $\mathbf{B}(\mathrm{N}=$ ?): dexamethasone (16 mg IV on the day of chemotherapy and $4 \mathrm{mg}$ orally day 2,3 post chemotherapy), plus olanzapine (10 $\mathrm{mg}$ orally on the day of chemotherapy and $10 \mathrm{mg}$ orally on day 2,3 post chemotherapy)

Intervention Group $\mathbf{C}(\mathrm{N}=$ ?): dexamethasone $16 \mathrm{mg}$ IV on the day of chemotherapy (day 1), plus dexamethasone $8 \mathrm{mg}$ orally on day 2 and 3 post chemotherapy

Use of additional treatments if any (co-interventions, additional analgesia): palonosetron ( 0.25 $\mathrm{mg}$ IV) on the day of chemotherapy plus dexamethasone (16 mg IV on the day of chemotherapy and 8 or $4 \mathrm{mg}$ (depending on the experimental arm) oral on day 2 and 3 post-chemotherapy)

Outcomes

Primary outcomes

- Complete protection (time frame: day 2-5 post-chemotherapy). Proportion of patients achieving delayed Complete Protection, defined as no vomiting, no rescue antiemetics, and no more than mild nausea measured by the Nausea and Vomiting Daily Diary/Questionnaire.

Secondary outcomes

- Nausea scores (time frame: up to 5 days) measured by the Nausea and Vomiting Daily Diary/Questionnaire

Others:

- proportion of participants achieving Complete Response (time frame: up to 5 day) defined as no emetic episodes and no use of rescue antiemetics measured by the Nausea and Vomiting Daily Diary/Questionnaire

- Impact of nausea and vomiting on daily life activities (time frame: day 1 (pre-chemotherapy) and day 6 (post-chemotherapy)) as measured by the Functional Living Index-Emesis Questionnaire

- Incidence of potential toxicities related to olanzapine (time frame: up to 5 days) as measured by the Nausea and Vomiting Daily Diary/Questionnaire. 
NCT02290470 (Continued)

- Frequency of rescue antiemetics (time frame: up to 5 days) measured by the Nausea and Vomiting Daily Diary/Questionnaire

\begin{tabular}{ll}
\hline Starting date & Not stated \\
\hline Contact information & luigi.celio@istitutotumori.mi.it \\
\hline Notes &
\end{tabular}

Trial name or title

Methods
A randomized study of olanzapine for the prevention of CINV in patients receiving moderately emetogenic chemotherapy NCT02400866

Trial method: RCT

MEC or HEC: MEC

Randomised: yes

Blinding: double - blind

Arms: 2

Multicentre/single - centre: not stated

Dates: not stated

\section{Participants}

\author{
$\mathrm{N}=$ not stated \\ Age: $\geq 19$ years \\ Gender: Male + Female
}

Inclusion criteria: no history of receiving MEC/HEC during last 6 months, and is to receive a first course of MEC including $\geq 1$ of following agents: carboplatin, cyclophosphamide $\leq 1,500 \mathrm{mg} / \mathrm{m} 2$, daunorubicin, doxorubicin $<60 \mathrm{mg} / \mathrm{m} 2$, epirubicin $\leq 90 \mathrm{mg} / \mathrm{m2}$, irinotecan, oxaliplatin, melphalan, methotrexate $\geq 250 \mathrm{mg} / \mathrm{m} 2$. ECOG performance status 0 -2. Predicted life expectancy $\geq 3$ months. Adequate bone marrow, kidney, and liver function as evidenced by: neutrophils $\geq 1,500 / \mathrm{mm} 3$, platelet count $\geq 100,000 / \mathrm{mm} 3$, total bilirubin $\leq 2 \times U L N$, aspartate aminotransferase $\leq 3 \times U L N$, ALT $\leq 3 \times$ ULN (for subjects with known liver metastases, total bilirubin $\leq 3 \times U L N, A S T \leq 5 \times$ ULN, ALT $\leq$ $5 \times$ ULN), creatinine $\leq 1.5 \times$ ULN or Creatinine Clearance $\geq 50 \mathrm{ml} / \mathrm{min}$. No episodes of nausea and vomiting during last 24 hours before enrolment. Participant provides written informed consent. Exclusion criteria: people with uncontrolled neuro-psychiatric disease (alcohol abuse, seizure, psychosis etc) except malignant tumour. Scheduled to receive HEC agents. Contraindication to the administration of palonosetron, dexamethasone, and olanzapine due to hypersensitivity or any other reasons. Severe cognitive impairment. Symptomatic or uncontrolled brain metastasis or brain tumour. Female participants of childbearing potential who do not agree to use a proper contraceptive method or to limit breast feeding. Taken the following agents: risperidone, quetiapine, clozapine, phenothiazine, butyrophenone, 5-HT3 antagonist, benzamide, domperidone, cannabinoids, NK1 antagonist, benzodiazepines. Plans to receive other chemotherapy, abdominal radiation, surgery, or immunotherapy. Any history of arrhythmia, uncontrolled congestive heart failure, acute myocardial infarction during last 6 months. History of uncontrolled diabetes. Used any investigational drugs within 30 days of randomisation.

\section{Baseline characteristics: not stated}

Setting and location: not stated

Number of people screened: not stated

Number of participants randomised - all: not stated

Number randomised to each group: not stated

Number receiving treatment as allocated: not stated

Number not receiving treatment as allocated: not stated

Number dropped out: not stated

(no follow-up data for any outcome available)

Number excluded from analysis (for all outcomes): not stated 
NCT02400866 (Continued)

Number completed: not stated
Interventions Intervention group $\mathbf{A}(\mathrm{N}=$ ?): olanzapine

Control group $\mathbf{B}(\mathrm{N}=$ ?): placebo

Use of additional treatments if any (co-interventions, additional analgesia): palonosetron + dexamethasone

\section{Primary outcomes}

- Complete response rate for the acute phase (0-24 hours) after chemotherapy (time frame: during 24 hours after first cycle of MEC)

\section{Secondary outcomes}

- Complete response rate for the delayed phase (24-120 hours) and overall phase (0-120 hours) after chemotherapy (time frame: during 0-120 hours after first cycle of MEC)

- No vomiting for the overall phase (time frame: during 0-120 hours after first cycle of MEC)

- Significant emesis for the overall phase (time frame: during 0-120 hours after first cycle of MEC)

- Numbers and time for rescue medications (time frame: during 0-120 hours after first cycle of MEC)

Others

- Effects on quality of life by FLIE questionnaire (time frame: during 0-120 hours after first cycle of MEC)

\begin{tabular}{ll}
\hline Starting date & Not stated \\
\hline Contact information & poppoya99@naver.com \\
\hline
\end{tabular}

\section{Notes}

\section{NCT02635984}

Trial name or title

Study of FOND Versus FOND+O for the Prevention of CINV in hematology patients receiving highly emetogenic chemotherapy regimens (FOND-O), NCT02635984

\begin{tabular}{ll}
\hline Methods & Trial method: RCT \\
MEC or HEC: HEC +/- total body irradiation \\
Randomised: yes \\
Blinding: yes \\
Arms: 2 \\
Multicentre/single-centre: not stated \\
Dates: not stated \\
N $=$ not stated \\
Age: $\geq 18$ \\
Gender: Male + Female \\
Inclusion criteria: \\
Inpatient or outpatient haematology patient \\
Chemotherapy for hematologic malignancy \\
Conditioning therapy for stem cell transplantation: chemotherapy or Total Body Irradiation (TBI) \\
Exclusion criteria: allergy to olanzapine. Documented nausea or vomiting $\leq 24$ hours prior to en- \\
rolment. Treatment with other antipsychotic agents such as risperidone, quetiapine, clozapine, \\
phenothiazine or butyrophenone $\leq 30$ days prior to enrolment or planned during protocol therapy. \\
Chronic alcoholism. Pregnant. Declined or unable to provide an informed consent
\end{tabular}

Olanzapine for the prevention and treatment of cancer-related nausea and vomiting in adults (Review) 
NCT02635984 (Continued)

Baseline characteristics: not stated
Setting and location: not stated
Number of people screened: not stated
Number of participants randomised - all: not stated
Number randomised to each group: not stated
Number receiving treatment as allocated: not stated
Number not receiving treatment as allocated: not stated
Number dropped out: not stated
(no follow-up data for any outcome available)
Number excluded from analysis (for all outcomes): not stated
Number completed: not stated

Interventions

Intervention group $\mathbf{A}(\mathrm{N}=$ ?): olanzapine $10 \mathrm{mg}$ once/day for 3 days

Control group $\mathbf{B}(\mathrm{N}=$ ?): placebo once/day for 3 days

Use of additional treatments if any (co-interventions, additional analgesia): ondansetron and dexamethasone on each day of chemotherapy plus fosaprepitant $150 \mathrm{mg}$ IV once

\section{Primary outcomes}

- Overall percentage of participants who had a complete response defined as no emesis and minimal nausea ( $<25 \mathrm{~mm}$ on a $100 \mathrm{~mm}$ VAS) during the overall assessment period (starting day 1 of chemotherapy and continuing for 5 days after discontinuation of chemotherapy) for the first cycle of chemotherapy.

Secondary outcomes

- Number of emetic episodes per participant. To be reported as acute (chemotherapy days), delayed ( 5 days after chemotherapy administration), and overall phases (chemotherapy days plus 5 days after)

- Number of rescue medications doses administered per participant. To be reported as acute (chemotherapy days), delayed (5 days after chemotherapy administration), and overall phases (chemotherapy days plus 5 days after)

- Percent of participants with no significant nausea. To be reported as acute (chemotherapy days), delayed ( 5 days after chemotherapy administration), and overall phases (chemotherapy days plus 5 days after)

- Percent of participants achieving complete protection (CP = no emesis, no breakthrough antiemetic use, no significant nausea). To be reported as acute (chemotherapy days), delayed (5 days after chemotherapy administration), and overall phases (chemotherapy days plus 5 days after)

Others:

- Rate of discontinuation of study drug. To be reported as acute (chemotherapy days), delayed (5 days after chemotherapy administration), and overall phases (chemotherapy days plus 5 days after)

\section{Not stated}


NCT02939287 (Continued)

\section{MEC or HEC: HEC}

Randomised: yes

Blinding: nil

Arms: 2

Multicentre/single-centre: not stated

Dates: not stated

Narticipants $\quad$ Age: $18-80$ years
Gender: not stated
Inclusion criteria: autologous transplant containing high-dose melphalan as part of the condi-
tioning chemotherapy regimen. Able to tolerate oral medications.
Exclusion criteria: nausea/vomiting within 12 hours before planned high-dose conditioning
chemotherapy. Any antiemetic treatment within 24 hours before planned high dose conditioning
chemotherapy. Pregnancy. Baseline corrected QT interval (QTC) $>500$ ms. History of seizures. Histo-
ry of CNS disease. HIV
Baseline characteristics: not stated
Setting and location: not stated
Number of people screened: not stated
Number of participants randomised - all: not stated
Number randomised to each group: not stated
Number receiving treatment as allocated: not stated
Number not receiving treatment as allocated: not stated
Number dropped out: not stated
(no follow-up data for any outcome available)
Number excluded from analysis (for all outcomes): not stated
Number completed: not stated

$\begin{array}{ll}\text { Interventions } & \text { Intervention group } \mathbf{A}(\mathrm{N}=\text { ?): aprepitant } \\ \text { Intervention group } \mathbf{B}(\mathrm{N}=\text { ?): olanzapine } \\ \text { Use of additional treatments if any (co-interventions, additional analgesia): standard antiemetic } \\ \text { regime }\end{array}$

\section{Outcomes}

Primary outcomes

- CR (time frame: within 120 hours following melphalan administration) no emesis and no rescue antiemetic therapy

Secondary outcomes

- Acute CR (time frame: 0-24 hours) no emesis or rescue therapy

- Delayed CR (time frame: 25-120 hours) no emesis or rescue therapy

- Very delayed CR (time frame: 121-168 hours) no emesis or rescue therapy

- Mucositis/significant mucositis (time frame: up to 14 days)

Others:

- Time to neutrophil engraftment (time frame: up to 14 days)

- Time to platelet engraftment (time frame: up to 30 days)

\begin{tabular}{ll}
\hline Starting date & Not stated \\
\hline Contact information & Not stated \\
\hline Notes & \\
\hline
\end{tabular}



of CIN NCT02970643

\begin{tabular}{|c|c|}
\hline Methods & $\begin{array}{l}\text { Trial method: single-group assignment } \\
\text { MEC or HEC: MEC } \\
\text { Randomised: not stated } \\
\text { Blinding: no } \\
\text { Arms: not stated } \\
\text { Multicentre/single-centre: not stated } \\
\text { Dates: July } 2016 \text { - December } 2017\end{array}$ \\
\hline Participants & $\begin{array}{l}\mathrm{N}=\text { not stated } \\
\text { Age: } \geq 18 \text { years } \\
\text { Gender: Male + Female } \\
\text { Inclusion criteria: ECOG performance status of } 0,1 \text {, or } 2 \text {, no severe cognitive compromise. Mod- } \\
\text { erate risk chemotherapy induced chemotherapy induced nausea and vomiting in 1st cycle. Con- } \\
\text { firmed histology } \\
\text { Exclusion criteria: ECOG performance status of } 3 \text { and } 4 \text {. Nausea or vomiting in the } 24 \text { hours before } \\
\text { enrolment. History of Nausea or vomiting Grade } 3 \text { before previous chemotherapy. Known history of } \\
\text { CNS disease (e.g. brain metastases or a seizure disorder). Bowel obstruction. Serum creatinine lev- } \\
\text { el of } 2.0 \text { mg per dL (177 } \mu \text { mol per L) or more. Aspartate or alanine aminotransferase level that was } \\
\text { > } 3 \text { times the ULN range. Treatment with another antipsychotic agent such as risperidone, quetiap- } \\
\text { ine, clozapine, a phenothiazine, or a butyrophenone within } 30 \text { days before enrolment. Treatment } \\
\text { with another antiemetic agent before } 48 \text { hours before enrolment. Uncontrolled severe infection } \\
\text { or uncontrolled severe comorbidity. Concurrent abdominal radiotherapy. Known hypersensitivity } \\
\text { to olanzapine, palonosetron. Known cardiac arrhythmia, uncontrolled congestive heart failure, or } \\
\text { acute myocardial infarction within the previous } 6 \text { months. } \\
\text { Baseline characteristics: not stated } \\
\text { Setting and location: not stated } \\
\text { Number of people screened: not stated } \\
\text { Number of participants randomised - all: not stated } \\
\text { Number randomised to each group: not stated } \\
\text { Number receiving treatment as allocated: not stated } \\
\text { Number not receiving treatment as allocated: not stated } \\
\text { Number dropped out: not stated } \\
\text { (no follow-up data for any outcome available) } \\
\text { Number excluded from analysis (for all outcomes): not stated } \\
\text { Number completed: not stated }\end{array}$ \\
\hline
\end{tabular}

Use of additional treatments if any (co-interventions, additional analgesia): not stated

\begin{tabular}{ll}
\hline Outcomes & Primary outcomes \\
& Not stated \\
& Secondary outcomes \\
& Not stated \\
\hline Starting date & Not stated \\
\hline Contact information & Not stated \\
\hline
\end{tabular}

Notes 
MEC or HEC: randomised: yes

Blinding: no

Arms: not stated

Multicentre/single - centre: not stated

Dates: not stated

Inclusion criteria: Chinese patients, female $\geq 18$ and $<75$ years of age, diagnosed with early breast cancer; naive to MEC/HEC; scheduled to receive 1st course of adjuvant chemotherapy for breast cancer follows: IV adriamycin $60 \mathrm{mg} / \mathrm{m} 2$ + cyclophosphamide $600 \mathrm{mg} / \mathrm{m} 2$. Predicted life expectancy of 4 months. ECOG Performance Status of 0-1. Premenopausal female participants must not be pregnant (documented negative urine pregnancy test). Must be able to read, understand and complete study questionnaires and diary, including questions requiring a VAS response and understand the procedures and agree to participate in the study by giving written informed consent Exclusion criteria: patient with advanced breast cancer; receiving cisplatin or any other chemotherapy of higher emetogenic potential, except for cyclophosphamide and doxorubicin in the regimens described above; scheduled to receive concurrent radiation as part of their chemotherapy regimen for their malignancy; experience any vomiting or grade 2-3 nausea per Common Terminology Criteria for Adverse Events version 4.0 (CTCAE v 4.03) in the 24 hours before day 1 of chemotherapy. History of treatment with MEC/HEC; active infection (e.g. pneumonia, systemic fungal infection) or any uncontrolled disease (e.g. diabetes mellitus, hypertension) which, in the opinion of the investigator, might confound the results of the study or pose unwarranted risk; history of glaucoma, dementia, seizures, Parkinson's disease, Neuroleptic Malignant Syndrome (NMS), thromboembolic events; currently uses any illicit drugs, including marijuana, or has current evidence of alcohol abuse as determined by the investigator; mentally incapacitated or has a significant emotional or psychiatric disorder that, in the opinion of the investigator, precludes study entry; regular alcohol drinker or smoker; history of any illness that, in the opinion of the investigator, might confound the results of the study or pose unwarranted risk; history of hypersensitivity to aprepitant, ondansetron or dexamethasone; phenylketonuria and abnormal uric acid. Any investigational drugs taken within 4 weeks prior to day 1 of cycle 1 , and/or is scheduled to receive any investigational drug during the study; taking systemic corticosteroid therapy at any dose; however, topical and inhaled corticosteroids are permitted; has taken a non-registered investigational drug within the 28 days of the Prestudy Visit. Use, in the 28 days prior to treatment day 1 , of barbiturates, rifampicin or rifabutin, phenytoin or carbamazepine. Use, in the 7 days prior to treatment day 1 , of terfenadine, cisapride, astemizole, clarithromycin (azithromycin, erythromycin and roxithromycin are permitted), ketoconazole or itraconazole (fluconazole is permitted), amifostine pimozide 5-HT3 antagonists (ondansetron, granisetron, dolasetron, or tropisetron) phenothiazines (e.g. prochlorperazine, fluphenazine, perphenazine, thiethylperazine, or chlorpromazine) butyrophenones (e.g. haloperidol or droperidol) benzamides (e.g. metoclopramide or alizapride) domperidone cannabinoids NK1 receptor antagonists. Use, in the 48 hours prior to treatment day 1 , of benzodiazepines or opiates, except for single daily doses of lorazepam. Use of the following drugs: carbamazepine fluvoxamine ciprofloxacin dopamine agonists. Antiparkinsonian medicinal products medicinal products known to increase QTc interval. Abnormal laboratory values.

\section{Baseline characteristics: not stated}

Setting and location: not stated

Number of people screened: not stated

Number of participants randomised - all: not stated

Number randomised to each group: not stated

Number receiving treatment as allocated: not stated

Number not receiving treatment as allocated: not stated

Number dropped out: not stated 
NCT03079219 (Continued)

(no follow-up data for any outcome available)

Number excluded from analysis (for all outcomes): not stated

Number completed: not stated

Interventions

Intervention group $\mathbf{A}(\mathrm{N}=$ ?):

Aprepitant day 1: $125 \mathrm{mg}$ daily; day 2-3: $80 \mathrm{mg}$ daily

Ondansetron day 1: $8 \mathrm{mg}$ twice/day

Dexamethasone day 1: $12 \mathrm{mg}$ daily

Olanzapine day 1-5: $10 \mathrm{mg}$ daily

Intervention group $\mathrm{B}(\mathrm{N}=$ ?):

Aprepitant day 1: $125 \mathrm{mg}$ daily, day 2-3: $80 \mathrm{mg}$ daily

Ondansetron day 1: $8 \mathrm{mg}$ twice/day

Dexamethasone day 1: $12 \mathrm{mg}$ daily; day 2-3: $4 \mathrm{mg}$ twice/day

Use of additional treatments if any (co-interventions, additional analgesia): not stated

Primary outcomes
- Number of episodes of nausea in the first 120 hours after first cycle of chemotherapy. (Time frame:
120 hours)
- Number of episodes of vomiting in the first 120 hours after first cycle of chemotherapy. (Time
frame: 120 hours)
Secondary outcomes
- Number of episodes of nausea in the first 120 hours during 4 cycles of chemotherapy. (Time frame:
120 hours)
- Number of episodes of vomiting in the first 120 hours during 4 cycles of chemotherapy. (Time
frame: 120 hours)

\begin{tabular}{ll}
\hline Starting date & Not stated \\
\hline Contact information & winnie@clo.cuhk.edu.hk \\
\hline
\end{tabular}

Notes

NCT03137121

$\begin{array}{ll}\text { Trial name or title } & \text { Olanzapine for the treatment of chronic nausea and/or vomiting in advanced cancer patients } \\ \text { NCT03137121 }\end{array}$

Methods Trial method: parallel-group

MEC or HEC: not stated

Randomised: yes

Blinding: double

Arms: 2

Multicentre/single-centre: not stated

Dates: April 2017- not stated

\begin{tabular}{ll}
\hline Participants & Age: $\geq 18$ years \\
Gender: Male + Female \\
Inclusion criteria: have histologically or cytologically-confirmed malignant disease in an ad- \\
vanced incurable stage. Have not received chemotherapy or radiation for $>14$ days (advanced can-
\end{tabular}


cer patients receiving hormonal therapy or targeted therapy that does not come with a recommendation for prophylactic antiemetic therapy are eligible). Have chronic nausea that has been present for $\geq 1$ week (worst daily score $>3,0-10$ VAS) or vomiting $\geq 5$ times over past week. Have serum creatinine $<2.0 \mathrm{mg} / \mathrm{dL}$ and SGOT or SGPT $<3$ times ULN $\leq 120 \mathrm{~d}$ prior to registration. ANC $>1500 \mathrm{~mm} 3$ $<120$ days prior to registration. Women of childbearing potential must consent to use adequate contraception throughout protocol therapy; women of childbearing potential must have a negative urine pregnancy test $<7$ days prior to registration.

Exclusion criteria: not be receiving treatment with another antipsychotic agent such as risperidone, quetiapine, clozapine, phenothiazine or butyrophenone for $\leq 30$ days prior to registration or planned during protocol therapy (patients may have received prochloperazine and other phenothiazines as prior antiemetic therapy). Not have concurrent use of ethyol. Not have severe cognitive compromise. History of CNS disease (e.g. brain metastases, seizure disorder). Concurrent use of amifostine, concurrent abdominal radiotherapy; concurrent use of quinolone antibiotic therapy. Chronic alcoholism (as determined by the investigator). Known hypersensitivity to olanzapine. Known cardiac arrhythmia, uncontrolled congestive heart failure or acute myocardial infarction within the previous six months. History of uncontrolled diabetes mellitus (stable insulin dose and/ or stable oral hypoglycaemic agent permitted). Planned chemotherapy or radiation during the 7 days following study initiation.

Baseline characteristics: not stated

Setting and location: not stated

Number of people screened: not stated

Number of participants randomised - all: not stated

Number randomised to each group:not stated

Number receiving treatment as allocated: not stated

Number not receiving treatment as allocated: not stated

Number dropped out: not stated

(no follow-up data for any outcome available)

Number excluded from analysis (for all outcomes): not stated

Number completed: not stated

Interventions

Intervention group $\mathbf{A}(\mathrm{N}=$ ?): $5 \mathrm{mg}$ olanzapine orally for 1-7 days daily

Control group $\mathbf{B}(\mathrm{N}=$ ?): Placebo orally for $1-7$ days daily

Use of additional treatments if any (co-interventions, additional analgesia): not stated

Outcomes

Primary outcomes

- Effect of olanzapine (time frame: nausea score from the VAS will be recorded each day daily for 7 days). Daily nausea scores (the primary objective) on days 1-7 of treatment for each participant from Groups 1 and 2 will be measured using the VAS rankings from $0-10$ where 0 is no nausea and 10 is the maximum nausea experienced by a Participant. Participants will be asked to record the average nausea score for each day in a diary and a study nurse will call the participant at the same time each day to remind them to record the nausea score and to inquire about any toxicities. The difference in the nausea scores for the participants in each group (Group A and Group B) will be compared

\section{Secondary outcomes}

- Effect of olanzapine (time frame: number of emetic episodes for each participant on each day of the 7-day treatment). The number of emetic episodes (a secondary outcome) by each participant in each Group ( $A$ and $B$ ) on each day of treatment will be recorded by each participant on each day of treatment in a diary. A study nurse will contact each participant at the same time of each day of the study to ask them to record the number of emetic episodes and report any toxicities. The number of emetic episodes for the participants in each Group for each $d$ of the treatments will be compared

- Number of participants with treatment-related AEs as assessed by CTCAE v 4.0". (time frame: daily assessment for 7 days for each participant in Groups A and A). AEs will be measured by patient-reported outcomes questionnaires and the Common Terminology Criteria for Adverse Events (CTCAE v4.0). A study nurse will contact each participant each day of the 7 days of treatment to inquire about any toxicities, specifically sedation and appetite. Sedation and appetite will be reported 
NCT03137121 (Continued)

by the participant in each Group on a VAS of $0-10$ with 0 being no sedation or no appetite to 10 indicating maximum sedation or maximum appetite

\begin{tabular}{ll}
\hline Starting date & April 2017 \\
\hline Contact information & rmnavari@gmail.com or rnavari@uab.edu \\
\hline
\end{tabular}

\section{Notes}

AE: adverse effects; CINV: chemotherapy-induced nausea and vomiting; CNS: central nervous system; CR: complete response; ECOG: Eastern Cooperative Oncology Group; HEC: highly emetogenic chemotherapy; IV: intravenous; MEC: moderately emetogenic chemotherapy; NRS: numeric rating scale; QOL: quality of life; ULN: upper limit of normal; VAS: visual analogue scale

\section{DATA AND ANALYSES}

\section{Comparison 1. Olanzapine vs placebo/no treatment}

\begin{tabular}{|c|c|c|c|c|}
\hline Outcome or subgroup title & No. of studies & $\begin{array}{l}\text { No. of partici- } \\
\text { pants }\end{array}$ & Statistical method & Effect size \\
\hline $\begin{array}{l}1 \text { No nausea or vomiting over trial peri- } \\
\text { od }\end{array}$ & 3 & 561 & $\begin{array}{l}\text { Risk Ratio (M-H, Random, } \\
95 \% \mathrm{CI})\end{array}$ & $1.98[1.59,2.47]$ \\
\hline 2 Serious adverse events & 7 & 889 & $\begin{array}{l}\text { Risk Ratio (M-H, Random, } \\
95 \% \mathrm{Cl})\end{array}$ & $2.46[0.48,12.55]$ \\
\hline $\begin{array}{l}3 \text { Participant preference - wish to use } \\
\text { drug in next treatment }\end{array}$ & 1 & 48 & $\begin{array}{l}\text { Risk Ratio (M-H, Fixed, 95\% } \\
\mathrm{Cl} \text { ) }\end{array}$ & $1.43[0.97,2.09]$ \\
\hline 4 Other adverse events & 4 & 332 & $\begin{array}{l}\text { Risk Ratio (M-H, Fixed, 95\% } \\
\mathrm{Cl} \text { ) }\end{array}$ & $1.71[0.99,2.96]$ \\
\hline 5 Somnolence/fatigue & 5 & 464 & $\begin{array}{l}\text { Risk Ratio (M-H, Fixed, 95\% } \\
\mathrm{Cl})\end{array}$ & $2.33[1.30,4.18]$ \\
\hline 6 Withdrawals due to all causes & 8 & 943 & $\begin{array}{l}\text { Risk Ratio (M-H, Fixed, 95\% } \\
\mathrm{Cl})\end{array}$ & $0.99[0.57,1.73]$ \\
\hline 7 Withdrawals due to lack of efficacy & 6 & 422 & $\begin{array}{l}\text { Risk Ratio (M-H, Fixed, 95\% } \\
\mathrm{Cl} \text { ) }\end{array}$ & $0.0[0.0,0.0]$ \\
\hline 8 Withdrawals due to adverse events & 6 & 422 & $\begin{array}{l}\text { Risk Ratio (M-H, Fixed, 95\% } \\
\mathrm{Cl})\end{array}$ & $3.0[0.13,70.16]$ \\
\hline $\begin{array}{l}9 \text { Breakthrough nausea/vomiting re- } \\
\text { quiring antiemetics over trial period }\end{array}$ & 2 & 501 & $\begin{array}{l}\text { Risk Ratio (M-H, Random, } \\
95 \% \mathrm{Cl})\end{array}$ & $0.38[0.10,1.47]$ \\
\hline 10 No nausea over trial period & 1 & 401 & $\begin{array}{l}\text { Risk Ratio (M-H, Fixed, 95\% } \\
\mathrm{Cl} \text { ) }\end{array}$ & $1.67[1.18,2.35]$ \\
\hline 11 No vomiting over trial period & 2 & 220 & $\begin{array}{l}\text { Risk Ratio (M-H, Fixed, 95\% } \\
\mathrm{Cl} \text { ) }\end{array}$ & $1.30[1.12,1.51]$ \\
\hline
\end{tabular}




\begin{tabular}{lllll}
\hline Outcome or subgroup title & No. of studies & $\begin{array}{l}\text { No. of partici- } \\
\text { pants }\end{array}$ & Statistical method & Effect size \\
\hline $\begin{array}{l}12 \text { No acute nausea (within 24 h of } \\
\text { chemotherapy) }\end{array}$ & 3 & 585 & $\begin{array}{l}\text { Risk Ratio (M-H, Random, } \\
95 \% \text { Cl) }\end{array}$ & 1.37 [0.95, 1.98] \\
\hline $\begin{array}{l}13 \text { No acute vomiting (within 24 h of } \\
\text { chemotherapy) }\end{array}$ & 5 & 702 & $\begin{array}{l}\text { Risk Ratio (M-H, Random, } \\
95 \% \text { Cl) }\end{array}$ & 1.11 [0.97, 1.28] \\
\hline $\begin{array}{l}14 \text { No acute nausea or vomiting (within } \\
24 \text { h of chemotherapy) }\end{array}$ & 2 & 164 & $\begin{array}{l}\text { Risk Ratio (M-H, Random, } \\
95 \% \text { Cl) }\end{array}$ & 1.21 [0.86, 1.69] \\
\hline $\begin{array}{l}15 \text { No delayed nausea (1-5 days after } \\
\text { chemotherapy) }\end{array}$ & 3 & 585 & $\begin{array}{l}\text { Risk Ratio (M-H, Fixed, 95\% } \\
\text { Cl) }\end{array}$ & $1.71[1.40,2.09]$ \\
\hline $\begin{array}{l}16 \text { No delayed vomiting (1-5 days after } \\
\text { chemotherapy) }\end{array}$ & 5 & 702 & $\begin{array}{l}\text { Risk Ratio (M-H, Fixed, 95\% } \\
\text { Cl) }\end{array}$ & 1.28 [1.14, 1.42] \\
\hline $\begin{array}{l}17 \text { No delayed nausea or vomiting (1-5 } \\
\text { days after chemotherapy) }\end{array}$ & 3 & 264 & $\begin{array}{l}\text { Risk Ratio (M-H, Fixed, 95\% } \\
\text { Cl) }\end{array}$ & 1.98 [1.61, 2.44] \\
\hline
\end{tabular}

Analysis 1.1. Comparison 1 Olanzapine vs placebo/no treatment, Outcome 1 No nausea or vomiting over trial period.

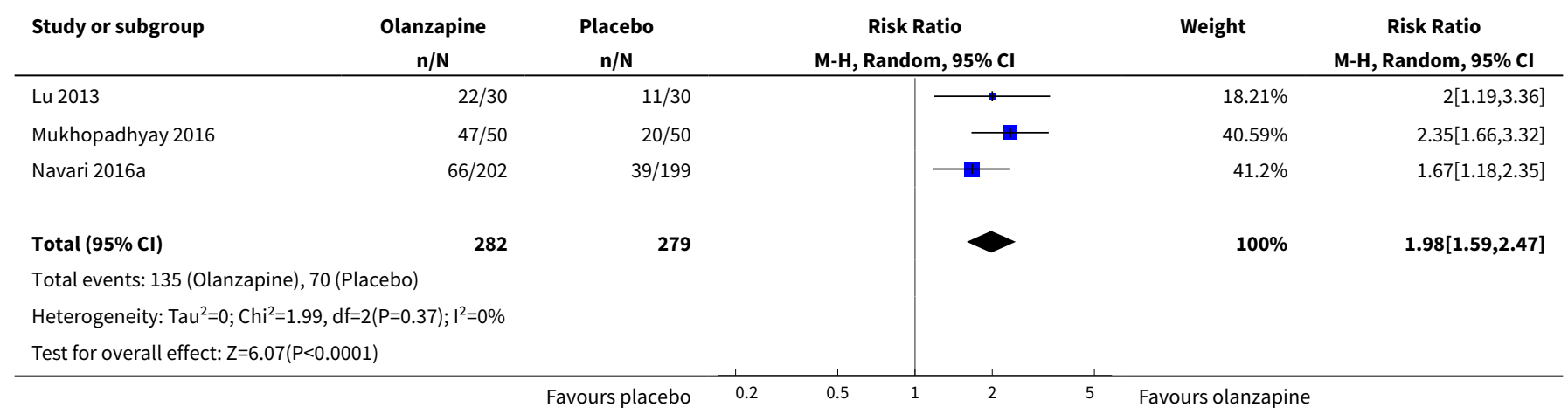

Analysis 1.2. Comparison 1 Olanzapine vs placebo/no treatment, Outcome 2 Serious adverse events.

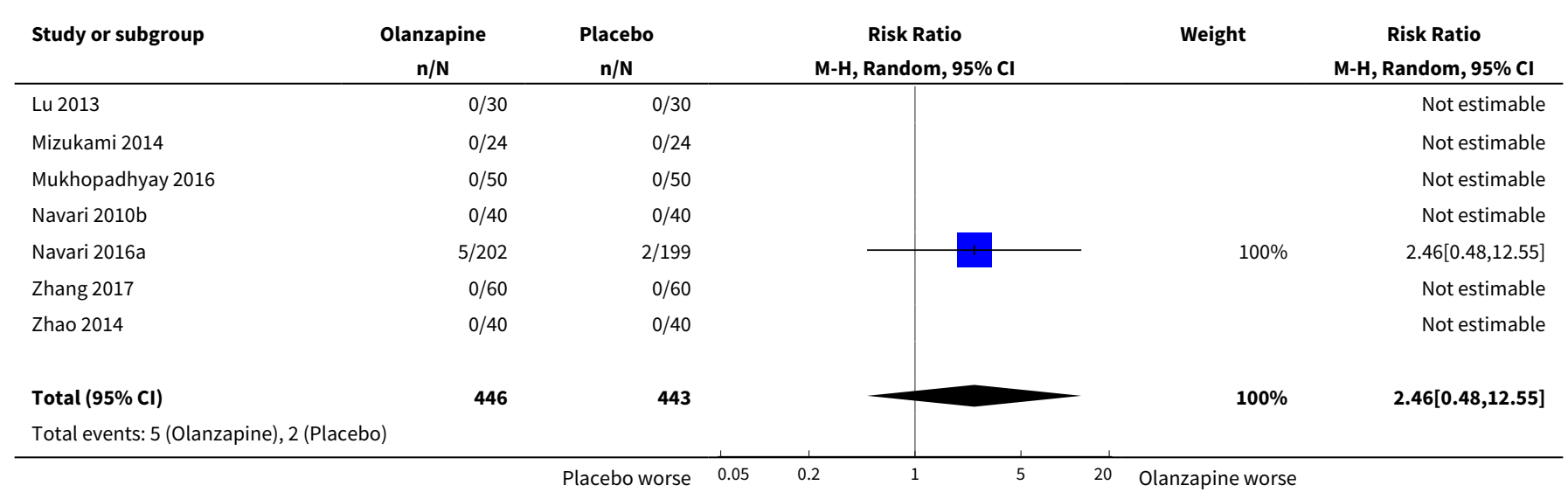




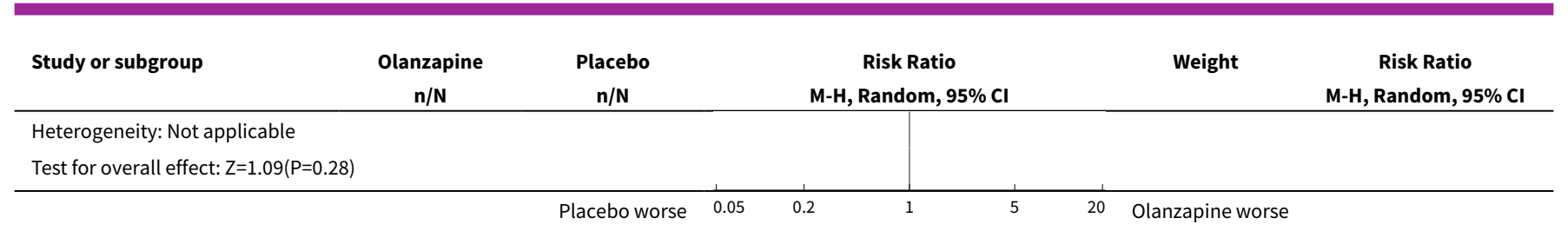

Analysis 1.3. Comparison 1 Olanzapine vs placebo/no treatment, Outcome 3 Participant preference - wish to use drug in next treatment.

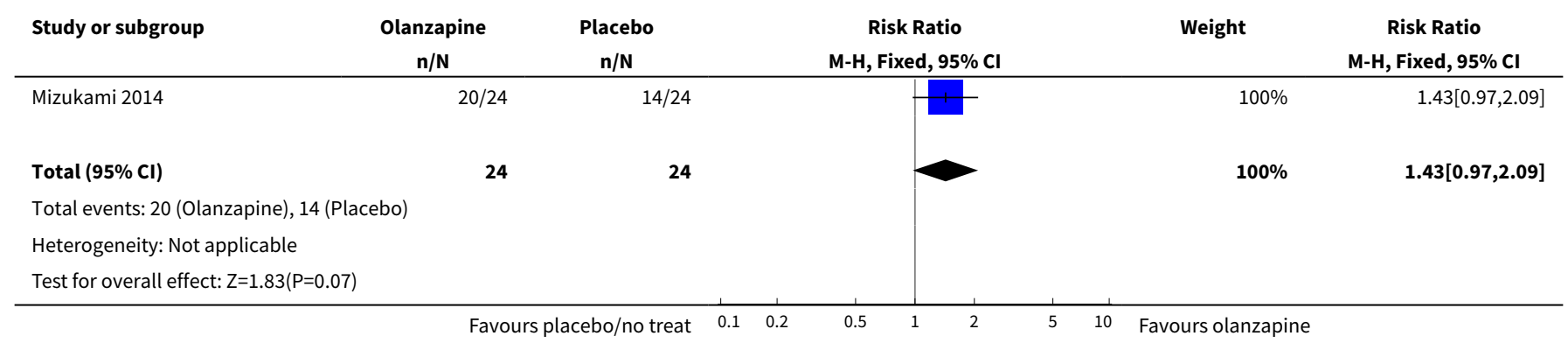

Analysis 1.4. Comparison 1 Olanzapine vs placebo/no treatment, Outcome 4 Other adverse events.

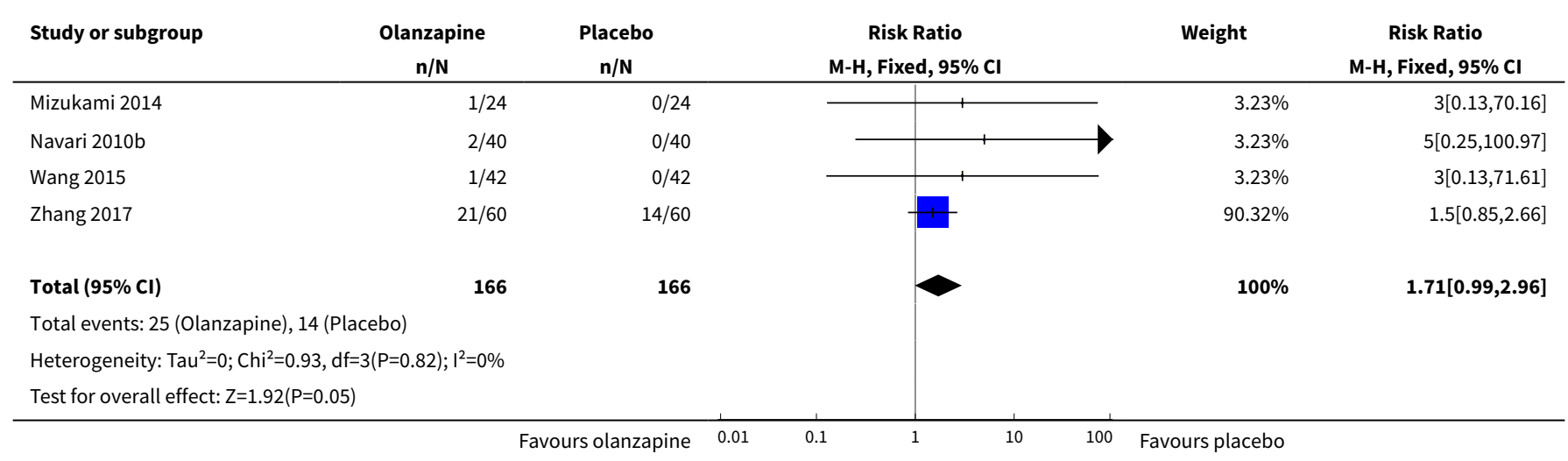

Analysis 1.5. Comparison 1 Olanzapine vs placebo/no treatment, Outcome 5 Somnolence/fatigue.

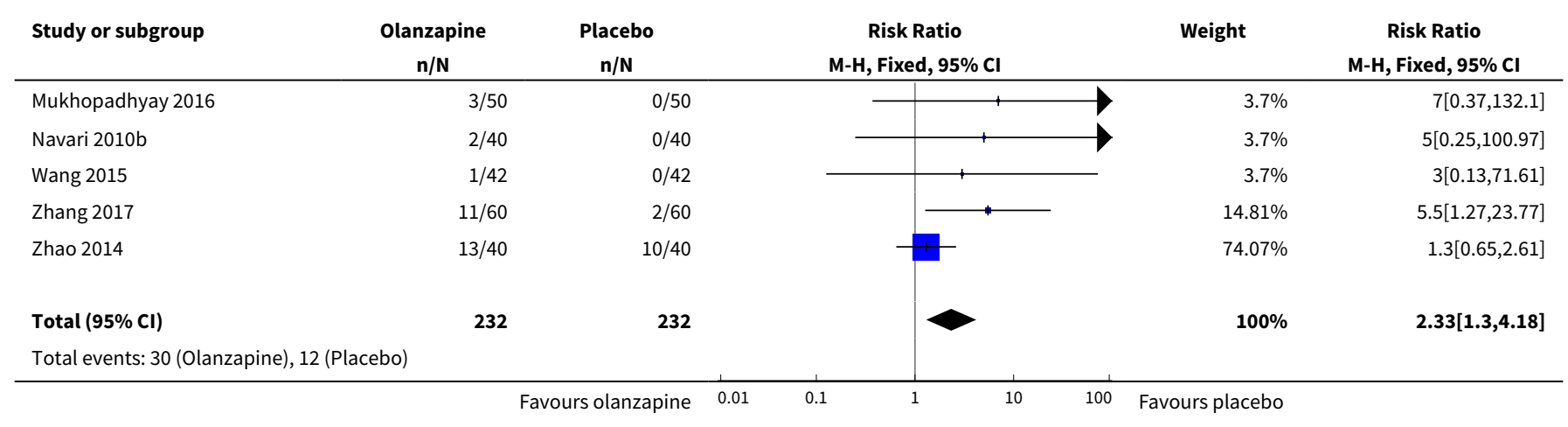




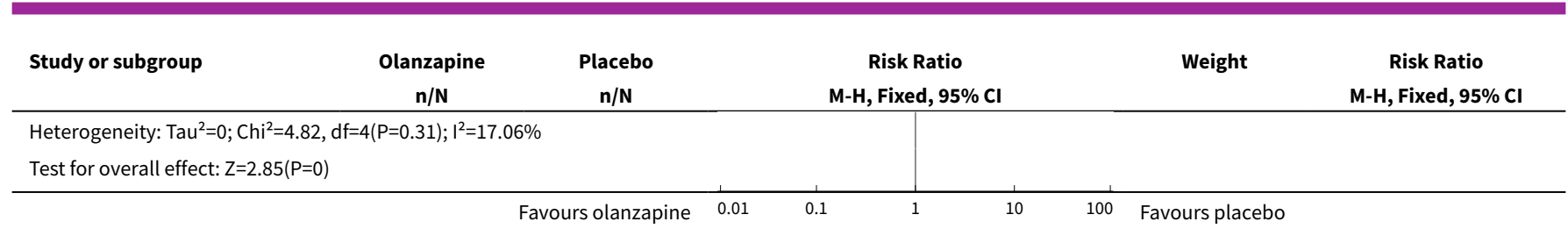

Analysis 1.6. Comparison 1 Olanzapine vs placebo/no treatment, Outcome 6 Withdrawals due to all causes.

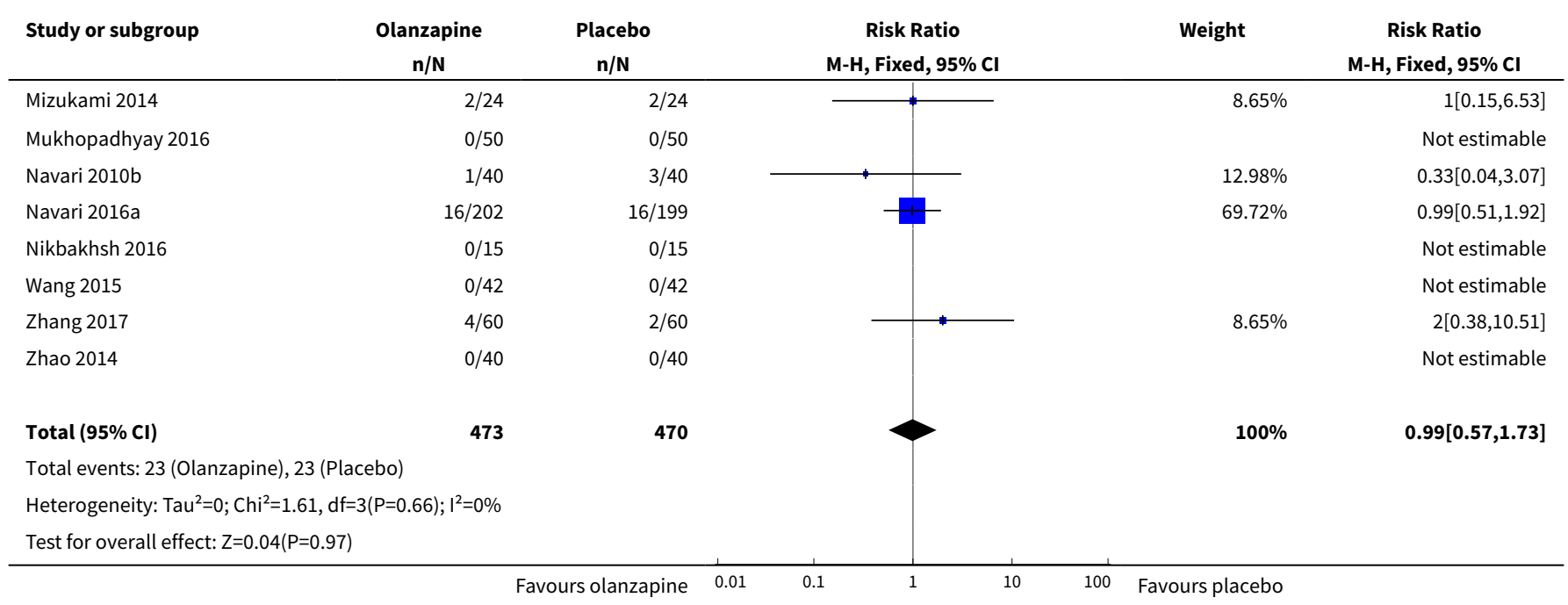

Analysis 1.7. Comparison 1 Olanzapine vs placebo/no treatment, Outcome 7 Withdrawals due to lack of efficacy.

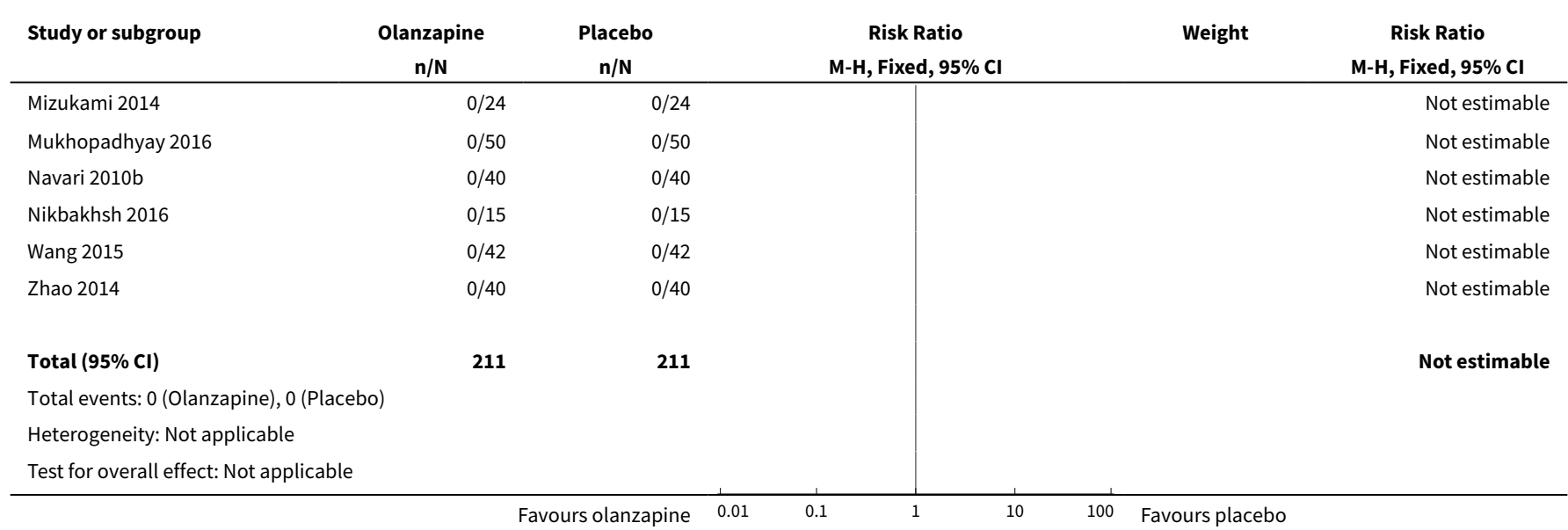


Analysis 1.8. Comparison 1 Olanzapine vs placebo/no treatment, Outcome 8 Withdrawals due to adverse events.

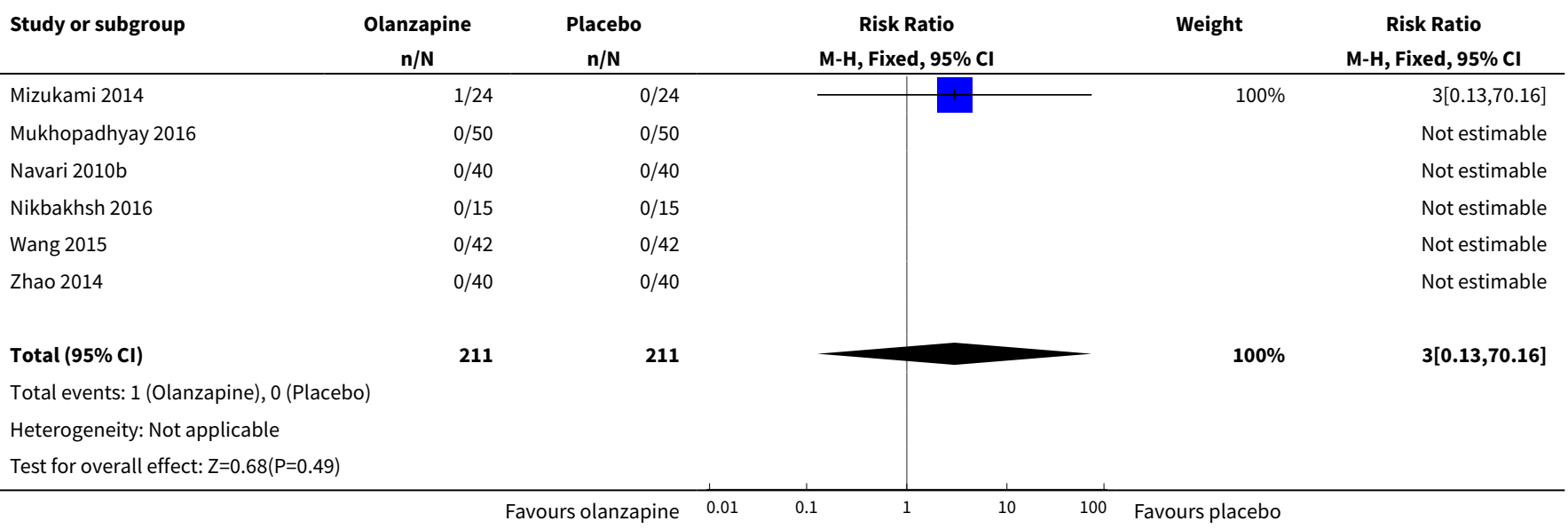

Analysis 1.9. Comparison 1 Olanzapine vs placebo/no treatment, Outcome 9 Breakthrough nausea/vomiting requiring antiemetics over trial period.

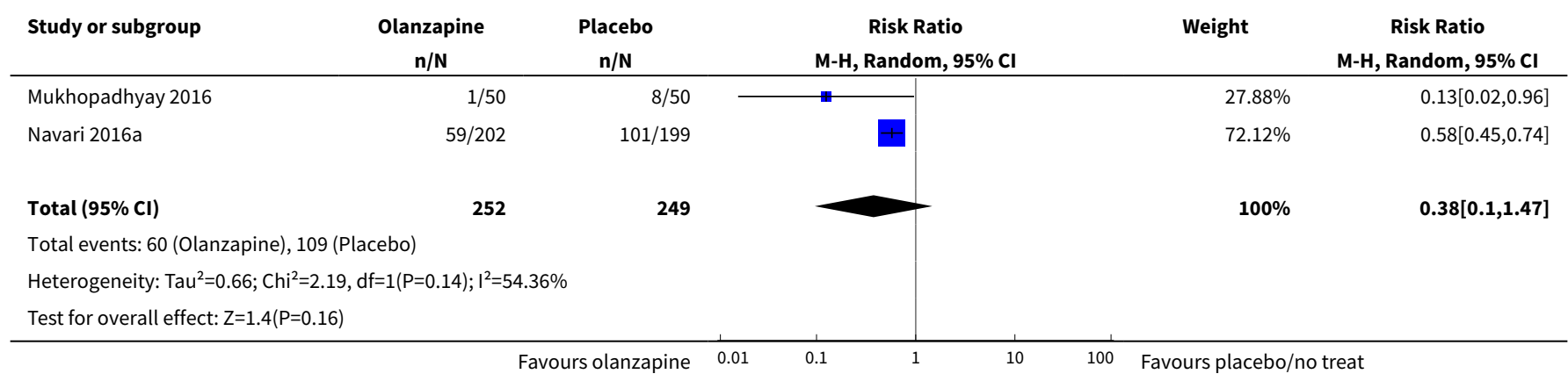

\section{Analysis 1.10. Comparison 1 Olanzapine vs placebo/no treatment, Outcome 10 No nausea over trial period.}

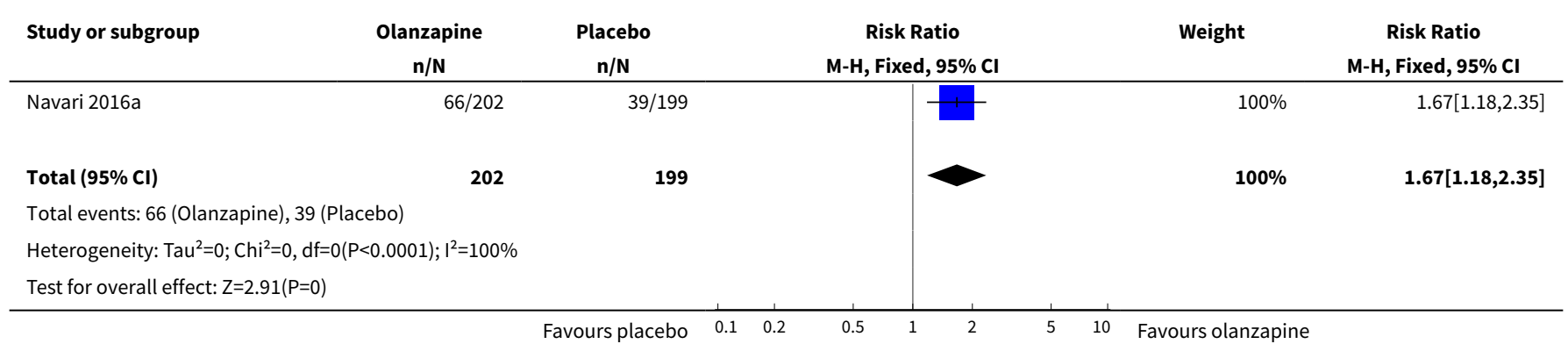


Analysis 1.11. Comparison 1 Olanzapine vs placebo/no treatment, Outcome 11 No vomiting over trial period.

\begin{tabular}{|c|c|c|c|c|c|}
\hline Study or subgroup & $\begin{array}{c}\text { Olanzapine } \\
\mathbf{n} / \mathbf{N}\end{array}$ & $\begin{array}{c}\text { Placebo } \\
\mathbf{n} / \mathbf{N}\end{array}$ & $\begin{array}{c}\text { Risk Ratio } \\
\text { M-H, Fixed, 95\% Cl }\end{array}$ & Weight & $\begin{array}{c}\text { Risk Ratio } \\
\text { M-H, Fixed, } 95 \% \mathrm{Cl} \\
\end{array}$ \\
\hline Mukhopadhyay 2016 & $48 / 50$ & $37 / 50$ & - & $50.68 \%$ & $1.3[1.09,1.54]$ \\
\hline Zhang 2017 & $47 / 60$ & $36 / 60$ & -1 & $49.32 \%$ & $1.31[1.02,1.67]$ \\
\hline Total $(95 \% \mathrm{Cl})$ & 110 & 110 & $V$ & $100 \%$ & $1.3[1.12,1.51]$ \\
\hline \multicolumn{6}{|c|}{ Total events: 95 (Olanzapine), 73 (Placebo) } \\
\hline Test for overall effect: & & & & & \\
\hline
\end{tabular}

Analysis 1.12. Comparison 1 Olanzapine vs placebo/no treatment, Outcome 12 No acute nausea (within $24 \mathrm{~h}$ of chemotherapy).

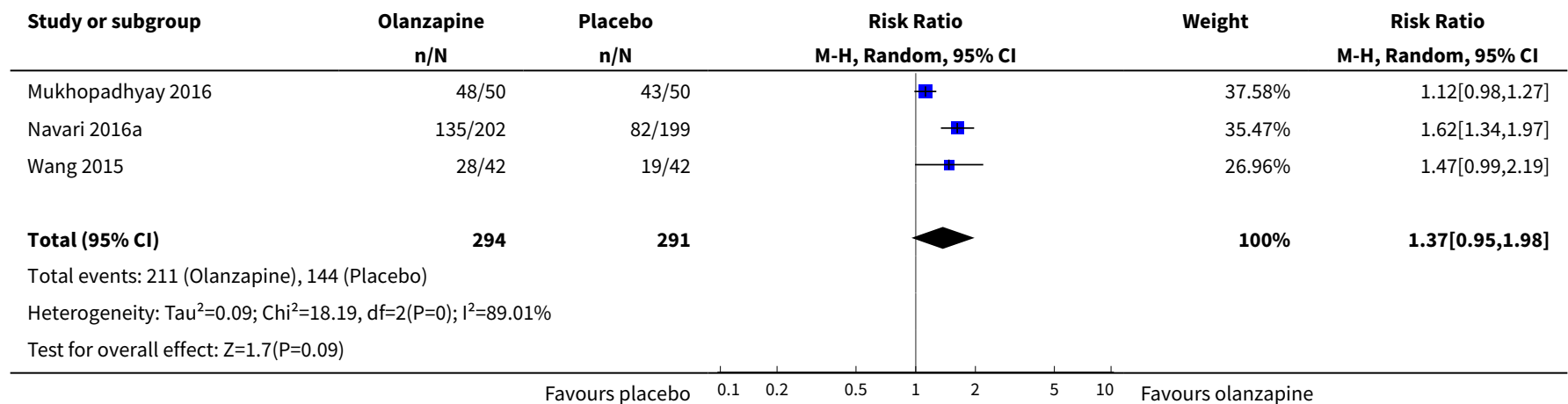

Analysis 1.13. Comparison 1 Olanzapine vs placebo/no treatment, Outcome 13 No acute vomiting (within $24 \mathrm{~h}$ of chemotherapy).

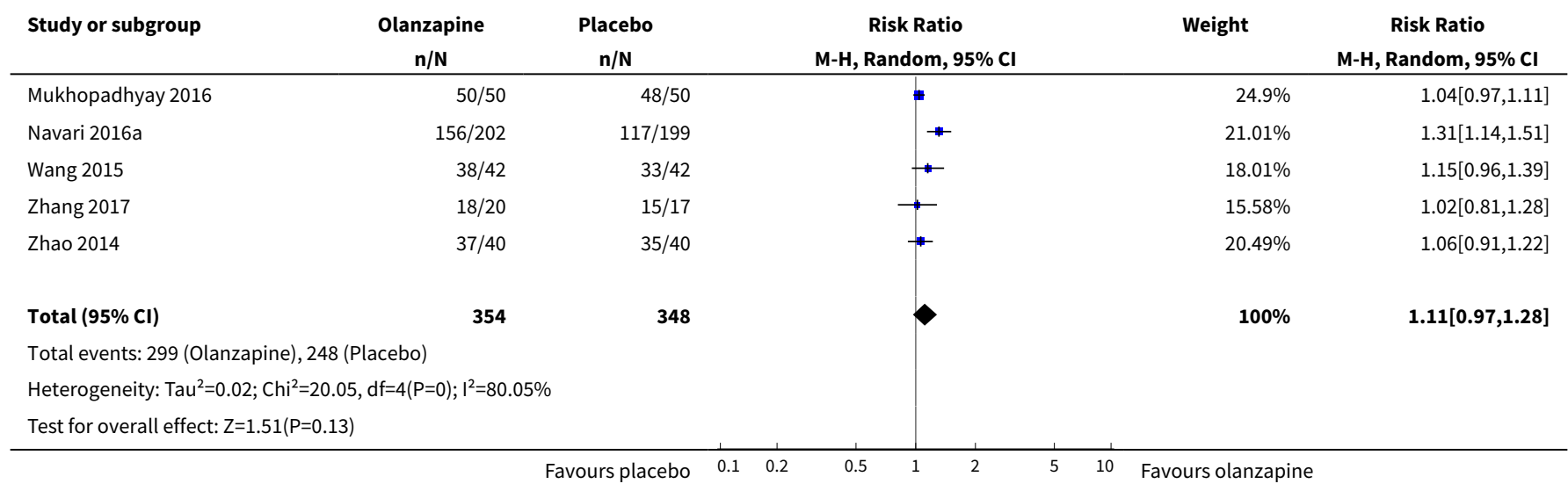


Analysis 1.14. Comparison 1 Olanzapine vs placebo/no treatment, Outcome $14 \mathrm{No}$ acute nausea or vomiting (within $24 \mathrm{~h}$ of chemotherapy).

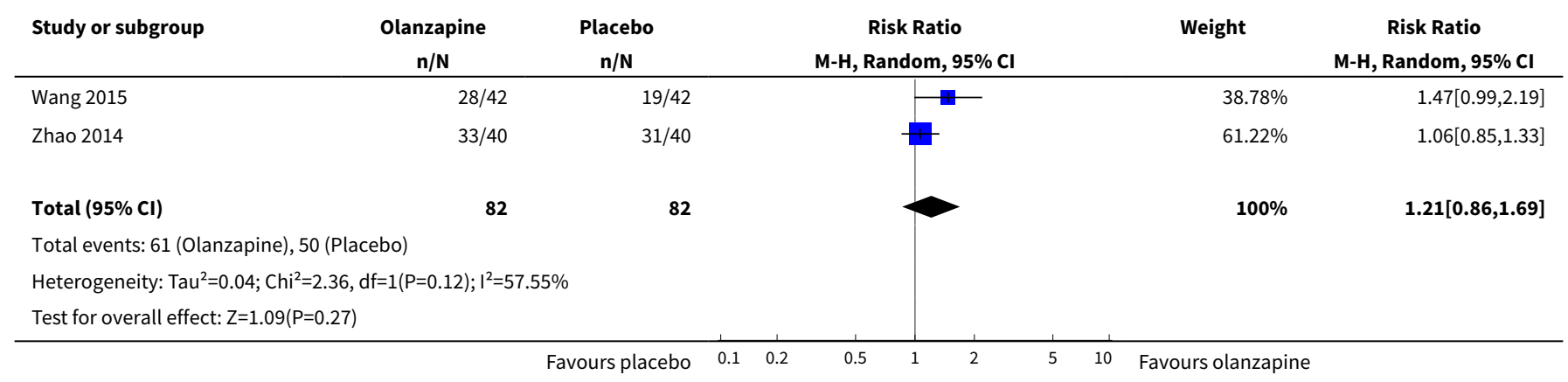

Analysis 1.15. Comparison 1 Olanzapine vs placebo/no treatment, Outcome 15 No delayed nausea (1-5 days after chemotherapy).

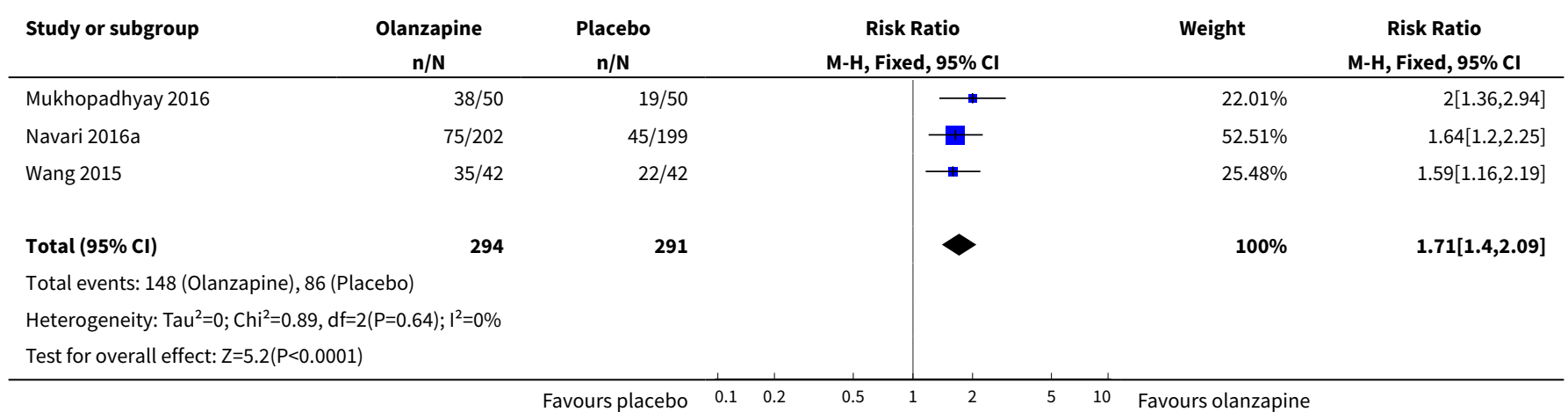

Analysis 1.16. Comparison 1 Olanzapine vs placebo/no treatment, Outcome 16 No delayed vomiting (1-5 days after chemotherapy).

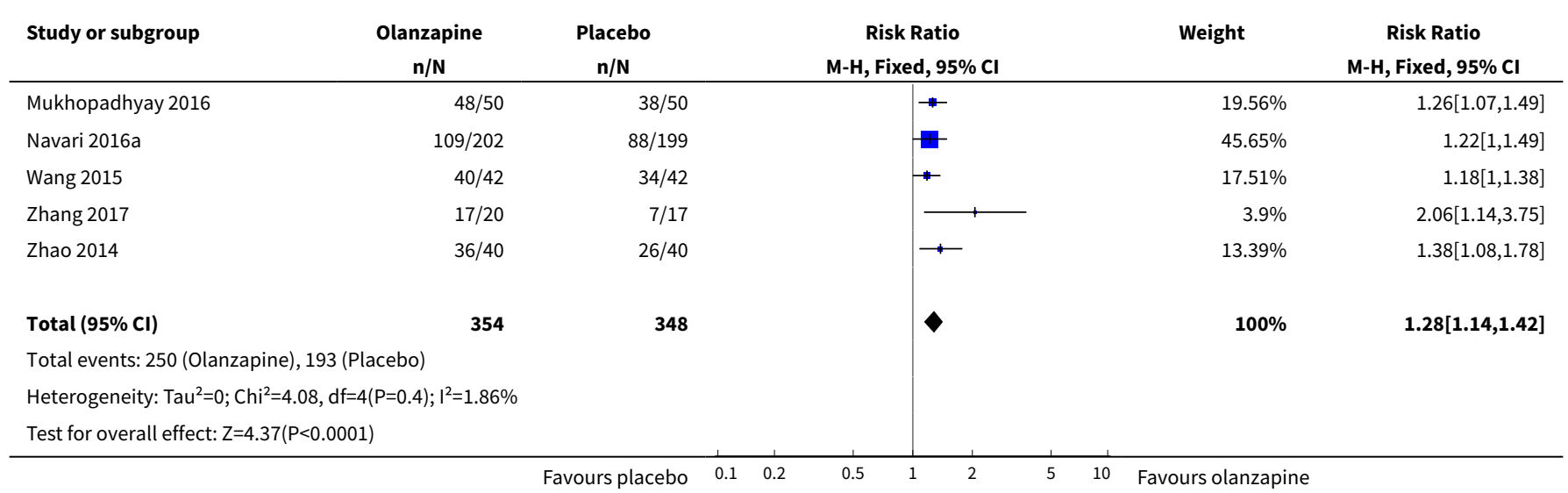


Analysis 1.17. Comparison 1 Olanzapine vs placebo/no treatment, Outcome 17 No delayed nausea or vomiting (1-5 days after chemotherapy).

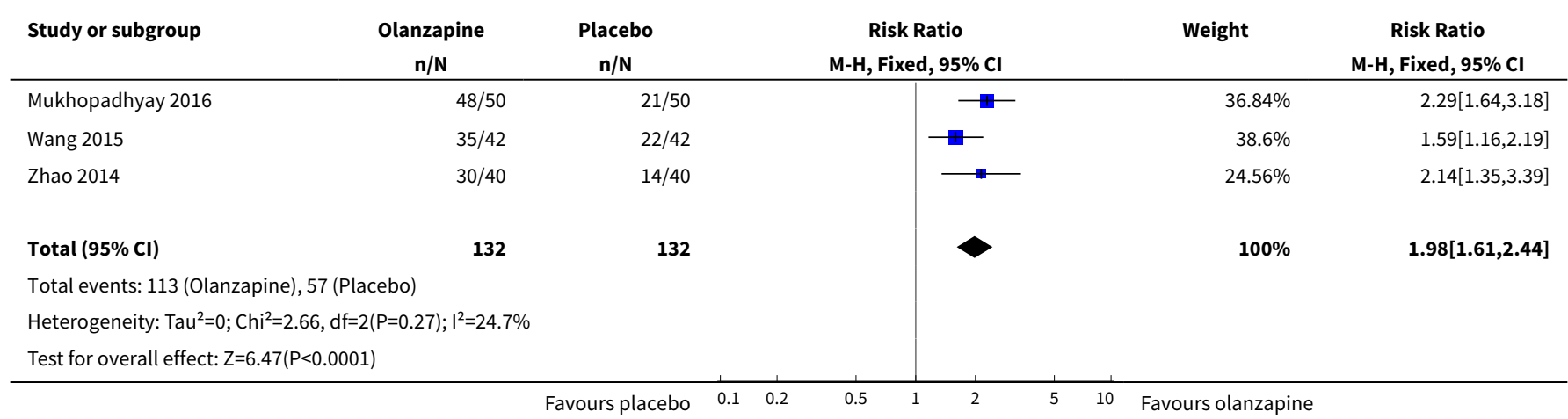

\section{Comparison 2. Olanzapine vs NK1 antagonist}

\begin{tabular}{|c|c|c|c|c|}
\hline Outcome or subgroup title & No. of studies & $\begin{array}{l}\text { No. of partici- } \\
\text { pants }\end{array}$ & Statistical method & Effect size \\
\hline 1 Serious adverse events & 1 & 20 & Risk Ratio (M-H, Fixed, 95\% Cl) & $0.33[0.02,7.32]$ \\
\hline 2 Somnolence/fatigue & 1 & 20 & Risk Ratio (M-H, Fixed, 95\% Cl) & $1.33[0.40,4.49]$ \\
\hline 3 Withdrawals due to all causes & 1 & 20 & Risk Ratio (M-H, Fixed, 95\% Cl) & $3.0[0.14,65.90]$ \\
\hline $\begin{array}{l}4 \text { Withdrawals due to lack of effi- } \\
\text { cacy }\end{array}$ & 1 & 20 & Risk Ratio (M-H, Fixed, 95\% Cl) & $0.0[0.0,0.0]$ \\
\hline $\begin{array}{l}5 \text { Withdrawals due to adverse } \\
\text { events }\end{array}$ & 1 & 20 & Risk Ratio (M-H, Fixed, 95\% Cl) & $0.0[0.0,0.0]$ \\
\hline 6 Withdrawals due to death & 1 & 20 & Risk Ratio (M-H, Fixed, 95\% Cl) & $0.0[0.0,0.0]$ \\
\hline 7 No nausea over trial period & 1 & 20 & Risk Ratio (M-H, Fixed, 95\% Cl) & $0.67[0.14,3.17]$ \\
\hline $\begin{array}{l}8 \text { No acute nausea (within } 24 \mathrm{~h} \text { of } \\
\text { chemotherapy) }\end{array}$ & 1 & 20 & Risk Ratio (M-H, Fixed, 95\% Cl) & $0.6[0.19,1.86]$ \\
\hline $\begin{array}{l}9 \text { No delayed nausea ( } 1-5 \text { days af- } \\
\text { ter chemotherapy) }\end{array}$ & 1 & 20 & Risk Ratio (M-H, Fixed, 95\% Cl) & $1.33[0.40,4.49]$ \\
\hline
\end{tabular}

Analysis 2.1. Comparison 2 Olanzapine vs NK1 antagonist, Outcome 1 Serious adverse events.

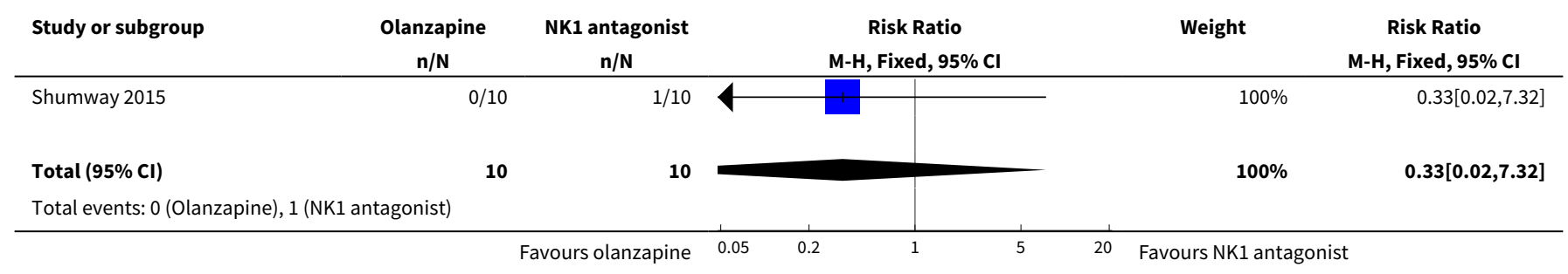




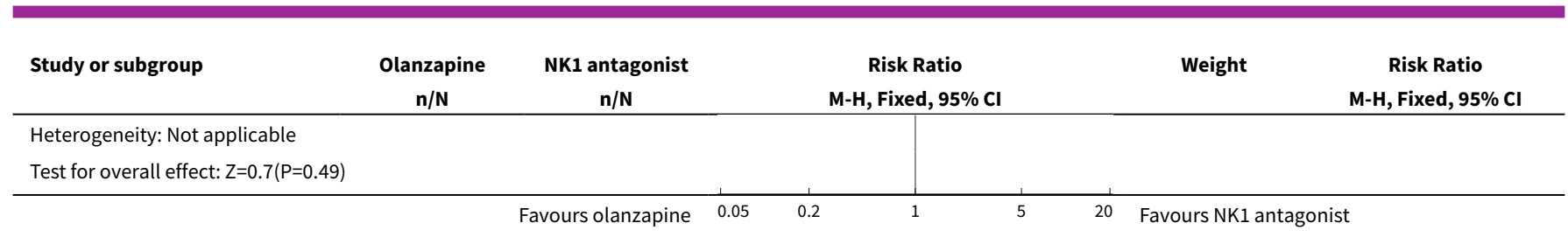

\section{Analysis 2.2. Comparison 2 Olanzapine vs NK1 antagonist, Outcome 2 Somnolence/fatigue.}

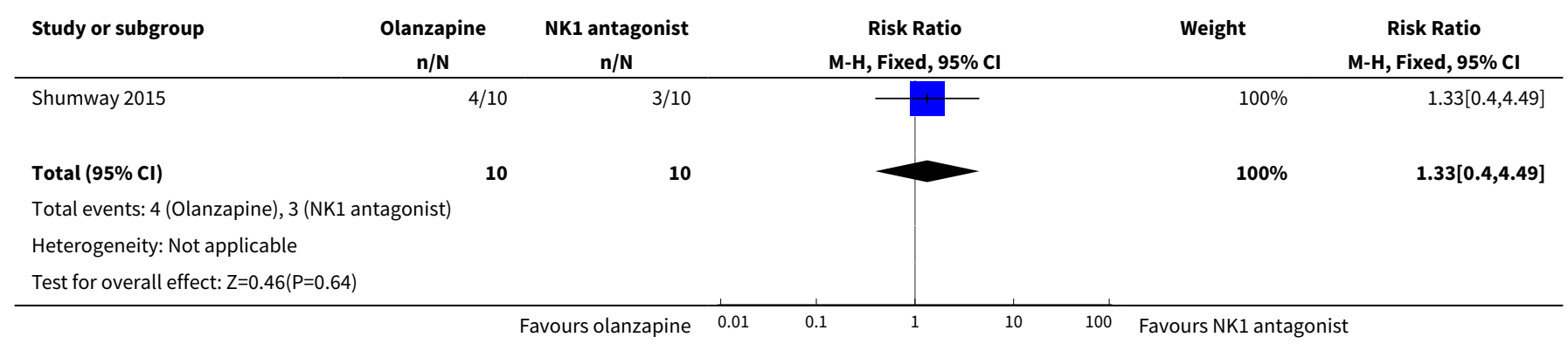

Analysis 2.3. Comparison 2 Olanzapine vs NK1 antagonist, Outcome 3 Withdrawals due to all causes.

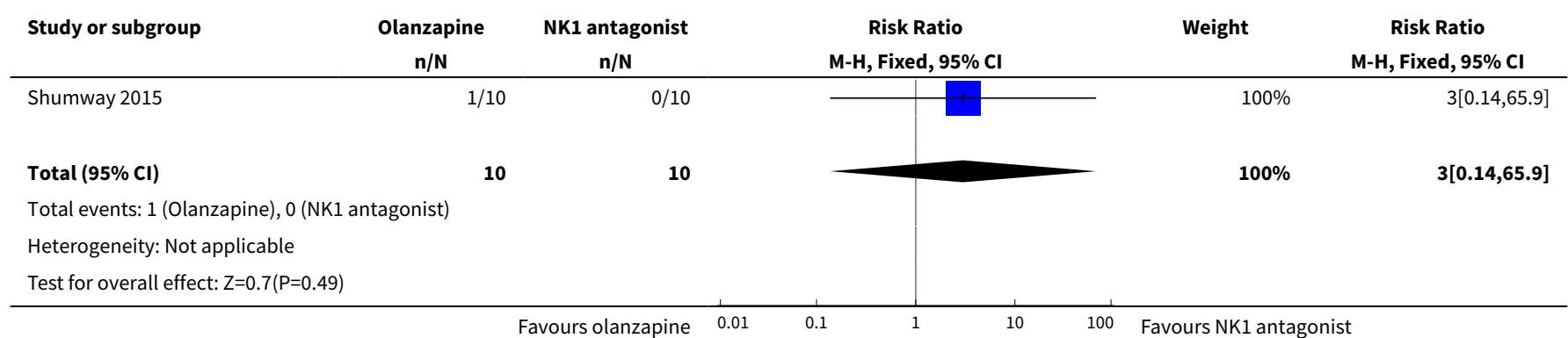

Analysis 2.4. Comparison 2 Olanzapine vs NK1 antagonist, Outcome 4 Withdrawals due to lack of efficacy.

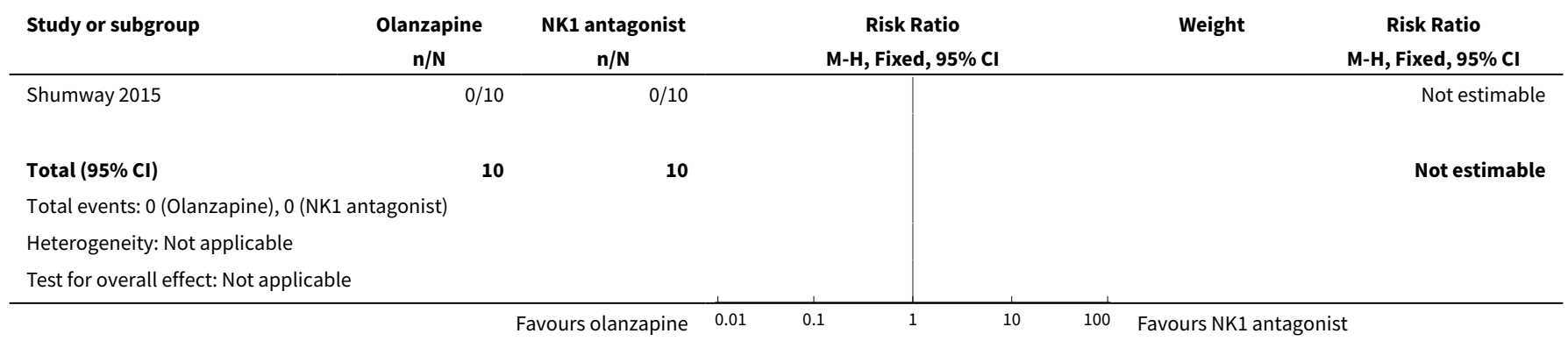


Analysis 2.5. Comparison 2 Olanzapine vs NK1 antagonist, Outcome 5 Withdrawals due to adverse events.

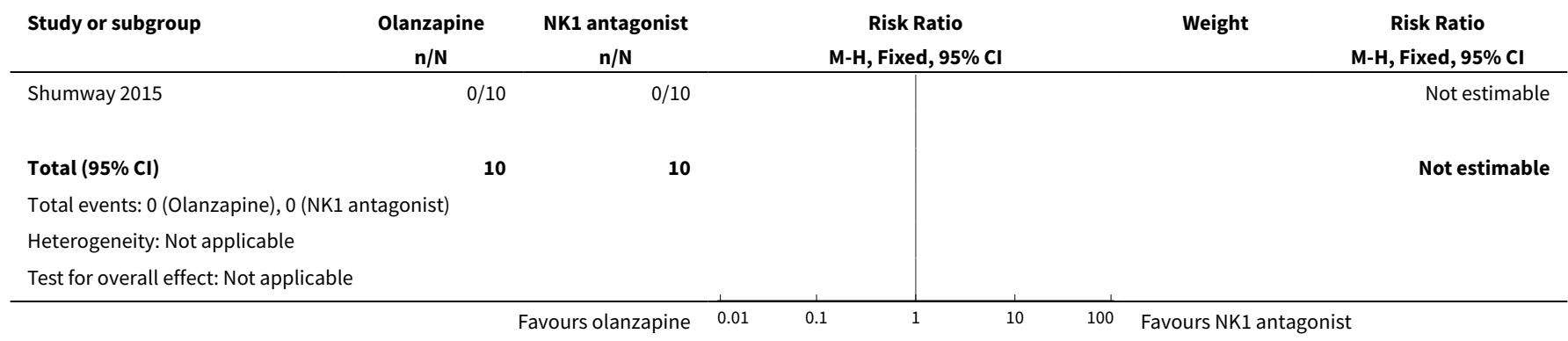

Analysis 2.6. Comparison 2 Olanzapine vs NK1 antagonist, Outcome 6 Withdrawals due to death.

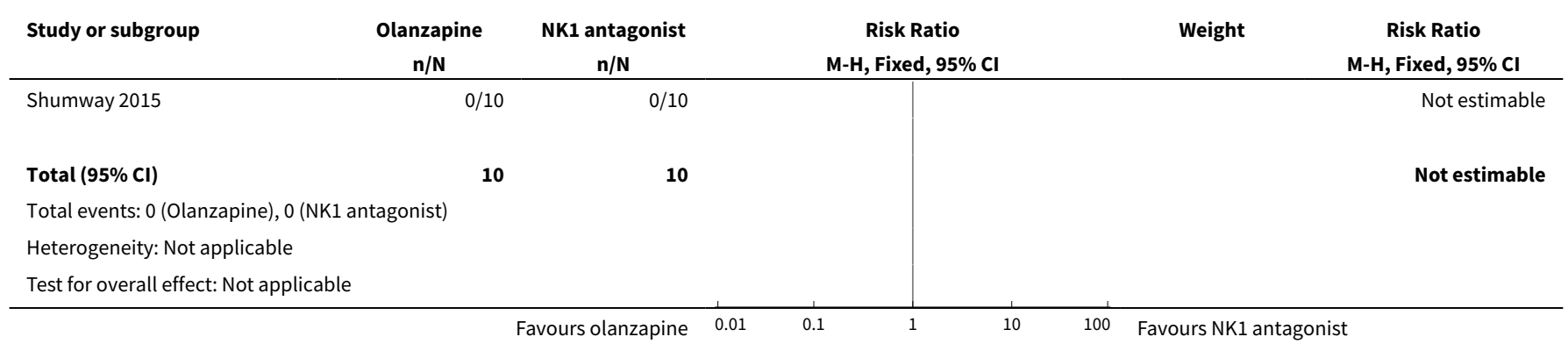

Analysis 2.7. Comparison 2 Olanzapine vs NK1 antagonist, Outcome 7 No nausea over trial period.

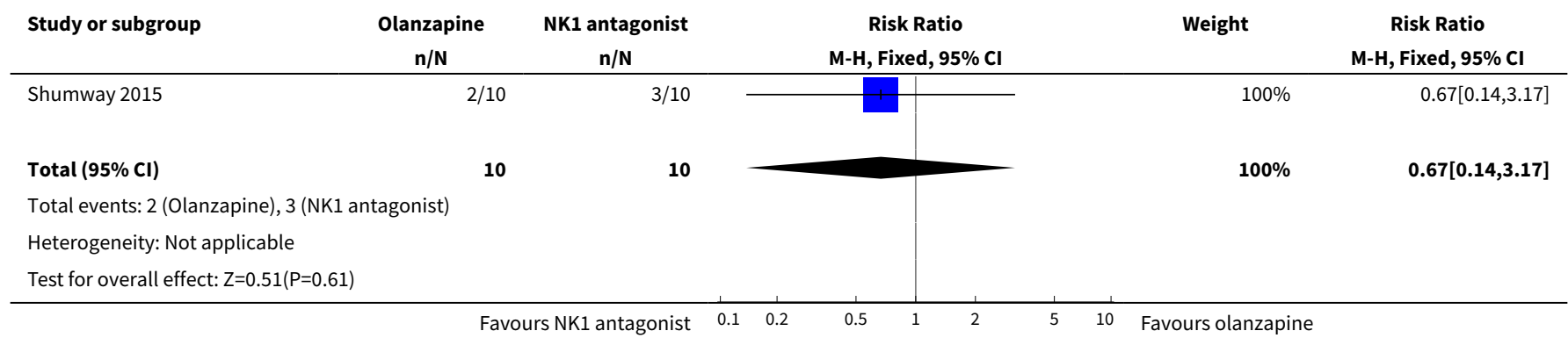

Analysis 2.8. Comparison 2 Olanzapine vs NK1 antagonist, Outcome 8 No acute nausea (within $24 \mathrm{~h}$ of chemotherapy).

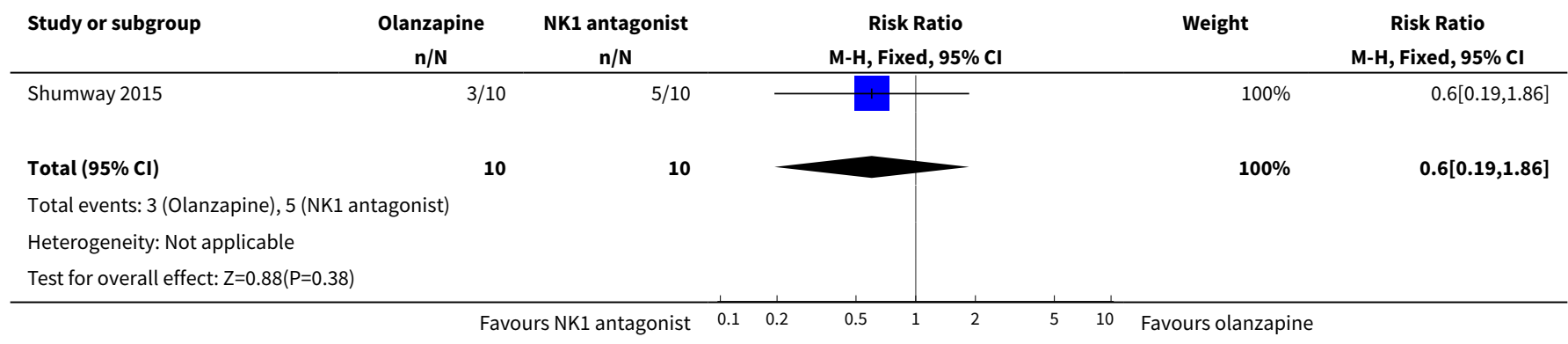


Analysis 2.9. Comparison 2 Olanzapine vs NK1 antagonist, Outcome 9 No delayed nausea (1-5 days after chemotherapy).

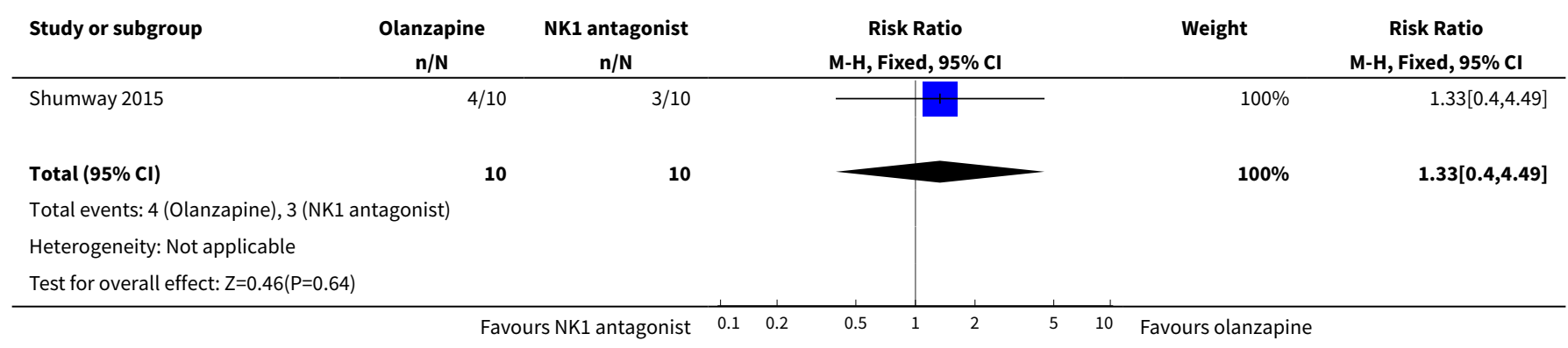

\section{Comparison 3. Olanzapine vs prokinetic}

\begin{tabular}{|c|c|c|c|c|}
\hline Outcome or subgroup title & No. of studies & $\begin{array}{l}\text { No. of partici- } \\
\text { pants }\end{array}$ & Statistical method & Effect size \\
\hline 1 Serious adverse events & 1 & 112 & Risk Ratio (M-H, Fixed, 95\% Cl) & $0.0[0.0,0.0]$ \\
\hline 2 Withdrawals due to all causes & 1 & 112 & Risk Ratio (M-H, Fixed, 95\% Cl) & $0.93[0.14,6.38]$ \\
\hline $\begin{array}{l}3 \text { Withdrawals due to lack of effi- } \\
\text { cacy }\end{array}$ & 1 & 112 & Risk Ratio (M-H, Fixed, 95\% Cl) & $0.47[0.04,4.99]$ \\
\hline $\begin{array}{l}4 \text { Withdrawals due to adverse } \\
\text { events }\end{array}$ & 1 & 112 & Risk Ratio (M-H, Fixed, 95\% Cl) & $0.0[0.0,0.0]$ \\
\hline 5 Withdrawals due to death & 1 & 112 & Risk Ratio (M-H, Fixed, 95\% Cl) & $0.0[0.0,0.0]$ \\
\hline 6 No nausea over trial period & 1 & 112 & Risk Ratio (M-H, Fixed, 95\% Cl) & $2.95[1.73,5.02]$ \\
\hline 7 No vomiting over trial period & 1 & 112 & Risk Ratio (M-H, Fixed, 95\% Cl) & $3.03[1.78,5.14]$ \\
\hline
\end{tabular}

Analysis 3.1. Comparison 3 Olanzapine vs prokinetic, Outcome 1 Serious adverse events.

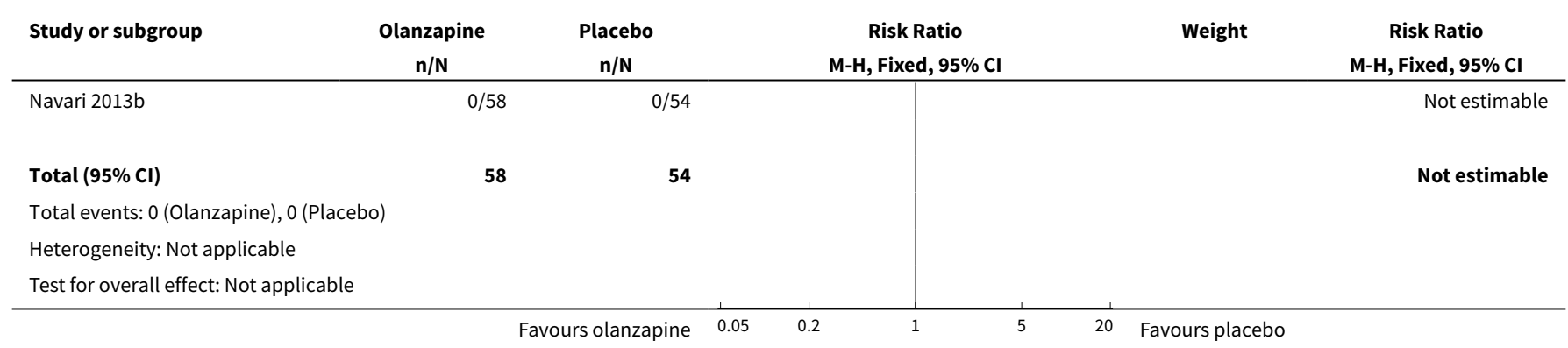


Analysis 3.2. Comparison 3 Olanzapine vs prokinetic, Outcome 2 Withdrawals due to all causes.

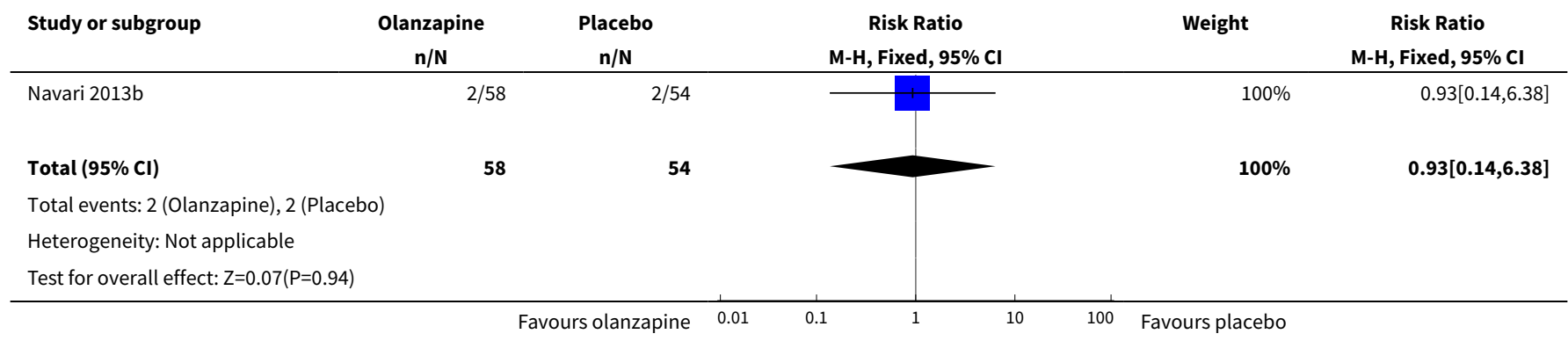

Analysis 3.3. Comparison 3 Olanzapine vs prokinetic, Outcome 3 Withdrawals due to lack of efficacy.

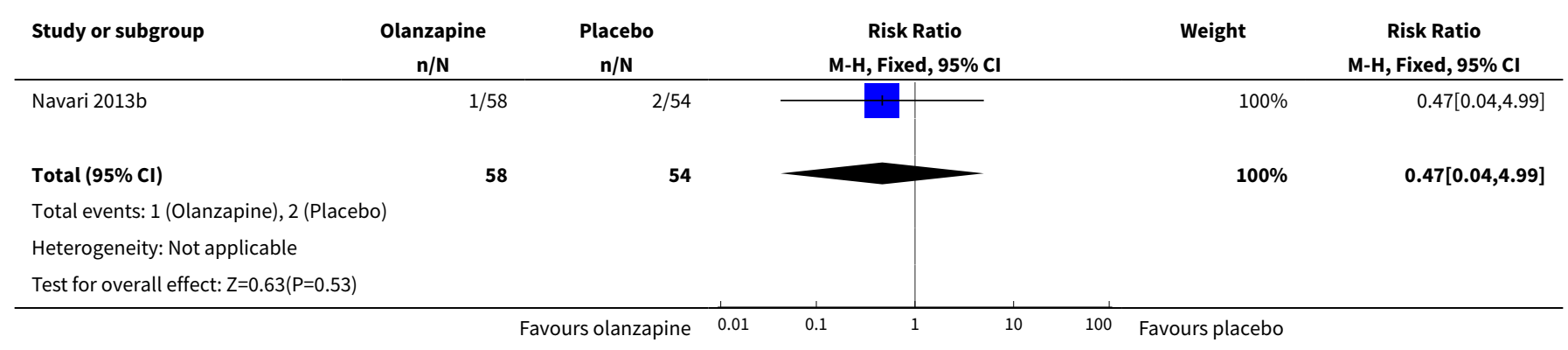

Analysis 3.4. Comparison 3 Olanzapine vs prokinetic, Outcome 4 Withdrawals due to adverse events.

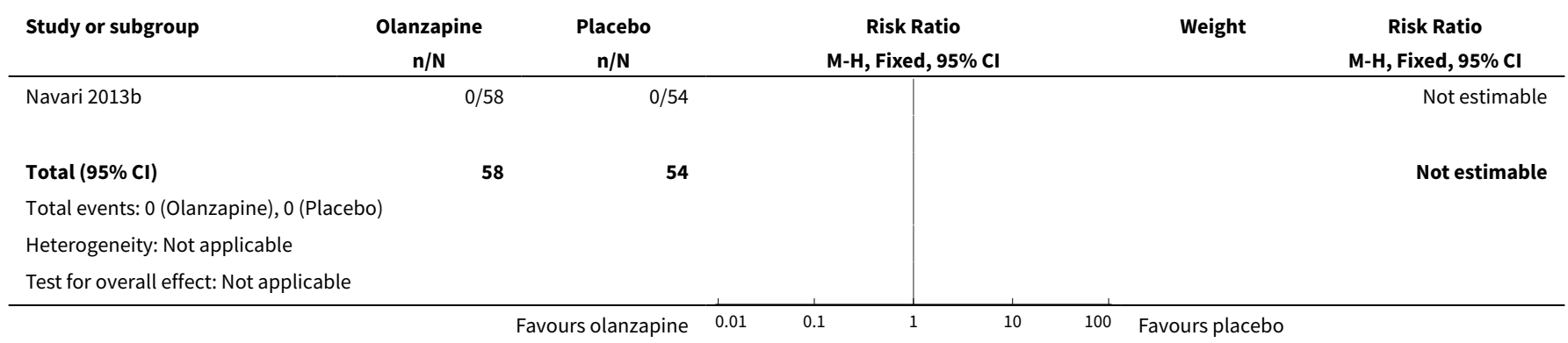

Analysis 3.5. Comparison 3 Olanzapine vs prokinetic, Outcome 5 Withdrawals due to death.

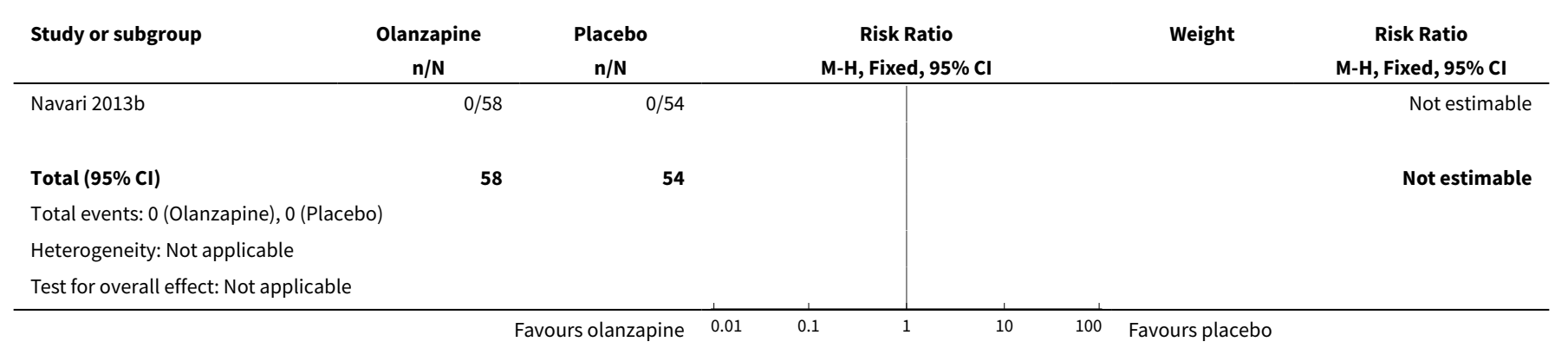


Analysis 3.6. Comparison 3 Olanzapine vs prokinetic, Outcome 6 No nausea over trial period.

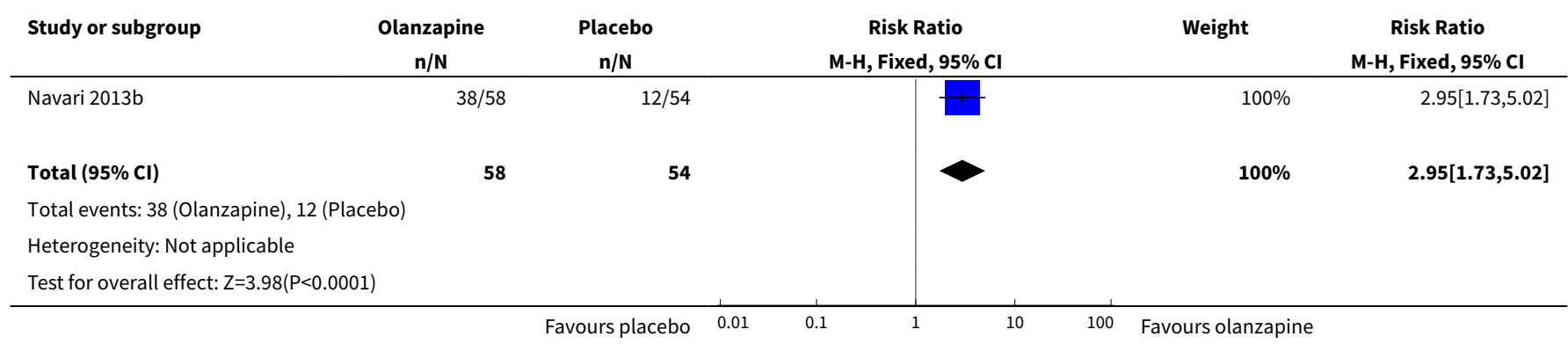

Analysis 3.7. Comparison 3 Olanzapine vs prokinetic, Outcome 7 No vomiting over trial period.

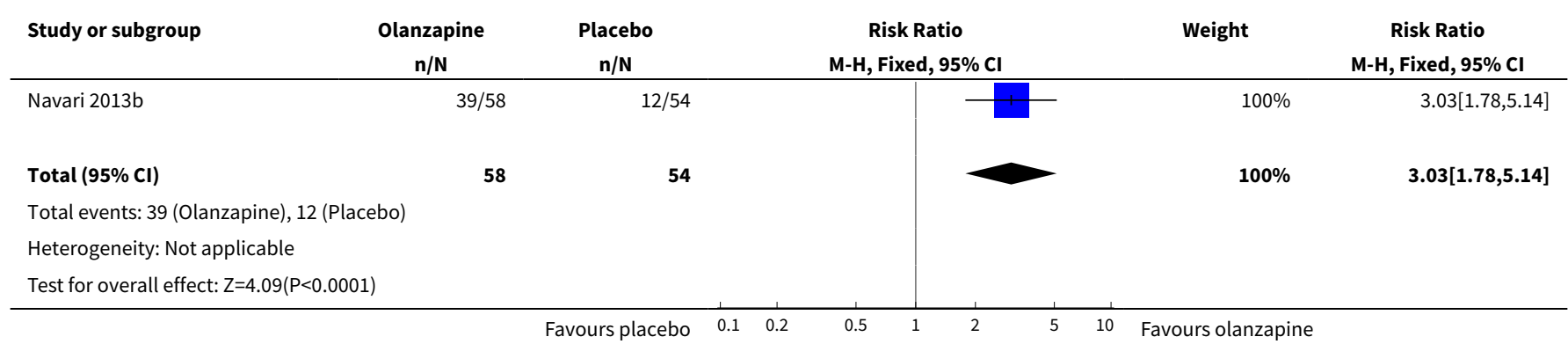

\section{Comparison 4. Olanzapine vs 5-HT3 antagonist}

\begin{tabular}{|c|c|c|c|c|}
\hline Outcome or subgroup title & No. of studies & $\begin{array}{l}\text { No. of partici- } \\
\text { pants }\end{array}$ & Statistical method & Effect size \\
\hline 1 Serious adverse events & 1 & 62 & Risk Ratio (M-H, Fixed, 95\% Cl) & $0.0[0.0,0.0]$ \\
\hline $\begin{array}{l}250 \% \text { improvement in nausea at } 24 \\
\text { h on a validated scale }\end{array}$ & 1 & 62 & Risk Ratio (M-H, Fixed, 95\% Cl) & $1.36[0.80,2.34]$ \\
\hline $\begin{array}{l}350 \% \text { improvement in nausea at } 48 \\
\text { h on a validated scale }\end{array}$ & 1 & 62 & Risk Ratio (M-H, Fixed, 95\% Cl) & $1.82[1.11,2.97]$ \\
\hline 4 Withdrawals due to all causes & 1 & 62 & Risk Ratio (M-H, Fixed, 95\% Cl) & $3.64[0.35,37.88]$ \\
\hline 5 Withdrawals due to lack of efficacy & 1 & 62 & Risk Ratio (M-H, Fixed, 95\% Cl) & $3.64[0.35,37.88]$ \\
\hline $\begin{array}{l}6 \text { Withdrawals due to adverse } \\
\text { events }\end{array}$ & 1 & 62 & Risk Ratio (M-H, Fixed, 95\% Cl) & $0.0[0.0,0.0]$ \\
\hline 7 Withdrawals due to death & 1 & 62 & Risk Ratio (M-H, Fixed, 95\% Cl) & $0.0[0.0,0.0]$ \\
\hline
\end{tabular}


Analysis 4.1. Comparison 4 Olanzapine vs 5-HT3 antagonist, Outcome 1 Serious adverse events.

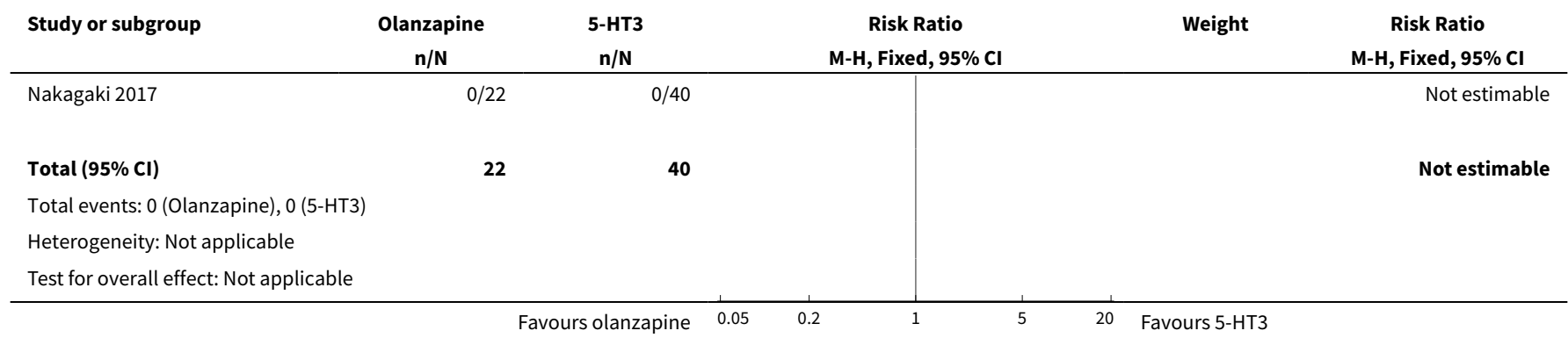

Analysis 4.2. Comparison 4 Olanzapine vs 5-HT3 antagonist, Outcome $250 \%$ improvement in nausea at $24 \mathrm{~h}$ on a validated scale.

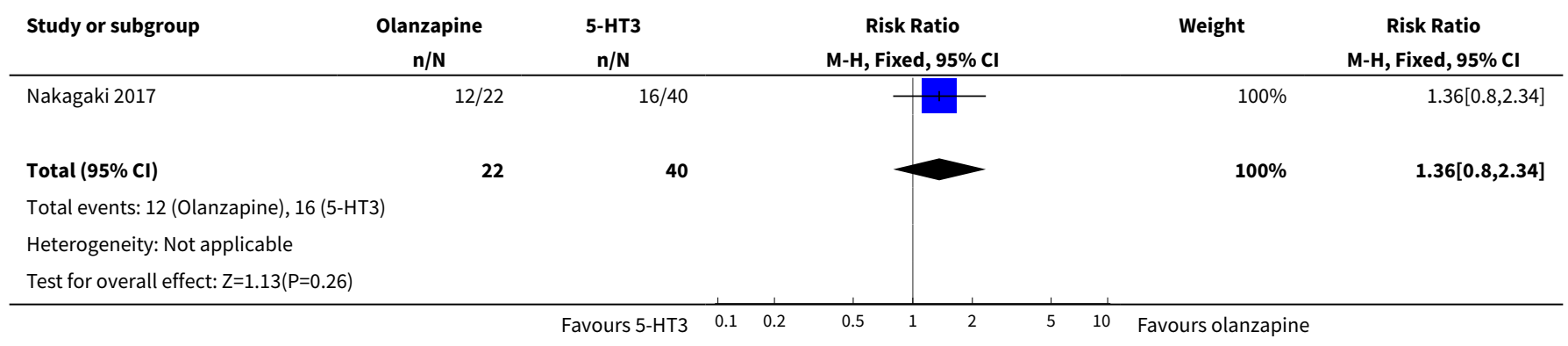

Analysis 4.3. Comparison 4 Olanzapine vs 5-HT3 antagonist, Outcome $350 \%$ improvement in nausea at $48 \mathrm{~h}$ on a validated scale.

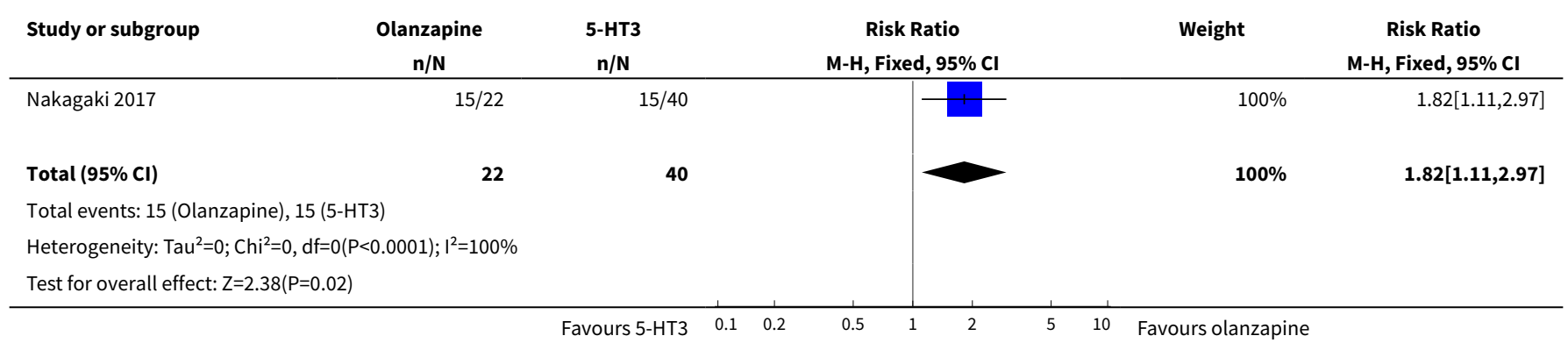

Analysis 4.4. Comparison 4 Olanzapine vs 5-HT3 antagonist, Outcome 4 Withdrawals due to all causes.

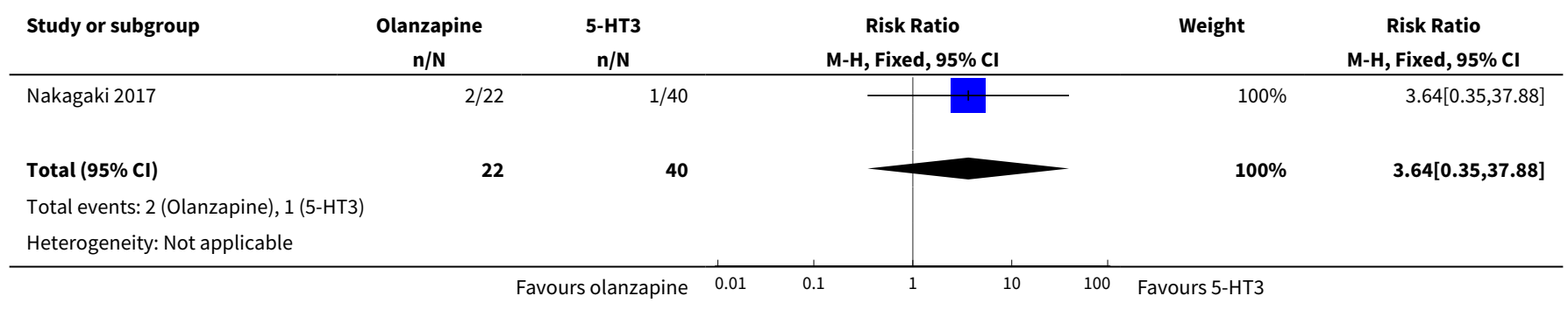




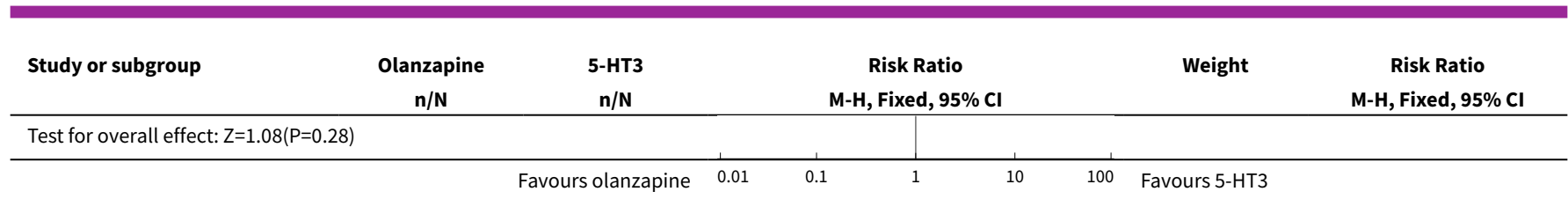

Analysis 4.5. Comparison 4 Olanzapine vs 5-HT3 antagonist, Outcome 5 Withdrawals due to lack of efficacy.

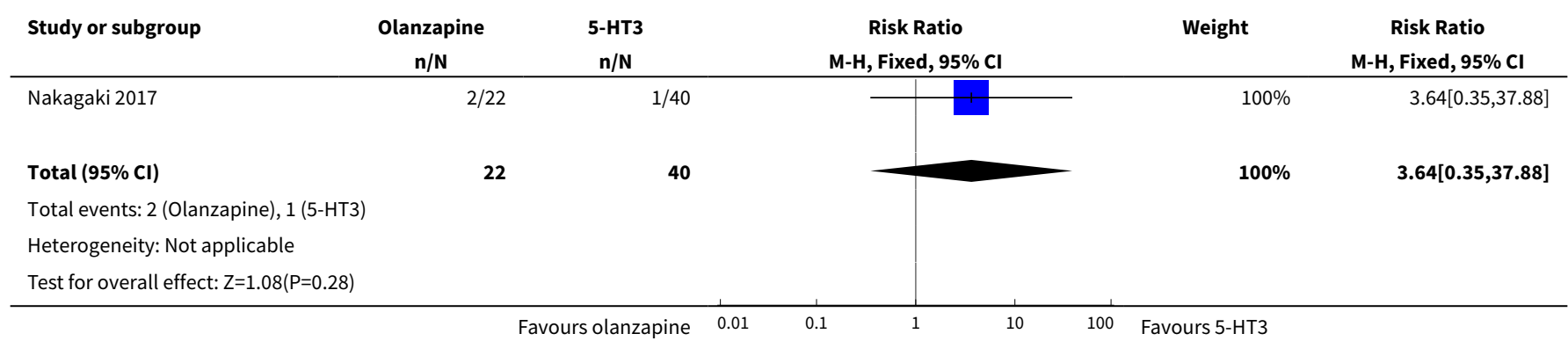

Analysis 4.6. Comparison 4 Olanzapine vs 5-HT3 antagonist, Outcome 6 Withdrawals due to adverse events.

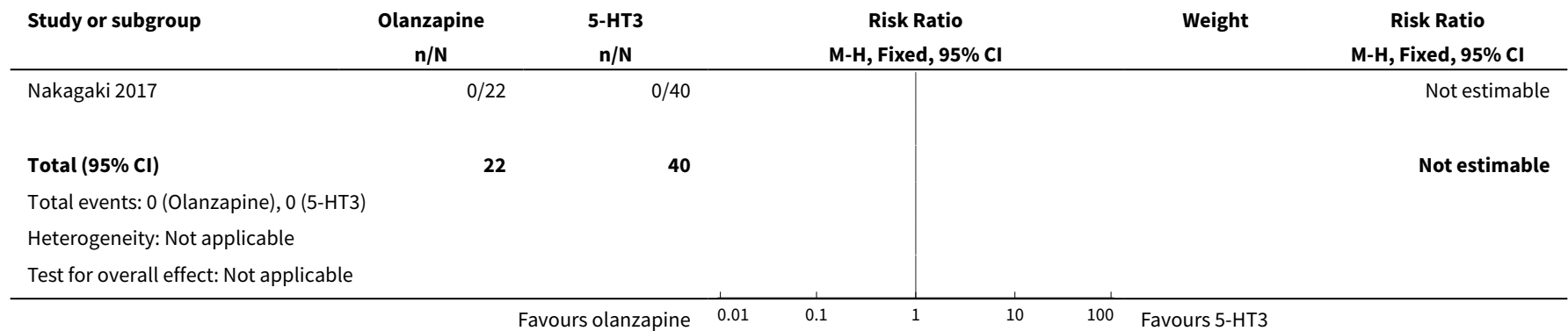

Analysis 4.7. Comparison 4 Olanzapine vs 5-HT3 antagonist, Outcome 7 Withdrawals due to death.

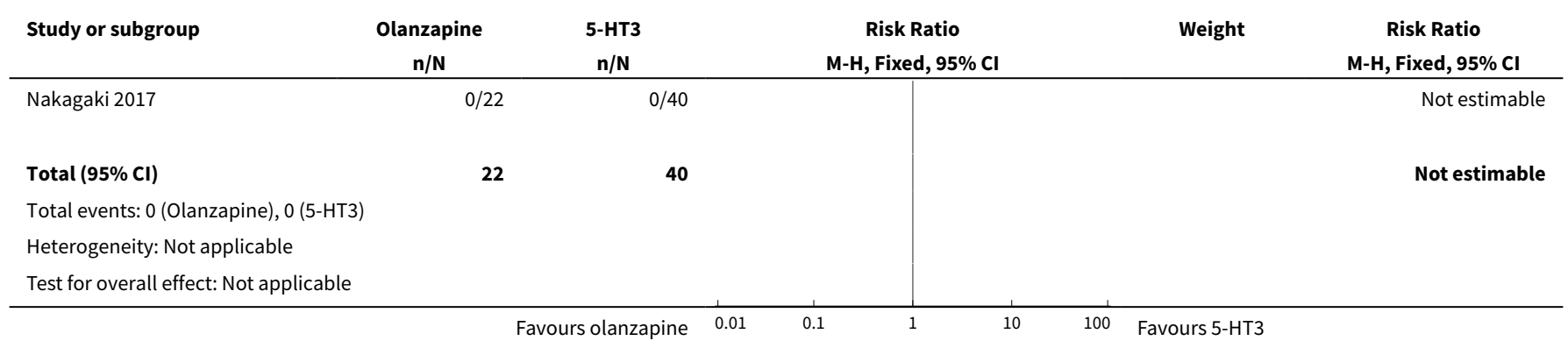


Comparison 5. Olanzapine vs dexamethasone

\begin{tabular}{|c|c|c|c|c|}
\hline Outcome or subgroup title & No. of studies & $\begin{array}{l}\text { No. of partici- } \\
\text { pants }\end{array}$ & Statistical method & Effect size \\
\hline 1 Serious adverse events & 1 & 229 & $\begin{array}{l}\text { Risk Ratio (M-H, Fixed, 95\% } \\
\mathrm{Cl})\end{array}$ & $0.0[0.0,0.0]$ \\
\hline 2 Withdrawals due to all causes & 1 & 229 & $\begin{array}{l}\text { Risk Ratio (M-H, Fixed, 95\% } \\
\mathrm{Cl})\end{array}$ & $0.0[0.0,0.0]$ \\
\hline 3 No nausea over trial period & 1 & 229 & $\begin{array}{l}\text { Risk Ratio (M-H, Fixed, 95\% } \\
\mathrm{Cl})\end{array}$ & $1.73[1.37,2.18]$ \\
\hline 4 No vomiting over trial period & 1 & 229 & $\begin{array}{l}\text { Risk Ratio (M-H, Fixed, 95\% } \\
\mathrm{Cl} \text { ) }\end{array}$ & $1.27[1.10,1.48]$ \\
\hline $\begin{array}{l}5 \text { No acute nausea (within } 24 \text { h of } \\
\text { chemotherapy) }\end{array}$ & 1 & 229 & $\begin{array}{l}\text { Risk Ratio (M-H, Fixed, 95\% } \\
\mathrm{Cl})\end{array}$ & $1.07[0.99,1.14]$ \\
\hline $\begin{array}{l}6 \text { No acute vomiting (within } 24 \text { h of } \\
\text { chemotherapy) }\end{array}$ & 1 & 229 & $\begin{array}{l}\text { Risk Ratio (M-H, Fixed, 95\% } \\
\mathrm{Cl})\end{array}$ & $1.01[0.94,1.08]$ \\
\hline $\begin{array}{l}7 \text { No delayed nausea ( } 1-5 \text { days after } \\
\text { chemotherapy) }\end{array}$ & 1 & 229 & $\begin{array}{l}\text { Risk Ratio (M-H, Fixed, 95\% } \\
\text { Cl) }\end{array}$ & $1.66[1.33,2.08]$ \\
\hline $\begin{array}{l}8 \text { No delayed vomiting ( } 1-5 \text { days after } \\
\text { chemotherapy) }\end{array}$ & 1 & 229 & $\begin{array}{l}\text { Risk Ratio (M-H, Fixed, 95\% } \\
\mathrm{Cl})\end{array}$ & $1.25[1.07,1.45]$ \\
\hline
\end{tabular}

Analysis 5.1. Comparison 5 Olanzapine vs dexamethasone, Outcome 1 Serious adverse events.

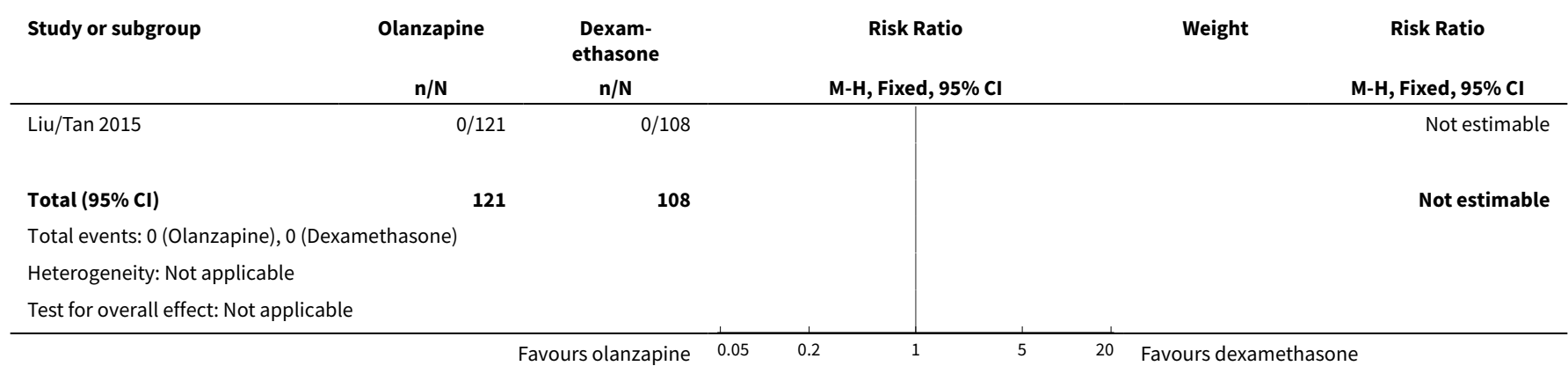

Analysis 5.2. Comparison 5 Olanzapine vs dexamethasone, Outcome 2 Withdrawals due to all causes.

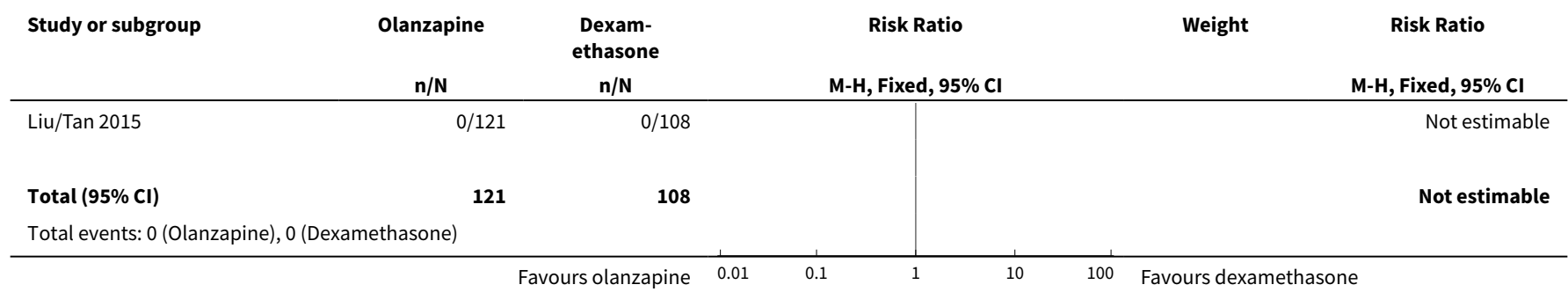




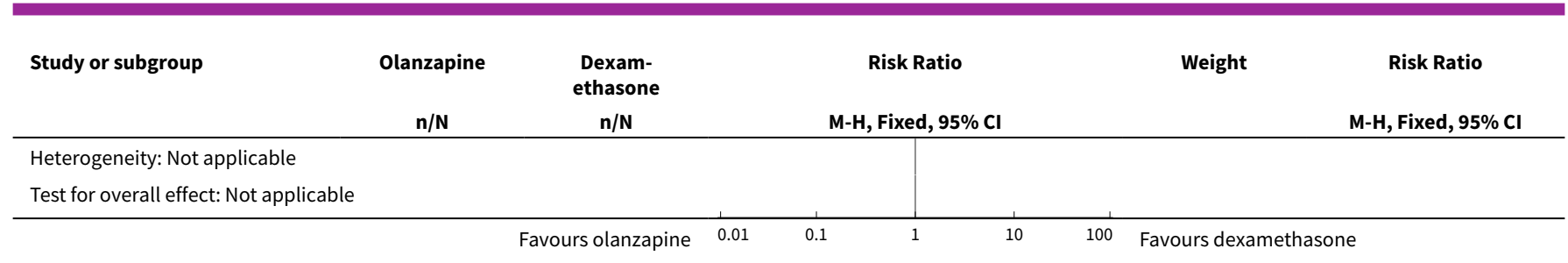

Analysis 5.3. Comparison 5 Olanzapine vs dexamethasone, Outcome 3 No nausea over trial period.

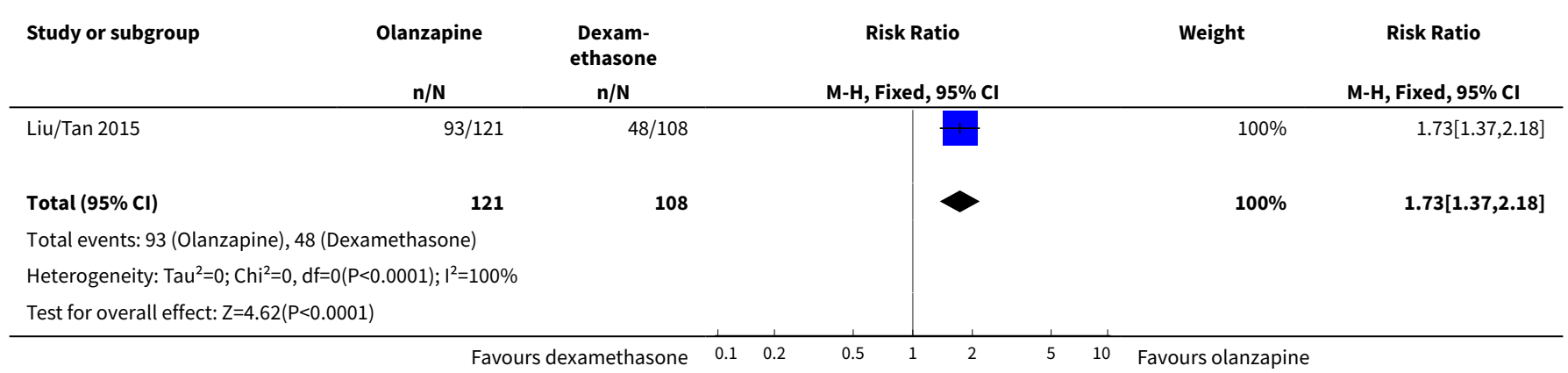

Analysis 5.4. Comparison 5 Olanzapine vs dexamethasone, Outcome 4 No vomiting over trial period.

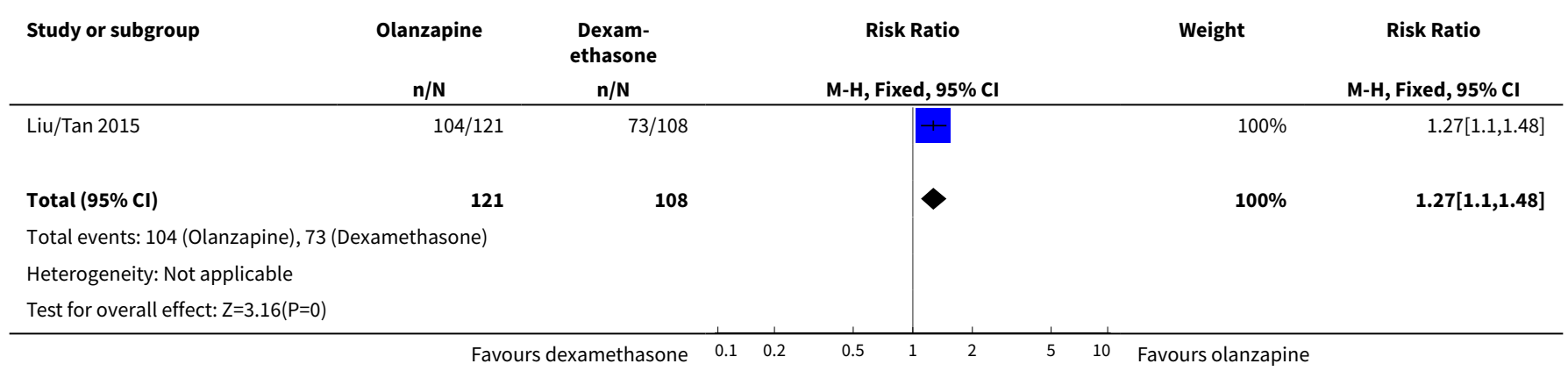

Analysis 5.5. Comparison 5 Olanzapine vs dexamethasone, Outcome 5 No acute nausea (within $24 \mathrm{~h}$ of chemotherapy).

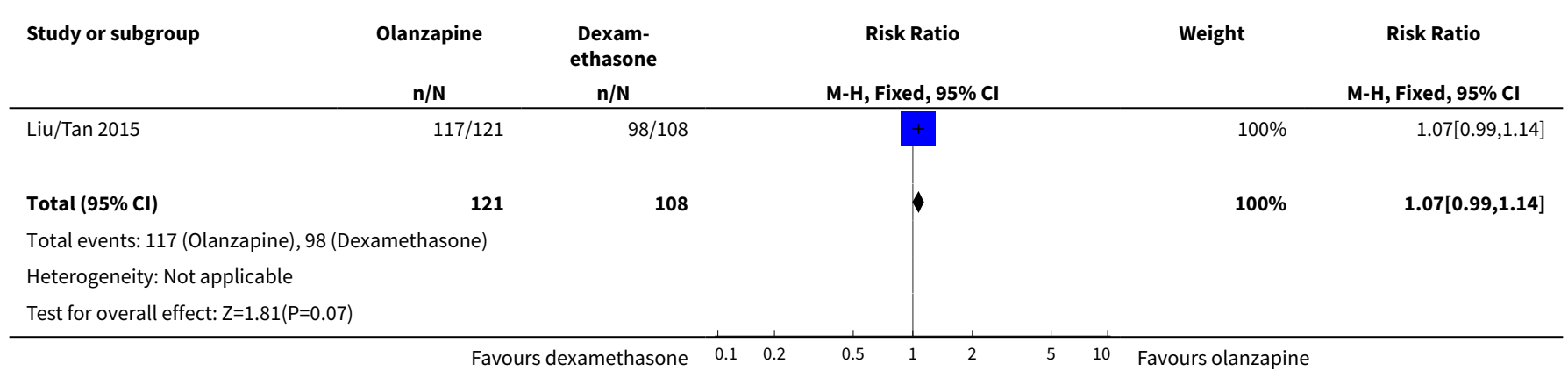


Analysis 5.6. Comparison 5 Olanzapine vs dexamethasone, Outcome 6 No acute vomiting (within 24 h of chemotherapy).

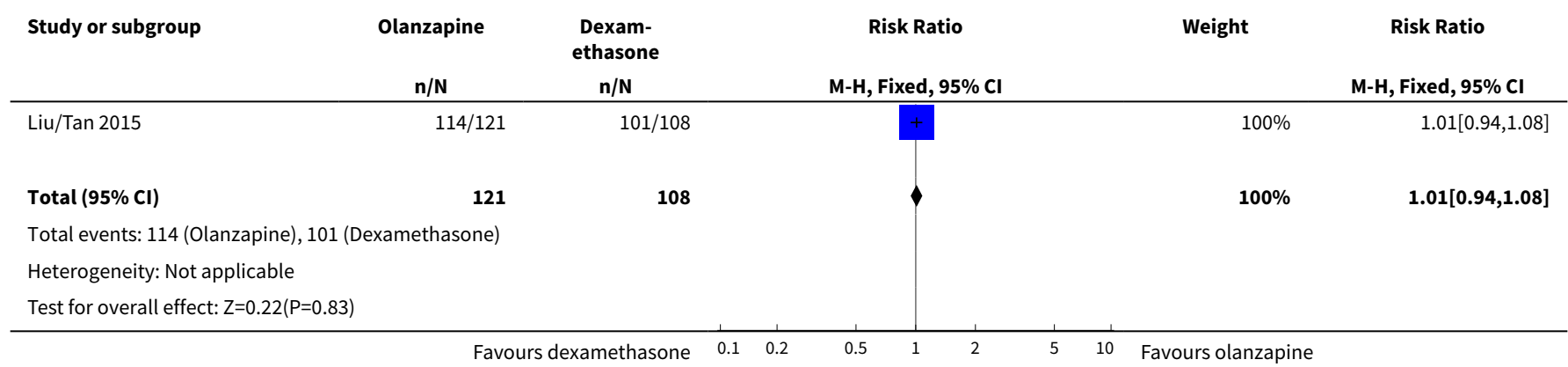

Analysis 5.7. Comparison 5 Olanzapine vs dexamethasone, Outcome 7 No delayed nausea (1-5 days after chemotherapy).

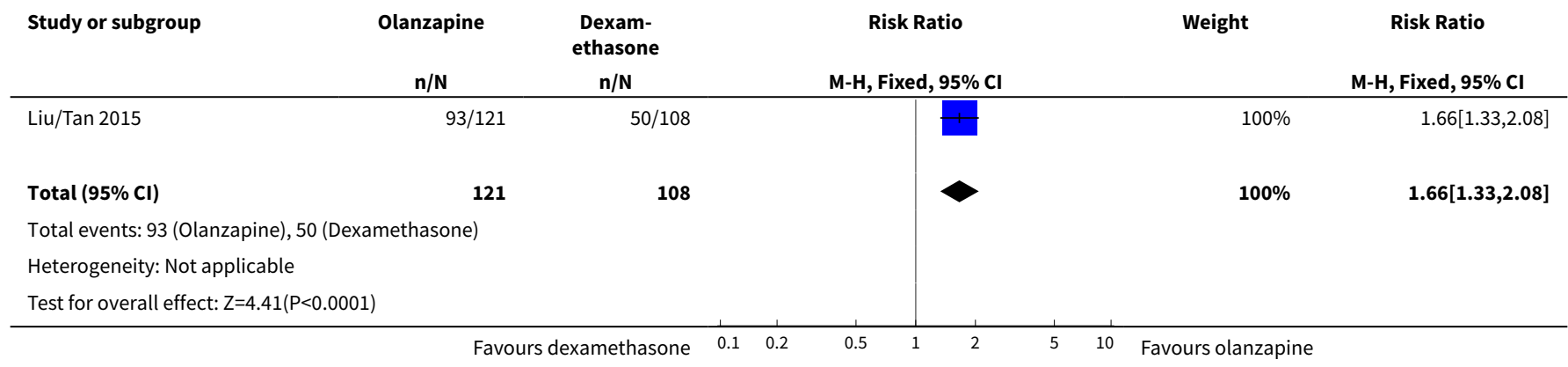

Analysis 5.8. Comparison 5 Olanzapine vs dexamethasone, Outcome 8 No delayed vomiting (1-5 days after chemotherapy).

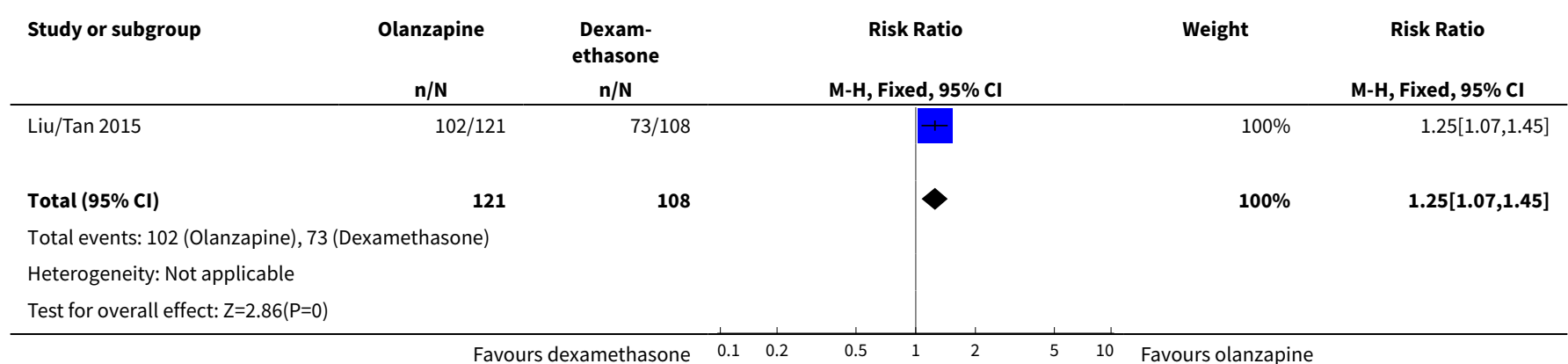


Comparison 6. Subgroup (dose): olanzapine vs placebo/no treatment

\begin{tabular}{|c|c|c|c|c|}
\hline $\begin{array}{l}\text { Outcome or sub- } \\
\text { group title }\end{array}$ & No. of studies & $\begin{array}{l}\text { No. of partici- } \\
\text { pants }\end{array}$ & Statistical method & Effect size \\
\hline $\begin{array}{l}1 \text { No nausea or vomit- } \\
\text { ing over trial period }\end{array}$ & 3 & 561 & Risk Ratio (M-H, Random, 95\% Cl) & $1.98[1.59,2.47]$ \\
\hline $1.15 \mathrm{mg}$ & 1 & 60 & Risk Ratio (M-H, Random, 95\% Cl) & $2.0[1.19,3.36]$ \\
\hline $1.210 \mathrm{mg}$ & 2 & 501 & Risk Ratio (M-H, Random, 95\% Cl) & $1.98[1.40,2.80]$ \\
\hline $\begin{array}{l}2 \text { Serious adverse } \\
\text { events }\end{array}$ & 7 & 889 & Risk Ratio (M-H, Fixed, 95\% Cl) & $2.46[0.48,12.55]$ \\
\hline $2.15 \mathrm{mg}$ & 5 & 388 & Risk Ratio (M-H, Fixed, 95\% Cl) & $0.0[0.0,0.0]$ \\
\hline $2.210 \mathrm{mg}$ & 2 & 501 & Risk Ratio (M-H, Fixed, 95\% Cl) & $2.46[0.48,12.55]$ \\
\hline $\begin{array}{l}3 \text { Other adverse } \\
\text { events }\end{array}$ & 4 & 332 & Risk Ratio (M-H, Fixed, 95\% Cl) & $1.71[0.99,2.96]$ \\
\hline $3.15 \mathrm{mg}$ & 2 & 128 & Risk Ratio (M-H, Fixed, 95\% Cl) & $4.0[0.46,34.85]$ \\
\hline $3.210 \mathrm{mg}$ & 2 & 204 & Risk Ratio (M-H, Fixed, 95\% Cl) & $1.55[0.88,2.73]$ \\
\hline 4 Somnolence/fatigue & 5 & 464 & Risk Ratio (M-H, Fixed, 95\% Cl) & $2.33[1.30,4.18]$ \\
\hline $4.15 \mathrm{mg}$ & 2 & 160 & Risk Ratio (M-H, Fixed, 95\% Cl) & $1.48[0.75,2.91]$ \\
\hline $4.210 \mathrm{mg}$ & 3 & 304 & Risk Ratio (M-H, Fixed, 95\% Cl) & $5.33[1.60,17.81]$ \\
\hline
\end{tabular}

Analysis 6.1. Comparison 6 Subgroup (dose): olanzapine vs placebo/ no treatment, Outcome 1 No nausea or vomiting over trial period.

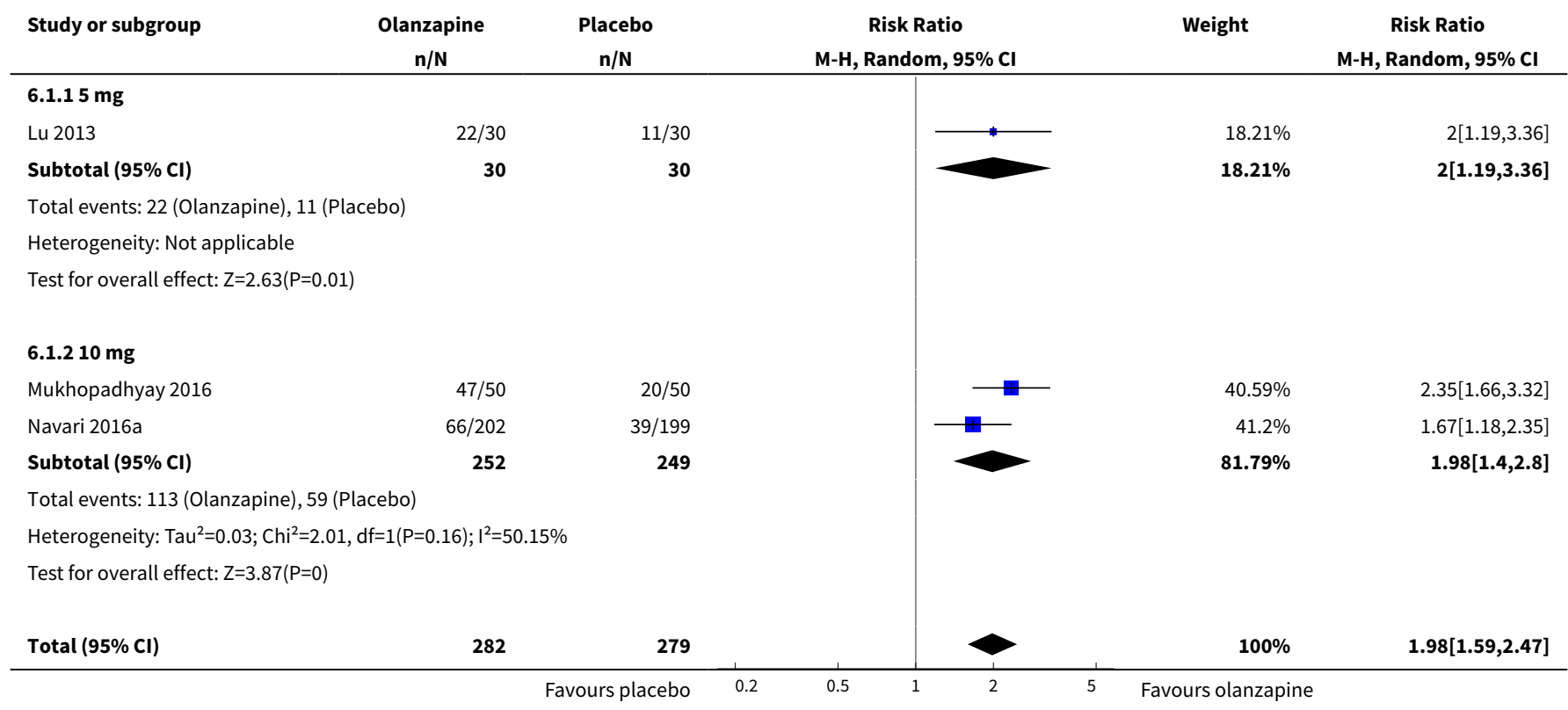




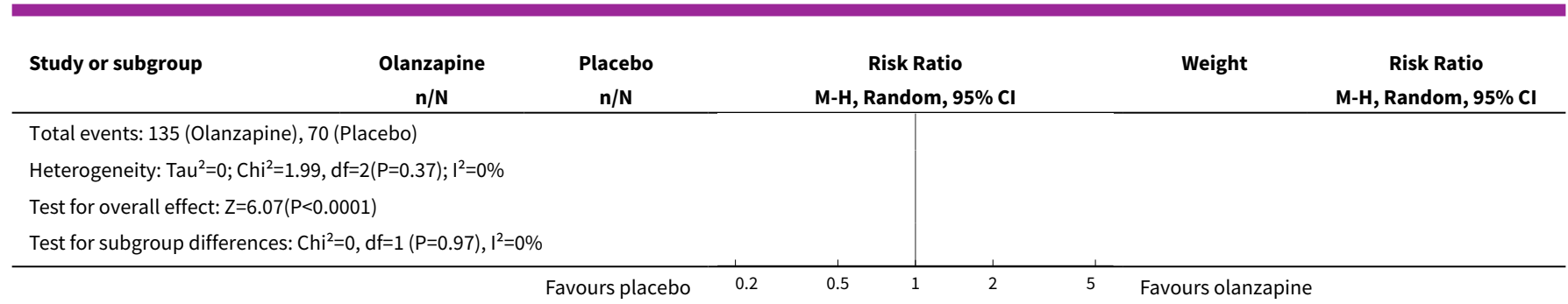

Analysis 6.2. Comparison 6 Subgroup (dose): olanzapine vs placebo/no treatment, Outcome 2 Serious adverse events.

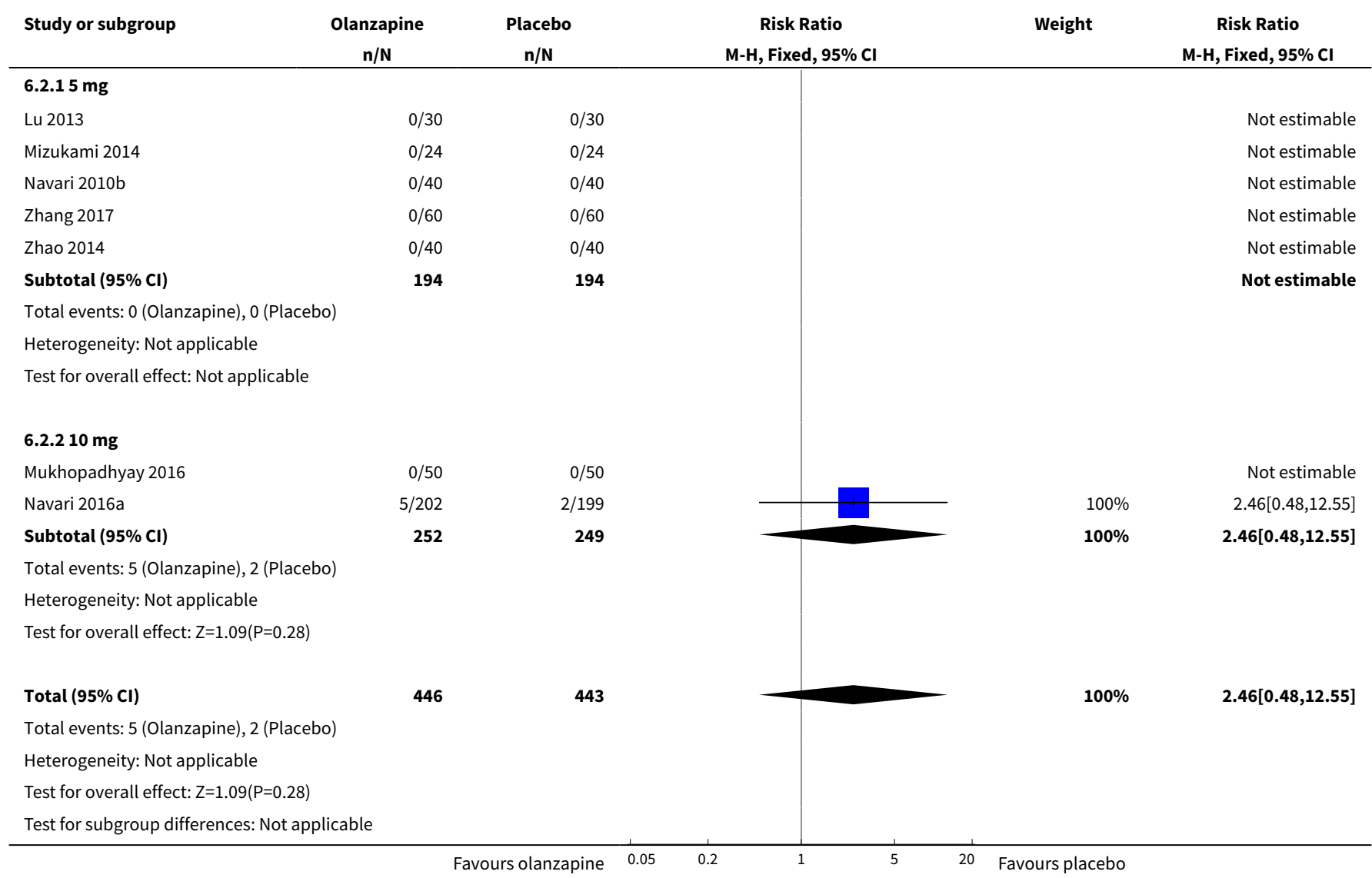

Analysis 6.3. Comparison 6 Subgroup (dose): olanzapine vs placebo/no treatment, Outcome 3 Other adverse events.

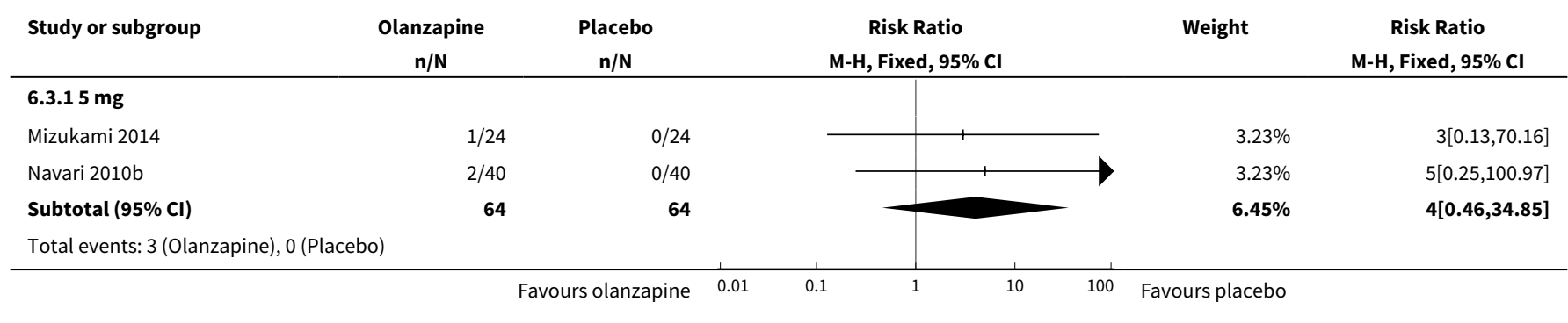




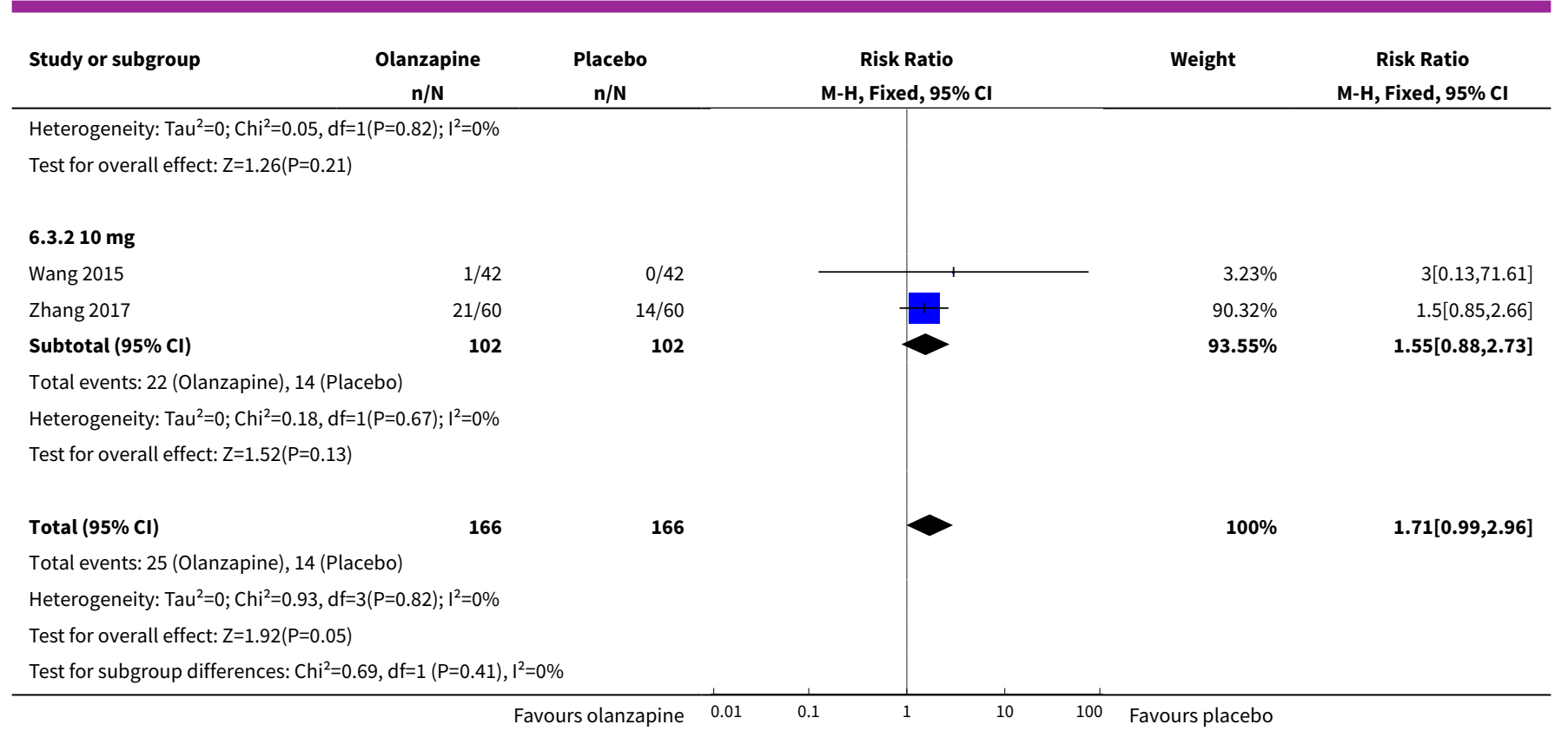

Analysis 6.4. Comparison 6 Subgroup (dose): olanzapine vs placebo/no treatment, Outcome 4 Somnolence/fatigue.

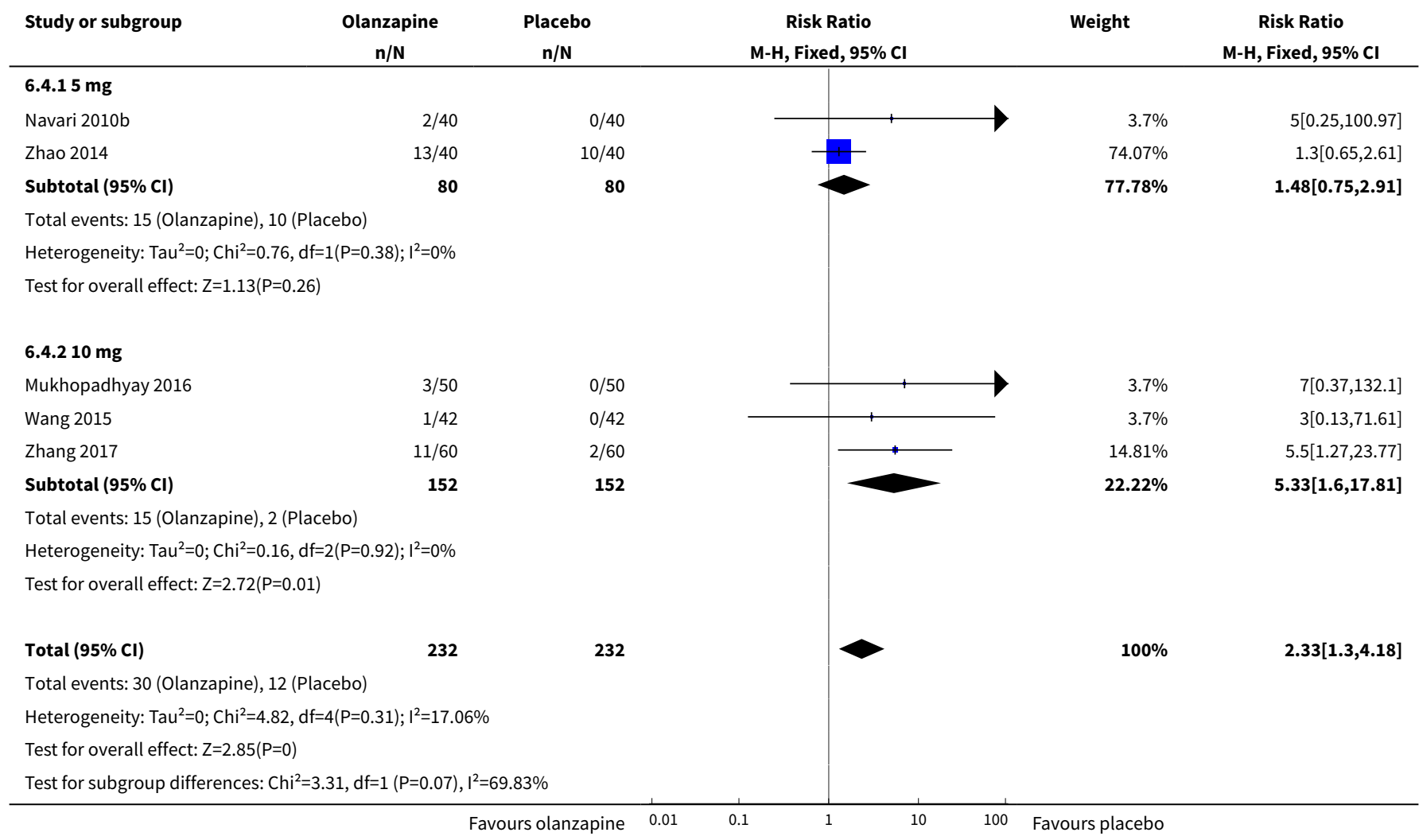




\section{APPE N D I CES}

\section{Appendix 1. MEDLINE search strategy}

1 Antipsychotic Agents/

2 (olanzapine or zyprexa).mp. [mp=title, abstract, original title, name of substance word, subject heading word, keyword heading word, protocol supplementary concept word, rare disease supplementary concept word, unique identifier, synonyms)

31 or 2

4 exp Neoplasms/

5 (cancer ${ }^{\star}$ or neoplas* or tumo* or carcinoma* or hodgkin* or nonhodgkin* or adenocarcinoma* or leuk? emia* or metasta* or malignan ${ }^{\star}$ or lymphoma* or sarcoma* or melanoma* or myeloma* or oncolog* or chemotherapy $\left.{ }^{\star}\right)$.tw.

6 Nausea/

7 vomiting/ or vomiting, anticipatory/

8 (nause* or vomit* or sick or retch ${ }^{\star}$ or emetic ${ }^{\star}$ or emesis).tw.

9 Antiemetics/

10 anti $^{*}$ eme ${ }^{\star}$. tw.

116 or 7 or 8 or 9 or 10

124 or 5

133 and 11 and 12

\section{Appendix 2. CENTRAL (CRSO) search strategy}

\#1 MESH DESCRIPTOR Antipsychotic Agents

\#2 ((olanzapine or zyprexa)):TI,AB,KY

\#3 \#1 OR \#2

\#4 MESH DESCRIPTOR Neoplasms EXPLODE ALL TREES

\#5 ((cancer* or neoplas* or tumo* or carcinoma* or hodgkin* or nonhodgkin* or adenocarcinoma* or leuk?emia* or metasta* or malignan* or lymphoma* or sarcoma* or melanoma* or myeloma* or oncolog* or chemotherap $\left.\left.{ }^{\star}\right)\right): \mathrm{TI}, \mathrm{AB}, \mathrm{KY}$

\#6 \#4 OR \#5

\#7 MESH DESCRIPTOR Nausea

\#8 MESH DESCRIPTOR vomiting

\#9 MESH DESCRIPTOR vomiting, anticipatory

$\# 10$ (nause* or vomit* $^{\star}$ or sick or retch* or emetic ${ }^{\star}$ or emesis)

\#11 MESH DESCRIPTOR Antiemetics

$\# 12$ anti $^{\star} e m e^{\star}: \mathrm{TI}, \mathrm{AB}, \mathrm{KY}$

\#13 \#7 OR \#8 OR \#9 OR \#10 OR \#11 OR \#12

\#14 \#3 AND \#6 AND \#13

\section{Appendix 3. Embase (OVID) search strategy}

1 Neuroleptic Agent/

2 (olanzapine or zyprexa).mp. [mp=title, abstract, heading word, drug trade name, original title, device manufacturer, drug manufacturer, device trade name, keyword, floating subheading) 
31 or 2

4 exp Neoplasm/

5 (cancer* or neoplas* or tumo* or carcinoma* or hodgkin* or nonhodgkin* or adenocarcinoma* or leuk? emia* or metasta* or malignan* or lymphoma* or sarcoma* or melanoma* or myeloma* or oncolog* or chemotherapy*).tw.

6 "Nausea and vomiting"/

7 ((anticipatory nausea.mp. and vomiting/) or chemotherapy induced nausea.mp.) and vomiting/ [mp=title, abstract, heading word, drug trade name, original title, device manufacturer, drug manufacturer, device trade name, keyword, floating subheading)

8 (nause $^{\star}$ or vomit ${ }^{\star}$ or sick or retch ${ }^{\star}$ or emetic ${ }^{\star}$ or emesis).tw.

9 Antiemetic agent/

10 anti $^{\star}$ eme ${ }^{\star}$. tw.

116 or 7 or 8 or 9 or 10

124 or 5

133 and 11 and 12

\section{Appendix 4. Published search overview}

\begin{tabular}{llll}
\hline Database searched & $\begin{array}{l}\text { Number of results } \\
\text { February 2017 }\end{array}$ & $\begin{array}{l}\text { Number of results } \\
\text { 6 September 2017 }\end{array}$ & $\begin{array}{l}\text { Number of results } \\
\mathbf{2 0} \text { September } \\
\mathbf{2 0 1 7}\end{array}$ \\
\hline CENTRAL (CRSO) & 36 & 9 & 62 \\
\hline $\begin{array}{l}\text { MEDLINE \& MEDLINE in Process (OVID) 1946 to 5 September } \\
2017\end{array}$ & 110 & 2 & 190 \\
\hline Embase (OVID) 1974 to 2017 week 36 & 251 & 22 & 695 \\
\hline Total & $\mathbf{3 9 7}$ & $\mathbf{3 3}$ & $\mathbf{9 4 7}$ \\
\hline After de-duplication & $\mathbf{3 4 9}$ & $\mathbf{2 1}$ & $\mathbf{7 2 5}$ \\
\hline
\end{tabular}

\section{Appendix 5. Unpublished search overview}

\begin{tabular}{lll}
\hline Unpublished databases searched & Date last searched & $\begin{array}{l}\text { Number of results September } \\
\mathbf{2 0 1 7}\end{array}$ \\
\hline clinicaltrials.gov & 8 September 2017 & 20 trials \\
\hline apps.who.int/trialsearch & 8 September 2017 & 17 trials \\
\hline Total & & $\mathbf{3 7}$ \\
\hline After de-duplication & $\mathbf{2 4}$ \\
\hline
\end{tabular}




\begin{tabular}{lll}
\hline Results & $\begin{array}{l}\text { Number of results } \\
\text { September 2017 }\end{array}$ & $\begin{array}{c}\text { Number already pub- } \\
\text { lished study }\end{array}$ \\
\hline Completed trials meeting inclusion criteria with results & 3 & 1 \\
\hline Completed trials meeting inclusion criteria without results & 1 & - \\
\hline Ongoing trials potentially meeting inclusion criteria & 9 & - \\
\hline Terminated trials & 2 & N/A \\
\hline Excluded trials & 11 & \\
\hline
\end{tabular}

\section{CONTRIBUTIONS OF AUTHORS}

AS wrote the protocol, with input from the other authors and Phil Wiffen.

$\mathrm{AS}, \mathrm{KN}$ and $\mathrm{KH}$ selected studies for inclusion.

AS retrieved the full texts.

AS, KH, LW and EP extracted data.

AS and $\mathrm{KH}$ entered data into Review Manager 5 and carried out the analyses. Review authors reached unanimous decisions on the comparison pairs and changes to protocol.

AS interpreted the results and wrote the full review with input from all authors, particularly in relation to interpretation of the analysis, editing and finalising the full review.

All authors will be responsible for updates.

\section{DECLARATIONS OF INTEREST}

- AS: none known. AS is a specialist trainee Palliative Medicine physician and manages patients with advanced life-threatening illnesses, including cancer.

- $\mathrm{KN}$ : none known. $\mathrm{KN}$ is a specialist Palliative Medicine Consultant physician and manages patients with advanced life-threatening illnesses, including cancer.

- EP: none known.

- KH: none known.

- LW: none known. LW is a retired GP and Senior Cochrane UK fellow.

- MB: none known. MB is a specialist Ear, Nose and Throat Consultant surgeon and manages patients with illnesses affecting the ear, nose and throat, including cancer. Professor MB is joint Co-ordinating Editor of Cochrane ENT, but had no role in the editorial process for this review.

- BW: none known. BW is a specialist Palliative Medicine Consultant physician and manages patients with advanced life-threatening illnesses, including cancer. BW is also National Clinical Director for End of Life Care but she has no involvement in commissioning pharmaceutical agents.

\section{SOURCES OF SUPPORT}

\section{Internal sources}

- Cochrane UK Fellowship, UK.

AS received funding from Health Education Thames Valley in order to undertake this systematic review as part of the Cochrane UK Fellowship.

\section{External sources}

- No sources of support supplied 


\section{DIFFERENCES BETWEEN PROTOCOLAND REVIEW}

We noted that somnolence and fatigue were the most frequently occurring of the 'other adverse events'. We therefore reported these separately in the "Methods" and "Results" sections of the quantitative analysis and in the 'Summary of findings' tables, in addition to the 'other adverse events' reporting.

In the results and 'Summary of findings' tables we have reported both odds ratio and risk ratio, as we acknowledge that in our data set risk ratio appears to underestimate the $\mathrm{I}^{2}$ result.

\section{N D EX TERMS}

\section{Medical Subject Headings (MeSH)}

Antiemetics [adverse effects] [*therapeutic use]; Antineoplastic Agents [adverse effects]; Benzodiazepines [adverse effects] [*therapeutic use]; Chemoradiotherapy [adverse effects]; Dexamethasone [therapeutic use]; Disorders of Excessive Somnolence [chemically induced]; Fatigue; Metoclopramide [therapeutic use]; Nausea [ ${ }^{*}$ drug therapy] [etiology] [prevention \& control]; Neoplasms [ ${ }^{*}$ complications]; Olanzapine; Quality of Life; Randomized Controlled Trials as Topic; Vomiting [*drug therapy] [etiology] [prevention \& control]

\section{MeSH check words}

Adult; Humans 